\title{
Towards uncovering polyomavirus-carrying human cancers
}

Citation for published version (APA):

Klufah, F. M. (2022). Towards uncovering polyomavirus-carrying human cancers: pathology and implications for tumorigenesis. [Doctoral Thesis, Maastricht University]. ProefschriftMaken. https://doi.org/10.26481/dis.20220119fk

Document status and date:

Published: 01/01/2022

DOI:

10.26481/dis.20220119fk

Document Version:

Publisher's PDF, also known as Version of record

\section{Please check the document version of this publication:}

- A submitted manuscript is the version of the article upon submission and before peer-review. There can be important differences between the submitted version and the official published version of record.

People interested in the research are advised to contact the author for the final version of the publication, or visit the DOI to the publisher's website.

- The final author version and the galley proof are versions of the publication after peer review.

- The final published version features the final layout of the paper including the volume, issue and page numbers.

Link to publication

\footnotetext{
General rights rights.

- You may freely distribute the URL identifying the publication in the public portal. please follow below link for the End User Agreement:

www.umlib.nl/taverne-license

Take down policy

If you believe that this document breaches copyright please contact us at:

repository@maastrichtuniversity.nl

providing details and we will investigate your claim.
}

Copyright and moral rights for the publications made accessible in the public portal are retained by the authors and/or other copyright owners and it is a condition of accessing publications that users recognise and abide by the legal requirements associated with these

- Users may download and print one copy of any publication from the public portal for the purpose of private study or research.

- You may not further distribute the material or use it for any profit-making activity or commercial gain

If the publication is distributed under the terms of Article $25 \mathrm{fa}$ of the Dutch Copyright Act, indicated by the "Taverne" license above, 
Towards Uncovering Polyomavirus-Carrying Human Cancers:

Pathology and Implications for Tumorigenesis

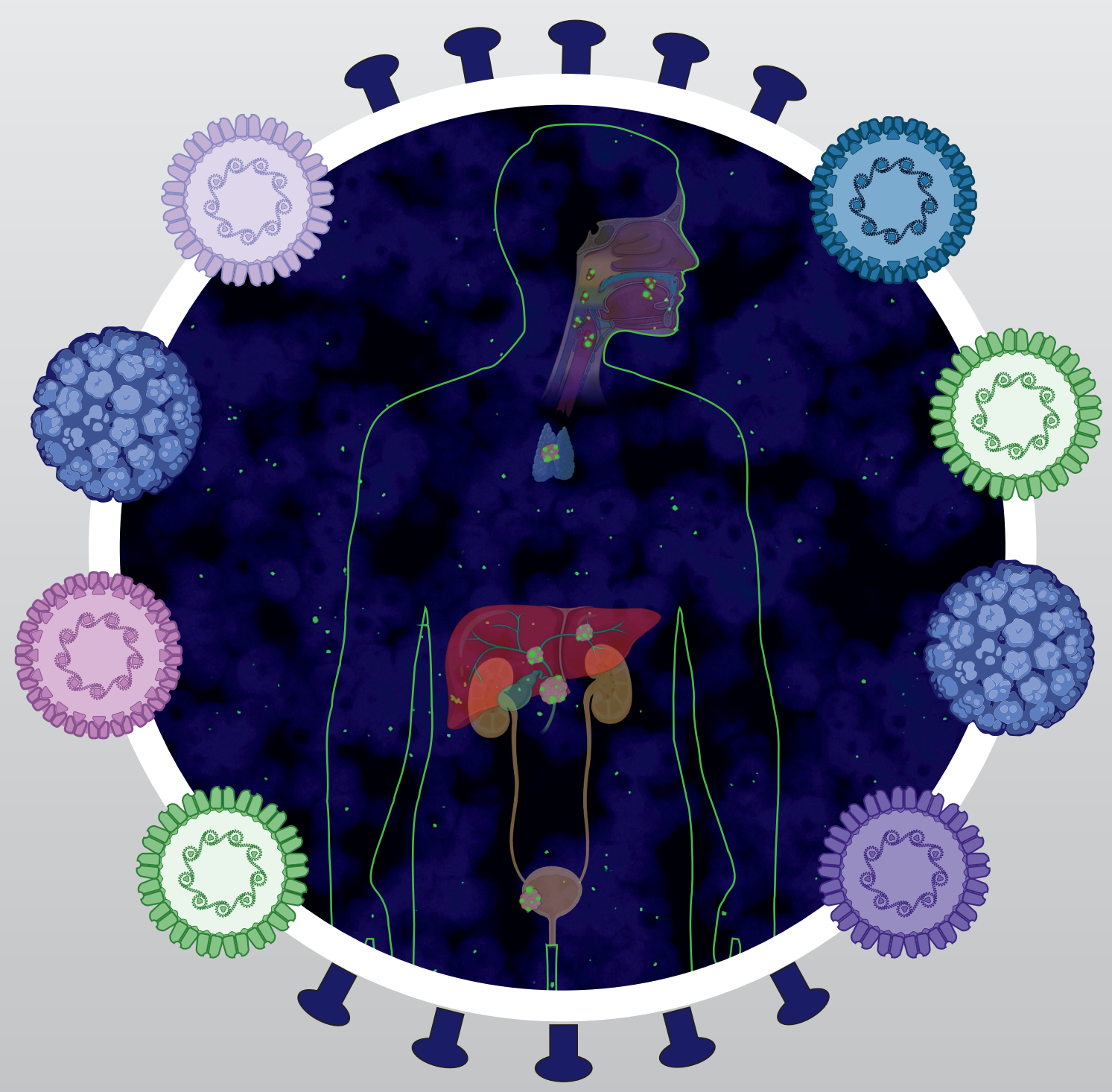

Faisal Mahdi Klufah 



\section{Towards Uncovering Polyomavirus-Carrying Human Cancers: \\ Pathology and Implications for Tumorigenesis}

Faisal Mahdi Klufah 
The studies in this thesis were performed in the Pathology Department, Maastricht University Medical Centre+ within the School for Oncology and Developmental Biology (GROW) at the faculty of Health, Medicine, and life sciences at Maastricht University. This thesis was financially supported by Albaha University, Saudi Arabia.

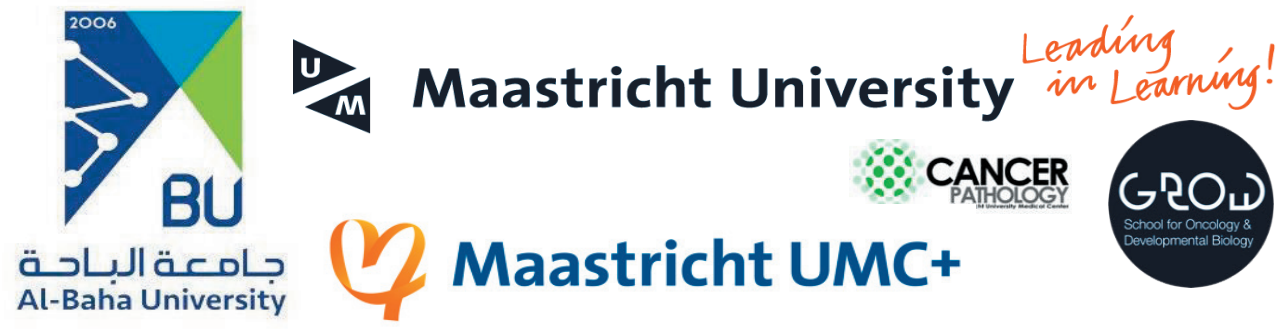

ISBN: 978-94-6423-597-5

Printing: ProefschriftMaken || www.proefschriftmaken.nl

(C) Copyright Faisal Mahdi Klufah, 2021

All rights reserved. No part of this publication may be reproduced, stored in a retrieval system, or transmitted in any form or by any means, electronic, mechanical, photocopying, recording or otherwise, without prior written permission from the author, or when applicable, from the copyright-owning journals for previous published chapters. 


\title{
Towards Uncovering Polyomavirus-Carrying Human Cancers: \\ Pathology and Implications for Tumorigenesis
}

\author{
DISSERTATION
}

To obtain the degree of Doctor at Maastricht University, on the authority of the Rector Magnificus,

Prof.dr. Rianne M. Letschert

in accordance with the decision of the Board of Deans,

to be defended in public on Wednesday $19^{\text {th }}$ of January 2022, at 10.00 hours

By

Faisal Mahdi Klufah

Born 26 $6^{\text {th }}$ October 1984 in Jazan, Saudi Arabia 


\section{Promotores:}

Prof. Dr. Axel zur Hausen

Prof. Dr. Ernst-Jan Speel

\section{Co-promotor:}

Dr. Véronique Winnepenninckx

Assessment Committee:

Prof. Dr. M.G.J Tilanus (Chairman)

Prof. Dr. Ugo Lionel Moens (The Arctic University of Norway)

Prof. Dr. GA van Koeveringe

Dr. A.J.C van den Brule (Jeroen Bosch Hospital, 's-Hertogenbosch, The Netherlands) 


\section{Table of Contents}

CHAPTER 1 General introduction

CHAPTER 2 Emerging Role of Human Polyomaviruses 6 and 7 in

Human Cancers

CHAPTER 3 High Prevalence of Human Polyomavirus 7 in

Cholangiocarcinomas and Adjacent Peritumoral

Hepatocytes: Preliminary Findings

CHAPTER 4 Reactivation of BK Polyomavirus in Urine Cytology is

Not Associated with Urothelial Cell Carcinoma

CHAPTER 5 Low Prevalence of Merkel Cell Polyomavirus in Human

Epithelial Thymic Tumors

CHAPTER 6 Presence of Human Papillomavirus and Epstein-Barr

Virus, but Absence of Merkel Cell Polyomavirus, in

Head and Neck Cancer of Non-Smokers and Non-Drinkers 123

CHAPTER 7 General discussion

$\begin{array}{lll}\text { ADDENDUM Summary } & 165\end{array}$

Impact

171

List of abbreviations

181

Acknowledgments

Curriculum Vitae

List of publications and congresses participation

Summary in Arabic

203 


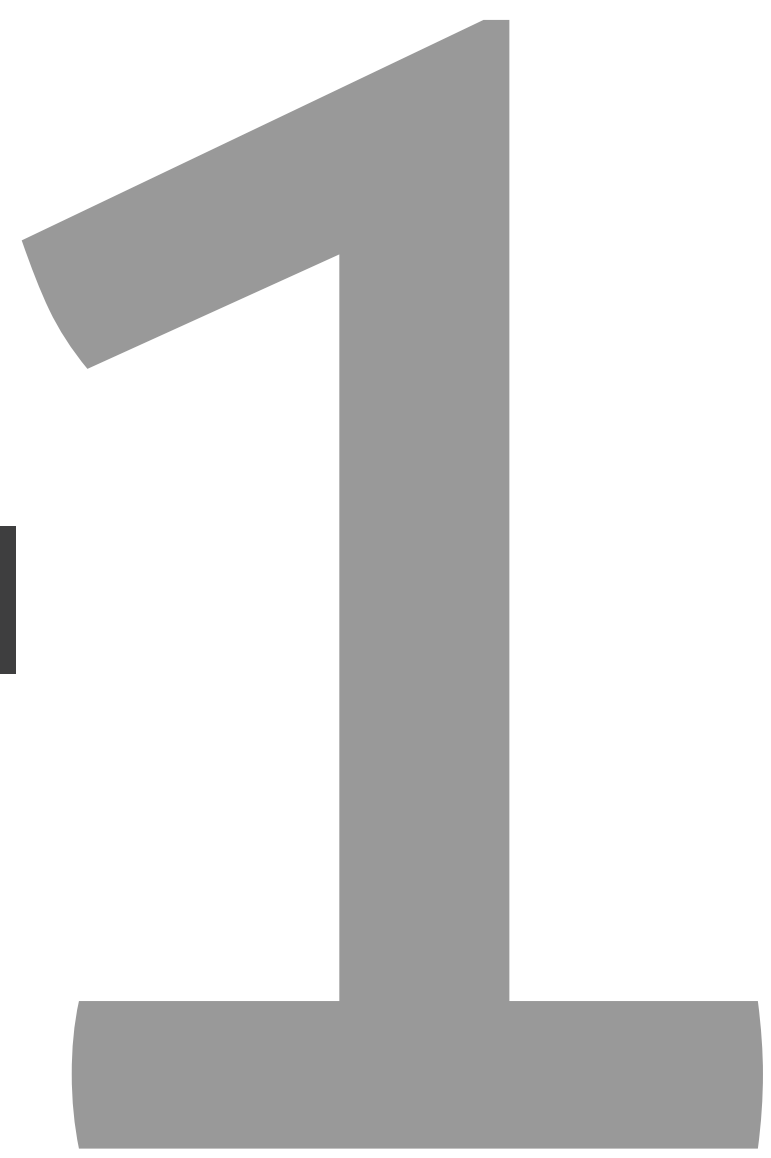


General introduction 


\section{General Introduction}

Cancer is one of the leading causes of death in humans worldwide and cancer-related mortality is still globally increasing ${ }^{1,2}$. In 2018 , approximately 18 million new cancer cases have been diagnosed, and cancer accounted for 9.6 million deaths in that same year worldwide ${ }^{1}$. Of interest, trends in incidence and mortality rates of tumors reveal wide geographical variations ${ }^{1,3}$. The most common risk factors for cancer include exposure to chemicals, smoking, alcohol, radiation, and inherited genetic mutations ${ }^{1,2}$. Amongst many other factors, infectious agents play an essential role in the etiology of cancer ${ }^{1,2}$. Infections constitute a well-established causative link with an increased cancer risk in humans, and it is estimated that up to $20 \%$ of cancers worldwide are associated with highrisk infectious agents ${ }^{2,4-6}$. However, the etiology of most human cancers is still unknown. Therefore, screening for emerging infectious agents potentially causing or contributing to human tumorigenesis is crucial to effectively avoid and limit exposure to these potential carcinogenic agents. Ultimately, identifying the presence of novel human tumor viruses or other infectious agents in cells and tissues may be an important opportunity for cancer prevention and early detection if studies will prove their causal/functional contribution to the development of pre-malignant lesions and progression to cancer.

\section{Infectious agents}

Several lines of evidence have established an association between infectious agents and tumorigenesis. According to the GLOBOCAN data in 2018, nearly 2.2 million cancer cases were related to specific infections ${ }^{4}$. Based on the International Agency for Research on Cancer (IARC) report, eight viruses, three parasites, and one bacterium infectious agent have been classified as carcinogens ${ }^{2,7,8}$. Helicobacter pylori is a gram-negative bacterium causing non-cardia gastric cancer and low-grade B-cell MALT lymphoma ${ }^{9}$. Three macroparasitic infections have been associated with the etiopathogenesis of human cancers, of which Opisthorchis viverrine and Clonorchis sinensis were found to be associated with cholangiocarcinoma, while Schistosoma haematobium infection is the one associated with inducing bladder carcinoma ${ }^{6,7}$.

Oncogenic viruses play a major role in human cancers and currently eight human viruses have been shown to be oncogenic in humans. Five of these oncogenic viruses are well-known DNA viruses, i.e Epstein-Barr virus (EBV), hepatitis B virus (HBV), human papillomaviruses (HPVs), Kaposi's sarcoma herpesvirus (KSHV), and Merkel cell polyomavirus $(\mathrm{MCPyV})^{2,7,8}$. The remaining three are RNA viruses, i.e. the human T-lymphotropic virus-1 (HTVL-1), hepatitis C virus (HCV), and the human immunodeficiency virus type 1 (HIV-1) ${ }^{6-8,10,11}$. A more detailed list of these human tumor viruses and the related human cancers is shown in Table1. 


\section{Viruses and tumors}

The significance of tumor viruses in human carcinogenesis has been studied intensely, and the discovery and characterization of oncogenic viruses paved the way to understand the etiopathogenesis, diagnosis, and treatment of a variety of human malignancies ${ }^{2}$. It is of particular interest to both identify and study potential human tumor viruses to develop potential vaccines, treatments, and prevention plans. It is generally accepted that infection with a potential human tumor-virus alone is not enough to cause human cancers. Additional predisposing factors appear to be involved, such as immunosuppression or environmental factors.

\section{Viruses as direct or indirect carcinogens}

Oncogenic viruses exert their oncogenic potential either by direct or indirect contribution to carcinogenesis Figure $1^{12,13}$. To date, five human oncogenic DNA-viruses have been identified, four of which are directly involved in the tumorigenesis, e.g. by the expression of viral oncoproteins that deregulate cellular tumor suppressor pathways ${ }^{2,8,12,13}$. A defined set of viral proteins of EBV plays a direct role in the cellular transformation to Burkitt's lymphoma through targeting cellular suppressor genes ${ }^{14,15}$. The direct role of HPV replication in oncogenesis is well established by the integration of the viral genome into the cell, and the viral early genes E6 and E7 have the oncogenic property to transform cells ${ }^{16-20}$. The closely related KSHV genome encodes oncogenic proteins to target cellular tumor suppressor proteins that may induce Kaposi sarcoma (KS) ${ }^{21,22}$. MCPyV is the most recently identified DNA-tumor virus, which was found to be clonally integrated into the Merkel cell carcinoma (MCC) genome and carries tumor-specific mutations within the large tumor antigen (LTAg) ${ }^{10,11,23}$. HBV is the only known DNA-virus that is indirectly promoting the development of hepatocellular carcinoma (HCC) through induction of chronic inflammation ${ }^{24-27}$.

Next to these DNA-tumor viruses, three RNA viruses have been identified to be associated with human carcinogenesis. HTVL-1 is a retrovirus linked directly to the tumorigenesis of aggressive T-cell lymphoma (ATL) ${ }^{28}$. Tax is the known HTVL-1 oncoprotein that plays key role in the transformation of human T-lymphocytes to ATL ${ }^{29}$. HCV and HIV-1 are the other two RNA-tumor viruses that induce human cancer by indirect mechanisms via the interaction between viral proteins, environmental factors, inflammation and/or hereditary risk factors Figure $1^{12,25,30-34}$.

Viral infections and subsequent viral latency precede the development of human cancers years before clinical detection. Latent infections cause chronic inflammatory reactions due to the immune response to chronically persisting exogenous viral antigens, which are not sufficiently eradicated by the immune system and thus results via acute inflammation in chronic inflammation ${ }^{35,36}$. Reactive oxygen radicals can be produced years later 


\section{Direct Viral Carcinogenesis}

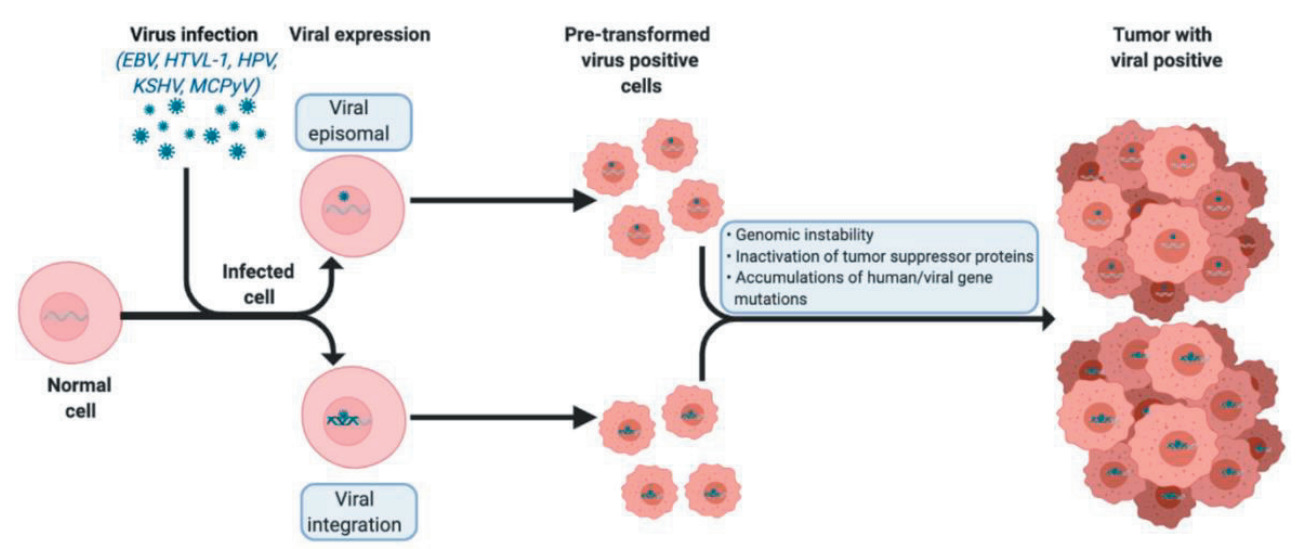

\section{Indirect Viral Carcinogenesis}

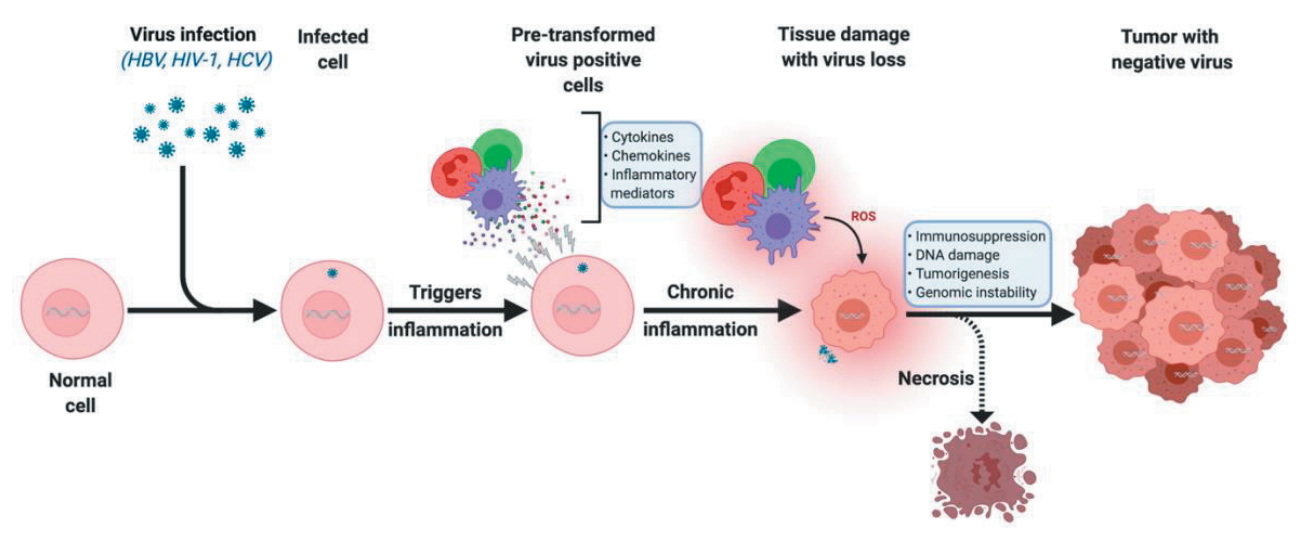

Figure 1: Models of direct and indirect viral carcionogenesis in humans. ROS; reactive oxygen species. (created in BioRender.com)

following chronic inflammation and may trigger modifications in the host nucleic acids and subsequently induce transformation, as it is known for $\mathrm{HBV}$ and $\mathrm{HCV}$ viruses ${ }^{35}$, ${ }^{37}$. Persistent infections with HBV or HCV viruses are responsible for most HCC cases in $\mathrm{HBV}$ and $\mathrm{HCV}$ endemic regions due to the indirect effects of chronic inflammation and progressive fibrosis ${ }^{24-26,38}$. For instance, $\mathrm{HCV}$ virus is not detected in the cancer cells that may develop after many years of infection but is often found in the lesions adjacent to the hepatocellular carcinoma ${ }^{30-33}$. Some other tumor viruses are incapable of transforming cells by itself, and rather serve as a cofactor for some malignancies, such as postulated for HIV-1 ${ }^{39}$. Infection with HIV-1 is not sufficient for obtaining malignant transformation. However, co-infection of HIV-1 and other viruses, e.g. KSHV, apparently play an important role in the initiation of neoplastic transformation ${ }^{34}$. 


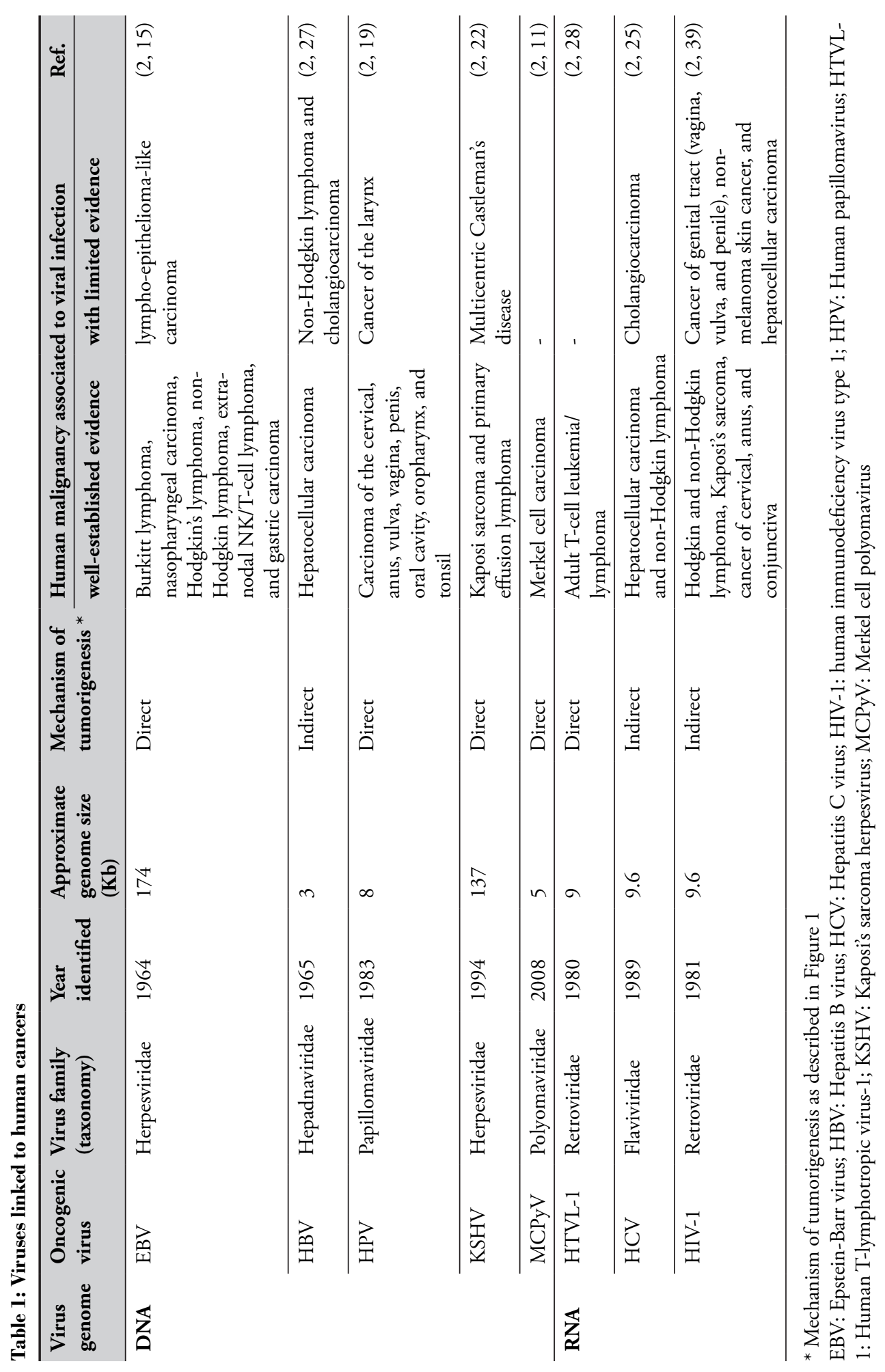




\section{Polyomaviruses}

In this thesis, the focus will be on polyomaviruses (PyVs), which are small, unenveloped, circular and double-strand DNA viruses with a size range between 4.5-5.4 kilobase (kb) that belong to the family of polyomaviridae, Figure $2^{40-42}$. The PyV family has been significantly expanded in the last decade and consists of more than 102 species capable of infecting mammals, birds, and fish as their host ${ }^{41,43,44}$. The murine polyomavirus $(\mathrm{MuPyV})$ was the firstly discovered polyomavirus in 1953, which proved to induce different types of tumors in animal models ${ }^{45}$, 46 . In 1953, Eddy et al. showed that injection of MPyV in mice, hamster, and rat newborns lead to different neoplasms ${ }^{47,48}$. In 1960, Sweet and Hilleman discovered the simian vacuolating virus 40 (SV40) in rhesus monkey kidney cells ${ }^{49}$. SV 40 was shown to be an oncogenic virus in animal models, but although SV40 DNA was found to be present in many different human tumors, it was neither considered a human polyomavirus nor as a human carcinogen ${ }^{8,50}$. Polyomaviruses have been shown to be strongly oncogenic in heterologous animal models, which point to the possibility that PyVs play a role in human diseases, although yet no decisive role has been proven ${ }^{45,51,52}$.

\section{Human Polyomaviruses}

JC polyomavirus (JCPyV) and BK polyomavirus (BKPyV) were the first human polyomaviruses isolated from patients in $1971{ }^{53,54}$. JCPyV was isolated from brain tissues and has been shown to cause progressive multifocal leukoencephalopathy (PML) in immunosuppressed patients ${ }^{54}$. BK was isolated from the urine of a renal allograft recipient and has been shown to cause hemorrhagic cystitis, also in immunosuppressed

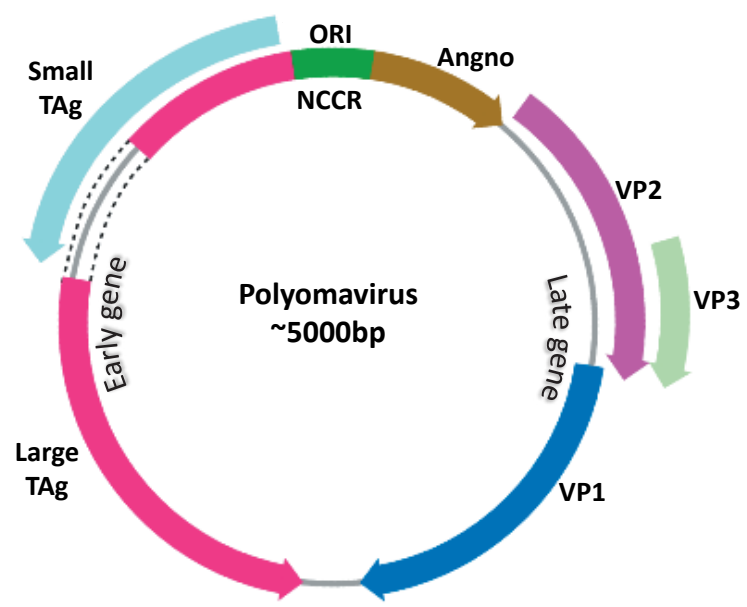

Figure 2: General features of polyomavirus. Circular double-strand DNA genome with non-enveloped capsid. The early gene contains small and large tumor antigens, while the late gene contains the three capsid structural proteins VP1, VP2, and VP3. (created in BioRender.com) 
patients ${ }^{53}$. JCPyV and $\mathrm{BKPyV}$ have been shown to induce cancer in rodent models and have the ability to target the tumor suppressor protein p 53 in a similar way as SV $40{ }^{51,52}$.

Since 2007, 13 new PyVs have been isolated from human specimens, and 10 of them were confirmed to be human polyomaviruses (HPyV's) Table 2. In 2007, two new members of the HPyVs family were identified, i.e. the Karolinska Institute polyomavirus (KIPyV) and the Washington University polyomavirus (WUPyV). Both KIPyV and WUPyV were isolated from nasopharyngeal aspirate (NPA) specimens of lower respiratory tract disease in children ${ }^{55,56}$. Yet, no human disease has been associated with one of the two viruses 55,56 .

In 2008, the fifth HPyV called Merkel cell polyomavirus (MCPyV) was discovered and linked to the pathogenesis of Merkel cell carcinoma (MCC) ${ }^{57}$. MCC is an extremely aggressive, relatively rare non-melanoma skin cancer of unknown cellular origin, affecting especially elderly and immunosuppressed patients ${ }^{11,58}$. MCPyV DNA was found to be present and clonally integrated into the tumor DNA in approximately $80 \%$ of MCCs. In addition, in MCC the large tumor antigen (LTAg) gene proved to carry point mutations introducing stop codons, leading to expression of a truncated helicase domain ${ }^{10,11}$. This renders a deficient virus replication and thus promotes the mutated $\mathrm{MCPyV}$ supertransforming properties. In particular, the binding of truncated LTAg to the retinoblastoma protein (pRB) maintains the replication capacity in MCPyV-positive cell lines Figure $3{ }^{42,59}$. In addition, MCPyV tumor antigen has been described to bind and inhibit the cell cycle regulator gene $\mathrm{p} 53$ gene to induce proliferation ${ }^{60}$.

Consequently, $\mathrm{MCPyV}$ has been categorized as "Group 2A carcinogen: probably carcinogenic to humans" carcinogen by the International Agency for Research on Cancer (IARC) in $2012^{8}$. Of interest, MCPyV-positive MCC seems to have a better response to treatment and a favorable survival rate compared to $\mathrm{MCPyV}$-negative MCC, which has been shown to be associated with UV damage and with a very high tumor mutational burden ${ }^{61-65}$. The finding of $\mathrm{MCPyV}$ in chronic lymphocytic leukemia (CLL) patient specimens, with and without a 246bp deletion in the helicase region of the LTAg, provided more evidence of the likely association of MCPyV with CLL ${ }^{66}$, as did the finding of $\mathrm{MCPyV}$ in neoplastic B cells e.g., in CLL cells. This opened the spectrum for further exploration of possibly MCPyV-associated human cancers ${ }^{66-68}$.

In 2010, three new HPyVs were discovered. Human polyomavirus 6 (HPyV6) and human polyomavirus 7 (HPyV7) were isolated simultaneously from healthy skin swabs using a modified rolling circle amplification (RCA) technique. The complete HPyV7 genome was found to share about $68 \%$ of HPyV6 nucleotides ${ }^{69}$. In addition, trichodysplasia spinulosa-associated polyomavirus (TSPyV) was isolated from plucked facial spines of a 


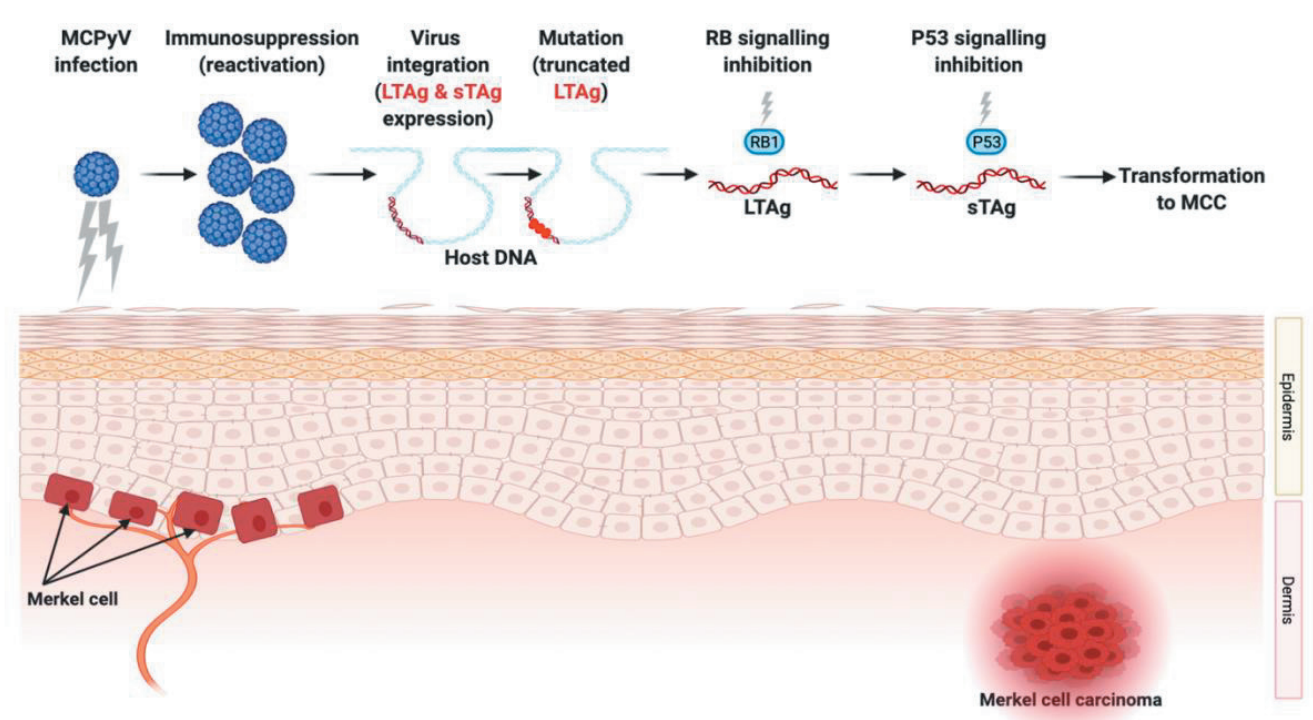

Figure 3: Schematic diagram showing MCPyV-induced Merkel cell carcinoma tumorigenesis. (created in BioRender.com)

heart transplant patient. TSPyV was found to be associated with a very rare skin condition called trichodysplasia spinulosa (TS) in an immunocompromised patient ${ }^{70}$. These newly discovered HPyVs raised the question about their potential role in the etiopathogenesis of other human diseases and cancers. Recently, literature reported the seroprevalence and prevalence of HPyV6 and 7 DNA in neoplastic diseases, such as melanoma, as well as in non-neoplastic diseases, such as chronic tonsillitis ${ }^{71-75}$.

In 2011, Human Polyomavirus 9 (HPyV9) was identified from different human specimens. Scuda et al. detected HPyV9 from a serum sample of a kidney transplant patient who was receiving an immunosuppressive treatment ${ }^{76}$. In addition, Sauvage et al. detected HPyV9 from a cutaneous swab obtained from an MCC patient ${ }^{77}$. However, several studies screened for HPyV9 in different tumor specimens, and no evidence was found for the presence of HPyV9 in diverse skin cancers including basal cell carcinoma, squamous cell carcinoma, keratoacanthoma, microcystic adnexal carcinoma, atypical fibroxanthoma, actinic keratosis, and mucosal melanoma ${ }^{78,79}$. Also, HPyV9 could not be detected in other neoplasms, such as CLL and glioblastoma ${ }^{80,81}$. Thus, no evidence of clinical relevance has been shown so far for the presence of HPyV9 in either serum or skin.

Later in 2012, both Malawi polyomavirus (MWPyV) and St Louis polyomavirus (STLPyV) were isolated from fecal samples of healthy children ${ }^{82-84}$. Furthermore, MWPyV (also known as HPyV10) was identified in the skin of a patient with warts, 
hypogammaglobulinemia, infections, and myelokathexis (WHIM) syndrome ${ }^{85}$. In addition, the prevalence of HPyV10 (MWPyV) was reported in diverse specimens other than stool ${ }^{86-88}$. Rockett et al. reported the presence of MWPyV in respiratory secretions of both symptomatic patients (no further details were reported) and healthy individuals ${ }^{86}$. In addition, MWPyV was detected in the tonsil and adenoid lymphoid tissues of immunocompetent children ${ }^{87,88}$. MWPyV tumor antigen (TAg) was found to bind with tumor suppressor proteins $\mathrm{p} 53$, pRB, and PP2A, however, the TAg was unable to promote cellular proliferation ${ }^{89}$. Likewise, STLPyV was isolated from stool specimens as well as other specimens including tonsillar tissues and the skin of condylomas patients ${ }^{88,90}$.

In 2013, human polyomavirus 12 (HPyV12) was initially isolated from liver specimens, then confirmed to be present in the colon and rectum specimens of patients with malignancy ${ }^{91}$. HPyV12 has no definitive association with a human disease yet. Indeed, a very similar sequence of HPyV12 was found to be commonly present in shrews ${ }^{92}$. It has been suggested that detecting HPyV12-positive human specimens was due to external artifact sources, such as laboratory contamination ${ }^{92-94}$. This is supported by the findings that $\mathrm{HPyV} 12$ was not detected in diverse specimens from different diseases ${ }^{93}$, 95-97. Therefore, the International Committee on Taxonomy of Viruses (ICTV) excluded HPyV12 from the list of HPyVs ${ }^{44}$.

In 2014, New Jersey polyomavirus (NJPyV) was isolated from vascular endothelial cells of a pancreatic transplant patient ${ }^{98}$. Indeed, this patient was reported to be sick two weeks after the exposure to the floodwaters of hurricane Sandy ${ }^{98}$. NJPyV tissue tropism and its role in human diseases are still not definitive. In addition, serological seroprevalence does not support that $\mathrm{NJPyV}$ is indeed infecting humans, as it is also not detected by metagenomic or serologic analyses in humans ${ }^{94}$. Of interest, NJPyV shows a closely related (70-80\%) sequence to a primate polyomavirus, i.e. the chimpanzee polyomavirus $(\mathrm{ChPyV})^{99}$.

In 2017, a new polyomavirus was identified in human skin swabs and named Lyon IARC PyV (LIPyV) ${ }^{100}$. Yet, similarly to HPyV12 and NJPyV, LIPyV has not been detected by metagenomic analyses in human specimens ${ }^{94}$. However, LIPyV was detected in cat faces and found in human specimens most likely due to contamination ${ }^{101}$. Recently, in 2019, Quebec polyomavirus (QPyV) was isolated from the stool of human patients by metagenomic analysis ${ }^{94}$. So far, only one study tested the presence of QPyV in urine, CSF, nasopharyngeal aspirates, and plasma specimens obtained from diverse nonneoplastic diseases, and revealed that QPyV-DNA is detected at a low frequency in the urine specimens of SLE patients, pregnant women, and multiple sclerosis patients ${ }^{102}$. However, no other data are available about QPyV and thus further confirmation of this virus in human tissues is required. 
Table 2 Polyomaviruses isolated from human and associated diseases

\begin{tabular}{|c|c|c|c|c|c|c|}
\hline$\#$ & $\begin{array}{l}\text { Polyomavirus } \\
\text { isolated from } \\
\text { human }\end{array}$ & $\begin{array}{l}\text { Genome } \\
\text { Size } \\
\text { (bp) }\end{array}$ & Isolated from & Associated disease & $\begin{array}{l}\text { Year of } \\
\text { discovery }\end{array}$ & Reference \\
\hline 1 & $\begin{array}{l}\text { BK polyomavirus } \\
(\mathrm{BKPyV})\end{array}$ & 5141 & Urine & $\begin{array}{l}\text { Nephropathy and } \\
\text { hemorrhagic cystitis }\end{array}$ & 1971 & (53) \\
\hline 2 & $\begin{array}{l}\text { JC polyomavirus } \\
(\mathrm{JCPyV})\end{array}$ & 5130 & $\begin{array}{l}\text { Brain tissue and } \\
\text { urine }\end{array}$ & $\begin{array}{l}\text { Progressive multifocal } \\
\text { leukoencephalopathy }\end{array}$ & 1971 & $(54)$ \\
\hline 3 & $\begin{array}{l}\text { Karolinska Institute } \\
\text { polyomavirus } \\
(\mathrm{KIPyV})\end{array}$ & 5040 & Respiratory tract & Not defined & 2007 & $(55)$ \\
\hline 4 & $\begin{array}{l}\text { Washington } \\
\text { University } \\
\text { polyomavirus } \\
\text { (WUPyV) }\end{array}$ & 5229 & Respiratory tract & Not defined & 2007 & (56) \\
\hline 5 & $\begin{array}{l}\text { Merkel cell } \\
\text { polyomavirus } \\
(\mathrm{MCPyV})\end{array}$ & 5387 & $\begin{array}{l}\text { Merkel cell } \\
\text { carcinoma }\end{array}$ & Merkel cell carcinoma & 2008 & (11) \\
\hline 6 & $\begin{array}{l}\text { Human } \\
\text { polyomavirus } 6 \\
\text { (HPyV6) }\end{array}$ & 4926 & Skin swabs & $\begin{array}{l}\text { Pruritic skin eruption in } \\
\text { immunocompromised }\end{array}$ & 2010 & (69) \\
\hline 7 & $\begin{array}{l}\text { Human } \\
\text { polyomavirus } 7 \\
(\mathrm{HPyV} 7)\end{array}$ & 4949 & Skin swabs & $\begin{array}{l}\text { Pruritic skin eruption in } \\
\text { immunocompromised }\end{array}$ & 2010 & (69) \\
\hline 8 & $\begin{array}{l}\text { Trichodysplasia } \\
\text { spinulosa } \\
\text { polyomavirus } \\
(\mathrm{TSPyV})\end{array}$ & 5232 & $\begin{array}{l}\text { Plucked facial } \\
\text { spines }\end{array}$ & $\begin{array}{l}\text { Trichodysplasia spinulosa } \\
\text { and pilomatrix dysplasia }\end{array}$ & 2010 & $(70)$ \\
\hline 9 & $\begin{array}{l}\text { Human } \\
\text { polyomavirus } 9 \\
(\mathrm{HPyV} 9)\end{array}$ & 5026 & $\begin{array}{l}\text { Skin, blood and } \\
\text { urine }\end{array}$ & Not defined & 2011 & $(76,77)$ \\
\hline 10 & $\begin{array}{l}\text { Malawi } \\
\text { polyomavirus } \\
(\mathrm{MWPyV}) / \mathrm{MX} \\
\text { polyomavirus } \\
(\mathrm{MXPyV}) / \\
\text { HPyV10 }\end{array}$ & 4939 & $\begin{array}{l}\text { Fecal samples and } \\
\text { wart }\end{array}$ & Not defined & 2012 & $(82,83,85)$ \\
\hline 11 & $\begin{array}{l}\text { St Louis } \\
\text { polyomavirus } \\
(\mathrm{STLPyV})\end{array}$ & 4776 & Fecal samples & Not defined & 2012 & (84) \\
\hline 12 & $\begin{array}{l}\text { Human } \\
\text { polyomavirus } 12 \\
(\mathrm{HPyV} 12)\end{array}$ & 5033 & $\begin{array}{l}\text { Liver and } \\
\text { gastrointestinal } \\
\text { tract }\end{array}$ & Not defined & 2013 & (91) \\
\hline 13 & $\begin{array}{l}\text { New Jersey } \\
\text { polyomavirus } \\
(\mathrm{NJPyV})\end{array}$ & 5108 & $\begin{array}{l}\text { Vascular muscle } \\
\text { endothelium }\end{array}$ & Not defined & 2014 & (98) \\
\hline 14 & $\begin{array}{l}\text { Lyon IARC PyV } \\
(\mathrm{LIPyV})\end{array}$ & 5269 & Skin swab & Not defined & 2017 & $(100)$ \\
\hline 15 & $\begin{array}{l}\text { Quebec } \\
\text { polyomavirus } \\
(\mathrm{QPyV})\end{array}$ & 4973 & Stool & Not defined & 2019 & (94) \\
\hline
\end{tabular}




\section{The aim and outline of this thesis:}

The etiopathogenesis and risk factors of many neoplasms are still unclear. In the last decade, the human polyomaviruses family has been expanded to $12 \mathrm{HPyVs}$ Figure 4. Yet, $\mathrm{MCPyV}$ is the only human tumor virus of the HPyVs family, and this raised significant attention to the potential role of other novel HPyVs in human tumorigenesis.

The aim of this thesis was to investigate and assess the potential contribution of known and novel HPyVs in a spectrum of human cancers. A broad spectrum of molecular techniques was used to test for the presence of these HPyVs. Chapter 2 reviews the current evidence to evaluate a possible role of the two closely related viruses HPyV6 and 7 in the etiopathogenesis of neoplastic human diseases. In chapter 3, the prevalence of $\mathrm{HPyV}$ in cholangiocarcinoma (CCA) tissues was investigated by screening for HPyV using diverse molecular techniques, including degenerate and virus-specific DNA PCR, FISH, RISH, and IHC. In chapter 4, the focus on the relation of BK virus-positive urinary cytology (presence of Decoy cells) to urothelial cell carcinoma (UCC) of the urinary bladder was assessed. In addition, the relation of positive $\mathrm{BKPyV}$ in the urine of UCC to a previously given intravesicular BCG or mitomycin immunotherapy treatment was evaluated. In chapter $\mathbf{5}$ it was investigated, if $\mathrm{MCPyV}$ plays a major role in the

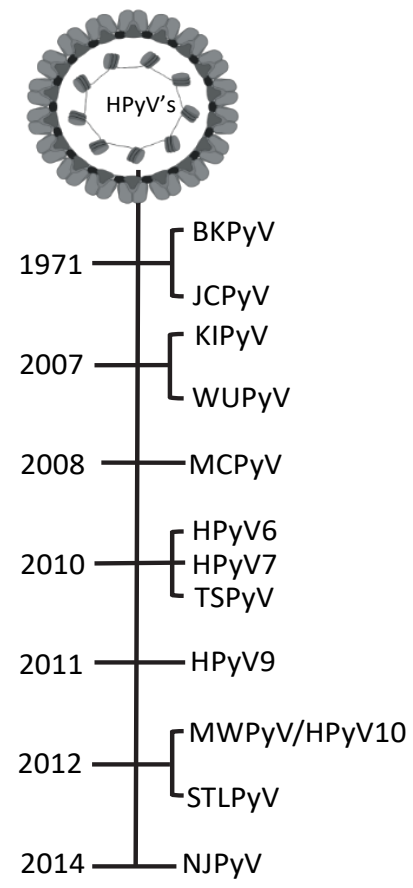

Figure 4: A Brief timeline showing the years of discovery of human polyomaviruses 
etiopathogenesis of human epithelial thymic tumors. For this purpose, diverse molecular techniques were applied to screen for $\mathrm{MCPyV}$ in a clinicopathologically well-characterized thymoma cohort, in which our research group has shown previously a high prevalence of HPyV7. In chapter 6, the presence of three different tumor viruses, i.e. MCPyV, human papillomavirus (HPV), and Epstein-Barr virus (EBV), were assessed in a unique large cohort of non-smokers and non-drinkers with head and neck squamous cell carcinoma (HNSCC) was performed. Chapter 7 discusses the findings and implications of this thesis and future perspectives about the role of HPyVs in human tumorigenesis. In addition, to address the overall strengths and limitations of methods and techniques of HPyVs screening. Finally, we provide a summary and the overall conclusions of our findings. 


\section{References}

1. Bray F, Ferlay J, Soerjomataram I, Siegel RL, Torre LA, Jemal A. Global cancer statistics 2018: GLOBOCAN estimates of incidence and mortality worldwide for 36 cancers in 185 countries. CA: a cancer journal for clinicians. 2018;68(6):394-424.

2. Wild CP WE, Stewart BW, editors. World Cancer Report: Cancer Research for Cancer Prevention. Lyon, France: International Agency for Research on Cancer Available from: http:// publicationsiarcfr/586 Licence: CC BY-NC-ND 30 IGO. 2020.

3. Lozano R, Naghavi M, Foreman K, Lim S, Shibuya K, Aboyans V, et al. Global and regional mortality from 235 causes of death for 20 age groups in 1990 and 2010: a systematic analysis for the Global Burden of Disease Study 2010. Lancet. 2012;380(9859):2095-128.

4. De Martel C, Georges D, Bray F, Ferlay J, Clifford GM. Global burden of cancer attributable to infections in 2018: a worldwide incidence analysis. The Lancet Global Health. 2020;8(2):e180-e90.

5. Bouvard V, Baan R, Straif K, Grosse Y, Secretan B, El Ghissassi F, et al. A review of human carcinogens_Part B: biological agents. The Lancet Oncology. 2009;10(4):321-2.

6. Plummer M, De Martel C, Vignat J, Ferlay J, Bray F, Franceschi S. Global burden of cancers attributable to infections in 2012: a synthetic analysis. The Lancet Global Health. 2016;4(9):e609-e16.

7. Biological agents. Volume 100 B. A review of human carcinogens. IARC Monogr Eval Carcinog Risks Hum. 2012;100(Pt B):1-441.

8. Bouvard V, Baan RA, Grosse Y, Lauby-Secretan B, El Ghissassi F, Benbrahim-Tallaa L, et al. Carcinogenicity of malaria and of some polyomaviruses. The Lancet Oncology. 2012;13(4):339-40.

9. Plummer M, Franceschi S, Vignat J, Forman D, De Martel C. Global burden of gastric cancer attributable toHelicobacterpylori. International journal of cancer. 2015;136(2):487-90.

10. Shuda M, Feng H, Kwun HJ, Rosen ST, Gjoerup O, Moore PS, et al. T antigen mutations are a human tumor-specific signature for Merkel cell polyomavirus. Proceedings of the National Academy of Sciences of the United States of America. 2008;105(42):16272-7.

11. Feng H, Shuda M, Chang Y, Moore PS. Clonal integration of a polyomavirus in human Merkel cell carcinoma. Science (New York, NY). 2008;319(5866):1096-100.

12. Zur Hausen H. Oncogenic DNA viruses. Oncogene. 2001;20(54):7820-3.

13. Moore PS, Chang Y. Why do viruses cause cancer? Highlights of the first century of human tumour virology. Nature reviews Cancer. 2010;10(12):878-89.

14. Kutok JL, Wang F. Spectrum of Epstein-Barr virus-associated diseases. Annu Rev Pathol. 2006;1:375404.

15. Epstein MA, Achong BG, Barr YM. VIRUS PARTICLES IN CULTURED LYMPHOBLASTS FROM BURKITT'S LYMPHOMA. Lancet. 1964;1(7335):702-3.

16. Bosch FX, Broker TR, Forman D, Moscicki AB, Gillison ML, Doorbar J, et al. Comprehensive control of human papillomavirus infections and related diseases. Vaccine. 2013;31 Suppl 7(Suppl 7):H1-31.

17. Duensing S, Lee LY, Duensing A, Basile J, Piboonniyom S, Gonzalez S, et al. The human papillomavirus type 16 E6 and E7 oncoproteins cooperate to induce mitotic defects and genomic instability by 
uncoupling centrosome duplication from the cell division cycle. Proceedings of the National Academy of Sciences of the United States of America. 2000;97(18):10002-7.

18. zur Hausen H. Viruses in human cancers. Science (New York, NY). 1991;254(5035):1167-73.

19. Dürst M, Gissmann L, Ikenberg H, zur Hausen H. A papillomavirus DNA from a cervical carcinoma and its prevalence in cancer biopsy samples from different geographic regions. Proceedings of the National Academy of Sciences of the United States of America. 1983;80(12):3812-5.

20. Boshart M, Gissmann L, Ikenberg H, Kleinheinz A, Scheurlen W, zur Hausen H. A new type of papillomavirus DNA, its presence in genital cancer biopsies and in cell lines derived from cervical cancer. Embo j. 1984;3(5):1151-7.

21. Cai Q, Verma SC, Lu J, Robertson ES. Molecular biology of Kaposi’s sarcoma-associated herpesvirus and related oncogenesis. Adv Virus Res. 2010;78:87-142.

22. Chang Y, Cesarman E, Pessin MS, Lee F, Culpepper J, Knowles DM, et al. Identification of herpesvirus-like DNA sequences in AIDS-associated Kaposi's sarcoma. Science (New York, NY). 1994;266(5192):1865-9.

23. Kassem A, Schopflin A, Diaz C, Weyers W, Stickeler E, Werner M, et al. Frequent detection of Merkel cell polyomavirus in human Merkel cell carcinomas and identification of a unique deletion in the VP1 gene. Cancer research. 2008;68(13):5009-13.

24. Beasley RP, Hwang LY, Lin CC, Chien CS. Hepatocellular carcinoma and hepatitis B virus. A prospective study of 22707 men in Taiwan. Lancet. 1981;2(8256):1129-33.

25. Choo QL, Kuo G, Weiner AJ, Overby LR, Bradley DW, Houghton M. Isolation of a cDNA clone derived from a blood-borne non-A, non-B viral hepatitis genome. Science (New York, NY). 1989;244(4902):359-62.

26. El-Serag HB, Mason AC. Risk factors for the rising rates of primary liver cancer in the United States. Arch Intern Med. 2000;160(21):3227-30.

27. Blumberg BS, Alter HJ, Visnich S. A "NEW" ANTIGEN IN LEUKEMIA SERA. Jama. 1965;191:541-6.

28. Poiesz BJ, Ruscetti FW, Gazdar AF, Bunn PA, Minna JD, Gallo RC. Detection and isolation of type C retrovirus particles from fresh and cultured lymphocytes of a patient with cutaneous T-cell lymphoma. Proceedings of the National Academy of Sciences of the United States of America. 1980;77(12):74159.

29. Matsuoka M, Jeang KT. Human T-cell leukemia virus type 1 (HTLV-1) and leukemic transformation: viral infectivity, Tax, HBZ and therapy. Oncogene. 2011;30(12):1379-89.

30. Balkwill FR, Mantovani A. Cancer-related inflammation: common themes and therapeutic opportunities. Semin Cancer Biol. 2012;22(1):33-40.

31. Coleman WB. Mechanisms of human hepatocarcinogenesis. Curr Mol Med. 2003;3(6):573-88.

32. Di Bisceglie AM. Hepatitis C and hepatocellular carcinoma. Hepatology. 1997;26(3 Suppl 1):34s-8s.

33. Brechot C, Kremsdorf D, Soussan P, Pineau P, Dejean A, Paterlini-Brechot P, et al. Hepatitis B virus (HBV)-related hepatocellular carcinoma (HCC): molecular mechanisms and novel paradigms. Pathologie-biologie. 2010;58(4):278-87. 
34. Engels EA. Non-AIDS-defining malignancies in HIV-infected persons: etiologic puzzles, epidemiologic perils, prevention opportunities. AIDS (London, England). 2009;23(8):875-85.

35. Chimal-Ramírez GK, Espinoza-Sánchez NA, Fuentes-Pananá EM. Protumor activities of the immune response: insights in the mechanisms of immunological shift, oncotraining, and oncopromotion. Journal of oncology. 2013;2013:835956.

36. Mantovani A, Allavena P, Sica A, Balkwill F. Cancer-related inflammation. Nature. 2008;454(7203):43644.

37. Elinav E, Nowarski R, Thaiss CA, Hu B, Jin C, Flavell RA. Inflammation-induced cancer: crosstalk between tumours, immune cells and microorganisms. Nature Reviews Cancer. 2013;13(11):759-71.

38. Tanaka Y, Hanada K, Mizokami M, Yeo AE, Shih JW, Gojobori T, et al. A comparison of the molecular clock of hepatitis $\mathrm{C}$ virus in the United States and Japan predicts that hepatocellular carcinoma incidence in the United States will increase over the next two decades. Proceedings of the National Academy of Sciences of the United States of America. 2002;99(24):15584-9.

39. Barré-Sinoussi F, Chermann JC, Rey F, Nugeyre MT, Chamaret S, Gruest J, et al. Isolation of a T-lymphotropic retrovirus from a patient at risk for acquired immune deficiency syndrome (AIDS). Science (New York, NY). 1983;220(4599):868-71.

40. Johne R, Buck CB, Allander T, Atwood WJ, Garcea RL, Imperiale MJ, et al. Taxonomical developments in the family Polyomaviridae. Archives of virology. 2011;156(9):1627-34.

41. Torres C. Evolution and molecular epidemiology of polyomaviruses. Infect Genet Evol. 2020;79:104150.

42. DeCaprio JA, Garcea RL. A cornucopia of human polyomaviruses. Nat Rev Microbiol. 2013;11(4):26476.

43. Moens U, Calvignac-Spencer S, Lauber C, Ramqvist T, Feltkamp MCW, Daugherty MD, et al. ICTV Virus Taxonomy Profile: Polyomaviridae. The Journal of general virology. 2017;98(6):1159-60.

44. ICTV-Report-pConsortium. Report on the taxonomy of the Polyomaviridae. Virus Taxonomy: 2018b Release. International Committee on Taxonomy of Viruses. 2019.

45. Ramqvist T, Dalianis T. Murine polyomavirus tumour specific transplantation antigens and viral persistence in relation to the immune response, and tumour development. Seminars in cancer biology. 2009;19(4):236-43.

46. Gross L. A filterable agent, recovered from Ak leukemic extracts, causing salivary gland carcinomas in C3H mice. Proc Soc Exp Biol Med. 1953;83(2):414-21.

47. Eddy BE, Stewart SE, Kirschstein RL, Young RD. Induction of subcutaneous nodules in rabbits with the SE polyoma virus. Nature. 1959;183(4663):766-7.

48. Eddy BE, Stewart SE. Characteristics of the SE polyoma virus. Am J Public Health Nations Health. 1959;49(11):1486-92.

49. Sweet BH, Hilleman MR. The vacuolating virus, S.V. 40. Proc Soc Exp Biol Med. 1960;105:420-7.

50. Butel JS, Lednicky JA. Cell and molecular biology of simian virus 40: implications for human infections and disease. Journal of the National Cancer Institute. 1999;91(2):119-34. 
51. Small JA, Khoury G, Jay G, Howley PM, Scangos GA. Early regions of JC virus and BK virus induce distinct and tissue-specific tumors in transgenic mice. Proceedings of the National Academy of Sciences of the United States of America. 1986;83(21):8288-92.

52. Dalrymple SA, Beemon KL. BK virus $\mathrm{T}$ antigens induce kidney carcinomas and thymoproliferative disorders in transgenic mice. Journal of virology. 1990;64(3):1182-91.

53. Gardner SD, Field AM, Coleman DV, Hulme B. New human papovavirus (B.K.) isolated from urine after renal transplantation. Lancet. 1971;1(7712):1253-7.

54. Padgett BL, Walker DL, ZuRhein GM, Eckroade RJ, Dessel BH. Cultivation of papova-like virus from human brain with progressive multifocal leucoencephalopathy. Lancet. 1971;1(7712):1257-60.

55. Allander T, Andreasson K, Gupta S, Bjerkner A, Bogdanovic G, Persson MA, et al. Identification of a third human polyomavirus. Journal of virology. 2007;81(8):4130-6.

56. Gaynor AM, Nissen MD, Whiley DM, Mackay IM, Lambert SB, Wu G, et al. Identification of a novel polyomavirus from patients with acute respiratory tract infections. PLoS pathogens. 2007;3(5):e64.

57. Wong SQ, Waldeck K, Vergara IA, Schroder J, Madore J, Wilmott JS, et al. UV-Associated Mutations Underlie the Etiology of MCV-Negative Merkel Cell Carcinomas. Cancer research. 2015;75(24):522834.

58. Toker C. Trabecular carcinoma of the skin. Archives of dermatology. 1972;105(1):107-10.

59. Houben R, Adam C, Baeurle A, Hesbacher S, Grimm J, Angermeyer S, et al. An intact retinoblastoma protein-binding site in Merkel cell polyomavirus large $\mathrm{T}$ antigen is required for promoting growth of Merkel cell carcinoma cells. International journal of cancer. 2012;130(4):847-56.

60. Cheng J, Rozenblatt-Rosen O, Paulson KG, Nghiem P, DeCaprio JA. Merkel cell polyomavirus large $\mathrm{T}$ antigen has growth-promoting and inhibitory activities. Journal of virology. 2013;87(11):6118-26.

61. Eid M, Nguyen J, Brownell I. Seeking Standards for the Detection of Merkel Cell Polyomavirus and its Clinical Significance. The Journal of investigative dermatology. 2017;137(4):797-9.

62. Moshiri AS, Doumani R, Yelistratova L, Blom A, Lachance K, Shinohara MM, et al. PolyomavirusNegative Merkel Cell Carcinoma: A More Aggressive Subtype Based on Analysis of 282 Cases Using Multimodal Tumor Virus Detection. The Journal of investigative dermatology. 2017;137(4):819-27.

63. Schrama D, Peitsch WK, Zapatka M, Kneitz H, Houben R, Eib S, et al. Merkel cell polyomavirus status is not associated with clinical course of Merkel cell carcinoma. The Journal of investigative dermatology. 2011;131(8):1631-8.

64. Harms KL, Zhao L, Johnson B, Wang X, Carskadon S, Palanisamy N, et al. Virus-positive Merkel Cell Carcinoma Is an Independent Prognostic Group with Distinct Predictive Biomarkers. Clinical cancer research : an official journal of the American Association for Cancer Research. 2021;27(9):2494-504.

65. Björn Andtback H, Björnhagen-Säfwenberg V, Shi H, Lui WO, Masucci GV, Villabona L. Sex Differences in Overall Survival and the Effect of Radiotherapy in Merkel Cell Carcinoma-A Retrospective Analysis of a Swedish Cohort. Cancers. 2021;13(2).

66. Pantulu ND, Pallasch CP, Kurz AK, Kassem A, Frenzel L, Sodenkamp S, et al. Detection of a novel truncating Merkel cell polyomavirus large $\mathrm{T}$ antigen deletion in chronic lymphocytic leukemia cells. Blood. 2010;116(24):5280-4. 
67. Toracchio S, Foyle A, Sroller V, Reed JA, Wu J, Kozinetz CA, et al. Lymphotropism of Merkel cell polyomavirus infection, Nova Scotia, Canada. Emerging infectious diseases. 2010;16(11):1702-9.

68. Teman CJ, Tripp SR, Perkins SL, Duncavage EJ. Merkel cell polyomavirus (MCPyV) in chronic lymphocytic leukemia/small lymphocytic lymphoma. Leuk Res. 2011;35(5):689-92.

69. Schowalter RM, Pastrana DV, Pumphrey KA, Moyer AL, Buck CB. Merkel cell polyomavirus and two previously unknown polyomaviruses are chronically shed from human skin. Cell host \& microbe. 2010;7(6):509-15.

70. van der Meijden E, Janssens RW, Lauber C, Bouwes Bavinck JN, Gorbalenya AE, Feltkamp MC. Discovery of a new human polyomavirus associated with trichodysplasia spinulosa in an immunocompromized patient. PLoS pathogens. 2010;6(7):e1001024.

71. Schrama D, Groesser L, Ugurel S, Hafner C, Pastrana DV, Buck CB, et al. Presence of human polyomavirus 6 in mutation-specific BRAF inhibitor-induced epithelial proliferations. JAMA dermatology. 2014;150(11):1180-6.

72. Imajoh M, Hashida Y, Nakajima H, Sano S, Daibata M. Prevalence and viral DNA loads of three novel human polyomaviruses in skin cancers from Japanese patients. The Journal of dermatology. 2013;40(8):657-60.

73. Sadeghi M, Wang Y, Ramqvist T, Aaltonen LM, Pyoria L, Toppinen M, et al. Multiplex detection in tonsillar tissue of all known human polyomaviruses. BMC infectious diseases. 2017;17(1):409.

74. Herberhold S, Hellmich M, Panning M, Bartok E, Silling S, Akgul B, et al. Human polyomavirus and human papillomavirus prevalence and viral load in non-malignant tonsillar tissue and tonsillar carcinoma. Medical microbiology and immunology. 2017;206(2):93-103.

75. Salakova M, Koslabova E, Vojtechova Z, Tachezy R, Sroller V. Detection of human polyomaviruses $\mathrm{MCPyV}, \mathrm{HPyV} 6$, and HPyV7 in malignant and non-malignant tonsillar tissues. Journal of medical virology. 2016;88(4):695-702.

76. Scuda N, Hofmann J, Calvignac-Spencer S, Ruprecht K, Liman P, Kuhn J, et al. A novel human polyomavirus closely related to the african green monkey-derived lymphotropic polyomavirus. Journal of virology. 2011;85(9):4586-90.

77. Sauvage V, Foulongne V, Cheval J, Ar Gouilh M, Pariente K, Dereure O, et al. Human polyomavirus related to African green monkey lymphotropic polyomavirus. Emerging infectious diseases. 2011;17(8):1364-70.

78. Scola N, Wieland U, Silling S, Altmeyer P, Stucker M, Kreuter A. Prevalence of human polyomaviruses in common and rare types of non-Merkel cell carcinoma skin cancer. The British journal of dermatology. 2012;167(6):1315-20.

79. Ramqvist T, Nordfors C, Dalianis T, Ragnarsson-Olding B. DNA from human polyomaviruses, TSPyV, MWPyV, HPyV6, 7 and 9 was not detected in primary mucosal melanomas. Anticancer research. 2014;34(2):639-43.

80. Imajoh M, Hashida Y, Taniguchi A, Kamioka M, Daibata M. Novel human polyomaviruses, Merkel cell polyomavirus and human polyomavirus 9, in Japanese chronic lymphocytic leukemia cases. Journal of hematology \& oncology. 2012;5:25. 
81. Hashida Y, Taniguchi A, Yawata T, Hosokawa S, Murakami M, Hiroi M, et al. Prevalence of human cytomegalovirus, polyomaviruses, and oncogenic viruses in glioblastoma among Japanese subjects. Infectious agents and cancer. 2015;10:3.

82. Yu G, Greninger AL, Isa P, Phan TG, Martinez MA, de la Luz Sanchez M, et al. Discovery of a novel polyomavirus in acute diarrheal samples from children. PloS one. 2012;7(11):e49449.

83. Siebrasse EA, Reyes A, Lim ES, Zhao G, Mkakosya RS, Manary MJ, et al. Identification of MW polyomavirus, a novel polyomavirus in human stool. Journal of virology. 2012;86(19):10321-6.

84. Lim ES, Reyes A, Antonio M, Saha D, Ikumapayi UN, Adeyemi M, et al. Discovery of STL polyomavirus, a polyomavirus of ancestral recombinant origin that encodes a unique $\mathrm{T}$ antigen by alternative splicing. Virology. 2013;436(2):295-303.

85. Buck CB, Phan GQ, Raiji MT, Murphy PM, McDermott DH, McBride AA. Complete genome sequence of a tenth human polyomavirus. Journal of virology. 2012;86(19):10887.

86. Rockett RJ, Sloots TP, Bowes S, O'Neill N, Ye S, Robson J, et al. Detection of novel polyomaviruses, TSPyV, HPyV6, HPyV7, HPyV9 and MWPyV in feces, urine, blood, respiratory swabs and cerebrospinal fluid. PloS one. 2013;8(5):e62764.

87. Papa N, Zanotta N, Knowles A, Orzan E, Comar M. Detection of Malawi polyomavirus sequences in secondary lymphoid tissues from Italian healthy children: a transient site of infection. Virology journal. 2016;13:97.

88. Peng J, Li K, Zhang C, Jin Q. MW polyomavirus and STL polyomavirus present in tonsillar tissues from children with chronic tonsillar disease. 2016;22(1):97.e1-.e3.

89. Berrios C, Jung J, Primi B, Wang M, Pedamallu C, Duke F, et al. Malawi polyomavirus is a prevalent human virus that interacts with known tumor suppressors. Journal of virology. 2015;89(1):857-62.

90. Pastrana DV, Fitzgerald PC, Phan GQ, Raiji MT, Murphy PM, McDermott DH, et al. A divergent variant of the eleventh human polyomavirus species, saint louis polyomavirus. Genome announcements. 2013;1(5).

91. Korup S, Rietscher J, Calvignac-Spencer S, Trusch F, Hofmann J, Moens U, et al. Identification of a novel human polyomavirus in organs of the gastrointestinal tract. PloS one. 2013;8(3):e58021.

92. Gedvilaite A, Tryland M, Ulrich RG, Schneider J, Kurmauskaite V, Moens U, et al. Novel polyomaviruses in shrews (Soricidae) with close similarity to human polyomavirus 12 . The Journal of general virology. 2017;98(12):3060-7.

93. Moens U, Prezioso C, Pietropaolo V. Genetic Diversity of the Noncoding Control Region of the Novel Human Polyomaviruses. Viruses. 2020;12(12).

94. Ondov BD, Starrett GJ, Sappington A, Kostic A, Koren S, Buck CB, et al. Mash Screen: highthroughput sequence containment estimation for genome discovery. Genome biology. 2019;20(1):232.

95. Kourieh A, Combes JD, Tommasino M, Dalstein V, Clifford GM, Lacau St Guily J, et al. Prevalence and risk factors of human polyomavirus infections in non-malignant tonsils and gargles: the SPLIT study. The Journal of general virology. 2018;99(12):1686-98.

96. Daprà V, Galliano I, Rassu M, Calvi C, Montanari P, Merlino C, et al. Lack of detection of HPyV12 DNA using real-time PCR in Italian infants with diarrhea. Minerva Pediatr. 2020. 
97. Bergallo M, Daprà V, Fava P, Ponti R, Calvi C, Montanari P, et al. DNA from Human Polyomaviruses, MWPyV, HPyV6, HPyV7, HPyV9 and HPyV12 in Cutaneous T-cell Lymphomas. Anticancer research. 2018;38(7):4111-4.

98. Mishra N, Pereira M, Rhodes RH, An P, Pipas JM, Jain K, et al. Identification of a Novel Polyomavirus in a Pancreatic Transplant Recipient With Retinal Blindness and Vasculitic Myopathy. The Journal of infectious diseases. 2014.

99. Scuda N, Madinda NF, Akoua-Koffi C, Adjogoua EV, Wevers D, Hofmann J, et al. Novel polyomaviruses of nonhuman primates: genetic and serological predictors for the existence of multiple unknown polyomaviruses within the human population. PLoS pathogens. 2013;9(6):e1003429.

100. Gheit T, Dutta S, Oliver J, Robitaille A, Hampras S, Combes JD, et al. Isolation and characterization of a novel putative human polyomavirus. Virology. 2017;506:45-54.

101. Fahsbender E, Altan E, Estrada M, Seguin MA, Young P, Leutenegger CM, et al. Lyon-IARC Polyomavirus DNA in Feces of Diarrheic Cats. Microbiol Resour Announc. 2019;8(29).

102. Prezioso C, Van Ghelue M, Pietropaolo V, Moens U. Detection of Quebec Polyomavirus DNA in Samples from Different Patient Groups. Microorganisms. 2021;9(5):1082. 
CHAPTER 2

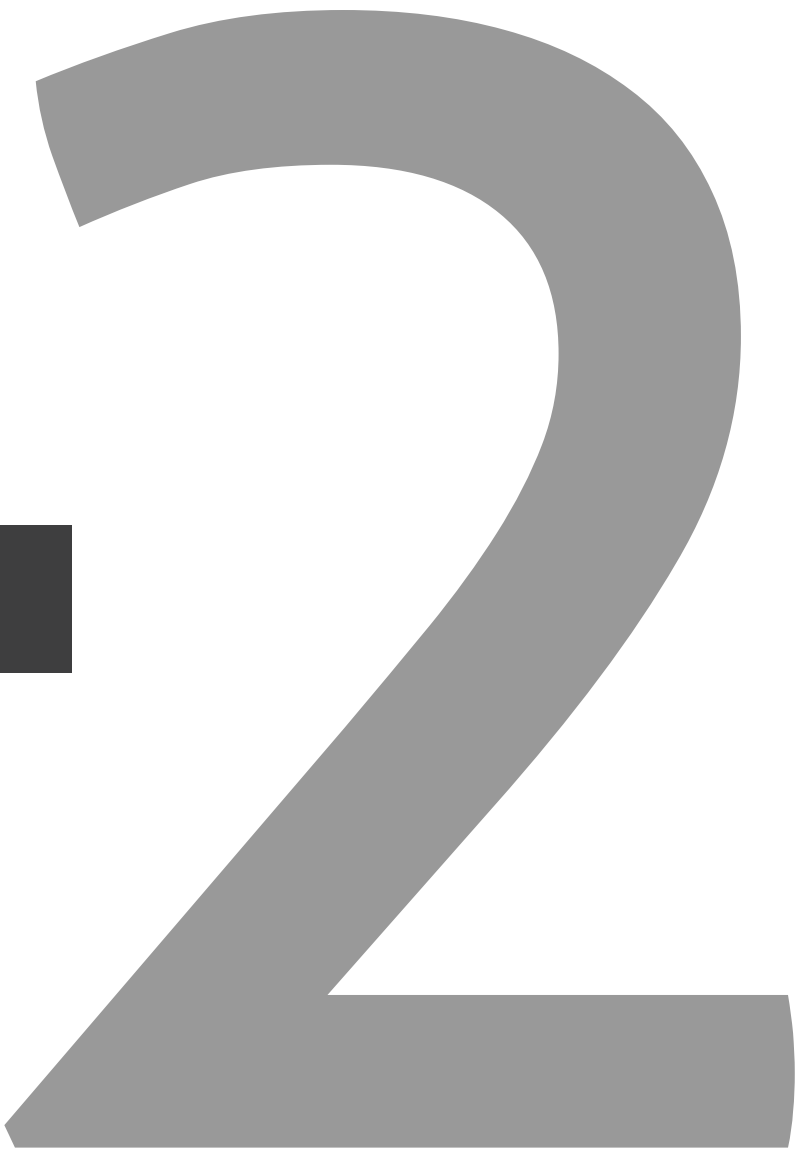




\section{Emerging Role of Human Polyomaviruses 6 and 7 in Human Cancers}

Faisal Klufah, Ghalib Mobaraki, Dan Liu, Raed A. Alharbi,

Anna Kordelia Kurz, Ernst Jan M. Speel, Véronique Winnepenninckx, and Axel zur Hausen 


\section{ABSTRACT}

\section{Background}

Currently 12 human polyomaviruses (HPyVs) have been identified, 6 of which have been associated with human diseases, including cancer. The discovery of the Merkel cell polyomavirus and its role in the etiopathogenesis in the majority of Merkel cell carcinomas has drawn significant attention, also to other novel HPyVs. In 2010, HPyV6 and HPyV7 were identified in healthy skin swabs. Ever since it has been speculated that they might contribute to the etiopathogenesis of skin and non-cutaneous human cancers.

\section{Main body}

Here we comprehensively reviewed and summarized the current evidence potentially indicating an involvement of HPyV6 and HPyV7 in the etiopathogenesis of neoplastic human diseases. The seroprevalence of both HPyV6 and 7 is high in a normal population and increases with age. In skin cancer tissues, HPyV6- DNA was far more often prevalent than HPyV7 in contrast to cancers of other anatomic sites, in which HPyV7 DNA was more frequently detected.

\section{Conclusion}

It is remarkable to find that the detection rate of HPyV6-DNA in tissues of skin malignancies is higher than HPyV7-DNA and may indicate a role of HPyV6 in the etiopathogenesis of the respected skin cancers. However, the sheer presence of viral DNA is not enough to prove a role in the etiopathogenesis of these cancers.

\section{KEYWORDS}

HPyV6, HPyV7, Merkel cell polyomavirus, oncogenic viruses, cutaneous tumors, viral persistence, tumor virus. 


\section{Background}

Polyomaviruses (PyV's) comprise a family of non-enveloped viruses containing a small genome of approximately $5.0 \mathrm{kbp}$ in size of circular double-stranded DNA and are capable of infecting mammals and birds ${ }^{1,2,3}$. PyV's are strongly oncogenic in heterologous animal models, suggesting that next to human also non-human polyomaviruses could possibly play a role in human cancers ${ }^{4,5,6,7}$. To date, fifteen novel PyV's have been isolated from different human specimens ${ }^{8}$. In 2019, the International Committee on Taxonomy of Viruses (ICTV) reported only twelve human polyomaviruses (HPyVs) after having HPyV12 excluded, which was shown to be infecting shrews ${ }^{9,}{ }^{10}$. In addition, the Lyon IARC polyomavirus which initially was isolated from human skin swab in 2017 is also found in cats feces ${ }^{11}$. Recently in 2019, Ondov et al. isolated the Quebec PyV from a stool sample of one patient through the MinHash algorithm ${ }^{12}$. Yet it still has not been listed as a human polyomavirus by the ICTV ${ }^{9}$. Six of the identified HPyVs have been associated with human diseases, including cancer ${ }^{3,8,13,14}$.

In 2008, Merkel cell polyomavirus (MCPyV) has been linked to the etiopathogenesis of the majority (i.e. $80 \%$ ) of the highly aggressive neuroendocrine Merkel cell carcinoma (MCC) ${ }^{15,16,17}$. Already in $2012 \mathrm{MCPyV}$ has been categorized as a group $2 \mathrm{~A}$ carcinogen by the International Agency for Research on Cancer (IARC) ${ }^{18}$. MCPyV has also been detected in non-neoplastic B cells (e.g. reactive hyperplasia and normal lymph node) and neoplastic B cells (e.g. chronic lymphocytic leukemia cells,) suggesting a role for $\mathrm{MCPyV}$ in B-cell neoplasias $15,19,20,21,22$.

In 2010, HPyV6 and HPyV7 were identified from healthy skin swabs using the rolling circle amplification technique ${ }^{23}$. The HPyV7 genome shares approximately $68 \%$ sequence identity with $\mathrm{HPyV}^{23}$. As these novel HPyVs were discovered in the skin, their presence in the skin and non-cutaneous human cancers, similar to MCPyV has been studied intensively $24,25,26,27,28,29,30,31$. Additionally, HPyV6 and 7 proteins have been suggested to play a key role in the binding and deactivation of $\mathrm{p} 53^{32}$. The oncogenic role of HPyVs in general has been reviewed extensively $3,7,13,33,34,35,36$. By reviewing sero-epidemiological and tumor-pathological evidence, we here focus on the possible oncogenic role of HPyV6 and 7 in human cancers, and in particular their direct or indirect contribution to tumorigenesis.

\section{Seroprevalence of HPyV6 and HPyV7 in healthy individuals}

Several studies have assessed HPyV6 and HPyV7 serum antibody levels across a broad range of age groups of various populations $23,37,38,39,40,41$. In 2010, 95 sera from blood donors were tested for the seroprevalence of $\mathrm{HPyVs}$ and revealed higher seropositivity for HPyV6 (69\%) than HPyV7 (35\%) using direct enzyme-linked immunosorbent assays 
(ELISA) directed against virus-like particles (VLP) based on viral protein 1 (VP1) capsid ${ }^{23}$. Likewise, by using ELISA against VP1-VLP, another study estimated the seroprevalence of age groups ranging from 20 years and older and concluded that the prevalence of HPyV6 increased with age and was higher than that of HPyV7 (80-92\% vs 60-80\%, respectively) ${ }^{37}$. The HPyVs seroprevalence of 1050 Dutch blood donors aged from 18 to 69 was determined and showed that the prevalence of both viruses increased with age, with 84 and $72 \%$ seropositivity for HPyV6 and HPyV7 VP1 antibodies, respectively ${ }^{38}$. Nicol et al. assessed the seroprevalence of both viruses in 828 subjects across different age groups starting from age one using VLP-based direct ELISA. Their study found that HPyV6 (76\%) was more prevalent than HPyV7 (53\%) across all age groups ${ }^{39}$. Noteworthy, by performing Luminex xMAP technology to detect VP1antibodies for both viruses were detected in the early infancy group up to an age of 6 months with high frequency ( $80.1 \%$ for HPyV6 and $58.1 \%$ for HPyV7, respectively). On the other hand, a decline in the prevalence of both viruses was found for children age 6 months to 14 years old group ${ }^{40}$.

Altogether, both HPyV6 and 7 were found to be ubiquitous and to infect all age groups, and HPyV6 seroprevalence is slightly higher than HPyV7. Seroprevalence significantly increases with age, and a substantial proportion of individuals 50 years of age and older has tested positive for HPyV6 and HPyV7. Approximately 52-93\% of humans are seropositive for $\mathrm{HPyV6}$, whereas 33-84\% are seropositive for $\mathrm{HPyV7}$ according to the seroprevalence studies Table 1 23, 37,38,39,40,42, 43. Summarizing the overall age-specific seropositivity of HPyV6 and HPyV7 using ELISA or Luminex platform-based method was shown in (Fig. 1).

\section{Age-specific seroprevalence}

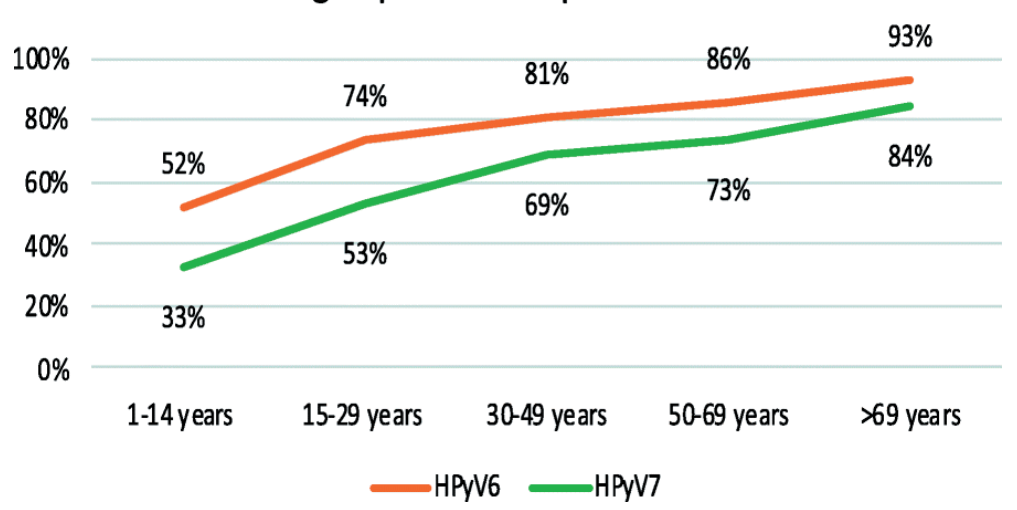

Fig. 1 Summarizing age-specific seroprevalence overall positivity of HPyV6 and HPyV7 using ELISA or Luminex platform-based methods to analyze antibodies against virus-like particles and/or glutathione-Stransferase consisting $\mathrm{HPyV} 6$ and 7 viral protein 
Table 1 Seroprevalence of HPyV6 and HPyV7 antibodies in serum using ELISA or Luminex platform

\begin{tabular}{|c|c|c|c|c|c|c|c|c|}
\hline \multirow{2}{*}{$\begin{array}{l}\text { Source of the } \\
\text { specimen }\end{array}$} & \multirow[t]{2}{*}{ Age (y) } & \multirow{2}{*}{$\begin{array}{l}\text { Samples } \\
\text { n }\end{array}$} & \multicolumn{2}{|c|}{ HPyV6 Positive n (\%) } & \multicolumn{2}{|c|}{ HPyV7 Positive n (\%) } & \multirow[t]{2}{*}{ Assay } & \multirow[t]{2}{*}{ Reference } \\
\hline & & & VLP & GST & VLP & GST & & \\
\hline \multirow{11}{*}{$\begin{array}{l}18-65(y) \text { Blood } \\
\text { donors. } \\
<18 \text { or }>65(y) \\
\text { From discarded } \\
\text { lab samples }\end{array}$} & $1-4$ & 48 & $18(37.5)$ & & $5(10.4)$ & & \multirow[t]{11}{*}{ ELISA } & \multirow[t]{11}{*}[39]{} \\
\hline & $5-9$ & 69 & $40(57.9)$ & & $23(33.3)$ & & & \\
\hline & $10-14$ & 92 & $65(70.6)$ & & $41(44.6)$ & & & \\
\hline & $15-19$ & 89 & $55(61.8)$ & & $32(36)$ & & & \\
\hline & $20-29$ & 49 & $43(87.8)$ & & $22(44.9)$ & & & \\
\hline & $30-39$ & 73 & $49(67.1)$ & & $31(42.5)$ & & & \\
\hline & $40-49$ & 105 & $89(84.8)$ & & $62(59.1)$ & & & \\
\hline & $50-59$ & 95 & $78(82.1)$ & & $64(67.4)$ & & & \\
\hline & $60-69$ & 100 & $88(88)$ & & $68(68)$ & & & \\
\hline & $70-79$ & 52 & $46(88.5)$ & & $42(80.8)$ & & & \\
\hline & $\geq 80$ & 56 & $55(98.2)$ & & $48(85.7)$ & & & \\
\hline \multirow[t]{5}{*}{ Blood donors } & $20-29$ & 96 & $82(85.4)$ & $77(80.2)$ & $58(60.4)$ & $68(70.8)$ & \multirow[t]{5}{*}{ ELISA } & \multirow[t]{5}{*}[37]{} \\
\hline & $30-39$ & 129 & $114(88.4)$ & $111(86)$ & $92(71.3)$ & $106(82.2)$ & & \\
\hline & $40-49$ & 52 & $48(92.3)$ & $48(92.3)$ & $32(61.5)$ & $40(76.9)$ & & \\
\hline & $50-59$ & 24 & $22(91.7)$ & $21(87.5)$ & $16(66.7)$ & $19(79.2)$ & & \\
\hline & $>59$ & 5 & $4(80)$ & $4(80)$ & $3(60)$ & $4(80)$ & & \\
\hline \multirow{14}{*}{$\begin{array}{l}\text { Serum obtained } \\
\text { for routine lab } \\
\text { tests }\end{array}$} & $0-0.5$ & 31 & & $25(80.6)$ & & $18(58.1)$ & \multirow{14}{*}{$\begin{array}{l}\text { Luminex } \\
\text { platform }\end{array}$} & \multirow[t]{14}{*}[40]{} \\
\hline & $0.6-1.9$ & 63 & & $14(22.2)$ & & $12(19)$ & & \\
\hline & $2-3$ & 62 & & $21(33.9)$ & & $12(19.4)$ & & \\
\hline & $4-5$ & 58 & & $26(44.8)$ & & $19(32.8)$ & & \\
\hline & $6-7$ & 58 & & $29(50)$ & & $21(36.2)$ & & \\
\hline & $8-9$ & 70 & & $44(62.9)$ & & $27(38.6)$ & & \\
\hline & $10-14$ & 55 & & $31(56.4)$ & & $21(38.2)$ & & \\
\hline & $15-19$ & 59 & & $38(64.4)$ & & $25(42.4)$ & & \\
\hline & $20-29$ & 59 & & $39(66.1)$ & & $30(50.8)$ & & \\
\hline & $30-39$ & 64 & & $41(64.1)$ & & $35(54.7)$ & & \\
\hline & $40-49$ & 54 & & $34(63)$ & & $33(61.1)$ & & \\
\hline & $50-59$ & 58 & & $46(79.3)$ & & $36(62.1)$ & & \\
\hline & $60-69$ & 54 & & $50(92.6)$ & & $45(83.3)$ & & \\
\hline & $>70$ & 54 & & $50(92.6)$ & & $46(85.2)$ & & \\
\hline \multirow[t]{5}{*}{ Blood donors } & $18-29$ & 208 & & $156(75.7)$ & & $117(56.8)$ & \multirow{5}{*}{$\begin{array}{l}\text { Luminex } \\
\text { platform }\end{array}$} & \multirow[t]{5}{*}[38]{} \\
\hline & $30-39$ & 209 & & $175(84.5)$ & & $154(74.4)$ & & \\
\hline & $40-49$ & 208 & & $173(83.6)$ & & $155(74.9)$ & & \\
\hline & $50-59$ & 215 & & $184(85.6)$ & & $154(71.6)$ & & \\
\hline & $60-69$ & 210 & & $187(89.5)$ & & $169(80.9)$ & & \\
\hline \multicolumn{2}{|l|}{ Blood donors } & 95 & $66(69)$ & & $33(35)$ & & & {$[23]$} \\
\hline $\begin{array}{l}\text { SCCs } \\
\text { transplanted cases }\end{array}$ & & 110 & & $65(59)$ & & $57(51.8)$ & \multirow{2}{*}{$\begin{array}{l}\text { ELISA } \\
\text { and } \\
\text { Luminex } \\
\text { platform }\end{array}$} & \multirow[t]{2}{*}[42]{} \\
\hline $\begin{array}{l}\text { Non-SCCs } \\
\text { transplanted cases }\end{array}$ & & 207 & & $137(66.2)$ & & $85(41)$ & & \\
\hline \multicolumn{2}{|l|}{ Lung cancer } & 511 & $393(76.9)$ & & $\begin{array}{l}333 \\
(65.2)\end{array}$ & & \multirow[t]{2}{*}{$\begin{array}{l}\text { Luminex } \\
\text { platform }\end{array}$} & {$[43]$} \\
\hline Controls & & 508 & $400(78.7)$ & & $\begin{array}{l}334 \\
(65.8)\end{array}$ & & & \\
\hline
\end{tabular}

ELISA enzyme-linked immunosorbent assays, GST glutathione-S transferase, SCC squamous cell carcinoma, $V L P$ virus-like particles, $(y)$ years old 
In general, HPyV6 and 7 infections seem to be clinically rather asymptomatic, irrespective of the age at which the infection occurs. Increasing seroprevalence of these viruses is clearly age-related and reaches its peak in individuals beyond 40 years of age, possibly indicating a relationship of $\mathrm{HPyV} 6$ and 7 infections with immunosenescence. Regarding the methodology, the previous studies reported the seroprevalence of HPyV6 and HPyV7 in the sera obtained from blood donors or routine lab tests in various studies. Most of the studies used ELISA or Luminex platform antibody-binding assay methods to detect the antigen containing the structural viral capsid protein of the targeted viruses fused with VLP or glutathione S-transferase (GST) proteins Table 1. Moreover, one study assessed the agreement and sensitivity of both VLP and GST-based ELISA methods in detecting seropositivity ${ }^{37}$. The results showed only minor discrepancies in the seroprevalence comparing these two detection methods ${ }^{37}$. Kamminga et al. reported high seropositivity of HPyV6 and 7 by using a Luminex xMAP assay directed against the VP1 capsid of HPyVs, similar to other ELISA-based studies ${ }^{37,39,40,41}$.

\section{Seroprevalence of HPyV6 and HPyV7 in cancer patients}

Only a very limited number of studies have reported seroprevalence data of HPyV6 and 7 in cancer patients. Madeleine et al. recently reported that the seroprevalence of HPyV6 in immunosuppressed skin cancer patients $(59.1 \%)$ was lower compared to healthy controls $(66.2 \%)$, whereas the seroprevalence of $\mathrm{HPyV} 7$ in the same patient cohort was slightly elevated $(52 \%)$ compared to the control group (41\%) ${ }^{42}$. It is important to note that the ELISA GST detection method used by Madeleine et al. yielded a much lower seroprevalence compared to other studies using the same technique. Similarly, serum from non-smoking-related lung cancer patients showed positivity for HPyV6 (76.9\%) and HPyV7 (65.2\%), which was comparable to the seroprevalence of these viruses in normal population age groups older than 50 years ${ }^{43}$. HPyV6 and 7 seropositivity data and results, including methodology, are summarized in Table 1.

In the past, studies on the seroprevalence of other human tumor viruses, e.g. Epstein-Barr virus or human papillomaviruses (HPV), have helped to indirectly indicate whether or not these viruses are involved in the etiopathogenesis of human cancers ${ }^{44,45,46,47,48}$. Moreover, the infection by small DNA tumor viruses at an early age, such as high-risk HPV, has been identified as a potential cause of human cancer decades after infection ${ }^{47,48,49}$. Also, HPyV6 and 7 antibodies are already detectable in the sera of healthy individuals at an early age Table 1 indicating that a substantial number of individuals - if not the majority-acquire both viruses already during their early lifetime, which might be a prerequisite for the onset of a possibly associated disease or cancer later in life. 


\section{Prevalence of HPyV6 and HPyV7 DNA in non-neoplastic diseases}

A limited number of studies have screened for the prevalence of HPyV6- and HPyV7DNA in non-neoplastic diseases, with very low prevalence across a variety of specimens Table 2. Both HPyVs were reported in Kimura's disease-a chronic inflammatory disorder associated with lymphadenopathy-with $4 / 5$ and $1 / 5$ samples found to be positive for HPyV6- and HPyV7-DNA, respectively ${ }^{50}$. Only HPyV6-DNA was detected in lichen simplex chronicus (1/13 samples), Kikuchi disease, i.e. histiocytic necrotizing lymphadenitis (3/8 samples), dermatopathic lymph-adenitis (1/28 samples), and angiolymphoid hyperplasia with eosinophilia (ALHE) (4/5 samples) ${ }^{24,54,55}$. In addition, only one study showed the presence of HPyV6-DNA in lymphocytes of lymph nodes tissue from ALHE case by fluorescence in situ hybridization (FISH) ${ }^{54}$.

Studies conducted on patients with cutaneous diseases have reported the presence of both HPyV6- and 7-DNA in tissue specimens. Three dyskeratotic dermatosis cases tissue revealed 2 positives for HPyV6 and 1 positive for $\mathrm{HPyV} 7$, and in previously 12 $\mathrm{HPV}$-induced warts tested showed that 3 positives for HPyV6 and 8 for HPyV7 50,53. In addition, HPyV7-DNA was found in all three biopsies of pruritic patients, thus the diagnostic term HPyV 7-associated pruritic rash has been introduced in transplantation medicine ${ }^{51,52}$. The higher prevalence of both viruses in skin disease samples could be attributed to their high occurrence in the skin of healthy individuals. However, the viral load and prevalence of both viruses seem to increase in the skin of elderly people, in particular immunosuppressed individuals, which may lead to associated skin disorders ${ }^{65}$.

The prevalence of HPyV6- and 7-DNA in non-neoplastic non-cutaneous diseases appears to be much lower compared to non-neoplastic skin diseases. Our group reported the presence of HPyV7-DNA in $(3 / 8 ; 40 \%)$ of non-malignant thymic hyperplasia ${ }^{31}$. Other studies detected HPyV6-DNA in (12/226; 5.3\%) of tonsillitis tissue specimens Table $256,57,58$. The detection rate of both viruses was found to be slightly higher in tissue specimens in contrast to body fluids specimens ${ }^{24,31,50,51,52,53,54,56,57,58,59,60,61,63,64}$. For instance, in cerebrospinal fluid, HPyV6-DNA was detected in (1/14; 7.1\%) samples from HIV patients with leukoencephalopathies and $(1 / 135 ; 0.7 \%)$ samples from patients with meningoencephalitis ${ }^{61,62}$. In addition, $(5 / 91 ; 5.5 \%)$ bile fluid samples from acute gallstone cholangitis patients tested positive for $\mathrm{HPyV} 6{ }^{64}$. In comparison, there was a low detection rate $(<2 \%)$ of both viruses in nasopharyngeal aspirates specimens collected from respiratory symptomatic patients ${ }^{59,60}$. Similarly, the presence of HPyV6 and 7 was uncommon $(<1 \%)$ in the feces obtained from patients with gastrointestinal illness for routine lab investigation ${ }^{59}$. Overall, the prevalence of HPyV6 and HPyV7 in formalinfixed-paraffin-embedded (FFPE) and fresh frozen biopsies were elevated compared to liquid biopsy specimens, in which the prevalence of HPyV6 and HPyV7 was 1.1 and $0.2 \%$, respectively Table 2 . 
Table 2 Prevalence of HPyV6 and HPyV7 in human non-neoplastic diseases

\begin{tabular}{|c|c|c|c|c|c|c|}
\hline Non-neoplastic disease & Specimen & $\begin{array}{l}\text { Samples } \\
\text { n }\end{array}$ & Method & $\begin{array}{l}\text { HPyV6 } \\
\text { Positive n } \\
(\%) \\
\end{array}$ & $\begin{array}{l}\text { HPyV7 } \\
\text { Positive n } \\
(\%)\end{array}$ & Reference \\
\hline \multicolumn{7}{|l|}{ Cutaneous diseases } \\
\hline Dyskeratosis and parakeratosis & FFPE & 3 & $\begin{array}{l}\mathrm{PCR} / \mathrm{qPCR} / \mathrm{IHC} / \\
\text { Seq. }\end{array}$ & $2(66.6)$ & $1(33.3)$ & {$[50]$} \\
\hline Lichen simplex chronicus & FF & 13 & rt-PCR & $1(7.7)$ & ND & {$[24]$} \\
\hline $\begin{array}{l}\text { Pruritic in Cardiac transplant } \\
\text { recipient }\end{array}$ & FFPE & 1 & IHC/PCR & NA & $1(100)$ & {$[51]$} \\
\hline Pruritic Rash (lung transplant) & FFPE & 2 & $\begin{array}{l}\text { IHC/PCR/RCA } \\
\text { PCR/rt-PCR }\end{array}$ & NA & $2(100)$ & {$[52]$} \\
\hline Viral warts & FFPE & 12 & qPCR & $3(25)$ & $8(66.7)$ & {$[53]$} \\
\hline $\begin{array}{l}\text { Angiolymphoid hyperplasia } \\
\text { with eosinophilia or Kimura } \\
\text { disease }^{\text {a }}\end{array}$ & $\mathrm{FF}$ & 1 & $\begin{array}{l}\text { Shotgun } \\
\text { metagenomicl } \\
\text { FISH }\end{array}$ & $1(100)$ & NA & {$[54]$} \\
\hline $\begin{array}{l}\text { Angiolymphoid hyperplasia } \\
\text { with eosinophilia }\end{array}$ & FFPE & 5 & qPCR/IHC & $4(80)$ & 0 & [55] \\
\hline Kimura disease & FFPE & 5 & $\mathrm{qPCR} / \mathrm{IHC}$ & $4(80)$ & $1(20)$ & {$[55]$} \\
\hline Kikuchi disease & FFPE & 38 & qPCR & $3(8)$ & 0 & {$[55]$} \\
\hline Dermatopathic lymphadenitis & FFPE & 28 & qPCR & $1(4)$ & 0 & {$[55]$} \\
\hline \multicolumn{7}{|l|}{ Non-cutaneous diseases } \\
\hline Thymic hyperplasia & FFPE & 20 & PCR/IHC/FISH & NA & $8(40)$ & {$[31]$} \\
\hline $\begin{array}{l}\text { Chronic tonsillitis \& tonsillar } \\
\text { hypertrophy }\end{array}$ & $\mathrm{FF}$ & 78 & Luminex-assay & $6(7.7)$ & ND & {$[56]$} \\
\hline $\begin{array}{l}\text { Chronic tonsillitis \& tonsillar } \\
\text { hyperplasia }\end{array}$ & FF & 40 & qPCR & $1(2.5)$ & ND & {$[57]$} \\
\hline $\begin{array}{l}\text { Tonsillectomy (due to chronic } \\
\text { tonsillitis, peritonsillar abscess, } \\
\text { or sleep apnea syndrome) }\end{array}$ & $\begin{array}{l}\text { FF and } \\
\text { FFPE }\end{array}$ & 108 & qPCR & $5(4.6)$ & $1(0.9)$ & {$[58]$} \\
\hline $\begin{array}{l}\text { Patients with respiratory } \\
\text { symptoms }\end{array}$ & NPA & 1232 & rt-PCR & $2(0.16)$ & $3(0.24)$ & {$[59]$} \\
\hline Respiratory tract infections & NPA & 887 & rt-PCR & $15(1.7)$ & ND & {$[60]$} \\
\hline $\begin{array}{l}\text { HIV patients with } \\
\text { leukoencephalopathies. }\end{array}$ & CSF & 14 & NGS/PCR & $1(7.1)$ & NA & {$[61]$} \\
\hline Meningoencephalitis & CSF & 135 & qPCR & $1(0.74)$ & 0 & {$[62]$} \\
\hline $\begin{array}{l}\text { Immunocompromised patients } \\
\text { scheduled to receive transplants }\end{array}$ & $\begin{array}{l}\text { NPS, urine, } \\
\text { fecal, plasma }\end{array}$ & 32 & rt-PCR & $3(9.4)$ & $1(3.1)$ & {$[63]$} \\
\hline Acute gallstone cholangitis & Bile fluids & 91 & rt-PCR & $5(5.5)$ & NA & {$[64]$} \\
\hline $\begin{array}{l}\text { Patients with gastrointestinal } \\
\text { illness }\end{array}$ & Fecal & 185 & rt-PCR & $1(0.5)$ & $1(0.5)$ & [59] \\
\hline
\end{tabular}

aAHE case with Kimura disease clinical syndromes

$F F$ fresh frozen tissue, FFPE formalin-fixed paraffin-embedded, FISH fluorescence in situ hybridization, $I H C$ immunohistochemistry, $N A$ not applicable, $N D$ not detected, $N G S$ next-generation sequencing, $N P A$ nasopharyngeal aspirates, NPS nasopharyngeal swab, $P C R$ polymerase chain reaction, $q P C R$ quantitative polymerase chain reaction, $r t-P C R$ real-time polymerase chain reaction, Seq Sanger sequencing 


\section{HPyV6 and HPyV7 DNA prevalence in primary cutaneous malignancies}

According to the World Health Organization, the incidence of skin tumors has increased over the past decades, approximately 8500 new cases of skin cancers are reported daily in the United States ${ }^{66,67}$. Up to date, the presence of the DNA of five HPyVs has been reported in the human skin: $\mathrm{MCPyV}, \mathrm{HPyV} 6, \mathrm{HPyV} 7, \mathrm{HPyV}$, and trichodysplasia spinulosa-associated polyomavirus ${ }^{40}$. Of these, only MCPyV has been identified as a novel human tumor virus closely linked to the etiopathogenesis of the majority of MCCs. Since HPyV6 and 7 were isolated from the skin their possible contribution to the etiopathogenesis of skin cancers has been studied intensively ${ }^{24,25,26,27,28,29,30,68}$. Numerous studies, summarized in Table 3, have assessed the prevalence of HPyV6- and 7-DNA in primary cutaneous malignancies, including epithelial, neuroendocrine, and lymphoid skin cancers.

One study reported the presence of HPyV6- and HPyV7-DNA (2/47; 4.3\%) in malignant melanoma (MM) specimens using rt-PCR ${ }^{25}$. In non-melanoma skin cancer tissues, both HPyV6- and 7-DNA have also been identified. Four studies have shown the presence of HPyV6-DNA in the most common human skin cancer, i.e. basal cell carcinoma (BCC) Table 3 . The detection rate was between 2 to $21 \%$, and one study confirmed the detection of HPyV6 in BCC by FISH 25, 27, 29, 68. HPyV7-DNA was identified in 4\% of BCC cases by rt-PCR in a single study ${ }^{25}$. Studies on squamous cell carcinoma (SCC), which is the second most common human cutaneous malignancy, revealed a prevalence between 3 to 38\% for HPyV6 25, 27,28,29, 68 . Other studies tested keratoacanthoma and trichoblastoma for HPyV6-DNA, although no studies have tested trichoblastoma for HPyV7 27, 68 . HPyV6- and 7-DNA were detected in $<2 \%$ of tissue biopsies from MCC and $<4 \%$ of actinic keratosis tissues $27,29,30,53,69$. To date, only one study has analyzed verrucous keratosis tissues, and (3/4; 75\%) specimens were positive for both viruses ${ }^{53}$. Another study by Schrama et al. detected HPyV6 in all tested 18 epithelial proliferation specimens (SCC and/or keratoacanthoma obtained from MM patients treated with the BRAF inhibitor vemurafenib) and $\mathrm{HPyV7}$ in 17/18 specimens using rt-PCR combined with immunohistochemistry (IHC) using 6V32 and 6V12 antibodies targeting HPyV6 $\mathrm{VP} 1$ and have cross-reactivity with $\mathrm{HPyV} 7{ }^{26}$. It is of interest to mention that only very few studies report the usage of IHC to screen for HPyV6 and 7 protein expression, due to the lack of commercially available HPyV6- or 7-specific antibodies. In this respect, one may also consider the use of the commonly PAb416 antibody used in routine laboratories originally directed against the LTAg of SV40. PAb416 antibody which detects conserved epitope region of diverse $\mathrm{HPyVs}$ including $\mathrm{HPyV} 6$ and 7 but not detecting MCPyV which there is a commercial antibody (CM2B4) available to detect its $\operatorname{LTAg}^{72,73}$.

Additionally, primary cutaneous lymphomas have been tested for the presence of HPyV6and 7-DNA: cutaneous T-cell lymphoma (CTCL) patients, and all three studies showed 
Table 3 Prevalence of HPyV6 and HPyV7 in primary cutaneous malignancies

\begin{tabular}{|c|c|c|c|c|c|c|}
\hline Cancer & $\begin{array}{l}\text { Specimen } \\
\text { types }\end{array}$ & Samples n & Method & $\begin{array}{l}\text { HPyV6 Positive } \\
\text { n (\%) }\end{array}$ & $\begin{array}{l}\text { HPyV7 Positive } \\
\text { n (\%) }\end{array}$ & Reference \\
\hline MM & FFPE & 47 & rt-PCR/Seq. & $2(4.3)$ & $2(4.3)$ & {$[25]$} \\
\hline AK & FFPE & 31 & qPCR & $1(3)$ & ND & [27] \\
\hline AK & FFPE & 92 & Seq. & $4(4.3)$ & NA & [69] \\
\hline AK & FFPE & 2 & qPCR & $\mathrm{ND}$ & $1(50)$ & {$[53]$} \\
\hline VK & FFPE & 4 & $\mathrm{qPCR}$ & $3(75)$ & $3(75)$ & {$[53]$} \\
\hline BCC & FFPE & 18 & qPCR & $10(5.5)$ & $\mathrm{ND}$ & [29] \\
\hline BCC & FFPE & 41 & qPCR & $3(7)$ & ND & {$[27]$} \\
\hline BCC & FFPE & 50 & rt-PCR/Seq. & $1(2)$ & $2(4)$ & {$[25]$} \\
\hline BCC & FFPE & 109 & PCR/Seq./FISH & $23(21.1)$ & NA & {$[68]$} \\
\hline SCC & FFPE & 8 & qPCR & $1(12)$ & ND & {$[27]$} \\
\hline SCC & FFPE & 21 & qPCR & $8(38)$ & ND & [29] \\
\hline SCC & FFPE & 52 & qPCR & $2(4)$ & ND & {$[27]$} \\
\hline SCC & FFPE & 86 & PCR/Seq./FISH & $8(9.3)$ & NA & {$[68]$} \\
\hline SCC & FFPE & 63 & rt-PCR/Seq. & $2(3.2)$ & $1(1.6)$ & {$[25]$} \\
\hline $\begin{array}{l}\text { SCC IN CLL cases } \\
\text { who had BMT }\end{array}$ & FFPE & 3 & $\begin{array}{l}\text { Bead-based } \\
\text { multiplex PCR }\end{array}$ & $1(33.3)$ & ND & {$[28]$} \\
\hline SCC & FFPE & 11 & qPCR & $4(36.4)$ & $6(54.5)$ & {$[53]$} \\
\hline SCC & FFPE & $\begin{array}{l}17(6 \\
\text { patients) }\end{array}$ & rt-PCR/IHC & $17(100)$ & $17(100)$ & {$[26]$} \\
\hline KA & FFPE & 42 & qPCR & $2(5)$ & ND & {$[27]$} \\
\hline KA & FFPE & 59 & $\mathrm{PCR} / \mathrm{Seq} / \mathrm{FISH}$ & $25(42.3)$ & NA & {$[68]$} \\
\hline TB & FFPE & 45 & PCR/Seq & $10(22.2)$ & NA & {$[68]$} \\
\hline MCC & FFPE & 20 & qPCR & $2(10)$ & ND & {$[29]$} \\
\hline MCC & FFPE & 28 & rt-PCR & $1(3.5)$ & $1(3.5)$ & {$[30]$} \\
\hline CTCLs & FFPE & 116 & rt-PCR & $6(5.20)$ & $1(0.90)$ & {$[70]$} \\
\hline CTCLs & $\mathrm{FF}$ & 71 & rt-PCR & $13(18.3)$ & ND & {$[24]$} \\
\hline CTCLs & FF and FFPE & 35 & rt-PCR & $7(20)$ & $3(8.6)$ & {$[71]$} \\
\hline Total & & 1072 & & $157 / 1072(14.6)$ & $37 / 681(5.4)$ & \\
\hline
\end{tabular}

$A K$ actinic keratosis, $B C C$ basal cell carcinoma, $B M T$ bone marrow transplantation, $C L L$ chronic lymphocytic leukemia, CTCLs cutaneous T-cell lymphomas, FFPE formalin-fixed paraffin-embedded, FISH fluorescence in situ hybridization, $I H C$ immunohistochemistry, $F F$ fresh frozen tissue, $K A$ keratoacanthoma, $M C C$ Merkel cell carcinoma, $M M$ malignant melanoma, $N A$ not applicable, $N D$ not detected, $P C R$ polymerase chain reaction, $q P C R$ quantitative polymerase chain reaction, $r t-P C R$ real-time polymerase chain reaction, SCC squamous cell carcinoma, Seq Sanger sequencing, TB trichoblastoma, $V K$ verrucous keratosis

that CTCLs were positive (5.20 to 20\%) for HPyV6- and (0.90 to 8.6\%) for HPyV7DNA, while no cutaneous B-cell lymphoma was positive for either virus ${ }^{24,70,71}$. Overall, results showed that the occurrence of HPyV6-DNA in CTCL was more frequent than HPyV7-DNA Table 3.

It is remarkable that HPyV6-DNA is far more often detected than HPyV7-DNA in human neoplastic skin diseases (Fig. 2), which may indicate a role for HPyV6 in the etiopathogenesis of these cancers. However, the reported copy loads of HPyV6- and 7-DNA in diverse skin cancers do not clearly support this hypothesis. Studies reported a generally low viral load of HPyV6- and 7-DNA-positive skin cancers which does not reflect the situation of high viral load of MCPyV in MCC 25, 27, 29, 53, 74 . Nevertheless, 
viral load copies of HPyV6 per cell range (0.00014-0.14) and MCPyV (0.0016-0.36) were almost in the same range in non-MCC tumors such as SCC, BCC, and MM, while HPyV7 copy numbers (0.000079-0.0094) were much lower. Moreover, specimens from healthy skin specimens showed the same range of HPyV6-DNA and MCPyV-DNA viral load as non-MCC samples 25, 27, 29, 53. Notably, HPyV7-DNA was more often detectable than HPyV6-DNA in non-cutaneous cancers, while HPyV6-DNA was identified more often in skin malignancies Tables 2 and 3 .

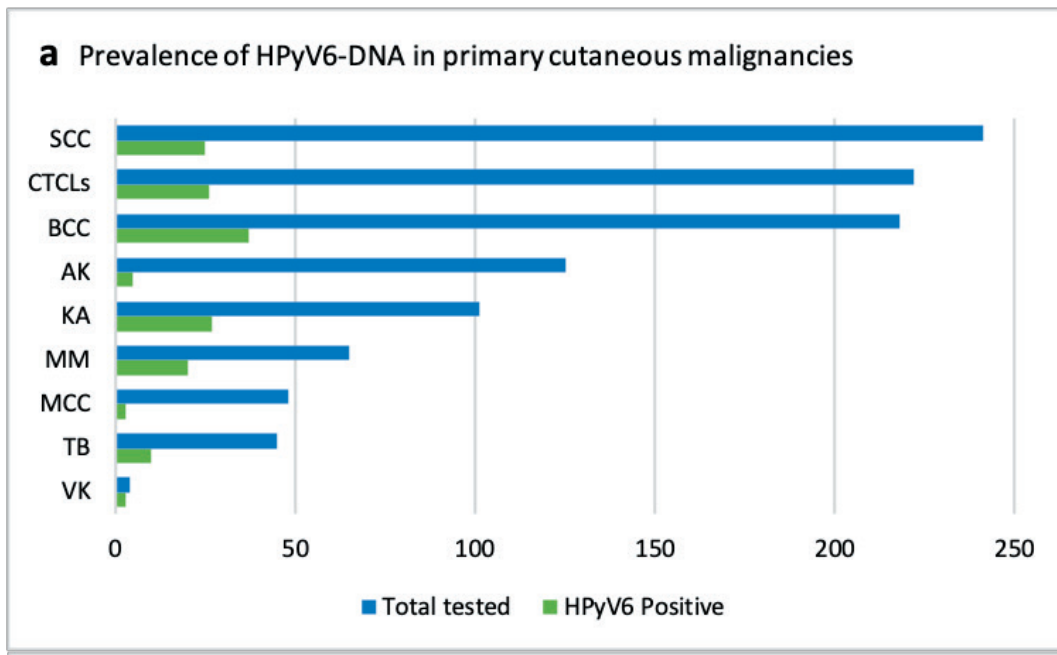

\section{b Prevalence of HPyV6-DNA in primary cutaneous malignancies}

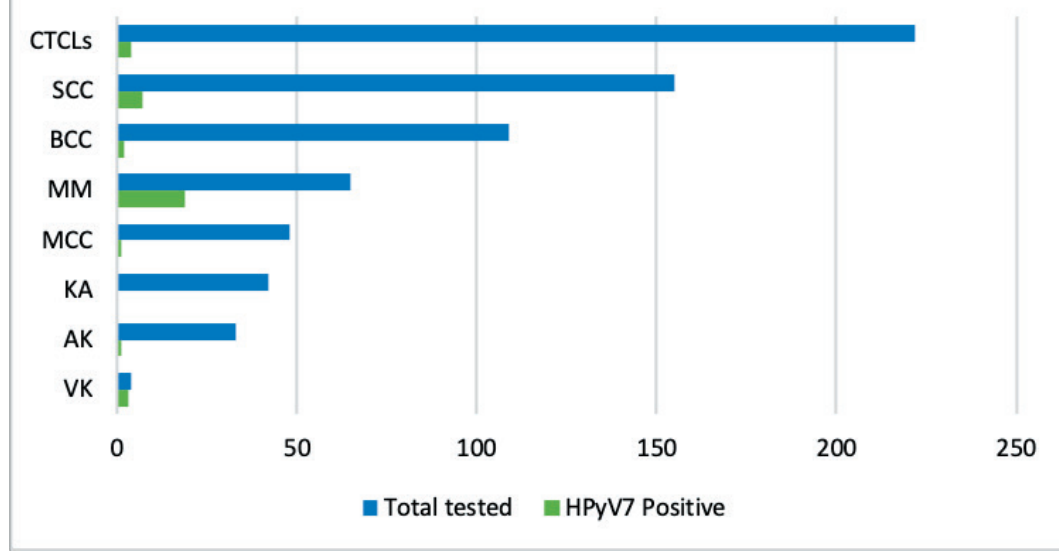

Fig. 2 Representing a summary of the prevalence of HPyV6-DNA (a) and HPyV7-DNA (b) in tissues of cutaneous malignancies. AK, actinic keratosis; BCC, basal cell carcinoma; CTCLs, cutaneous T-cell lymphomas; KA, keratoacanthoma; MCC, Merkel cell carcinoma; MM, malignant melanoma; SCC, squamous cell carcinoma; $\mathrm{TB}$, trichoblastoma; $\mathrm{VK}$, verrucous keratosis 


\section{HPyV6 and HPyV7 in non-cutaneous malignancies}

The prevalence of HPyV6 and 7 have also been studied in a variety of non-cutaneous malignancies summarized in Table 4. In tissue specimens, HPyV7- but not HPyV6-DNA was frequently $(62 \%)$ found in thymic epithelial tumors ${ }^{31}$. In contrast, HPyV6-DNA was more prevalent $(5.4 \%)$ in tonsillar SCC ${ }^{57,58}$.

Table 4 Prevalence of HPyV6 and HPyV7 in non-cutaneous other human malignancies

\begin{tabular}{|c|c|c|c|c|c|c|}
\hline Tumor & Specimen & $\begin{array}{l}\text { Samples } \\
\text { n }\end{array}$ & Method & $\begin{array}{l}\text { HPyV6 Positive } \\
\text { n (\%) }\end{array}$ & $\begin{array}{l}\text { HPyV7 Positive } \\
\text { n (\%) }\end{array}$ & Reference \\
\hline BC & FF & 54 & rt-PCR & $1(2)$ & $1(2)$ & {$[75]$} \\
\hline LSCC & $\mathrm{FF}$ & 7 & $\begin{array}{l}\text { Luminex platform (Multiplex } \\
\text { PCR) }\end{array}$ & $1(14.3)$ & ND & {$[76]$} \\
\hline TETs & FFPE & 37 & FISH/PCR/Seq/IHC & ND & $23(62.2)$ & {$[31]$} \\
\hline TSCC & FF & 38 & qPCR & $2(5.3)$ & $1(2.6)$ & [57] \\
\hline TC & $\begin{array}{l}\text { FF and } \\
\text { FFPE }\end{array}$ & 112 & qPCR & $6(5.4)$ & $2(1.8)$ & {$[58]$} \\
\hline CCA & FFPE & 42 & PCR/FISH/RISH/IHC & $4(9.5)$ & $19(45.2)$ & {$[77]$} \\
\hline
\end{tabular}

$B C$ breast cancer, $C C A$ cholangiocarcinoma, FFPE formalin-fixed paraffin-embedded, $F F$ Freshly frozen tissue, FISH fluorescence in situ hybridization, $L S C C$ laryngeal squamous cell carcinoma, $N D$ not detected, $P C R$ polymerase chain reaction, $q P C R$ quantitative polymerase chain reaction, $r t-P C R$ real-time polymerase chain reaction, RISH RNA fluorescence in situ hybridization, Seq Sanger sequencing, $T C$ tonsillar carcinoma, TETs thymic epithelial tumors, TSCC tonsillar squamous cell carcinoma

In body fluids specimens, Chan et al. investigated the presence of HPyVs in the bile fluid of patients with a variety of hepatobiliary malignancies ${ }^{64}$. HPyV6 but not HPyV7 was detected in 27.6, 10.7, 16.7, and $20 \%$ of bile specimens from cholangiocarcinoma (CCA), hepatocellular carcinoma, pancreatic carcinoma, and gallbladder carcinoma patients, respectively ${ }^{64}$. We recently identified the presence of HPyV6- and 7-DNA in cholangiocarcinoma cases at the single-cell level by diverse molecular biology techniques for the detection of viral DNA, mRNA, and protein expression ${ }^{77}$. We found that HPyV7 was more prevalent than HPyV6 and indeed the presence of these viruses was not restricted to CCA tissues but also found in adjacent microscopically non-neoplastic hepatocytes ${ }^{77}$.

Overall, among patients with different non-cutaneous cancers, HPyV7-DNA was more frequently detected than HPyV6-DNA in tissues. Yet, it remains unclear if and how HPyV7 might contribute to the tumorigenesis of these thymic epithelial tumors or cholangiocarcinomas, or that it rather is present in the context of viral latency.

\section{Latency and tropism}

The frequent prevalence of HPyV6- and 7-DNA in non-neoplastic tissues along with their high seropositivity indicates that these viruses remain in long-term latency in humans. Therefore, it is important to unmask the host cells facilitating this long-term latency in 
healthy individuals to understand the host cell-type specific encoded proteins needed for viral entrance, replication, and transcription.

So far, the underlying mechanisms of tissue tropism or the possible latency of HPyV6 and 7 are still unknown. Other HPyVs have been reported to remain in latency within the epithelium of the proximal tubule of the kidney (BKPyV) while JCPyV is found in the brain ${ }^{3}$. In other studies, $\mathrm{BKPyV}$ and JCPyV were suggested to be found latent in lymphoid tissues and lymphocytes ${ }^{78}$. Since the seroprevalence of HPyV6 and 7 is high in the general population, it is difficult to ultimately determine the latency compartment of these two HPyVs based on the currently available literature. However, keratinocytes have been identified to be the potential primary target of $\mathrm{HPyV7}$ infection and replication ${ }^{50}$. In addition, since HPyV 6 and 7 have been detected in BCC, further studies are needed to investigate whether they act similar to HPV by infecting the basal epithelial cells through lesions in the epidermis and undergo episomal replication in the parabasal layers $25,27,29,68,79$.

\section{Direct versus indirect HPyVs-related carcinogenesis}

The potential contribution of oncogenic viruses is either direct and/or indirect in the carcinogenesis of virus-associated tumors ${ }^{80,81}$. MCPyV is the only HPyV found to be clonally integrated into the genome of human cancer, namely MCC. Next to its clonal integration, the $\mathrm{MCPyV}$ genome carries tumor-specific mutations within the large tumor antigen (LTAg) introducing stop codons and thus abrogating the helicase expression and rendering MCPyV replication-deficient ${ }^{15,17}$. In direct carcinogenesis, one would expect a high expression of viral proteins in every tumor cell, which indeed is seen for the MCPyV-LTAg protein in MCPyV positive MCC ${ }^{15,17,82}$. In particular, the binding of truncated LTAg to the retinoblastoma protein-binding (RB) maintains the replication of MCPyV-positive MCC cells ${ }^{3,26,83}$. During the preparation of this review it occurred, that only a few studies report the protein expression of HPyV6 and 7 in neoplastic cells, possibly indicating such a direct tumorigenic role as shown for MCPyV and MCC. However, HPyV6- and 7-LTAg expression are not found in all tumor cells in the above mentioned possibly HPyV6 and 7-associated human cancers. Thus, it seems unlikely that both HPyVs play an important direct contribution to human tumorigenesis ${ }^{26,31,77}$.

Human tumor viruses also can induce neoplasms by indirect mechanisms via the interaction between viral antigens, environmental factors, and other modifications to the host genome ${ }^{80}$. Inflammatory reactions due to the immune response to viral infection exposure can become eradicated and induce acute inflammation which may also result in chronic inflammation ${ }^{84,85}$. Reactive oxygen radicals can be produced years later following chronic inflammation and trigger modifications in the host nucleic acids and then inducing transformation as it is known for hepatitis $B$ and $C$ viruses ${ }^{84,86}$. 
For instance, the hepatitis $\mathrm{C}$ virus is not detected in the cancer cells that may develop after many years of infection but are found in the adjacent lesions to the hepatocellular carcinoma ${ }^{87,88,89,90}$. The infection by the virus alone is not sufficient to develop a tumor, and persistent viral infection precedes possibly many years before a cancer develops by an indirect mechanism such as chronic inflammation. The recent finding of HPyV6 and 7 in hepato- and cholangiocellular tissues possibly indicates such a physiopathologic mechanism of chronic inflammation-related indirect carcinogenesis also for HPyV6 and $7{ }^{77}$. However, more studies are needed to further elucidate and establish chronic inflammation-related indirect carcinogenesis for HPyV6 and 7.

Besides the direct and indirect HPyVs-related carcinogenesis, the "hit and run" hypothesis has been proposed to explain a possible role of viral agents to mediate cellular transformation ${ }^{91}$. The "hit and run" concept was proposed by Skinner in 1976 as a model where the virus is required to initiate the tumorigenesis process by leading to mutations, which maintain the tumorigenesis resulting in tumor with loss of the viral genes in the later stages ${ }^{91}$. Few studies have suggested the "hit and run" mechanism to mediate transformation by $\mathrm{BKPyV}$, JCPyV, and MCPyV ${ }^{92,93,94,95,96}$. However, this hypothesis remains speculative until further evidence for "hit and run" for-HPyV associated diseases is established.

In addition, HPyVs may contribute to human tumorigenesis together with other oncogenic viruses assuming, that two or maybe more different viruses potentially infect the same cells and interact with each other in the etiopathogenesis of human cancers 97,98,99,100. Primary evidence for interplay and helper functions of viruses had been established since long, especially for adenoviruses and herpesviruses ${ }^{101,102}$. Of interest, Heilbronn et al. reported the finding that cytomegalovirus (CMV) infection can trigger JCPyV-DNA replication in cultured human fibroblasts ${ }^{103}$. Indirect potential evidence for possible interplay and helper functions is currently restricted to observational findings: $\mathrm{BKPyV}$ has been found to be associated with the induction of cervical intraepithelial neoplasias, particularly in immunosuppressed patients ${ }^{104,105}$. Although these findings might point to an interaction of certain high-risk HPV types with BKPyV, they are by far no causal proof that $\mathrm{BKPyV}$ contributes to the HPV-related etiopathogenesis of cervical cancer. Coinfection with different human viruses has been also reported in oropharyngeal and oral cavity cancers ${ }^{100}$. Although there are yet no functional data available concerning possible interplay and helper functions of $\mathrm{HPyVs}$ with other viruses in human cancers, it might be interesting to study this for HPyV6 and 7. 


\section{Further studies to elucidate the role of HPyV6 and 7 in etiopathogenesis of human cancers}

The HPyV6 and 7 contributions to human tumorigenesis are not yet elucidated. Therefore, several important questions regarding the pathogenesis of both viruses need to be addressed. For example, how to confirm the interaction of HPyV6 and HPyV7 in the inactivation of $\mathrm{p} 53$ and retinoblastoma protein $(\mathrm{pRb})$, or in the transformation function. Rozenblatt-Rosen et al. namely proposed this mechanism in which HPyV6 and 7 both LTAg and sTAg proteins to bind p53 and inactivate it leading to tumorigenesis 32. MCPyV is the only known HPyV that is found to be clonally integrated into MCC cases, in particular, the binding of truncated LTAg to the $\mathrm{pRb}$ was found to maintain the growth of MCC in MCPyV-positive cell lines ${ }^{3,83,106}$. Furthermore, SV40, which is a non-HPyV, was reported to bind $\mathrm{p} 53$ and $\mathrm{Rb}$ and inactivate their function ${ }^{107,108,109}$. In addition, one study has shown that HPyV6 sTAg is capable to inactivate the tumor suppressor protein phosphatase $2 \mathrm{~A}(\mathrm{PP} 2 \mathrm{~A})$ that results in hyperphosphorylation of the MEK-ERK pathway and leads to abnormal proliferation ${ }^{110,111}$.

The presence of HPyV6- and 7-DNA and their association with human malignancies have commonly been tested by PCR and qPCR. However, the conclusions that can be drawn from these studies restricting their level of evidence to the proof of viral nucleic acids in homogenized tissue extracts are unfortunately very limited. The meaning of the presence of viral nucleic acids must remain inconclusive because these findings cannot be interpreted on the single-cell level in the histomorphological context of the specific disease. In addition, only limited reliable data are available on the viral protein expression, because the detection of antibodies by IHC in FFPE tissues are lacking. A possible screening approach might start with the use of the PAb416 antibody followed by specific HPyV6 and 7 PCR. In addition, studies definitely are needed in order to investigate next to the presence of HPyV6-DNA and 7-DNA, if these viruses are integrated into the tumor genome and possibly carry tumor-specific mutations within the viral helicase of LTAg in analogy to MCPyV ${ }^{15}, 17$. This will e.g. also help to understand the role of HPyV6 and 7 in skin carcinogenesis as a possible co-factor of UV-radiation as it is discussed for HPV5 and 8 association skin carcinogenesis ${ }^{49,112}$. 


\title{
Conclusion
}

In this review, we aimed to comprehensively analyze the literature for a possible role of the two closely related HPyV6 and 7 in human diseases and cancers. The seropositivity of HPyV6 was found to be higher than HPyV7 and both increased with age. The etiopathogenetical contribution of these viruses to human cancers remains unclear. Studies revealed that low HPyV6 and HPyV7 prevalence in non-neoplastic tumors resembled those in malignancies. Interestingly, $\mathrm{HPyV} 6$ prevalence was higher in skin malignancies than that of $\mathrm{HPyV} 7$. In contrast, $\mathrm{HPyV} 7$ was more frequently detected in non-cutaneous malignancies compared to HPyV6.

Based on sero- and tissue-prevalence $\mathrm{HPyV} 6$ and 7 remain important putative candidates for contributing to the etiopathogenesis of human disease, including skin cancers. There is an urgent need to further define their presence within cutaneous lesions e.g., keratoacanthoma, squamous cell carcinoma, and basal cell carcinoma, and to apply additional molecular techniques to unravel the molecular basis of virus-host interactions and assess a possible role of viral integration or mutation of the LTAg in skin cancer development. The same applies for HPyV7-associated tumors e.g., cholangiocellular carcinomas or thymomas. Further studies are needed to understand the contribution of HPyV6 and HPyV7 to human cancers.

\begin{abstract}
Abbreviations:
HPyVs: Human polyomaviruses; HPyV6: Human polyomavirus 6; HPyV7: Human polyomavirus 7; PyV's: Polyomaviruses; MCPyV: Merkel cell polyomavirus; MCC: Merkel cell carcinoma; VP1: Viral protein 1; VLP: Virus-like particles; ELISA: Enzyme-linked immunosorbent assays; GST: glutathione S-transferase; HPV: human papillomaviruses; ALHE: Angiolymphoid hyperplasia with eosinophilia ; FISH: Fluorescence in situ hybridization; FFPE: Formalin-fixed-paraffin-embedded; MM: Malignant melanomas; BCC: Basal cell carcinoma; SCC: Squamous cell carcinoma; IHC: Immunohistochemistry; CTCL: Cutaneous T-cell lymphomas; CCA: cholangiocarcinoma; LTAg: Large tumor antigen.
\end{abstract}

\section{Acknowledgments:}

None to declare.

\section{Funding:}

None to declare.

\section{Availability of data and materials:}

Not applicable. 


\section{Ethics approval and consent to participate:}

Not applicable.

\section{Consent for publication:}

Not applicable.

\section{Competing interests:}

The authors declare that they have no competing interests.

\section{Author Contributions}

FK and AzH conceived and designed the study. FK, GM, and DL searched the literature, extract and analyzed the data. FK and AzH wrote the manuscript. AK, VW, RA, EJS were contributed to writing specific parts of the manuscript. AzH is the principal investigator and set up and supervised this review. All the authors read and approved this manuscript for publication.

\section{Author details}

${ }^{1}$ Department of Pathology, GROW-School for Oncology \& Developmental Biology, Maastricht University, Medical Centre+, Maastricht, the Netherlands. ${ }^{2}$ Department of Laboratory Medicine, Faculty of Applied Medical Sciences, Albaha University, Albaha, Saudi Arabia. ${ }^{3}$ Department of Medical Laboratories Technology, Faculty of Applied Medical Sciences, Jazan University, Jazan, Saudi Arabia. ${ }^{4}$ Department of Hematology, The Affiliated Hospital of Southwest Medical University, Luzhou, China. ${ }^{5}$ Department of Internal Medicine IV, RWTH Aachen University Hospital, Aachen, Germany 


\section{REFERENCES}

1. Johne R, Buck CB, Allander T, Atwood WJ, Garcea RL, Imperiale MJ, et al. Taxonomical developments in the family Polyomaviridae. Archives of virology. 2011;156(9):1627-34.

2. Johne R, Müller H. Polyomaviruses of birds: etiologic agents of inflammatory diseases in a tumor virus family. Journal of virology. 2007;81(21):11554-9.

3. DeCaprio JA, Garcea RL. A cornucopia of human polyomaviruses. Nat Rev Microbiol. 2013;11(4):26476.

4. Small JA, Khoury G, Jay G, Howley PM, Scangos GA. Early regions of JC virus and BK virus induce distinct and tissue-specific tumors in transgenic mice. Proceedings of the National Academy of Sciences of the United States of America. 1986;83(21):8288-92.

5. Dalrymple SA, Beemon KL. BK virus $T$ antigens induce kidney carcinomas and thymoproliferative disorders in transgenic mice. Journal of virology. 1990;64(3):1182-91.

6. Ramqvist T, Dalianis T. Murine polyomavirus tumour specific transplantation antigens and viral persistence in relation to the immune response, and tumour development. Seminars in cancer biology. 2009;19(4):236-43.

7. zur Hausen $\mathrm{H}$. Novel human polyomaviruses-re-emergence of a well known virus family as possible human carcinogens. International journal of cancer. 2008;123(2):247-50.

8. Moens U, Prezioso C, Pietropaolo V. Genetic Diversity of the Noncoding Control Region of the Novel Human Polyomaviruses. Viruses. 2020;12(12).

9. ICTV-Report-pConsortium. Report on the taxonomy of the Polyomaviridae. Virus Taxonomy: 2018b Release. International Committee on Taxonomy of Viruses. 2019.

10. Gedvilaite A, Tryland M, Ulrich RG, Schneider J, Kurmauskaite V, Moens U, et al. Novel polyomaviruses in shrews (Soricidae) with close similarity to human polyomavirus 12 . The Journal of general virology. 2017;98(12):3060-7.

11. Fahsbender E, Altan E, Estrada M, Seguin MA, Young P, Leutenegger CM, et al. Lyon-IARC Polyomavirus DNA in Feces of Diarrheic Cats. Microbiol Resour Announc. 2019;8(29).

12. Ondov BD, Starrett GJ, Sappington A, Kostic A, Koren S, Buck CB, et al. Mash Screen: highthroughput sequence containment estimation for genome discovery. Genome biology. 2019;20(1):232.

13. Buck CB, Van Doorslaer K, Peretti A, Geoghegan EM, Tisza MJ, An P, et al. The Ancient Evolutionary History of Polyomaviruses. PLoS pathogens. 2016;12(4):e1005574.

14. Torres C. Evolution and molecular epidemiology of polyomaviruses. Infect Genet Evol. 2020;79:104150.

15. Feng H, Shuda M, Chang Y, Moore PS. Clonal integration of a polyomavirus in human Merkel cell carcinoma. Science (New York, NY). 2008;319(5866):1096-100.

16. Kassem A, Schopflin A, Diaz C, Weyers W, Stickeler E, Werner M, et al. Frequent detection of Merkel cell polyomavirus in human Merkel cell carcinomas and identification of a unique deletion in the VP1 gene. Cancer research. 2008;68(13):5009-13. 
17. Shuda M, Feng H, Kwun HJ, Rosen ST, Gjoerup O, Moore PS, et al. T antigen mutations are a human tumor-specific signature for Merkel cell polyomavirus. Proceedings of the National Academy of Sciences of the United States of America. 2008;105(42):16272-7.

18. Bouvard V, Baan RA, Grosse Y, Lauby-Secretan B, El Ghissassi F, Benbrahim-Tallaa L, et al. Carcinogenicity of malaria and of some polyomaviruses. The Lancet Oncology. 2012;13(4):339-40.

19. Pantulu ND, Pallasch CP, Kurz AK, Kassem A, Frenzel L, Sodenkamp S, et al. Detection of a novel truncating Merkel cell polyomavirus large $\mathrm{T}$ antigen deletion in chronic lymphocytic leukemia cells. Blood. 2010;116(24):5280-4.

20. Toracchio S, Foyle A, Sroller V, Reed JA, Wu J, Kozinetz CA, et al. Lymphotropism of Merkel cell polyomavirus infection, Nova Scotia, Canada. Emerging infectious diseases. 2010;16(11):1702-9.

21. Teman CJ, Tripp SR, Perkins SL, Duncavage EJ. Merkel cell polyomavirus (MCPyV) in chronic lymphocytic leukemia/small lymphocytic lymphoma. Leuk Res. 2011;35(5):689-92.

22. Imajoh M, Hashida Y, Taniguchi A, Kamioka M, Daibata M. Novel human polyomaviruses, Merkel cell polyomavirus and human polyomavirus 9, in Japanese chronic lymphocytic leukemia cases. Journal of hematology \& oncology. 2012;5:25.

23. Schowalter RM, Pastrana DV, Pumphrey KA, Moyer AL, Buck CB. Merkel cell polyomavirus and two previously unknown polyomaviruses are chronically shed from human skin. Cell host \& microbe. 2010;7(6):509-15.

24. Fava P, Merlino C, Novelli M, Ponti R, Galliano I, Montanari P, et al. HPyV6, HPyV7 and TSPyV DNA sequences detection in skin disease patients and healthy subjects. Journal of the European Academy of Dermatology and Venereology. 2015:n/a-n/a.

25. Imajoh M, Hashida Y, Nakajima H, Sano S, Daibata M. Prevalence and viral DNA loads of three novel human polyomaviruses in skin cancers from Japanese patients. The Journal of dermatology. 2013;40(8):657-60.

26. Schrama D, Groesser L, Ugurel S, Hafner C, Pastrana DV, Buck CB, et al. Presence of human polyomavirus 6 in mutation-specific BRAF inhibitor-induced epithelial proliferations. JAMA dermatology. 2014;150(11):1180-6.

27. Scola N, Wieland U, Silling S, Altmeyer P, Stucker M, Kreuter A. Prevalence of human polyomaviruses in common and rare types of non-Merkel cell carcinoma skin cancer. The British journal of dermatology. 2012;167(6):1315-20.

28. Hampras SS, Locke FL, Chavez JC, Patel NS, Giuliano AR, Miller K, et al. Prevalence of cutaneous viral infections in incident cutaneous squamous cell carcinoma detected among chronic lymphocytic leukemia and hematopoietic stem cell transplant patients. Leuk Lymphoma. 2017:1-7.

29. Schrama D, Buck CB, Houben R, Becker JC. No evidence for association of HPyV6 or HPyV7 with different skin cancers. The Journal of investigative dermatology. 2012;132(1):239-41.

30. Duncavage EJ, Pfeifer JD. Human polyomaviruses 6 and 7 are not detectable in Merkel cell polyomavirus-negative Merkel cell carcinoma. Journal of cutaneous pathology. 2011;38(10):790-6.

31. Rennspiess D, Pujari S, Keijzers M, Abdul-Hamid MA, Hochstenbag M, Dingemans AM, et al. Detection of human polyomavirus 7 in human thymic epithelial tumors. J Thorac Oncol. 2015;10(2):360-6. 
32. Rozenblatt-Rosen O, Deo RC, Padi M, Adelmant G, Calderwood MA, Rolland T, et al. Interpreting cancer genomes using systematic host network perturbations by tumour virus proteins. Nature. 2012;487(7408):491-5.

33. Prado JCM, Monezi TA, Amorim AT, Lino V, Paladino A, Boccardo E. Human polyomaviruses and cancer: an overview. Clinics (Sao Paulo, Brazil). 2018;73(suppl 1):e558s.

34. Delbue S, Comar M, Ferrante P. Review on the relationship between human polyomaviruses-associated tumors and host immune system. Clin Dev Immunol. 2012;2012:542092.

35. White MK, Khalili K. Polyomaviruses and human cancer: molecular mechanisms underlying patterns of tumorigenesis. Virology. 2004;324(1):1-16.

36. Moens U, Rasheed K, Abdulsalam I, Sveinbjornsson B. The role of Merkel cell polyomavirus and other human polyomaviruses in emerging hallmarks of cancer. Viruses. 2015;7(4):1871-901.

37. Sroller V, Hamsikova E, Ludvikova V, Musil J, Nemeckova S, Salakova M. Seroprevalence rates of HPyV6, HPyV7, TSPyV, HPyV9, MWPyV and KIPyV polyomaviruses among the healthy blood donors. Journal of medical virology. 2016;88(7):1254-61.

38. Kamminga S, van der Meijden E, Feltkamp MCW, Zaaijer HL. Seroprevalence of fourteen human polyomaviruses determined in blood donors. PloS one. 2018;13(10):e0206273.

39. Nicol JT, Robinot R, Carpentier A, Carandina G, Mazzoni E, Tognon M, et al. Age-specific seroprevalences of merkel cell polyomavirus, human polyomaviruses 6, 7, and 9, and trichodysplasia spinulosa-associated polyomavirus. Clin Vaccine Immunol. 2013;20(3):363-8.

40. van der Meijden E, Bialasiewicz S, Rockett RJ, Tozer SJ, Sloots TP, Feltkamp MC. Different serologic behavior of MCPyV, TSPyV, HPyV6, HPyV7 and HPyV9 polyomaviruses found on the skin. PloS one. 2013;8(11):e81078.

41. Kamminga S, van der Meijden E, Wunderink HF, Touze A, Zaaijer HL, Feltkamp MCW. Development and Evaluation of a Broad Bead-Based Multiplex Immunoassay To Measure IgG Seroreactivity against Human Polyomaviruses. Journal of clinical microbiology. 2018;56(4).

42. Madeleine MM, Carter JJ, Johnson LG, Wipf GC, Davis C, Berg D, et al. Risk of squamous cell skin cancer after organ transplant associated with antibodies to cutaneous papillomaviruses, polyomaviruses, and TMC6/8 (EVER1/2) variants. Cancer medicine. 2014;3(5):1440-7.

43. Malhotra J, Waterboer T, Pawlita M, Michel A, Cai Q, Zheng W, et al. Serum biomarkers of polyomavirus infection and risk of lung cancer in never smokers. British Journal of Cancer. 2016;115(9):1131-9.

44. Vaillant V, Reiter A, Zimmermann M, Wagner HJ. Seroepidemiological analysis and literature review of the prevalence of Epstein-Barr virus and herpesvirus infections in pediatric cases with non-Hodgkin lymphoma in Central Europe. Pediatric Blood \& Cancer. 2019;66(7):e27752.

45. Orem J, Sandin S, Mbidde E, Mangen FW, Middeldorp J, Weiderpass E. Epstein-Barr virus viral load and serology in childhood non-Hodgkin's lymphoma and chronic inflammatory conditions in Uganda: implications for disease risk and characteristics. Journal of medical virology. 2014;86(10):1796-803.

46. Ji MF, Wang DK, Yu YL, Guo YQ, Liang JS, Cheng WM, et al. Sustained elevation of Epstein-Barr virus antibody levels preceding clinical onset of nasopharyngeal carcinoma. British journal of cancer. 2007;96(4):623-30. 
47. Shah KV, Viscidi RP, Alberg AJ, Helzlsouer KJ, Comstock GW. Antibodies to human papillomavirus 16 and subsequent in situ or invasive cancer of the cervix. Cancer epidemiology, biomarkers \& prevention : a publication of the American Association for Cancer Research, cosponsored by the American Society of Preventive Oncology. 1997;6(4):233-7.

48. Lehtinen M, Dillner J, Knekt P, Luostarinen T, Aromaa A, Kirnbauer R, et al. Serologically diagnosed infection with human papillomavirus type 16 and risk for subsequent development of cervical carcinoma: nested case-control study. BMJ (Clinical research ed). 1996;312(7030):537-9.

49. Karagas MR, Nelson HH, Sehr P, Waterboer T, Stukel TA, Andrew A, et al. Human papillomavirus infection and incidence of squamous cell and basal cell carcinomas of the skin. Journal of the National Cancer Institute. 2006;98(6):389-95.

50. Nguyen KD, Lee EE, Yue Y, Stork J, Pock L, North JP, VandergriffT, Cockerell C, Hosler GA, Pastrana DV, Buck CB, Wang RC. Human polyomavirus 6 and 7 are associated with pruritic and dyskeratotic dermatoses. J Am Acad Dermatol. 2017;76(5):932-940. doi: 10.1016/j.jaad.2016.11.035.

51. Canavan TN, Baddley JW, Pavlidakey P, Tallaj JA, Elewski BE. Human polyomavirus-7-associated eruption successfully treated with acitretin. Am J Transplant Off J Am Soc Transplant Am Soc Transplant Surg. 2018;18(5):1278-1284. doi: 10.1111/ajt.14634.

52. Ho J, Jedrych JJ, Feng H, Natalie AA, Grandinetti L, Mirvish E, Crespo MM, Yadav D, Fasanella KE, Proksell S, Kuan SF, Pastrana DV, Buck CB, Shuda Y, Moore PS, Chang Y. Human polyomavirus 7-associated pruritic rash and viremia in transplant recipients. J Infect Dis. 2015;211(10):1560-1565. doi: 10.1093/infdis/jiu524.

53. Purdie KJ, Proby CM, Rizvi H, Griffin H, Doorbar J, Sommerlad M, Feltkamp MC, Meijden EV, Inman GJ, South AP, Leigh IM, Harwood CA. The role of human papillomaviruses and polyomaviruses in BRAF-inhibitor induced cutaneous squamous cell carcinoma and benign Squamoproliferative lesions. Front Microbiol. 2018;9:1806. doi: 10.3389/fmicb.2018.01806.

54. Rascovan N, Monteil Bouchard S, Grob JJ, Collet-Villette AM, Gaudy-Marqueste C, Penicaud M, Lepidi H, Raoult D, Desnues C. Human Polyomavirus-6 infecting lymph nodes of a patient with an Angiolymphoid hyperplasia with eosinophilia or Kimura disease. Clin Infect Dis. 2016;62(11):14191421. doi: $10.1093 / \mathrm{cid} / \mathrm{ciw} 135$.

55. Hashida Y, Higuchi T, Nakajima K, Ujihara T, Murakami I, Fujieda M, Sano S, Daibata M. Human polyomavirus 6 with the Asian-Japanese genotype in cases of Kimura disease and Angiolymphoid hyperplasia with eosinophilia. J Invest Dermatol. 2020;140(8):1650-1653.e4. doi: 10.1016/j. jid.2019.12.027.

56. Sadeghi M, Wang Y, Ramqvist T, Aaltonen LM, Pyoria L, Toppinen M, et al. Multiplex detection in tonsillar tissue of all known human polyomaviruses. BMC Infect Dis. 2017;17(1):409. doi: 10.1186/ s12879-017-2479-5.

57. Herberhold S, Hellmich M, Panning M, Bartok E, Silling S, Akgul B, et al. Human polyomavirus and human papillomavirus prevalence and viral load in non-malignant tonsillar tissue and tonsillar carcinoma. Med Microbiol Immunol. 2017;206(2):93-103. doi: 10.1007/s00430-016-0486-6. 
58. Salakova M, Koslabova E, Vojtechova Z, Tachezy R, Sroller V. Detection of human polyomaviruses MCPyV, HPyV6, and HPyV7 in malignant and non-malignant tonsillar tissues. J Med Virol. 2016;88(4):695-702. doi: 10.1002/jmv.24385.

59. Rockett RJ, Sloots TP, Bowes S, O’Neill N, Ye S, Robson J, et al. Detection of novel polyomaviruses, TSPyV, HPyV6, HPyV7, HPyV9 and MWPyV in feces, urine, blood, respiratory swabs and cerebrospinal fluid. PLoS One. 2013;8(5):e62764. doi: 10.1371/journal.pone.0062764.

60. Zheng W-z, Wei T-1, Ma F-l, Yuan W-m, Zhang Q, Zhang Y-X, et al. Human polyomavirus type six in respiratory samples from hospitalized children with respiratory tract infections in Beijing, China. Virol J. 2015;12(1):166. 10.1186/s12985-015-0390-5

61. Delbue S, Elia F, Signorini L, Bella R, Villani S, Marchioni E, Ferrante P, Phan TG, Delwart E. Human polyomavirus $6 \mathrm{DNA}$ in the cerebrospinal fluid of an HIV-positive patient with leukoencephalopathy. J Clin Virol. 2015;68:24-27. doi: 10.1016/j.jcv.2015.04.016.

62. Delbue S, Franciotta D, Giannella S, Dolci M, Signorini L, Ticozzi R, et al. Human Polyomaviruses in the Cerebrospinal Fluid of Neurological Patients. Microorganisms. 2019;8(1):16. 10.3390/ microorganisms8010016.

63. Siebrasse EA, Bauer I, Holtz LR, Le BM, Lassa-Claxton S, Canter C, et al. Human polyomaviruses in children undergoing transplantation, United States, 2008-2010. Emerg Infect Dis. 2012;18(10):16761679. doi: 10.3201/eid1810.120359.

64. Chan JF, Tee KM, Choi GK, Zhu Z, Poon RW, Ng KT, et al. First detection and complete genome sequence of a phylogenetically distinct human polyomavirus 6 highly prevalent in human bile samples. J Inf Secur. 2017;74(1):50-9. 10.1016/j.jinf.2016.11.002.

65. Hashida Y, Higuchi T, Matsuzaki S, Nakajima K, Sano S, Daibata M. Prevalence and genetic variability of human polyomaviruses 6 and 7 in healthy skin among asymptomatic individuals. J Infect Dis. 2018;217(3):483-493. doi: 10.1093/infdis/jix516.

66. Higgins S, Nazemi A, Chow M, Wysong A. Review of nonmelanoma skin Cancer in African Americans, Hispanics, and Asians. Dermatol Surg. 2018;44(7):903-910. doi: 10.1097/DSS.0000000000001547.

67. Guy GP, Jr, Machlin SR, Ekwueme DU, Yabroff KR. Prevalence and costs of skin cancer treatment in the U.S., 2002-2006 and 2007-2011. Am J Prev Med. 2015;48(2):183-187. doi: 10.1016/j. amepre.2014.08.036.

68. Beckervordersandforth J, Pujari S, Rennspiess D, Speel EJ, Winnepenninckx V, Diaz C, et al. Frequent detection of human polyomavirus 6 in keratoacanthomas. Diagn Pathol. 2016;11(1):58. doi: 10.1186/ s13000-016-0509-z.

69. Bzhalava D, Johansson H, Ekstrom J, Faust H, Moller B, Eklund C, et al. Unbiased approach for virus detection in skin lesions. PLoS One. 2013;8(6):e65953. doi: 10.1371/journal.pone.0065953.

70. Kreuter A, Silling S, Dewan M, Stucker M, Wieland U. Evaluation of 4 recently discovered human polyomaviruses in primary cutaneous B-cell and T-cell lymphoma. Arch Dermatol. 2011;147(12):1449-1451. doi: 10.1001/archdermatol.2011.330.

71. Du-Thanh A, Foulongne V, Guillot B, Dereure O. Recently discovered human polyomaviruses in lesional and non-lesional skin of patients with primary cutaneous T-cell lymphomas. J Dermatol Sci. 2013;71(2):140-142. doi: 10.1016/j.jdermsci.2013.04.003. 
72. Toptan T, Yousem SA, Ho J, Matsushima Y, Stabile LP, Fernandez-Figueras MT, et al. Survey for human polyomaviruses in cancer. JCI Insight. 2016;1(2):e85562. 10.1172/jci.insight.85562.

73. Mulder FJ, Klufah F, Janssen FME, Farshadpour F, Willems SM, De Bree R, et al. Presence of human papillomavirus and Epstein-Barr virus, but absence of Merkel cell polyomavirus, in head and neck Cancer of non-smokers and non-drinkers. Front Oncol. 2021;10. 10.3389/fonc.2020.560434.

74. Martel-Jantin C, Filippone C, Cassar O, Peter M, Tomasic G, Vielh P, Brière J, Petrella T, AubriotLorton MH, Mortier L, Jouvion G, Sastre-Garau X, Robert C, Gessain A. Genetic variability and integration of Merkel cell polyomavirus in Merkel cell carcinoma. Virology. 2012;426(2):134-142. doi: 10.1016/j.virol.2012.01.018.

75. Antonsson A, Bialasiewicz S, Rockett RJ, Jacob K, Bennett IC, Sloots TP. Exploring the prevalence of ten polyomaviruses and two herpes viruses in breast cancer. PLoS One. 2012;7(8):e39842. doi: 10.1371/journal.pone.0039842.

76. Poluschkin L, Rautava J, Turunen A, Wang Y, Hedman K, Syrjänen K, Grenman R, Syrjänen S. Polyomaviruses detectable in head and neck carcinomas. Oncotarget. 2018;9(32):22642-22652. doi: 10.18632/oncotarget.25202.

77. Klufah F, Mobaraki G, Chteinberg E, Alharbi RA, Winnepenninckx V, Speel EJM, et al. High Prevalence of Human Polyomavirus 7 in Cholangiocarcinomas and Adjacent Peritumoral Hepatocytes: Preliminary Findings. Microorganisms. 2020;8(8):1125. 10.3390/microorganisms8081125.

78. Dolei A, Pietropaolo V, Gomes E, Di Taranto C, Ziccheddu M, Spanu MA, et al. Polyomavirus persistence in lymphocytes: prevalence in lymphocytes from blood donors and healthy personnel of a blood transfusion centre. The Journal of general virology. 2000;81(Pt 8):1967-73.

79. Krump NA, Liu W, You J. Mechanisms of persistence by small DNA tumor viruses. Current opinion in virology. 2018;32:71-9.

80. Zur Hausen H. Oncogenic DNA viruses. Oncogene. 2001;20(54):7820-3.

81. Moore PS, Chang Y. Why do viruses cause cancer? Highlights of the first century of human tumour virology. Nature reviews Cancer. 2010;10(12):878-89.

82. Shuda M, Arora R, Kwun HJ, Feng H, Sarid R, Fernandez-Figueras MT, et al. Human Merkel cell polyomavirus infection I. MCV T antigen expression in Merkel cell carcinoma, lymphoid tissues and lymphoid tumors. International journal of cancer. 2009;125(6):1243-9.

83. Houben R, Adam C, Baeurle A, Hesbacher S, Grimm J, Angermeyer S, et al. An intact retinoblastoma protein-binding site in Merkel cell polyomavirus large $T$ antigen is required for promoting growth of Merkel cell carcinoma cells. International journal of cancer. 2012;130(4):847-56.

84. Chimal-Ramírez GK, Espinoza-Sánchez NA, Fuentes-Pananá EM. Protumor activities of the immune response: insights in the mechanisms of immunological shift, oncotraining, and oncopromotion. Journal of oncology. 2013;2013:835956.

85. Mantovani A, Allavena P, Sica A, Balkwill F. Cancer-related inflammation. Nature. 2008;454(7203):43644.

86. Elinav E, Nowarski R, Thaiss CA, Hu B, Jin C, Flavell RA. Inflammation-induced cancer: crosstalk between tumours, immune cells and microorganisms. Nature Reviews Cancer. 2013;13(11):759-71. 
87. Balkwill FR, Mantovani A. Cancer-related inflammation: common themes and therapeutic opportunities. Semin Cancer Biol. 2012;22(1):33-40.

88. Coleman WB. Mechanisms of human hepatocarcinogenesis. Curr Mol Med. 2003;3(6):573-88.

89. Di Bisceglie AM. Hepatitis C and hepatocellular carcinoma. Hepatology. 1997;26(3 Suppl 1):34s-8s.

90. Brechot C, Kremsdorf D, Soussan P, Pineau P, Dejean A, Paterlini-Brechot P, et al. Hepatitis B virus (HBV)-related hepatocellular carcinoma (HCC): molecular mechanisms and novel paradigms. Pathologie-biologie. 2010;58(4):278-87.

91. Skinner GR. Transformation of primary hamster embryo fibroblasts by type 2 simplex virus: evidence for a "hit and run" mechanism. Br J Exp Pathol. 1976;57(4):361-76.

92. Prezioso C, Carletti R, Obregon F, Piacentini F, Manicone AM, Soda G, et al. Evaluation of Merkel Cell Polyomavirus DNA in Tissue Samples from Italian Patients with Diagnosis of MCC. Viruses. 2021;13(1).

93. Houben R, Grimm J, Willmes C, Weinkam R, Becker JC, Schrama D. Merkel cell carcinoma and Merkel cell polyomavirus: evidence for hit-and-run oncogenesis. The Journal of investigative dermatology. 2012;132(1):254-6.

94. Csoboz B, Rasheed K, Sveinbjørnsson B, Moens U. Merkel cell polyomavirus and non-Merkel cell carcinomas: guilty or circumstantial evidence? APMIS : acta pathologica, microbiologica, et immunologica Scandinavica. 2020;128(2):104-20.

95. Delbue S, Ferrante P, Provenzano M. Polyomavirus BK and prostate cancer: an unworthy scientific effort? Oncoscience. 2014;1(4):296-303.

96. Hori R, Murai Y, Tsuneyama K, Abdel-Aziz HO, Nomoto K, Takahashi H, et al. Detection of JC virus DNA sequences in colorectal cancers in Japan. Virchows Archiv : an international journal of pathology. 2005;447(4):723-30.

97. Wright CA, Nance JA, Johnson EM. Effects of Tat proteins and Tat mutants of different human immunodeficiency virus type 1 clades on glial JC virus early and late gene transcription. The Journal of general virology. 2013;94(Pt 3):514-23.

98. Behzad-Behbahani A, Klapper PE, Vallely PJ, Cleator GM, Khoo SH. Detection of BK virus and JC virus DNA in urine samples from immunocompromised (HIV-infected) and immunocompetent (HIV-non-infected) patients using polymerase chain reaction and microplate hybridisation. Journal of clinical virology : the official publication of the Pan American Society for Clinical Virology. 2004;29(4):224-9.

99. Knowles WA. Discovery and epidemiology of the human polyomaviruses BK virus (BKV) and JC virus (JCV). Advances in experimental medicine and biology. 2006;577:19-45.

100. Drop B, Strycharz-Dudziak M, Kliszczewska E, Polz-Dacewicz M. Coinfection with Epstein-Barr Virus (EBV), Human Papilloma Virus (HPV) and Polyoma BK Virus (BKPyV) in Laryngeal, Oropharyngeal and Oral Cavity Cancer. Int J Mol Sci. 2017;18(12).

101. Blacklow NR. Potentiation of an adenovirus-associated virus by herpes simplex virus type-2transformed cells. Journal of the National Cancer Institute. 1975;54(1):241-4.

102. Meier AF, Fraefel C, Seyffert M. The Interplay between Adeno-Associated Virus and its Helper Viruses. Viruses. 2020;12(6). 
103. Heilbronn R, Albrecht I, Stephan S, Bürkle A, zur Hausen H. Human cytomegalovirus induces JC virus DNA replication in human fibroblasts. Proceedings of the National Academy of Sciences of the United States of America. 1993;90(23):11406-10.

104. Fraase K, Hart J, Wu H, Pang X, Ma L, Grant F, et al. BK virus as a potential co-factor for HPV in the development of cervical neoplasia. Ann Clin Lab Sci. 2012;42(2):130-4.

105. Comar M, Bonifacio D, Zanconati F, Di Napoli M, Isidoro E, Martini F, et al. High prevalence of BK polyomavirus sequences in human papillomavirus-16-positive precancerous cervical lesions. Journal of medical virology. 2011;83(10):1770-6.

106. Borchert S, Czech-Sioli M, Neumann F, Schmidt C, Wimmer P, Dobner T, et al. High-affinity Rb binding, p53 inhibition, subcellular localization, and transformation by wild-type or tumor-derived shortened Merkel cell polyomavirus large T antigens. Journal of virology. 2014;88(6):3144-60.

107. Sullivan CS, Pipas JM. T antigens of simian virus 40: molecular chaperones for viral replication and tumorigenesis. Microbiol Mol Biol Rev. 2002;66(2):179-202.

108. DeCaprio JA, Ludlow JW, Figge J, Shew JY, Huang CM, Lee WH, et al. SV40 large tumor antigen forms a specific complex with the product of the retinoblastoma susceptibility gene. Cell. 1988;54(2):275-83.

109. Sheng Q, Denis D, Ratnofsky M, Roberts TM, DeCaprio JA, Schaffhausen B. The DnaJ domain of polyomavirus large $\mathrm{T}$ antigen is required to regulate $\mathrm{Rb}$ family tumor suppressor function. Journal of virology. 1997;71(12):9410-6.

110. Wu JH, Simonette RA, Nguyen HP, Rady PL, Tyring SK. Molecular mechanisms supporting a pathogenic role for human polyomavirus 6 small $\mathrm{T}$ antigen: Protein phosphatase $2 \mathrm{~A}$ targeting and MAPK cascade activation. Journal of medical virology. 2017;89(4):742-7.

111. Janssens V, Goris J, Van Hoof C. PP2A: the expected tumor suppressor. Curr Opin Genet Dev. 2005;15(1):34-41.

112. Howley PM, Pfister HJ. Beta genus papillomaviruses and skin cancer. Virology. 2015;479-480:290-6. 
CHAPTER 3

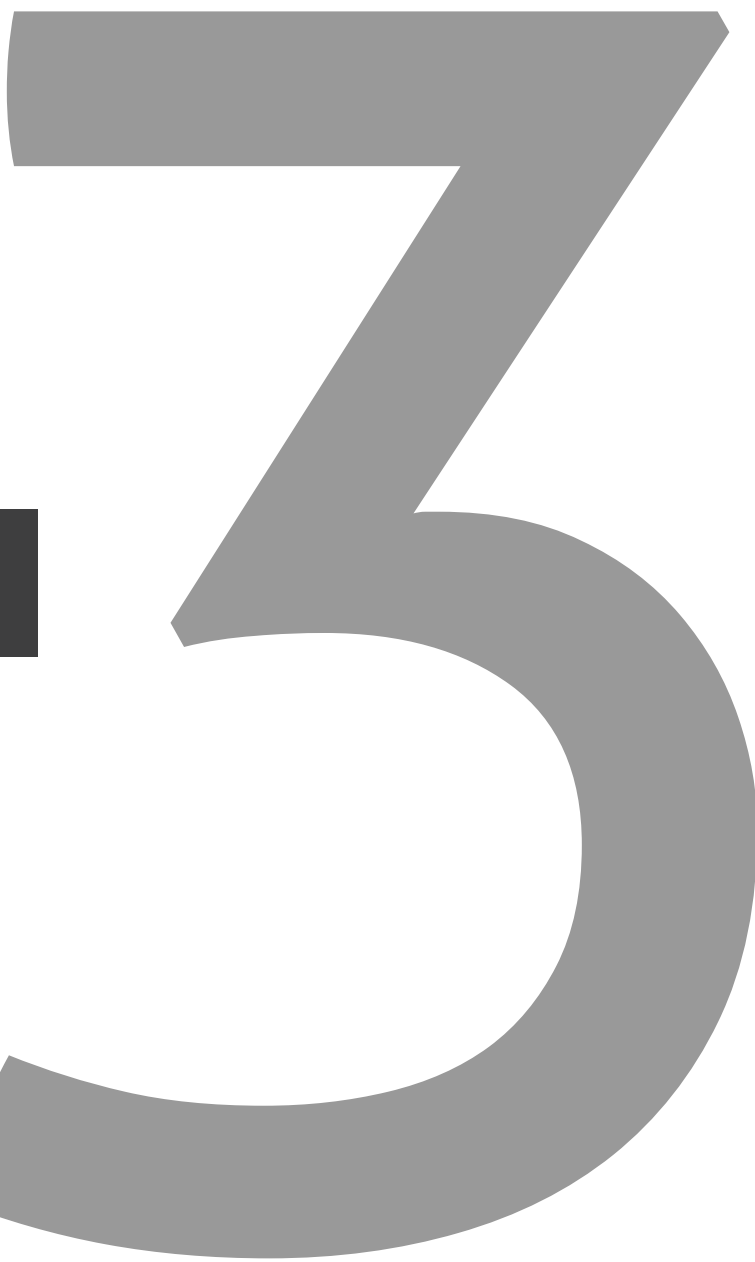




\section{High Prevalence of Human Polyomavirus 7 in Cholangiocarcinomas and Adjacent Peritumoral Hepatocytes: Preliminary Findings}

Faisal Klufah, Ghalib Mobaraki, Emil Chteinberg, Raed A. Alharbi, Véronique Winnepenninckx, Ernst Jan M. Speel, Dorit Rennspiess, Steven W. Olde Damink, Ulf P. Neumann, Anna Kordelia Kurz, Iryna Samarska, and Axel zur Hausen 


\section{Graphical abstract:}

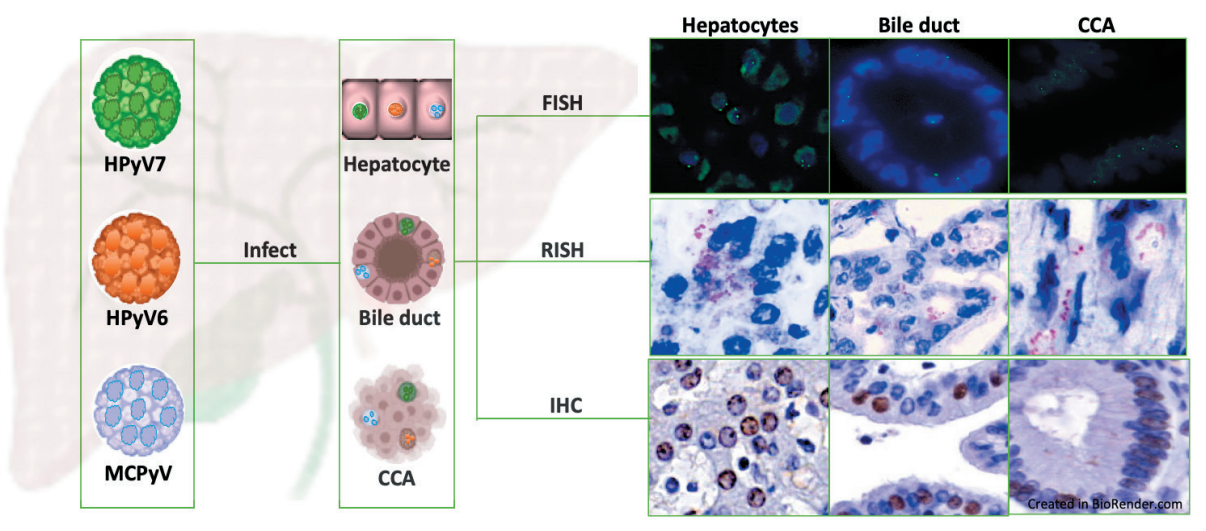

\section{ABSTRACT}

Cholangiocarcinoma (CCA) is a rare biliary-duct malignancy with poor prognosis. Recently, the presence of the human polyomavirus 6 (HPyV6) has been reported in the bile of diverse hepatobiliary diseases, particularly in the bile of CCA patients. Here, we investigated the presence of novel HPyVs in CCA tissues using diverse molecular techniques to assess a possible role of HPyVs in CCA. Formalin-Fixed Paraffin-Embedded (FFPE) tissues of 42 CCA patients were included in this study. PCR-based screening for $\mathrm{HPyVs}$ was conducted using degenerated and $\mathrm{HPyV}$-specific primers. Following that, we performed FISH, RNA in situ hybridization (RNA-ISH), and immunohistochemistry (IHC) to assess the presence of HPyVs in selected tissues. Of all 42 CCAs, 25 (59\%) were positive for one $\mathrm{HPyV}$, while 10 (24\%) CCAs were positive for $2 \mathrm{HPyV}$ simultaneously, and $7(17 \%)$ were negative for HPyVs. Of the total 35 positive CCAs, 19 (45\%) were positive for HPyV7, 4 (9\%) for HPyV6, 2 (5\%) for Merkel cell polyomavirus (MCPyV), 8 (19\%) for both HPyV7/MCPyV, and 2 (5\%) for both HPyV6/HPyV7 as confirmed by sequencing. The presence of viral nucleic acids was confirmed by specific FISH, while the RNA-ISH confirmed the presence of HPyV6 on the single-cell level. In addition, expression of $\mathrm{HPyV7}$, HPyV6, and MCPyV proteins were confirmed by IHC. Our results strongly indicate that $\mathrm{HPyV7}$, HPyV6, and MCPyV infect bile duct epithelium, hepatocytes, and CCA cells, which possibly suggest an indirect role of these viruses in the etiopathogenesis of CCA. Furthermore, the observed hepatotropism of these novel $\mathrm{HPyV}$, in particular $\mathrm{HPyV} 7$, might implicate a role of these viruses in other hepatobiliary diseases.

\section{KEYWORDS}

HPyV7; HPyV6; small DNA viruses; non-neoplastic hepatocytes; cancer; bile duct; Merkel cell polyomavirus; tumorigenesis 


\section{INTRODUCTION}

Cholangiocarcinoma (CCA) is a rare but very aggressive biliary duct neoplasia with a very poor prognosis ${ }^{1}$. The etiology of CCA is still largely obscure. However, several risk factors have been identified to be associated with the etiopathogenesis of CCA, e.g., primary sclerosing cholangitis, hepatolithiasis, inflammatory bowel disease, cirrhosis, diabetes, and biliary-duct cysts. Additionally, the role of environmental factors, e.g., toxins and smoking and microbiological infections, including hepatic parasites (flukes), and Hepatitis $\mathrm{B}$ and $\mathrm{C}$ viruses has been established in the etiopathogenesis of CCA ${ }^{1-3}$. Although CCA is rather uncommon in Western countries, the incidence of CCA has been steadily-yet inexplicably_rising during the last few decades ${ }^{1}$. Chan et al. recently reported the presence of the novel human polyomavirus 6 (HPyV6) in the bile of Chinese patients with diverse hepatobiliary diseases ${ }^{4}$.

Human polyomaviruses (HPyVs) are small DNA viruses in which e.g., BK polyomavirus $(\mathrm{BKPyV})$ and JC polyomavirus (JCPyV) have been identified since the early 1970s s,6. The current number of members of HPyVs has recently been expanded to fourteen, most of which have been identified within the past twelve years ${ }^{7,8}$. However, the only $\mathrm{HPyV}$ that has been identified as a human tumor virus is the Merkel cell polyomavirus $(\mathrm{MCPyV})^{9}$. Approximately $80 \%$ of Merkel cell carcinoma (MCC) cases are caused by clonal integration of MCPyV in the tumor genome of this highly malignant MCC. In addition, MCPyV-positive MCCs harbor tumor-specific oncogenic mutations in the viral oncogene encoding large tumor antigen $(\mathrm{LTAg})^{7,10-13}$.

Here, we assessed the prevalence of human polyomavirus 7 (HPyV7), HPyV6, and the oncogenic MCPyV in paraffin-embedded tissue sections (FFPE) of patients diagnosed with CCA, using diverse molecular techniques.

\section{MATERIALS AND METHODS}

\subsection{Patients and Specimens}

Forty-two formalin fixed and paraffin embedded (FFPE) tissue specimens of CCA patients collected at the Department of Pathology, Maastricht University Medical Centre+ (Maastricht Pathology Tissue Collection number: 2015-13) were included in this study. The study was approved by the Medical Ethics Review Committee of the Maastricht UMC+, the Netherlands (2019-0977), all tissue resections were collected and studied in accordance with the protocol of the Dutch Code of Conduct for Observational Research with Personal Data (2004) and Tissue ${ }^{14}$. 
Of these 42CCApatients, 25 (60\%) were male and 17 (40\%) female. The mean age was 65.2 years (range:29-85 yrs.). Three experienced pathologists (I.S., V.W., and A.z.H.) reviewed the histopathological and cytopathological features, and a consensus diagnosis of CCA subtypes was established. The recognition of the tubular non-neoplastic bile duct epithelium and neoplastic CCA cells has been performed by the above-mentioned pathologists.

DNA was isolated from all 42 FFPE tissues using techniques as previously described ${ }^{15}$. In brief, tissue sections were deparaffinized with xylene and the DNA was then extracted using the protocol of Genomic DNA from a tissue kit by Macherey-Nagel. The DNA concentration was assessed using a spectrophotometer (NanoDrop 2000, Thermo Scientific., Wilmington, DE, USA). Per sample, 250 ng was added in a PCR reaction. All isolated DNAs were assessed for quality and integrity using multiplex primers (SCS: specimen control size) as described previously ${ }^{15,16}$.

\subsection{Degenerated PCR}

In order to screen for the presence of HPyVs DNA, we modified the degenerated primers as recently published by Chan et al. ${ }^{4}$. The primer modification was performed according to the multiple alignment of the conservative region which is located within the conserved region in LTAg of HPyVs genome (Supplementary Figure S4a,b). To validate these degenerate primer sets, diverse PyVs-carrying plasmids were used, in addition to the MCPyV-positive MCC cell line (MKL-1) as positive controls in the PCR analysis. Bioperformance certified water was used as a non-template negative control.

The first nineteen isolated DNAs were screened for the presence of HPyVs DNA using degenerate primers, followed by sequencing of the obtained PCR products. DNA sequences were compared with the reference sequences of the National Center for Biotechnology Information (NCBI) Entrez Nucleotide database using the NCBI Blast program.

\subsection{Specific HPyVs DNA PCR}

In addition to the degenerated PCR technique, the specific PCRs targeting different gene regions of $\mathrm{HPyV} 7, \mathrm{HPyV} 6$, and $\mathrm{MCPyV}$ were performed using the protocols as previously described ${ }^{15}$. We used PCR to test all 42 CCA DNAs to amplify small tumor antigen (sTAg) and large tumor antigen (LTAg) of HPyV7, while sTAg genes of HPyV6 were amplified, as well as for three MCPyV genes, i.e., LT3, VP1, and M1/M2. In addition, multiple sequence alignment was performed for each primer result using the Clustal Omega algorithm (by The European Bioinformatics Institute) to compare with the positive control sequencing in the reference sequences of NCBI. All HPyV specific primers used in this study are summarized in Table S2. 


\subsection{Fluorescence In Situ Hybridization (FISH)}

All FISH procedures were carried out as described previously ${ }^{15,17,18,19}$. Additionally, to check whether or not the FISH protocol earlier described by Hopman et al. was optimal for our CCA FFPE tissues cohort, we hybridized selected tissues with the alpha satellite (centromeric) DNA probe (Vysis-CEP 12 by Abbott Co., Abbott Park, IL, USA) to ensure the quality of the pretreatment for both parenchymal and non-parenchymal liver cells. The majority of FFPE specimens that were tested positive by DNA PCR for one of the HPyVs were tested by DNA FISH for the presence of HPyV6, or 7 or MCPyV to confirm the presence of the DNA in single cell level with an alternative technique. In addition, we evaluated and validated the viral DNA signals to confirm if the signals were specific. We treated the positive controls and selected CCA tissues from our cohort with a FISH protocol in combination with either DNase I or RNAse prior to hybridization. By adding DNase then retesting, we ensured that the signal captured was DNA-specific; and by adding RNase, we ensured that the signal was not RNA-specific. Positive controls revealed specific signals that diminished slightly when the tissue was treated with RNAse prior to hybridization. However, all specific viral DNA signals vanished completely in the positive controls when treated with DNAse I (Supplementary Figure S3a) prior to hybridization. No cross-reactivity for the probes of HPyV 6 or 7 or MCPyV was observed. In addition, tissues previously tested negative for viral DNA served as negative controls for FISH, and no signals were seen. Furthermore, no specific nuclear signals were generated by omission of the probe during the hybridization. Therefore, HPyV6, HPyV7, and $\mathrm{MCPyV}$ biotin probes appeared to be sufficiently specific to detect the specific virus DNA (Supplementary Figure S3a).

The FISH slides were analyzed by at least three members (F.K., E.J.S., and A.z.H.) and the results of all cases were discussed together with all authors according to the criteria as previously described by Hafkamp et al. ${ }^{20}$. In addition, the specificity of the FISH probes was assessed on the liver tissues, by both DNase I and RNAse treatment, as described above (Supplementary Figure S3b).

\subsection{RNA In Situ Hybridization (RISH)}

The RNAscope assay using complementary RNA probes for the localization of specific expression of HPyVs mRNA was applied to selected cases: A specific probe for both HPyV6 and 7 LTAg genes was designed by Advanced Cell Diagnostics (ACD). A total of 20 pairs of 50 bp pooled probe designed for the HPyV7 target region between 3688 and 4876 bp (V-Polyomavirus-HPyV7, Accession No: HM011566.1). Another 20 pairs of RNA probes for the HPyV6 target region 3786-4898 bp (V-Polyomavirus-HPyV6, Accession No: HM011563.1). The sections were pretreated using the RNAscope ${ }^{\bullet} \mathrm{HD}$ Red 2.5 Kit (Advanced Cell Diagnostics, Cat No. 322350, Newark, CA, USA) according to the manufacturer's instructions. 
A probe for human peptidylprolyl isomerase B (Hs-PPIB) mRNA expression was used as a positive control, while a probe for bacterial dihydrodipicolinate reductase gene (DapB) expression was used as a negative control. First, Hs-PPIB and DapB probes were applied to FFPE HeLa cells to validate the pretreatment and hybridization conditions according to ACD protocols ${ }^{21}$. In addition, the two control probes were applied to each tested CCA case to confirm the expression of Hs-PPIB RNA and no expression of DapB. All RISH slides were evaluated and graded according to the ACD protocols and photos were taken using the VENTANA iScan-HT slide scanner ${ }^{21}$.

\subsection{Immunohistochemistry (IHC)}

The following antibodies and dilutions were used: the monoclonal antibodies 2T10 directed against HPyV7-sTAg and 1T1 directed against HPyV6-sTAg were provided by C.Buck, NCI, Bethesda, MD, USA, and used at a 1:100 dilution in combination with the EnVision-FLEX ${ }^{\mathrm{TM}}$ visualization Kit (K8008, DAKO, Carpinteria, CA, USA) according to standard protocols ${ }^{15}$. $2 \mathrm{~T} 10-\mathrm{Ab}$ is known to weakly cross-react with HPyV6, but not with MCPyV. 1T1-Ab is known to cross-react with HPyV7 and MCPyV while 2T10 $\mathrm{Ab}$ is known to weakly cross-react with $\mathrm{HPyV}^{22}$.

In addition, the CM2B4 monoclonal antibody was used to detect LT-antigen expression of $\mathrm{MCPyV}$, which is more sensitive and specific than $2 \mathrm{~T} 10$ and $1 \mathrm{~T} 1 \mathrm{Ab}$ as described by Moshiri et al. (clone: CM2B4, dilution 1:50; Santa Cruz Biotechnology Inc., Santa Cruz, CA, USA) ${ }^{23}$. The protocol used for HPyV6 and HPyV7 IHC was described previously, as was the protocol used for CM2B4 MCPyV IHC ${ }^{15,24}$.

HPyV7-positive thymoma tissues were used as previously published ${ }^{15}$. Keratoacanthoma tissue positive for HPyV6 was used as a control for 1T1 IHC ${ }^{18}$. MCC cell line MKL-1 positive for $\mathrm{MCPyV}$ was used as a positive control for the CM2B 4 antibody. All slides were evaluated by three pathologists (I.S., V.W., and A.z.H.) and photos were taken from the scans obtained by the VENTANA iScan-HT slide scanner. IHC was performed in selected PCR virus-positive cases and randomly for selected PCR virus-negative cases.

The immunohistochemistry stainings were scored either microscopically or using digital slides. The whole tissue sections were evaluated for the presence of positive nuclei and cytoplasm in hepatocytes, large bile duct, portal tracts, and neoplastic CCA cells. The percentage of positive cells was assessed. Inflammatory infiltrate was also evaluated if it was present. The intensity of HPyVs staining was evaluated and scored as strong $(+++)$ if the positive nuclei were seen at $100 \times$ magnification, moderate $(++)$ if the positive nuclei were seen at $200 \times$ magnification and weak $(+)$ if positive nuclei were seen at $400 \times$ magnification. 
In all subanatomic histological compartments (hepatocytes, large bile duct, and tumor cells), the quantitative analysis of HPyV7 IHC positive cells was evaluated using morphometry by (I.S., F.K.) on digital slides. The slides were scanned with the VENTANA iScan-HT slide scanner (Roche Diagnostics Inc., Tucson, AZ, USA) at 400x magnification. The morphometry was performed by calculating positive nuclei in the epithelial lining of the portal bile ducts, hepatocytes, and tumor cells in the hotspots in 5 photos at $400 \times$ magnification (HPF, high power field). The average of positive nuclei in the five images was calculated, and the results were present as a number of positive cells per HPF.

\subsection{Statistical Analysis}

The overall survival (OS) curve was estimated using the Kaplan-Meier method, and differences between HPyV-positive and -negative CCA cases were evaluated using a log rank test. OS was defined as the time between the date of tumor biopsy and date of death. Follow-up data for 39 (93\%) patients were available, of which two patients were excluded due to the lack of survival data, only iCCA and pCCA were selected. Statistical analysis was performed with R-studio, and a p-value of $<0.05$ was considered to be statistically significant. The cohort was used to build the survival model. For feature selection, we first removed correlated features (Pearson's $r>0.90$ ), using the publicly available Caret package ${ }^{25}$. The remaining features were used to build a Cox proportional hazards model, using the publicly available rms package ${ }^{26}$. The hazards ratio was calculated from the regression coefficients.

\section{RESULTS}

\subsection{Histopathology and Specimen Quality}

All isolated DNAs of the FFPE blocks revealed sufficient DNA quality with PCR products $>300 \mathrm{bp}$ based on the results of the specimen control size (SCS) ladder PCR (Supplementary Figure S1a). A consensus histopathological diagnosis by three pathologists revealed that thirty $(71.4 \%)$ patients had intrahepatic-CCA (iCCA), eleven $(26.2 \%)$ had perihilar-CCA ( $\mathrm{pCCA}$ ), and one (2.4\%) had distal-CCA (dCCA). No virus-like cytopathic effects or changes were observed in the cholangiocytes or hepatocytes during the review of HPyVs positive patient tissue specimens. Overall, 55\% of patients were diagnosed at stage I, 26\% at stage II, $14 \%$ at stage III, and 5\% at stage IV.

\subsection{Screening for Human Polyomaviruses Using Degenerate and HPyVs- Specific PCR}

We used the MCPyV-positive MKL-1 cell line and diverse polyomaviruses (PyVs) plasmids as positive controls for PCR with the modified degenerated primers. Accordingly, 
the modified degenerated primers were able to amplify PyVs sequences from MCPyV, HPyV6, 7, 9, NJPyV, WUV, CaPyV, and BPyV plasmids as well as the MKL-1 cell line (Supplementary Figure S1b), as confirmed by subsequent sequencing of PCR products. Screening of the first 19 CCA cases with this PCR technique revealed that fifteen $(79 \%)$ cases were positive for one of the novel $\mathrm{HPyV}$ s as confirmed by sequencing. HPyV7 DNA was detected in twelve CCA cases (63\%), followed by MCPyV DNA in three $(16 \%)$ cases. Of interest, no HPyV6 DNA was detected in the CCAs using degenerated PCR (Figure 1a).

Using HPyV7-specific PCR for all CCA cases of the cohort, 62\% (26/42) of CCA tissues were tested positive for the LTAg in comparison to 26\% (11/42) CCAs which were tested positive for the sTAg DNA-PCR (Figure 1b) (Supplementary Figure S2a,b). In contrast, only $14 \%$ (6/42) of cases were positive by HPyV6-specific PCR, which were negative in the degenerated primer PCR. MCPyV-specific PCR revealed seven cases (17\%) positive for the tumor antigen common region (M1/M2), two (5\%) positive for the viral protein gene 1 (VP1), and three (7\%) for the LTAg gene (LT3) (Supplementary Figure S2c,d). All PCR products were confirmed by sequence analysis, with few nucleotide differences between the cases (Table 1).

In summary, the results of all PCR approaches revealed that 59\% (25/42) of CCA tissues were positive for one of the HPyVs, while 10 (24\%) CCA tissues were positive for $2 \mathrm{HPyVs}$, and 7 (17\%) CCA tissues were negative for any HPyVs. Of the total 35 positive CCA tissues, 19 (45\%) were positive for HPyV7, 4 (9\%) for HPyV6, 2 (5\%) for Merkel cell polyomavirus (MCPyV), 8 (19\%) for both HPyV7/MCPyV, and 2 (5\%) for both HPyV6/HPyV7 (Figures 1c, d). Combined, the results of the HPyV7-specific and modified degenerate primer PCR showed that 29 (69\%) CCA tissues were positive for HPyV7 as confirmed by sequencing. Degenerated primer PCR showed 3 (16\%) CCA tissues positive for $\mathrm{MCPyV}$ after sequencing. Both degenerated and $\mathrm{MCPyV}$-specific PCR combined detected 10 (24\%) CCA tissues positive for MCPyV (Table 1). In this CCA cohort, we did not observe an association of a specific HPyV with a specific CCA subtype. Only one HPyV type was detected in the samples of each CCA subtype, except for iCCA in which 10 (24\%) CCAs were simultaneously harboring two different HPyVs. Eight cases were coinfected with $\mathrm{HPyV} 7$ and MCPyV, and two cases were coinfected with HPyV6 and HPyV7 (Figure 1d).

\subsection{Fluorescence In Situ Hybridization (FISH)}

Selected FFPE tissues chosen for assessing the FISH pretreatment with the alpha satellite (centromeric) DNA probe quality revealed intense FISH signals in all parenchymal and non-parenchymal liver cells. Thus, the protocol was optimal and was used for subsequent HPyV FISH analyses without modifications (Supplementary Figure S3a). 


\section{a}

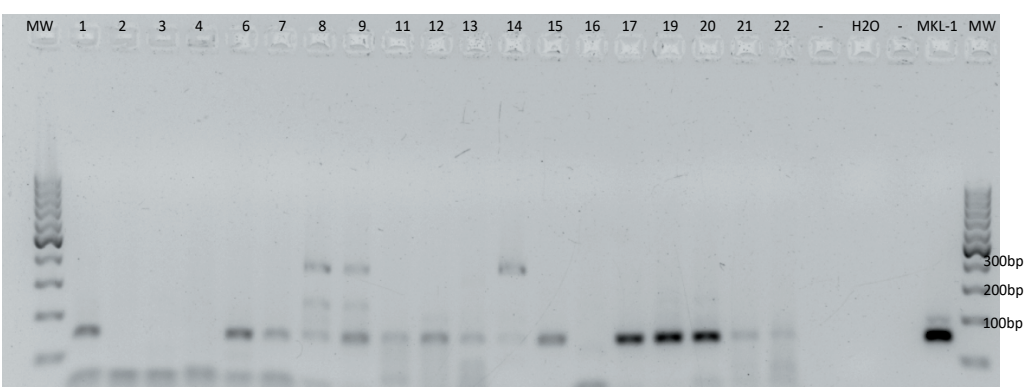

b

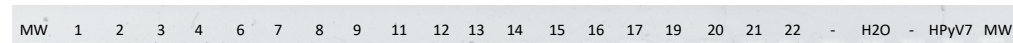

C

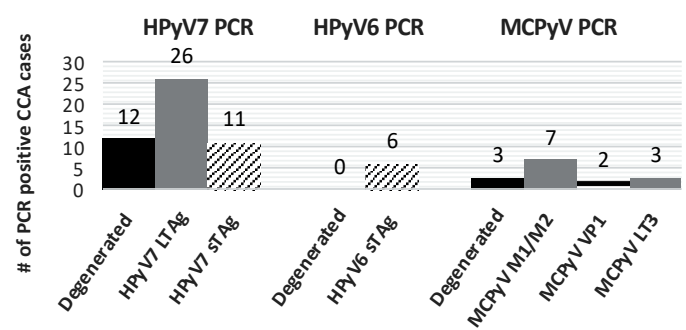

d

Figure 1. Degenerated and specific HPyV7 DNA-PCR. (a) The results of screening for HPyVs using FFPE tissues of Cholangiocarcinoma (CCA) by performing degenerated PCR-DNA. (b) Specific DNA-PCR amplifying HPyV7 sTAg (181 bp). (c) A chart summarizing the total HPyV results for both degenerated and specific DNA-PCR. The numbers on the gels refer to the number of CCA tissues of the respective CCA patients. (d) Venn diagram showing cases with two different HPyVs simultaneously. Abbreviations: Numbers on gels: represents the patient ID; $\mathrm{H}_{2} \mathrm{O}$ : water non-template negative control; MKL-1: MCPyV-positive Merkel cell carcinoma cell line used as a positive control; empty slot (no mastermix added to the gel); MW: molecular weight marker

Twelve CCA tissues were subjected to HPyV7-specific FISH analysis and four of them revealed specific nuclear signals in CCA tumor cells as well as non-neoplastic bile duct epithelium and hepatocytes (Table 2). HPyV7 DNA was detected as weak to moderate nuclear punctate signals in neoplastic CCA cells, and as moderate to strong punctate nuclear signals in non-neoplastic hepatocytes (Figure 2a). To assess if the FISH signals are DNA-specific, FISH analysis using the full length $\mathrm{HPyV} 7$ probe was performed on three tissue slides of case CCA1 (CCA patient with ID number 1). The first slide was hybridized with the $\mathrm{HPyV} 7$ probe resulting in $\mathrm{HPyV} 7$-specific green signals in 


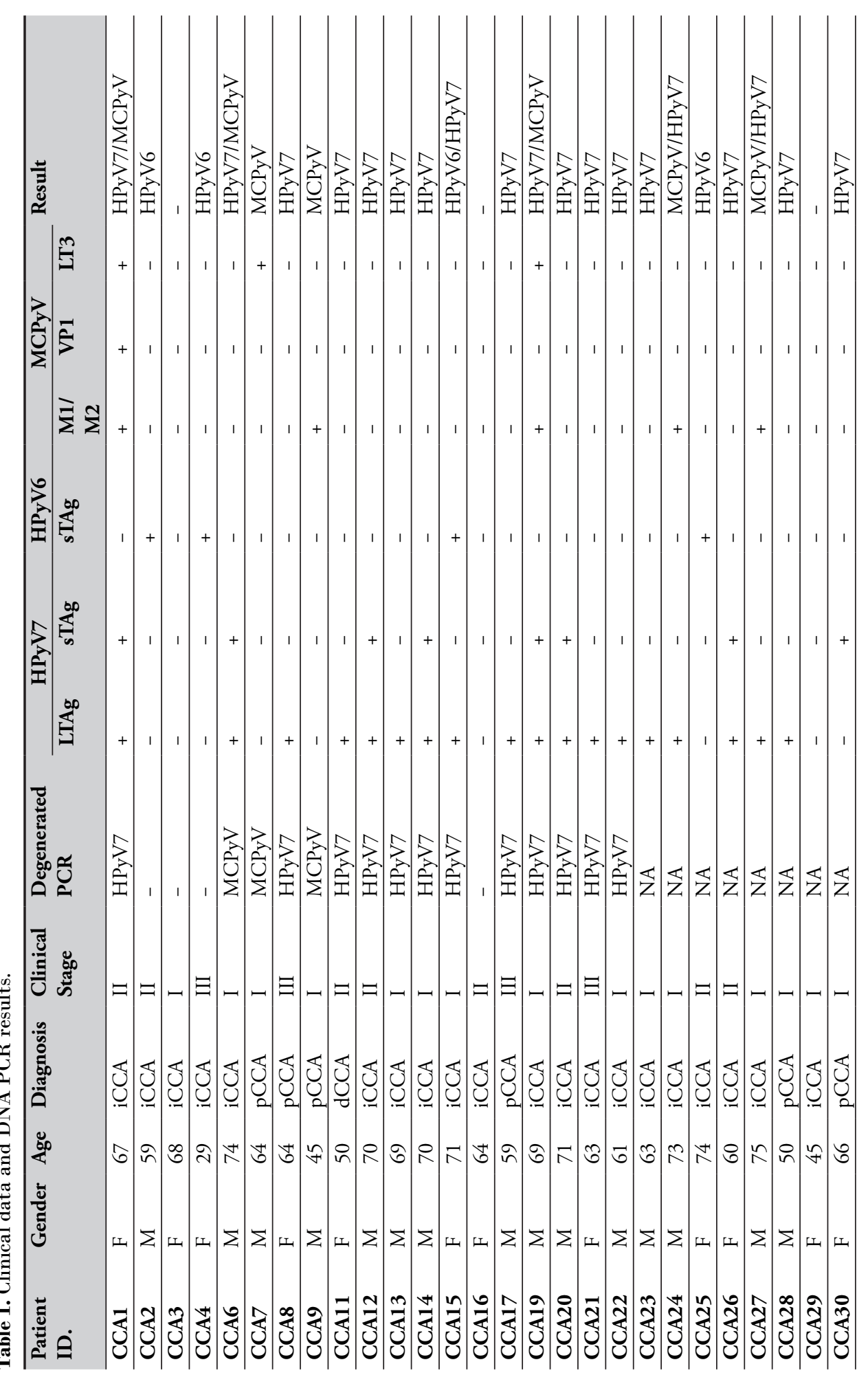




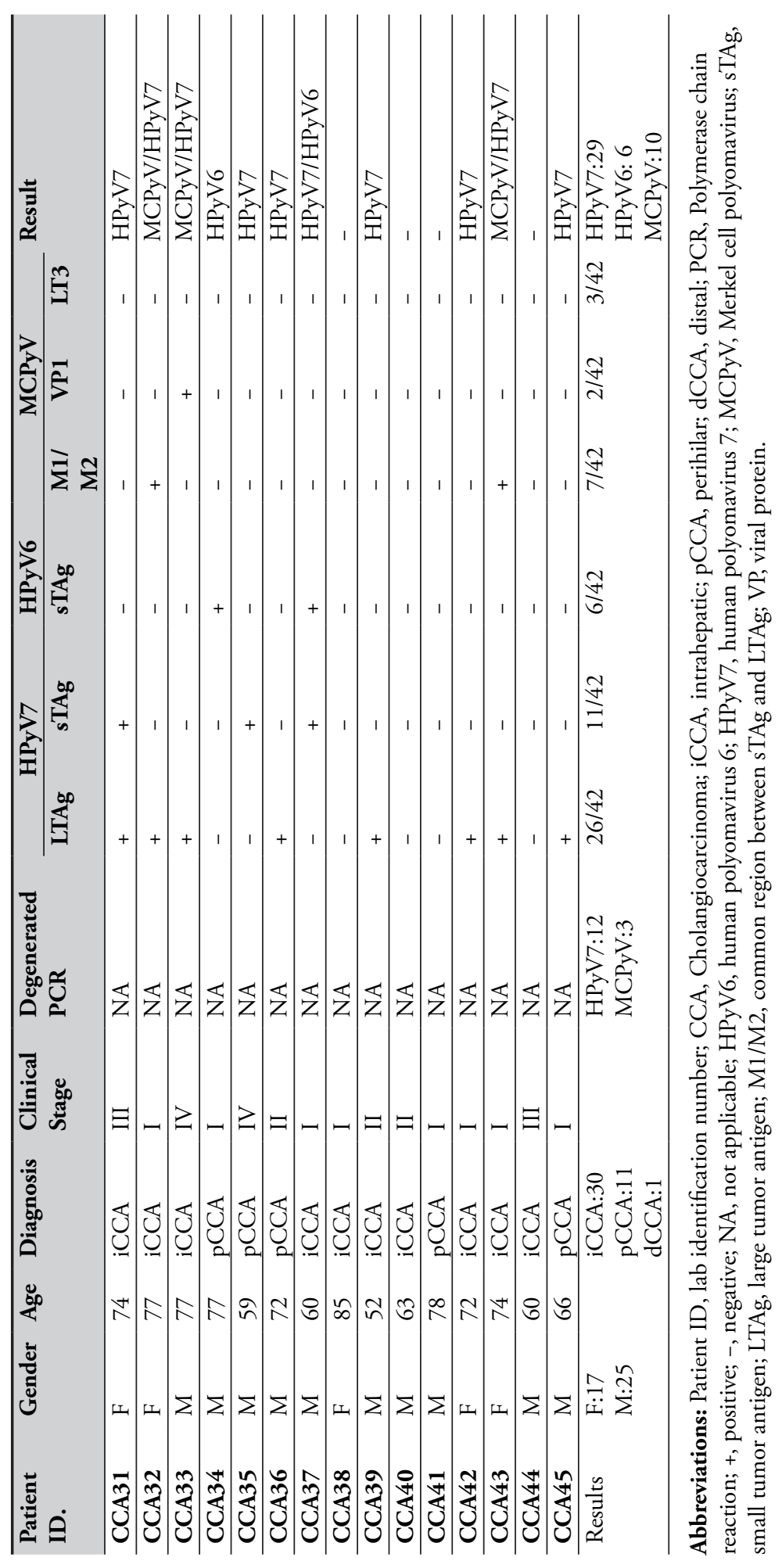



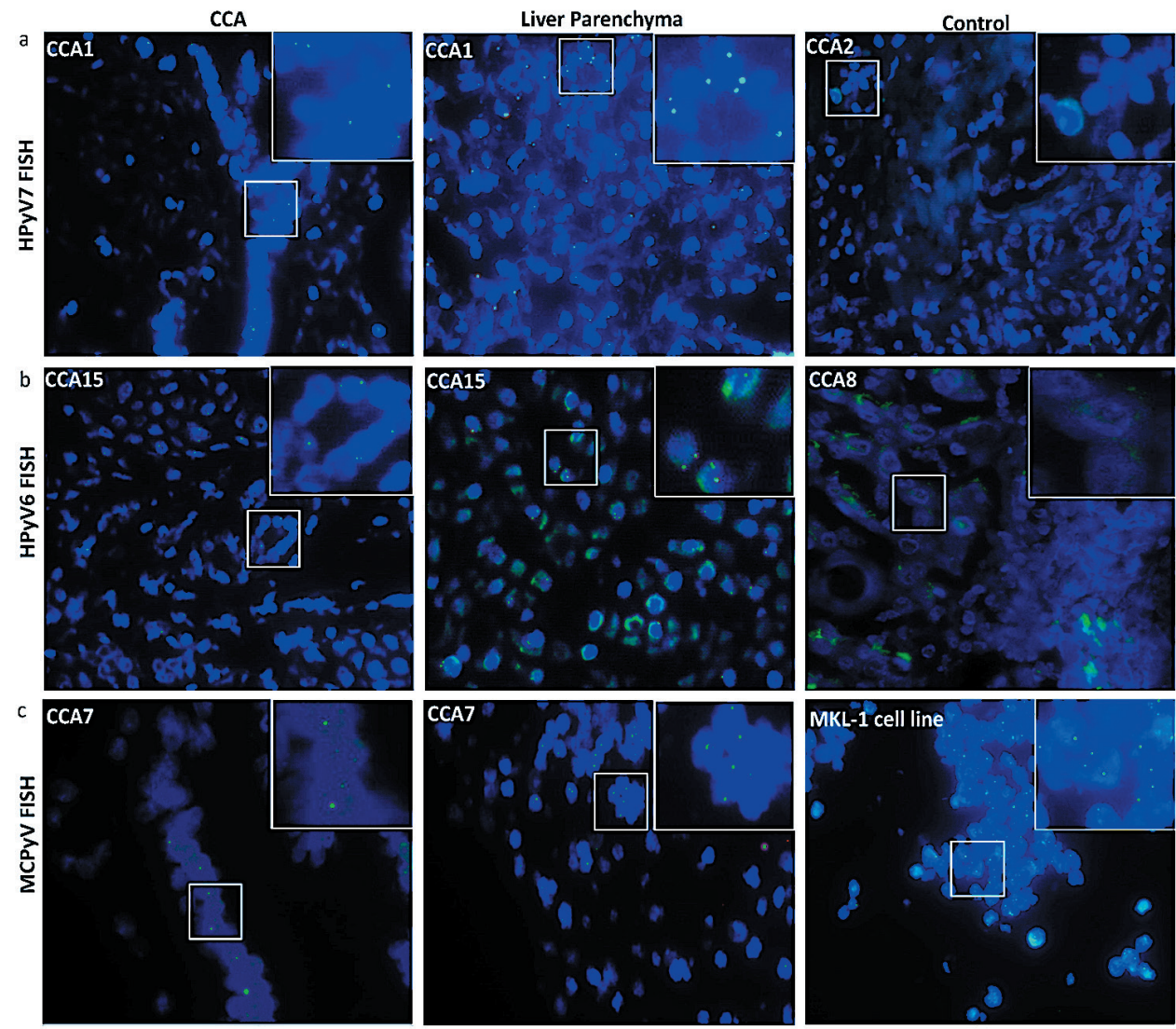

Figure 2. Detection of HPyV6, HPyV7, and MCPyV on the DNA in FFPE of CCA and non-neoplastic hepatocytes. Merged green (FITC) and blue (nuclei were counterstained with DAPI) show specific green signals. (a) Whole HPyV7 genome FISH probe performed on CCA1 tissue as a representative example of FISH for HPyV7 in CCA and non-neoplastic liver parenchyma cell while CCA2 tissue represents a negative case for HPyV7 DNA. (b) Whole HPyV6 genome FISH probe showed positive signals in both CCA and hepatocytes of CCA15, while the PCR-negative CCA8 case tissue revealed no signals. (c) Example of results of FISH specific nuclear of MCPyV in the nuclei of CCA epithelial, and the non-neoplastic hepatocytes. MKL-1 cell lines served as a positive control for the $\mathrm{MCPyV}$ probe. The images were taken at $630 \mathrm{x}$ magnification, a white square area was magnified $6 \mathrm{x}$ in the top right corner of each figure.

the bile duct tumor tissues as well as non-neoplastic hepatocytes. Pretreatment of the second slide with RNAse reduced the FISH signal intensity, while DNAse pretreatment diminished the specific signals almost completely as have been seen with the positive control (Supplementary Figure S3b).

All three HPyV6 DNA PCR positive cases showed specific nuclear HPyV6 DNA signals after FISH, although the dots were observed more often in the hepatocytes with normal morphology than in the CCA cells (Figure 2b). Additionally, the presence of MCPyV 


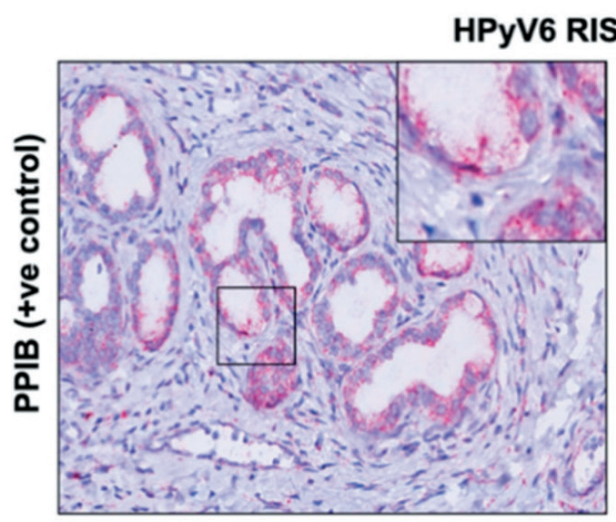

HPyV6 RISH for CCA2 case
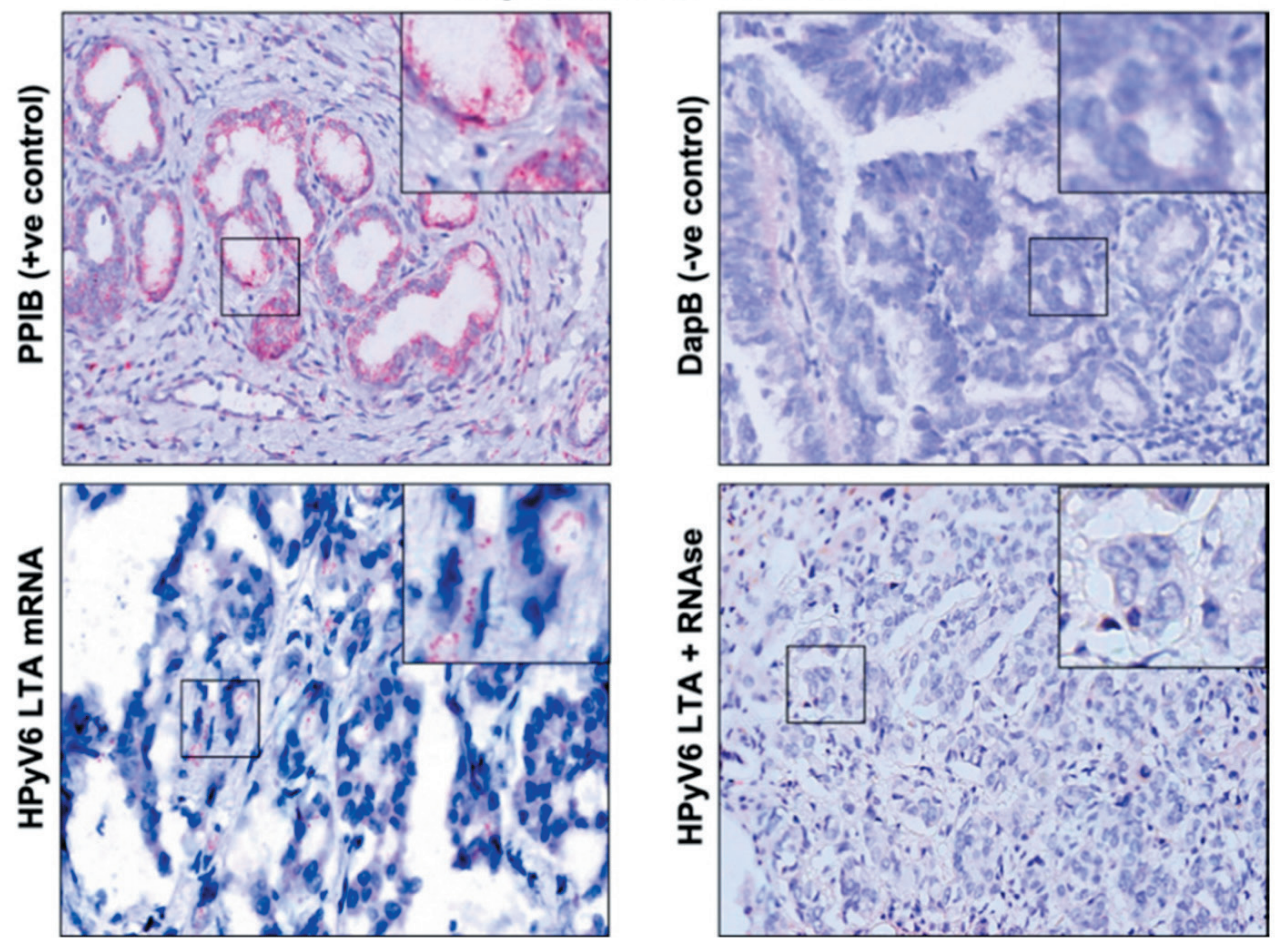

Figure 3. Detection of HPyV6 on the transcriptional level in FFPE CCA tissue by RNA-ISH, the CCA2 patient tissue section was hybridized with 20 sets labeled probes to detect HPyV6 LTAg mRNA using RNAscope RNA in situ hybridization assay. Additionally, FFPE hybridized with both the bacterial gene (DapB) as a negative control and the housekeeping gene (PPIB) as a positive control. Positive red signals were detected using fast red chromogen. HPyV6 LTA transcript seen as red signals in CCA and hepatocytes. No HPyV6 RNA was seen when the tissue was treated with RNAse, nuclei were counterstained with hematoxylin. The images were taken at 200x magnification, a black square area was magnified $6 \mathrm{x}$ in the top right corner of each figure.

was confirmed by FISH in four out of five MCPyV DNA positive PCR cases (Figure 2c) (Table 2).

\subsection{RNA In Situ Hybridization (RISH)}

The RNAscope technique was applied on 5 CCA tissues (2 HPyV6-positive CCA tissues and 3 HPyV6-negative CCA tissues, Table 2). The selection of these was based on the DNA PCR results and the availability of tissue specimens. HPyV6 mRNA was detected as specific red punctate spots in the nucleus and/or cytoplasm in two cases in both nonneoplastic hepatocytes and CCA cells, which had also tested positive by specific sTAgPCR, FISH, and immunohistochemistry (IHC) (Figure 3). Unfortunately, RNAscope for HPyV7 mRNA detection did not work in our hands. The background ratio in the selected tissues made an interpretation of specific HPyV7 mRNA signals impossible. 

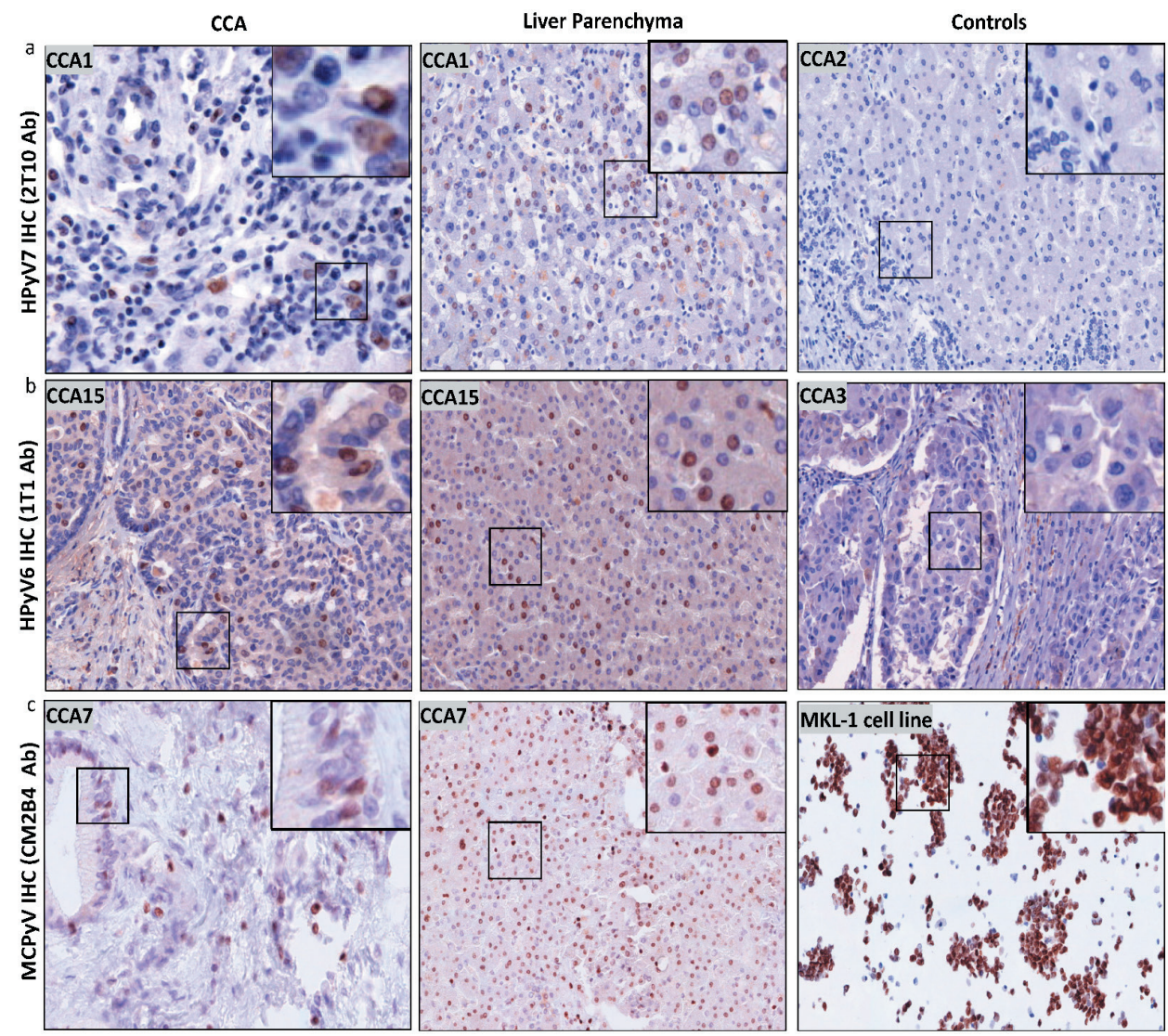

Figure 4. Detection of HPyV6, HPyV7, and MCPyV on the translational level in FFPE of CCA and non-neoplastic hepatocytes. (a) Representative examples of IHC using $2 \mathrm{t} 10$ antibodies showed the specific nuclear expression in the nucleus (brown) of both CCA and liver parenchyma. CCA2 tissue is an example of negative HPyV7. (b) In a representative IHC for CCA15 tissue, 1t1 antibodies show positivity in both CCA and hepatocytes, while no protein expression was seen in CCA3. (c) Example of results of IHC for $\mathrm{MCPyV}$, specific nuclear expression (brown) of MCPyV (CM2B4 antibody) in the nuclei of CCA epithelial, and the non-neoplastic hepatocytes. MKL1 cell line served as a positive for MCPyV antibodies. The images were taken at 200x magnification, a black square area was magnified $6 \mathrm{x}$ in the top right corner of each figure.

\subsection{Detection of HPyV7-sTAg, HPyV6-sTAg, and MCPyV-LTag Expression by Immunohistochemistry}

Twelve of sixteen CCA tissues were tested positive by HPyV7-IHC. Nine CCA tissues expressed HPyV7-sTAg in tumor cells, three of which showed strong nuclear expression in at least $20 \%$ of the tumor cells $(3 / 9 ; 33 \%)$ and six showed moderate to weak nuclear expression in five-ten percent of the tumor cells (Figure 4a). The expression of HPyV7sTAg within the histomorphological non-neoplastic adjacent large and small bile duct epithelium was also assessed and revealed nuclear positivity in eleven cases (11/16; 69\%) (Table 2). No strong expression was observed, and seven cases showed weak expression in 
Table 2. Fluorescence in situ hybridization, immunohistochemistry, and RNA in situ hybridization results.

\begin{tabular}{|c|c|c|c|c|c|c|c|c|c|c|}
\hline \multirow{3}{*}{$\begin{array}{l}\text { Patient } \\
\text { ID }\end{array}$} & \multicolumn{4}{|c|}{ HPyV7 } & \multicolumn{3}{|c|}{ HPyV6 } & \multicolumn{2}{|c|}{ MCPyV } & \multirow[t]{3}{*}{ Result } \\
\hline & \multirow[t]{2}{*}{ FISH } & \multicolumn{3}{|c|}{ IHC } & \multirow[t]{2}{*}{ FISH } & \multirow[t]{2}{*}{ RNA-Ish } & \multirow[t]{2}{*}{ IHC } & \multirow[t]{2}{*}{ FISH } & \multirow[t]{2}{*}{ IHC } & \\
\hline & & Hepatocyte & $\begin{array}{l}\text { Bile Duct } \\
\text { Epithelium }\end{array}$ & CCA & & & & & & \\
\hline CCA1 & ++ & +++ & ++ & ++ & - & NA & +++ & + & + & $\begin{array}{l}\text { HPyV7 } \\
\text { MCPyV }\end{array}$ \\
\hline CCA2 & - & - & - & - & + & + & ++ & - & NA & HPyV6 \\
\hline CCA4 & - & NA & NA & NA & + & NA & +++ & NA & NA & HPyV6 \\
\hline CCA6 & - & + & ++ & - & - & NA & ++ & + & ++ & $\begin{array}{l}\text { HPyV7 } \\
\text { MCPyV }\end{array}$ \\
\hline CCA7 & NA & - & - & - & - & - & +++ & ++ & +++ & $\mathrm{MCPyV}$ \\
\hline CCA8 & - & + & + & +++ & - & - & ++ & NA & NA & HPyV7 \\
\hline CCA9 & - & - & - & - & - & NA & +++ & - & ++ & MCPyV \\
\hline CCA11 & - & ++ & ++ & +++ & - & NA & NA & NA & - & HPyV7 \\
\hline CCA12 & - & + & ++ & + & - & NA & NA & NA & NA & HPyV7 \\
\hline CCA13 & NA & - & - & + & NA & NA & NA & NA & NA & HPyV7 \\
\hline CCA14 & + & +++ & + & - & NA & - & +++ & NA & NA & HPyV7 \\
\hline CCA15 & + & +++ & + & +++ & ++ & + & +++ & NA & NA & $\begin{array}{l}\text { HPyV6 } \\
\text { HPyV7 }\end{array}$ \\
\hline CCA16 & NA & - & - & - & NA & NA & - & - & NA & - \\
\hline CCA17 & NA & + & + & - & NA & NA & + & NA & NA & HPyV7 \\
\hline CCA19 & NA & + & + & + & NA & NA & - & + & + & $\begin{array}{l}\text { HPyV7 } \\
\text { MCPyV }\end{array}$ \\
\hline CCA20 & NA & + & + & + & NA & NA & + & NA & NA & HPyV7 \\
\hline CCA21 & + & + & + & + & - & NA & + & NA & NA & HPyV7 \\
\hline Total & $4 / 11$ & $11 / 16$ & $11 / 16$ & $9 / 16$ & $3 / 11$ & $2 / 5$ & $12 / 14$ & $4 / 7$ & $5 / 6$ & \\
\hline
\end{tabular}

Abbreviations: Patient ID, lab identification number; CCA, Cholangiocarcinoma; +, weak positive; ++, moderate positive; +++, strong positive; -, negative; NA, not applicable; HPyV6, human polyomavirus 6; HPyV7, human polyomavirus 7; MCPyV, Merkel cell polyomavirus; sTAg, small tumor antigen; LTAg, large tumor antigen; IHC, immunohistochemistry; FISH, fluorescence in situ hybridization; RNA-ish, RNA in situ hybridization.

less than $5 \%$ of bile ducts. Of interest, adjacent histomorphological non-neoplastic liver parenchyma also revealed specific nuclear positivity for HPyV7 in 11 cases (11/6; 65\%) (Figure 4a). Strong hepatocellular expression was seen in three cases, moderate intensity in one case, and weak staining in seven cases (Table 2). The percentage of positive nuclei varied from less than $5 \%$ up to approximately $80 \%$. No cytoplasmic expression was observed in neoplastic or non-neoplastic bile duct epithelium and hepatocytes, and no differences in zonal distribution were observed. In cases with a prominent inflammatory infiltrate, scattered lymphocytes also showed nuclear positivity for HPyV7. Furthermore, endothelium of the blood vessels was completely negative for HPyV7-sTAg expression.

Moreover, the number of HPyV7-positive nuclei in hepatocytes, bile duct epithelium, and tumor cells was counted in the hotspots in 5 high power fields (HPF; 400X) using morphometric analysis (Table S1). Thus, these findings confirm at least a low HPyV7 protein expression in at least a few positive cells per HPF in all positive IHC cases of CCA regardless of the CCA subtype and/or clinical stage. Moreover, periportal hepatocytes 
showed at least a focal positivity in all of these cases. In cases with reactive ductular reaction within the limiting plate of the portal tract, the newly formed small ducts also showed at least focal immunoreactivity.

Twelve of the selected fourteen CCA tissues were tested positive for HPyV6-sTAg IHC expression. However, more CCA tissues revealed HPyV6-IHC positivity than PCR due to the fact that $1 \mathrm{t} 1$ antibodies are well known to have cross-reactivity with $\mathrm{HPyV} 7$ and MCPyV. The intensity of the HPyV6-IHC staining was weak to moderate with a maximal $10 \%$ of positive cells (Figure 4b). No sTAg expression within the cytoplasm was observed, and no expression was found in endothelial cells of blood/lymphatic vessels. Of interest, twelve CCA tissues revealed specific nuclear HPyV6-sTAg expression in non-neoplastic hepatocytes $(12 / 14 ; 86 \%)$. Six cases revealed strong nuclear reactivity with percentages of positive nuclei between $20 \%$ and $80 \%$ (Figure $4 \mathrm{~b}$ ). No differences in zonal distribution were observed. Normal epithelial lining of bile duct epithelium was only weakly positive in 5 to $40 \%$ of the nuclei of cholangiocytes.

Six CCA tissues were tested for MCPyV-LTag expression by IHC, five of which showed nuclear staining $(5 / 6 ; 83.3 \%)$ (Figure 4c). Three CCA tissues revealed both weak nuclear positivity in maximal $30 \%$ of tumor cells. All of the hepatocytes showed a weak to moderate staining pattern (Figure 4c). One CCA tissue revealed cytoplasmic staining, which was interpreted as unspecific and thus negative. No differences in zonal distribution were seen. Normal epithelial lining of bile duct epithelium was only positive in three cases up to $30 \%$ of nuclei of bile duct epithelium (ranging between weak and strong intensity). No cytoplasmic staining was observed (Table 2).

\subsection{Overall Survival Analysis}

Statistics data analysis revealed no significant association between overall survival (OS) and HPyV7 ( $\mathrm{p}=0.726), \operatorname{HPyV6}(\mathrm{p}=0.366)$, and MCPyV ( $\mathrm{p}=0.953)$ in iCCA and pCCA. The hazards ratio and the $\mathrm{p}$-value are reported in Cox regression data (Table 3). The Kaplan-Meier curve showed no significant risk stratification for CCA patients (Figure 5).

Table 3. Cox regression model for multivariable analysis of iCCA and pCCA cohort.

\begin{tabular}{lll}
\hline Variable & Hazards Ratio & p-value \\
\hline Sex & 0.617888 & 0.521 \\
\hline Age & 1.004683 & 0.893 \\
\hline Subtype & 2.639623 & 0.174 \\
\hline Stage & 0.761135 & 0.551 \\
\hline HPyV7 & 0.741641 & 0.726 \\
\hline HPyV6 & 2.293245 & 0.366 \\
\hline MCPyV & 1.057001 & 0.953 \\
\hline
\end{tabular}



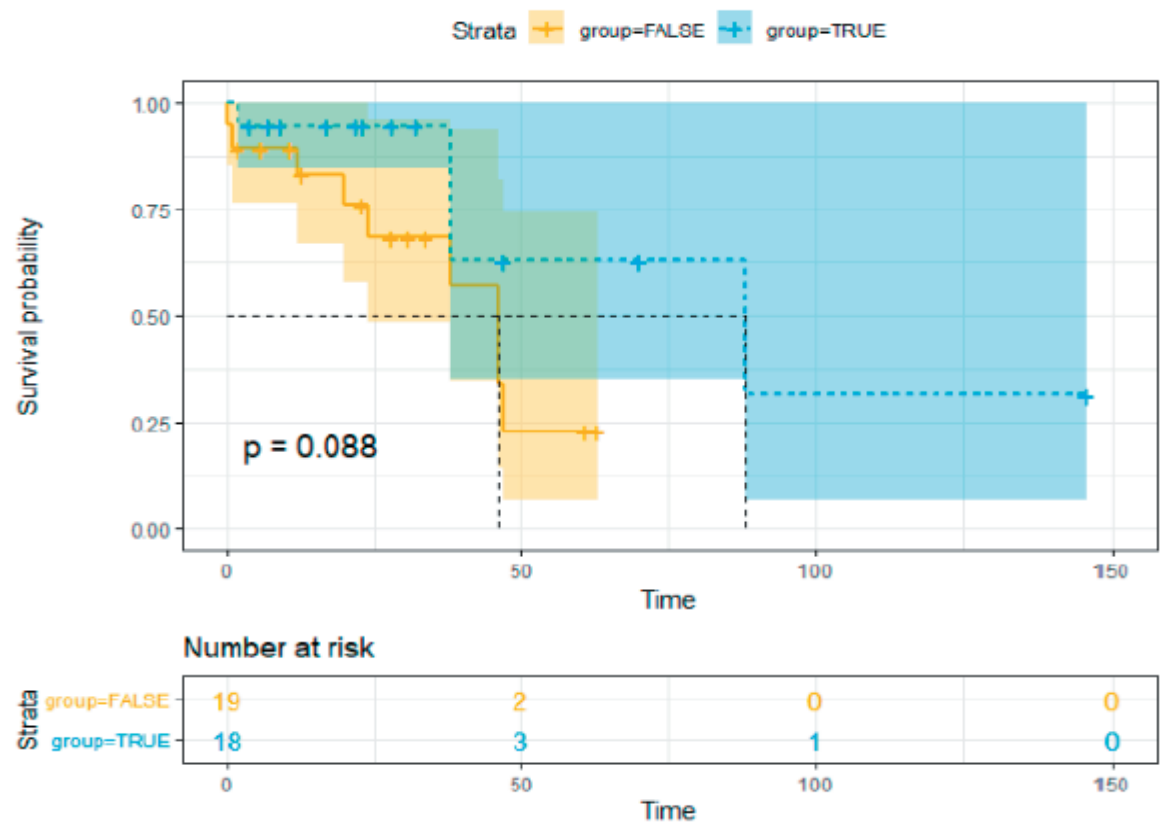

Figure 5. Kaplan-Meier method survival curve for overall survival.

\section{DISCUSSION}

The etiology of CCA is largely unknown. Here, we investigated the presence of HPyV7, 6 , and $\mathrm{MCPyV}$ in CCA tissue specimens, including adjacent histomorphological non-neoplastic bile duct epithelium and non-neoplastic hepatocytes, using diverse molecular detection techniques. We used a combined approach of a degenerated primer set for HPyVs and diverse HPyVs-specific PCRs, all PCRs were followed by sequence analysis. Degenerated PCR revealed to be a reliable detection of HPyVs in FFPE tissues demonstrating that the use of degenerated $\mathrm{HPyV}$ primers is applicable for screening purposes of FFPE tissues. (Figure 1a). To the best of our knowledge, this is the first study to perform FISH and IHC to confirm the presence of HPyV6, HPyV7, and MCPyV in the bile duct epithelium and hepatocytes, and also to show HPyV6 transcript by RNAISH in human tissues.

Compared to the prevalence of HPyV6 DNA in CCA tissues of our cohort 6 (14\%), HPyV7 DNA was detected much more frequently in 29 (69\%) of the CCA tissues. Of interest, some of the cases that tested HPyV7 DNA positive by degenerate LTAg PCR were negative by specific HPyV7 DNA-PCR targeting sTAg. However, assessing the whole cohort with a specific LTAg HPyV7 DNA-PCR within the conserved region which is targeted by the degenerated primers revealed a good agreement (Figure S2). This apparent incongruency of HPyVs DNA (LTAg vs sTAg) detection by PCR in FFPE 
tissue using different primer pairs is a well-known phenomenon. It is most likely due to the abundance of the diverse targeted sequences of the viral genome and is in line with prior observations of us and others in the detection of HPyVs such as MCPyV, HPyV6, and $\mathrm{HPyV} 7^{4,11,27,28}$. We have proven this discrepant phenomenon by using two specific HPyV7-DNA PCR targeting different regions (sTAg and LTAg). Our results showed that the rate of detection of specific HPyV7 LTAg is higher than the detection of sTAg, matching the results of the degenerated PCR.

An explanation for the discrepancy between high HPyV7 prevalence found by us and high HPyV6 prevalence found by Chan et al. might be the primary source of the DNA, i.e., primary CCA tissues versus bile fluids of CCA patients, respectively, or due to variation in the ethnical and geographical distribution of both cohorts. Recent studies have assessed HPyVs serum antibody levels across a range of age groups including one from the Netherlands. HPyV7 and HPyV6 were found to be highly seroprevalent in all age groups $29,30,31,32,33$. HPyV6 seroprevalence was slightly higher than HPyV7 and MCPyV. However, the mean age in our patient cohort was 65.2 years compared to 77 years in the study of Chan et al., largely ruling out age related effects to explain these diverse findings.

In addition to $\mathrm{HPyV} 7$ specific DNA PCR, we were able to visualize the presence of HPyV7 by FISH and IHC. The FISH results were in good agreement with the PCR results and allowed the identification of the virus at the single cell level within the histomorphological context. FISH revealed the presence of HPyV7 within non-neoplastic hepatocytes and bile duct epithelium as well as CCA tumor tissue (Figure 2a). However, not all tumor cells revealed the presence of HPyV7 DNA by FISH. On the protein level using the $2 \mathrm{t} 10 \mathrm{IHC}$ antibody, nine CCA cases revealed an accumulation of the HPyV7 sTAg in tumor cells. Compared to non-neoplastic bile duct epithelium as well as adjacent non-neoplastic hepatocytes, eleven cases showed expression of HPyV7 sTAg. Finding less expression in CCA tumor cases might be explained by the fact that CCA can be derived from different cellular origins, including mature hepatocytes ${ }^{34,35}$. Due to the different origins of cholangiocytes, we have seen some CCAs which were positive and some which were negative. Therefore, one can speculate that $\mathrm{HPyV7}$ might infect hepatocytes at an early stage, considering that hepatocytes have the potential to differentiate between bile ducts or CCA. CCA cells with negative HPyV7 expression might differentiate from the non-hepatocyte origin.

Unfortunately, we were unable to detect specific mRNA signals for HPyV7 RISH due to technical reasons, which could be explained by two possibilities: the background ratio in the selected tissues made an interpretation of specific HPyV7 mRNA signals impossible; or the selected target sequences of the HPyV7 probe need to be adapted. 
The above findings indicate that $\mathrm{HPyV} 7$ is most probably not directly related to the etiopathogenesis of CCA. However, the presence of HPyV7 in only a part of the tumor cells may also have been caused by technical restrictions of the FISH and IHC assays. The detection sensitivity of these assays is dependent on optimal pre-analytic tissue processing and preservation of nucleic acid quality and protein epitopes, which sometimes can be limited in FFPE tissues.

In the underlying study, a relatively low $14 \%$ prevalence of $\mathrm{HPyV} 6$ was detected in CCA tissues by HPyV6 specific PCR, which is lower than the $27 \%$ reported by Chan et al. in the bile fluid of CCA patients. By using the RNAscope technique, we were able to demonstrate HPyV6 transcripts on the single cell level in both non-neoplastic hepatocytes and CCA cells. This is in line with the DNA FISH and sTAg IHC results. The IHC results, however, need to be interpreted with care, since we also detected the HPyV6 sTAg expression in HPyV6 PCR-negative but HPyV7- and MCPyV- PCR positive cases, which is most likely due to the known cross-reactivity of this antibody with HPyV7 and $\mathrm{MCPyV}^{22}$.

$\mathrm{MCPyV}$ was found in $24 \%$ of CCAs by both degenerated and specific DNA PCR, which is consistent with previous reports ${ }^{36,37}$. Loyo and colleagues reported the presence of MCPyV DNA by qPCR in five (33\%) out of fifteen normal liver tissues, ten (63\%) out of sixteen liver cancers, and two (100\%) out of two biliary cirrhosis cases ${ }^{36}$. Additionally, the prevalence of $\mathrm{MCPyV}$ has been reported in three (24\%) of 124 nonmalignant liver tissues by generic polyomavirus PCR ${ }^{37}$. MCPyV FISH analysis was able to detect the virus DNA in CCA tumors as well as non-neoplastic hepatocytes and bile duct epithelium (Figure 2c), showing relatively weak signals similar to the general weak to faint amplicons detected by PCR. On the protein level, weak immunostaining of MCPyV LTAg viral proteins within both the CCA neoplastic cell and the normal looking bile duct epithelium was also observed. Thus, all three detection methods provided evidence for the presence of MCPyV in a subset of CCAs and normal liver and bile duct tissue. It is well known that malignant tumors associated with DNA viruses have a better survival as reported previously ${ }^{38,39}$. Our study revealed that iCCA and pCCA carrying HPyV7, HPyV6, or $\mathrm{MCPyV}$ DNA did not predict an association with overall survival and further larger studies are needed to confirm our findings.

The contribution of HPyV 7 to CCA carcinogenesis cannot be definitely elucidated by the present data. However, it remains speculation in as much as $\mathrm{HPyV} 7$ possibly contributes to CCA carcinogenesis. This might be based on a direct HPyV7-interaction and subsequent deactivation of $\mathrm{p} 53$, as recently reported by Rozenblatt-Rosen et al. ${ }^{40}$, or alternatively, by clonal integration of the $\mathrm{HPyV} 7$ genome with or without tumor-specific viral mutations as has been shown for MCPyV-positive MCCs ${ }^{11,41}$. The latter seems to 
be rather unlikely for HPyV7-positive CCAs as one would then expect a high expression of viral proteins in every tumor cell. The most likely contribution of HPyV7 and maybe other HPyVs is an indirect mechanism via the induction of (chronic) inflammation as has been convincingly shown for Hepatitis B and $\mathrm{C}$ viruses ${ }^{1}$.

There are some limitations to our study. These limitations are mainly due to the rarity of CCA which also explains the sample size of our patient cohort $(n=42)$. Of course, it would be of clinical interest to further elucidate possible other correlations between these HPyVs and clinical endpoints of CCA.

\section{CONCLUSIONS}

Our results strongly indicate that $\mathrm{HPyV} 7, \mathrm{HPyV} 6$, and $\mathrm{MCPyV}$ infect non-neoplastic hepatocytes, bile duct epithelium, and CCA lesions. Although all three HPyVs are hepatotropic, $\mathrm{HPyV} 7$ was more frequently detected in both neoplastic and non-neoplastic cells in our CCA subset compared to HPyV6 and MCPyV. To the best of our knowledge, this is the first study to report the presence of HPyV7, HpyV6, and MCPyV on the single cell level in liver tissues of non-neoplastic bile duct epithelium and hepatocytes and CCA. We used diverse molecular techniques to assess the presence of these viruses. Although our results might point to indirect role of these HPyVs to CCA carcinogenesis, possibly by inflammation, the role of these HPyVs in the etiopathogenesis of CCA needs to be further elucidated. Furthermore, the frequent finding of HPyVs, especially HPyV7 in liver tissues might also point to a possible role of $\mathrm{HPyV} 7$ in other hepatocellular diseases.

\section{Supplementary Materials}

The following are available online at https:/www.mdpi.com/2076-2607/8/8/1125/s1. Figure S1: (a) Agarose gel showing the specimen control size (SCS) ladder protocol to validate the DNA quality to be eligible for PCR testing. (b) Representative figure for validating the modified degenerated primer set by testing diverse PyVs positive control plasmids. Figure S2: $(\mathrm{a}, \mathrm{b})$ representative gel figures of DNA-PCR for HPyV7 targeting LTAg (98 bp) for the whole cohort. (c,d) A representative gel figure of DNA-PCR for $\mathrm{MCPyV}$ targeting the common region of LTAg and sTAg (M1/M2-178 bp). Figure S3: validating the pretreatment of FFPE and labelled probes for FISH. Figure S4: $(a, b)$ genome organization of HPyV6 627a (HM011563.1) showing the degenerated primers location in the LTAg (designed by genome compiler software). Table S1: morphometric analysis for HPyV7 IHC immunohistochemistry. Table S2: PCR-primers used in this study. 


\section{Author Contributions}

Conceptualization, F.K. and A.z.H.; methodology, F.K., G.M., E.C., E.J.M.S., and A.z.H.; investigation, F.K., G.M., D.R., and R.A.A.; data curation, F.K., G.M., and I.S.; writing—original draft preparation, F.K., G.M., and R.A.A.; writing—review and editing, A.z.H., V.W., and E.J.M.S., visualization, F.K., I.S., R.A.A., A.K.K., V.W., E.J.M.S., and E.C.; resources, D.R., S.W.O.D., U.P.N., and A.K.K.; principal investigator, supervision, and project administration, A.z.H. All authors have read and agreed to the published version of the manuscript.

\section{Funding}

This research received no external funding.

\section{Conflicts of Interest}

The authors declare no conflict of interest. 


\section{References}

1. Tyson, G.L.; El-Serag, H.B. Risk factors for cholangiocarcinoma. Hepatology 2011, 54, 173-184.

2. Gores, G.J. Cholangiocarcinoma: Current concepts and insights. Hepatology 2003, 37, 961-969.

3. Burak, K.; Angulo, P.; Pasha, T.M.; Egan, K.; Petz, J.; Lindor, K.D. Incidence and risk factors for cholangiocarcinoma in primary sclerosing cholangitis. Am. J. Gastroenterol. 2004, 99, 523-526.

4. Chan, J.F.; Tee, K.M.; Choi, G.K.; Zhu, Z.; Poon, R.W.; Ng, K.T.; Chanb, K.H.; Hungf, I.F.N.; Mane, K.; Yuen, K.Y. First detection and complete genome sequence of a phylogenetically distinct human polyomavirus 6 highly prevalent in human bile samples. J. Infect. 2017, 74, 50-59.

5. Gardner, S.D.; Field, A.M.; Coleman, D.V.; Hulme, B. New human papovavirus (B.K.) isolated from urine after renal transplantation. Lancet 1971, 1, 1253-1257.

6. $\quad$ Padgett, B.L.; Walker, D.L.; ZuRhein, G.M.; Eckroade, R.J.; Dessel, B.H. Cultivation of papova-like virus from human brain with progressive multifocal leucoencephalopathy. Lancet 1971, 1, 1257-1260.

7. DeCaprio, J.A.; Garcea, R.L. A cornucopia of human polyomaviruses. Nat. Rev. Microbiol. 2013, 11, 264-276.

8. Gheit, T.; Dutta, S.; Oliver, J.; Robitaille, A.; Hampras, S.S.; Combes, J.; Mckaychopin, S.; Le Calvezkelm, F.; Fenske, N.A.; Cherpelis, B.S.; et al. Isolation and characterization of a novel putative human polyomavirus. Virology 2017, 506, 45-54.

9. Bouvard, V.; Baan, R.; Grosse, Y.; Laubysecretan, B.; El Ghissassi, F.; Benbrahimtallaa, L.; Guha, N.; Straif, K. Carcinogenicity of malaria and of some polyomaviruses. Lancet Oncol. 2012, 13, 339-340.

10. Schowalter, R.M.; Pastrana, D.V.; Pumphrey, K.A.; Moyer, A.L.; Buck, C.B. Merkel cell polyomavirus and two previously unknown polyomaviruses are chronically shed from human skin. Cell Host Microbe 2010, 7, 509-515.

11. Feng, H.; Shuda, M.; Chang, Y.; Moore, P.S. Clonal integration of a polyomavirus in human Merkel cell carcinoma. Science 2008, 319, 1096-1100.

12. Jiang, M.; Abend, J.R.; Johnson, S.F.; Imperiale, M.J. The role of polyomaviruses in human disease. Virology 2009, 384, 266-273.

13. Dalianis, T.; Hirsch, H.H. Human polyomaviruses in disease and cancer. Virology 2013, 437, 63-72.

14. Federation of Dutch Medical Scientific Societies. Human Tissue and Medical Research: Code of Conduct for Responsible Use (2011); Federa: Rotterdam, The Netherlands, 2011.

15. Rennspiess, D.; Pujari, S.; Keijzers, M.; Abdul-Hamid, M.A.; Hochstenbag, M.; Dingemans, A.M.; Kurz, A.K.; Speel, E.J.; Haugg, A.; Pastrana, D.V.; et al. Detection of Human Polyomavirus 7 in Human Thymic Epithelial Tumors. J. Thorac. Oncol. 2015, 10, 360-366.

16. van Dongen, J.J.; Langerak, A.W.; Bruggemann, M.; Evans, P.A.; Hummel, M.; Lavender, F.L.; Delabesse, E.; Davi, F.; Schuuring, E.; Garcia-Sanz, R.; et al. Design and standardization of PCR primers and protocols for detection of clonal immunoglobulin and T-cell receptor gene recombinations in suspect lymphoproliferations: Report of the BIOMED-2 Concerted Action BMH4CT98-3936. Leukemia 2003, 17, 2257-2317. 
17. Haugg, A.M.; Rennspiess, D.; Hausen, A.Z.; Speel, E.J.; Cathomas, G.; Becker, J.C.; Schrama, D. Fluorescence in situ hybridization and qPCR to detect Merkel cell polyomavirus physical status and load in Merkel cell carcinomas. Int. J. Cancer 2014.

18. Beckervordersandforth, J.; Pujari, S.; Rennspiess, D.; Speel, E.J.; Winnepenninckx, V.; Diaz, C.; Weyers, W.; Haugg, A.M.; Kurz, A.K.; Zur Hausen, A. Frequent detection of human polyomavirus 6 in keratoacanthomas. Diagn. Pathol. 2016, 11, 58.

19. Hopman, A.H.; Kamps, M.A.; Smedts, F.; Speel, E.J.; Herrington, C.S.; Ramaekers, F.C. HPV in situ hybridization: Impact of different protocols on the detection of integrated HPV. Int. J. Cancer 2005, 115, 419-428.

20. Hafkamp, H.C.; Manni, J.J.; Haesevoets, A.; Voogd, A.C.; Schepers, M.; Bot, F.J.; Hopman, A.H.; Ramaekers, F.C.; Speel, E.J. Marked differences in survival rate between smokers and nonsmokers with HPV 16-associated tonsillar carcinomas. Int. J. Cancer 2008, 122, 2656-2664.

21. Anderson, C.M.; Zhang, B.; Miller, M.; Butko, E.; Wu, X.; Laver, T.; Kernag, C.; Kim, J.; Luo, Y.; Lamparski, H.; et al. Fully Automated RNAscope In Situ Hybridization Assays for Formalin-Fixed Paraffin-Embedded Cells and Tissues. J. Cell. Biochem. 2016, 117, 2201-2208.

22. Buck, C.B. Antibodies Used by the Buck Lab; NCI: Bethesda, MD, USA, 2019.

23. Moshiri, A.S.; Doumani, R.; Yelistratova, L.; Blom, A.; Lachance, K.; Shinohara, M.M.; Delaney, M.; Chang, O.; McArdle, S.; Thomas, H.; et al. Polyomavirus-Negative Merkel Cell Carcinoma: A More Aggressive Subtype Based on Analysis of 282 Cases Using Multimodal Tumor Virus Detection. J. Investig. Dermatol. 2017, 137, 819-827.

24. Hillen, L.M.; Rennspiess, D.; Speel, E.J.; Haugg, A.M.; Winnepenninckx, V.; Zur Hausen, A. Detection of Merkel Cell Polyomavirus in Seborrheic Keratosis. Front. Microbiol. 2017, 8, 2648.

25. Kuhn, M. Building Predictive Models in R Using the caret Package. J. Stat. Softw. 2008, 28, 1-26.

26. Harrell, F.E., Jr. Package 'rms'; Vanderbilt University: Nashville, TN, USA, 2018; Volume 2018, p. 229.

27. Kassem, A.; Schopflin, A.; Diaz, C.; Weyers, W.; Stickeler, E.; Werner, M.; Hausen, A.Z. Frequent detection of Merkel cell polyomavirus in human Merkel cell carcinomas and identification of a unique deletion in the VP1 gene. Cancer Res. 2008, 68, 5009-5013.

28. Duncavage, E.J.; Pfeifer, J.D. Human polyomaviruses 6 and 7 are not detectable in Merkel cell polyomavirus-negative Merkel cell carcinoma. J. Cutan. Pathol. 2011, 38, 790-796.

29. Nicol, J.T.J.; Robinot, R.; Carpentier, A.; Carandina, G.; Mazzoni, E.; Tognon, M.; Touze, A.; Coursaget, P. Age-specific seroprevalences of merkel cell polyomavirus, human polyomaviruses 6 , 7, and 9, and trichodysplasia spinulosa-associated polyomavirus. Clin. Vaccine Immunol. 2013, 20, 363-368.

30. Sroller, V.; Hamsikova, E.; Ludvikova, V.; Musil, J.; Nemeckova, S.; Salakova, M. Seroprevalence rates of HPyV6, HPyV7, TSPyV, HPyV9, MWPyV and KIPyV polyomaviruses among the healthy blood donors. J. Med. Virol. 2016, 88, 1254-1261.

31. Van der Meijden, E.; Bialasiewicz, S.; Rockett, R.J.; Tozer, S.J.; Sloots, T.P.; Feltkamp, M.C. Different serologic behavior of MCPyV, TSPyV, HPyV6, HPyV7 and HPyV9 polyomaviruses found on the skin. PLoS ONE 2013, 8, e81078. 
32. Kamminga, S.; van der Meijden, E.; Wunderink, H.F.; Touze, A.; Zaaijer, H.L.; Feltkamp, M.C.W. Development and Evaluation of a Broad Bead-Based Multiplex Immunoassay To Measure IgG Seroreactivity against Human Polyomaviruses. J. Clin. Microbiol. 2018, 56.

33. Kamminga, S.; Meijden, E.; Brouwer, C.; Feltkamp, M.; Zaaijer, H. Prevalence of DNA of fourteen human polyomaviruses determined in blood donors. Transfusion 2019, 59, 3689-3697.

34. Banales, J.M.; Marin, J.J.G.; Lamarca, A.; Rodrigues, P.M.; Khan, S.A.; Roberts, L.R.; Cardinale, V.; Carpino, G.; Andersen, J.B.; Braconi, C.; et al. Cholangiocarcinoma 2020: The next horizon in mechanisms and management. Nat. Rev. Gastroenterol. Hepatol. 2020.

35. Komuta, M.; Govaere, O.; Vandecaveye, V.; Akiba, J.; van Steenbergen, W.; Verslype, C.; Laleman, W.; Pirenne, J.; Aerts, R.; Yano, H.; et al. Histological diversity in cholangiocellular carcinoma reflects the different cholangiocyte phenotypes. Hepatology 2012, 55, 1876-1888.

36. Loyo, M.; Guerreropreston, R.; Brait, M.; Hoque, M.O.; Chuang, A.; Kim, M.S.; Sharma, R.; Liegeois, N.J.; Koch, W.M.; Califano, J.A.; et al. Quantitative detection of Merkel cell virus in human tissues and possible mode of transmission. Int. J. Cancer 2010, 126, 2991-2996.

37. Korup, S.; Rietscher, J.; Calvignac-Spencer, S.; Trusch, F.; Hofmann, J.; Moens, U.; Sauer, I.; Voigt, S.; Schmuck, R. Bernhard Ehlers Identification of a novel human polyomavirus in organs of the gastrointestinal tract. PLoS ONE 2013, 8, e58021.

38. van Beek, J.; Zur Hausen, A.; Klein Kranenbarg, E.; van de Velde, C.J.; Middeldorp, J.M.; van den Brule, A.J.; Meijer, C.J.; Bloemena, E. EBV-positive gastric adenocarcinomas: A distinct clinicopathologic entity with a low frequency of lymph node involvement. J. Clin. Oncol. Off. J. Am. Soc. Clin. Oncol. 2004, 22, 664-670.

39. Reimers, N.; Kasper, H.U.; Weissenborn, S.J.; Stützer, H.; Preuss, S.F.; Hoffmann, T.K.; Speel, E.J.; Dienes, H.P.; Pfister, H.J.; Guntinas-Lichius, O.; et al. Combined analysis of HPV-DNA, p16 and EGFR expression to predict prognosis in oropharyngeal cancer. Int. J. Cancer 2007, 120, 1731-1738.

40. Rozenblatt-Rosen, O.; Deo, R.C.; Padi, M.; Adelmant, G.; Calderwood, M.A.; Rolland, T.; Grace, M.; Dricot, A.; Askenazi, M.; Tavares, M.; et al. Interpreting cancer genomes using systematic host network perturbations by tumour virus proteins. Nature 2012, 487, 491-495.

41. Shuda, M.; Feng, H.; Kwun, H.J.; Rosen, S.T.; Gjoerup, O.; Moore, P.S.; Chang, Y. T antigen mutations are a human tumor-specific signature for Merkel cell polyomavirus. Proc. Natl. Acad. Sci. USA 2008, 105, 16272-16277. 


\section{Supplementary:}

a

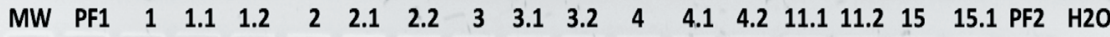
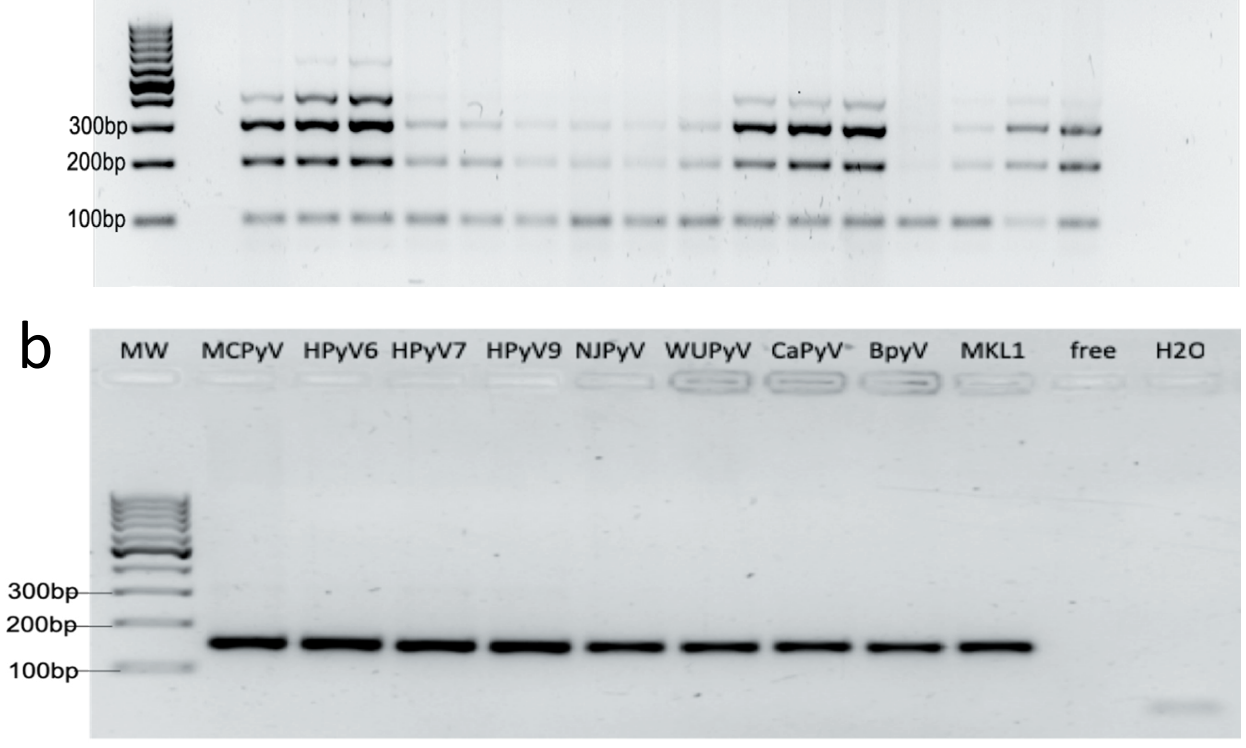

Figure S1. (a) Agarose gel showing the specimen control size (SCS) ladder protocol to validate the DNA quality to be eligible for PCR testing. (b) Representative images of DNA agarose gels for validating the modified degenerated primer set by testing diverse PyVs positive control plasmids and MKL1 cell line (142 to $158 \mathrm{bp}$ PCR products). Abbreviations: $\mathrm{H}_{2} \mathrm{O}$; water non-template negative control, PF; free paraffin without tissue, MW; molecular weight marker, MKL1; Merkel cell carcinoma cell line positive for MCPyV, Free; no master mix added to the gel. 


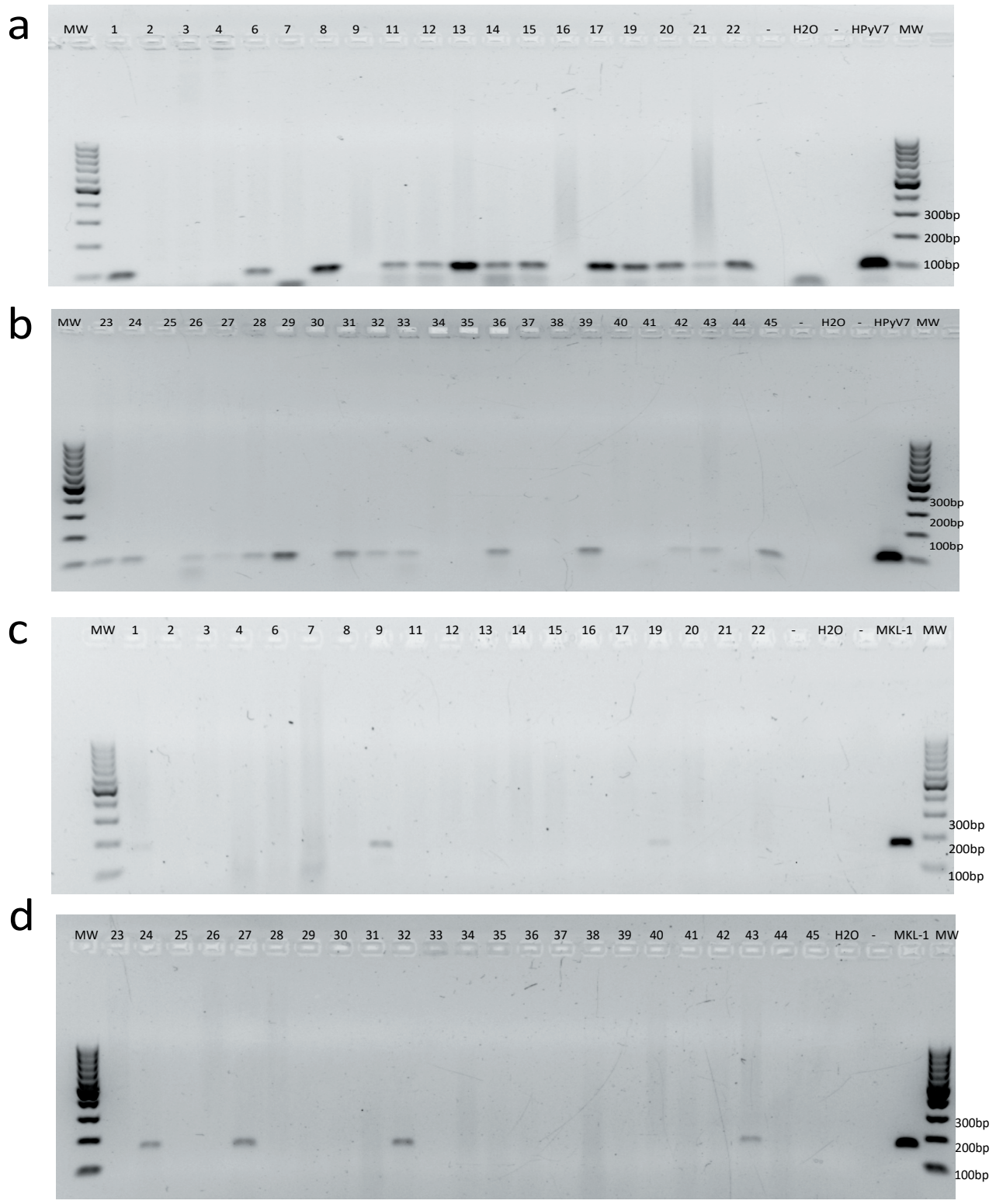

Figure S2. (a \& b) A representative gel figures of DNA-PCR for HPyV7 targeting LTAg (98bp). (c \& d) A representative gel figures of DNA-PCR for MCPyV targeting the common region of LTAg and sTAg (M1/ M2-178bp). Abbreviations: $\mathrm{H}_{2} \mathrm{O}$; water non-template negative control, MW; molecular weight marker, $\mathrm{HPyV7}$; Human polyoma virus, MKL1; Merkel cell carcinoma cell line positive for MCPyV as a positive control,-; no master mix added to the gel slot. 

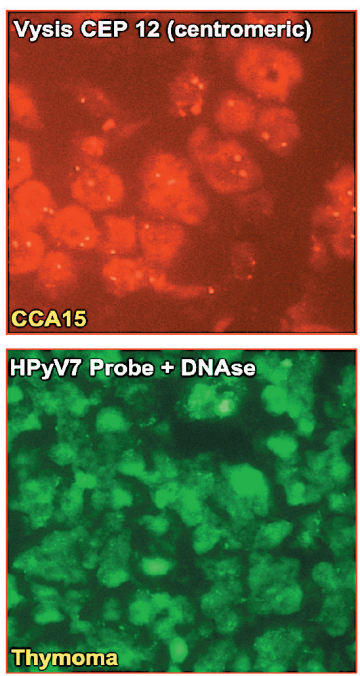

b
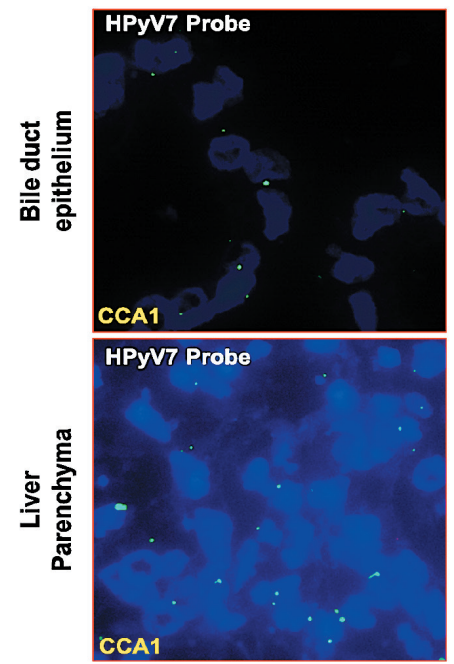
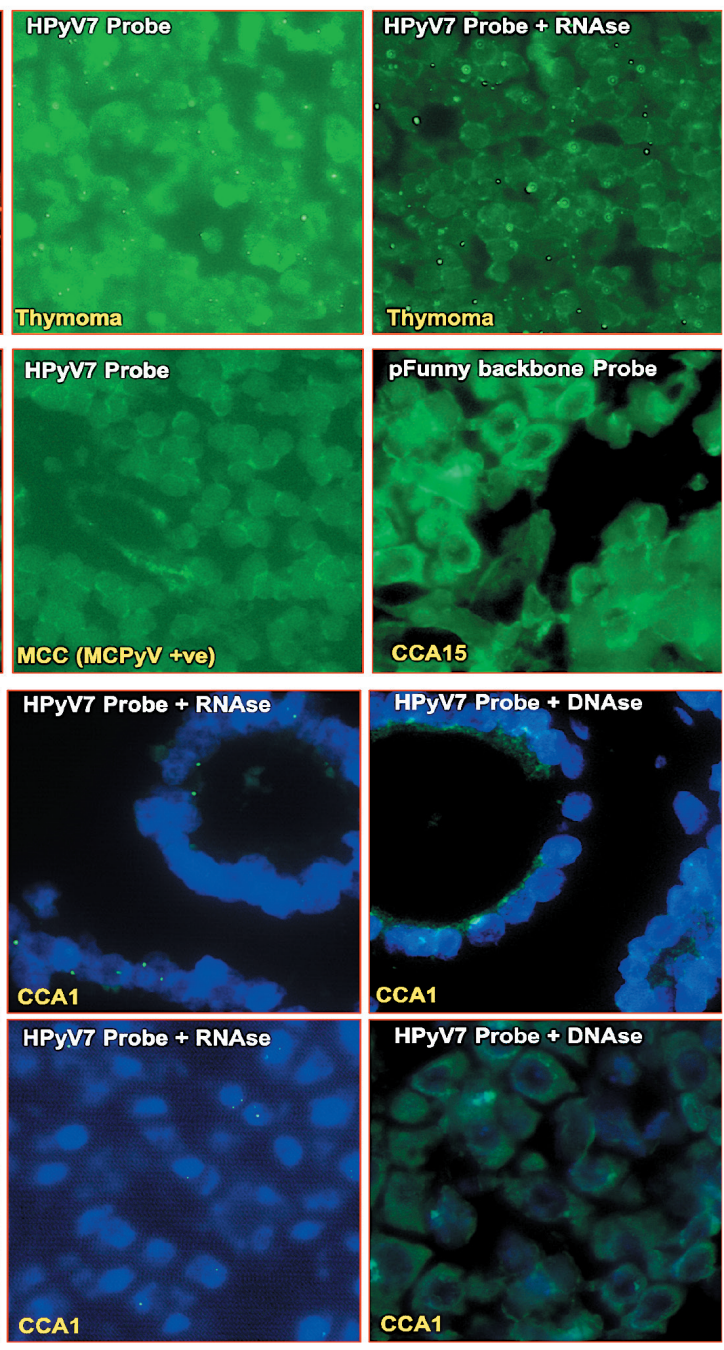

Figure S3. Validating the pretreatment of FFPE and labelled probes for FISH. (a) Validating the DNA quality and integrity for subsequent HPyV FISH analyses. Using the CEP probe directed against alpha satellite DNA located at the centromere of chromosome 12 (orange). Two copies of each signal were observed in the nuclei all parenchymal and non-parenchymal liver cells. Validating the whole HPyV7 genome probe labeling, specific green punctate dot FISH signals in thymic cells which used as control for HPyV7. HPyV7 Probe with RNase for thymoma tissues section demonstrate a slightly diminished of the fluorescence signal dots. Treatment the thymoma tissue with DNAse I in combination with the HPyV7 probe erased the signals completely. Merkel cell carcinoma tissue with positive MCPyV tested with HPyV7 probe to exclude the cross reactivity of the labeled probe with $\mathrm{MCPyV}$. Testing the labeled pFunny backbone probe which used as backbone for HPyV7 plasmid and revealed no signals in the CCA and non-neoplastic hepatocyte cells which were positive for $\mathrm{HPyV} 7$ probe FISH. (b) Representative images for validating FISH for the HPyV7 probe using CCA tissue positive for HPyV7 by PCR, specific green punctate dot FISH signals in CCA and hepatic cells representing HPyV7 DNA. The signals were slightly decreased after RNase A treatment, however using DNase I erased the signals completely. 


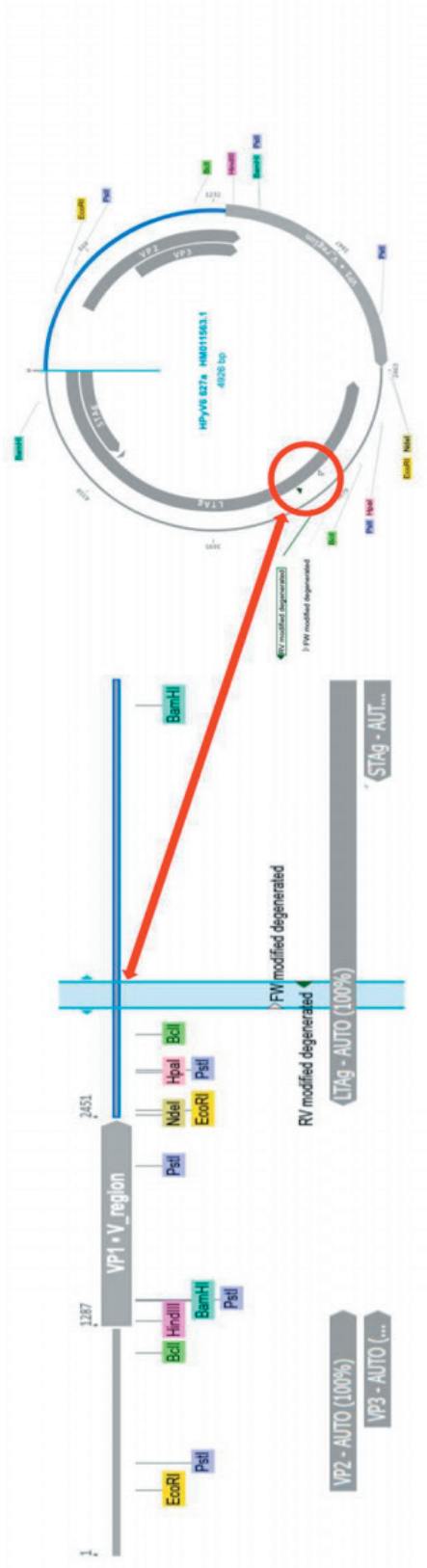

$\dddot{乛}$

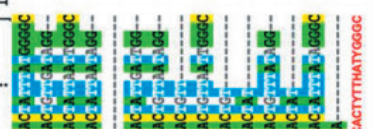

Jँ

·

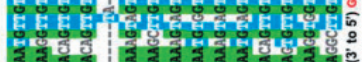

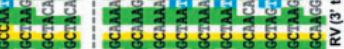

내의

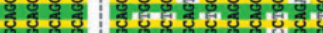

H.

둥탱

ชुํํำ

어의.

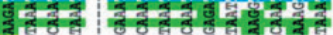

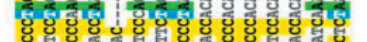

- $\rightarrow$ o g

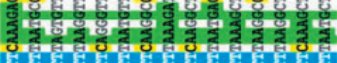

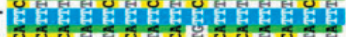

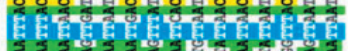

.

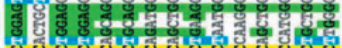

- हु

E

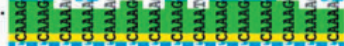

.. छั

는ㄷㅇㄴ

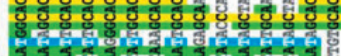

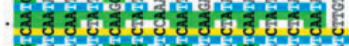

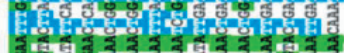

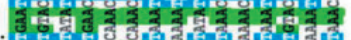

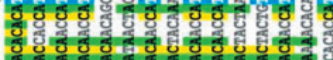

बू.

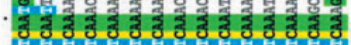

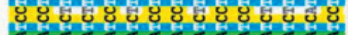

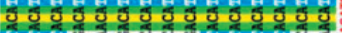

- E

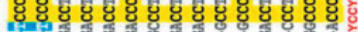

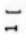

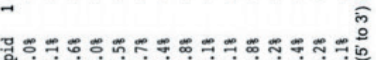

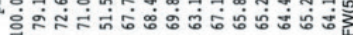

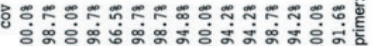

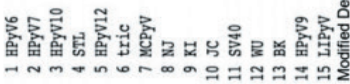

$\nabla$

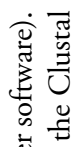

离合泀

8.5

है 造

品密

ล․․ㄹ

훙

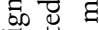

氙苛

造过

㭋

\pm 동

3.

ठ

O

的

ฮั छี

ह 둰

동

跑泀离

잉ㅇㅇ 군

过

ฐ

我泀

क

年

هัర

ஏ士

坖范

ㄷำ

तु

ป ह

在

I

0 車

ㅇำ

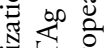

블

댕ㄴㄷㅇ

亖

ह

ญิ

() ปั.

फं

可

总合 
Supplementary Table 1: Morphometric analysis for HPyV7 IHC immunohistochemistry

\begin{tabular}{llll}
\hline \multirow{2}{*}{ Patient ID } & \multicolumn{2}{l}{ HPyV7 IHC } \\
\cline { 2 - 4 } & \multicolumn{2}{l}{ Average of positive cells by HPF } \\
\cline { 2 - 4 } & Hepatocyte & $\begin{array}{l}\text { Bile duct } \\
\text { epithelium }\end{array}$ & CCA \\
\hline CCA1 & 23 & 18 & 11 \\
\hline CCA6 & 3 & 11 & - \\
\hline CCA8 & 6 & 10 & 26 \\
\hline CCA11 & 13 & 13 & 21 \\
\hline CCA12 & 4 & 12 & 2 \\
\hline CCA13 & - & - & 8 \\
\hline CCA14 & 52 & 8 & - \\
\hline CCA15 & 40 & 2 & 40 \\
\hline CCA17 & 1 & 1 & - \\
\hline CCA19 & 2 & 2 & 2 \\
\hline CCA20 & 2 & 4 & 3 \\
\hline CCA21 & 7 & 3 & 3 \\
\hline
\end{tabular}

Abbreviations: Patient ID, lab identification number; CCA, Cholangiocarcinoma; HPyV7, human polyomavirus 7; IHC, immunohistochemistry; HPF, high power field 


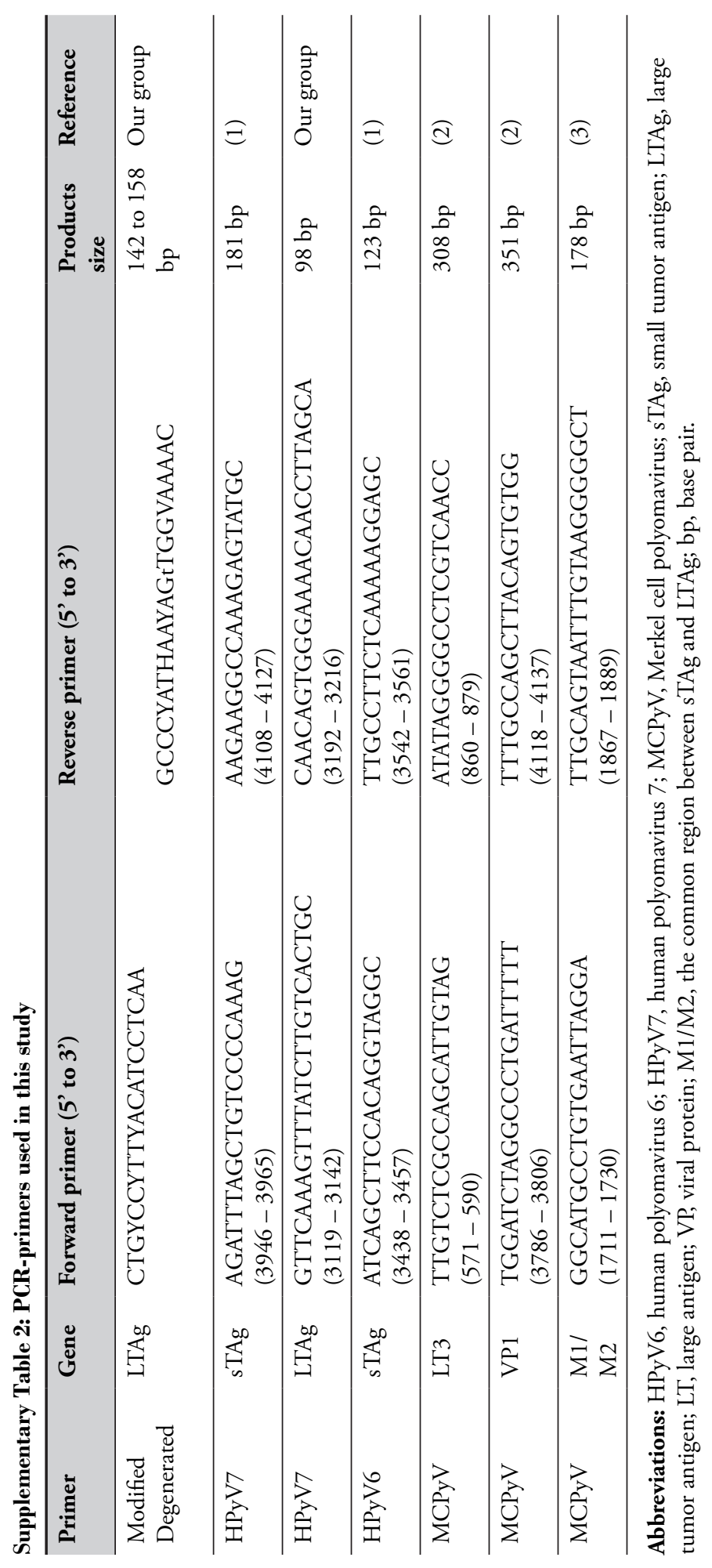




\section{References:}

1. Schowalter RM, Pastrana DV, Pumphrey KA, Moyer AL, Buck CB. Merkel cell polyomavirus and two previously unknown polyomaviruses are chronically shed from human skin. Cell host $\&$ microbe. 2010;7(6):509-15.

2. Feng H, Shuda M, Chang Y, Moore PS. Clonal integration of a polyomavirus in human Merkel cell carcinoma. Science (New York, NY). 2008;319(5866):1096-100.

3. Kassem A, Schopflin A, Diaz C, Weyers W, Stickeler E, Werner M, et al. Frequent detection of Merkel cell polyomavirus in human Merkel cell carcinomas and identification of a unique deletion in the VP1 gene. Cancer research. 2008;68(13):5009-13. 


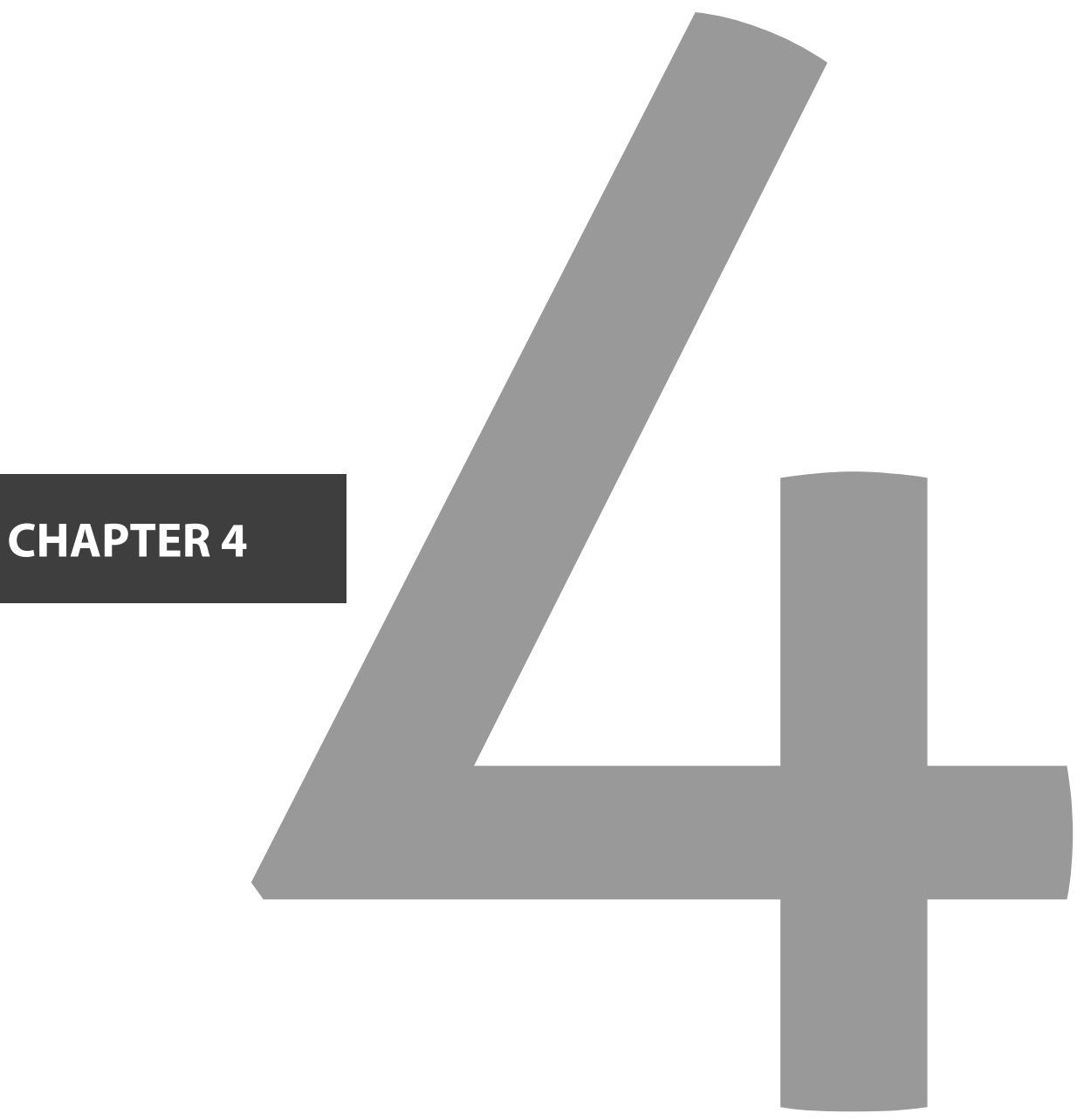




\section{Reactivation of BK Polyomavirus in Urine Cytology is Not Associated with Urothelial Cell Carcinoma}

Faisal Klufah, Ghalib Mobaraki, Axel zur Hausen, and Iryna V. Samarska 


\section{ABSTRACT}

BK polyomavirus (BKPyV) has been associated with some high-grade and special urothelial cell carcinoma (UCC) subtypes in immunosuppressed patients. Here, we evaluated the relationship of BKPyV-positive urine cytology specimens (UCS) with UCC. A large single-institution database was retrospectively searched for UCS positive for decoy cells, suggesting BKPyV infection. These were tested for the presence of BKPyV by PCR and immunohistochemistry (IHC) in urine sediments and formalin-fixed paraffinembedded (FFPE) tissue samples of UCC. Decoy cells were reported in 30 patients out of the database with 22.867 UCS. Of these 30 patients, 16 (53.3\%) had no history of UCC. Six patients out of these 16 had a history of transplantation, 4 had a history of severe chronic medical conditions, and 6 had no chronic disease. The other fourteen patients were diagnosed with either in situ or invasive UCC of the urinary bladder $(14 / 30 ; 46.6 \%)$ prior to the detection of decoy cells in the urine. Nine of these UCC patients received intravesical treatment (BCG or mitomycin) after the first presentation with UCC. However, the clinical data on the treatment of the other five UCC patients was lacking. IHC identified BKPyV-positivity in the urine samples of non-UCC and UCC patients, while no BKPyV positivity was found in FFPE tissues of primary UCCs and metastases. In addition, BKPyV-PCR results revealed the presence of BKPyV DNA in the urine of the UCC cases, yet none in the UCC tissues itself. These data strongly indicate that $\mathrm{BKPyV}$ reactivation is not restricted to immunosuppression. It can be found in UCS of the immunocompetent patients and may be related to the intravesical BCG or mitomycin treatment of the UCC patients.

Keywords: BKPyV; small DNA viruses; bladder cancer; cancer; polyomavirus; tumorigenesis; decoy cells 


\section{INTRODUCTION}

BK polyomavirus (BKPyV) is a human polyomavirus that has been suspected as a putative oncogenic virus in the development of urothelial cell carcinomas (UCC) in immunocompromised patients ${ }^{1,2}$. Approximately $90 \%$ of the human population is infected during early life with BKPyV. The virus remains latent in the epithelium of the proximal tubule of the kidney, within the urothelial cell layer of the bladder, ureters, renal pelvis, and other tissue types ${ }^{1}$. BKPyV reactivation frequently occurs following immunosuppression, especially in the context of kidney and solid organ transplantations, with the risk of developing BKPyV-associated nephropathy (PyVAN) ${ }^{3,4}$. BKPyV replication has been described to be a significant risk factor for bladder cancer development following kidney transplantation ${ }^{2,5}$. Thus, BKPyV-positivity has been reported previously in the urinary bladder and kidney tumors occurring after PyVAN $2,5,6,7,8,9,10$. The spectrum of the BKPyV positive urinary bladder lesions includes high-grade urothelial carcinoma (UCC; both in situ and invasive), micropapillary urothelial carcinoma, pleomorphic giant cell carcinoma, bladder adenocarcinoma, and nephrogenic adenoma $35,6,7,8,9$. Moreover, BKPyV-DNA was detected in many other tumors, including pancreas, liver, oral, oropharyngeal, laryngeal squamous cell carcinomas, rhabdomyosarcoma, Kaposi's sarcoma, prostate adenocarcinoma, and brain tumors ${ }^{2,10,11}$. In 2012, the International Agency for Research on Cancer (IARC) classified $\mathrm{BKPyV}$ as a group $2 \mathrm{~B}$ possibly carcinogenic candidate to humans ${ }^{2}$.

BKPyV-infected cells from renal tubules and urothelium can be detected by cytology as decoy cells that have enlarged and altered nuclei with large homogeneous basophilic nuclear inclusions, which mimic cellular changes observed in UCC in situ ${ }^{3,12}$. The identification of BKPyV-IHC positive decoy cells in voided urine has thus been interpreted as a strong indicator of $\mathrm{BKPyV}$ reactivation in urothelial cells following immunosuppressive treatment ${ }^{3,4}$. These cells have been shown to express BKPyV large $\mathrm{T}$ antigen (LTAg) using immunohistochemistry (IHC) ${ }^{7}$. Due to its known cross-reactivity with the LTAg of BKPyV, an anti-Simian virus 40 (SV40) LTAg antibody is used in clinical practice as a surrogate marker of $\mathrm{BKPyV}$ infection ${ }^{1,13}$.

The aim of this study was to assess the relation of BKPyV-positive urine cytology specimens (UCS) to the detection of UCC in a large UCS database and the following evaluation of BKPyV in the UCC of the urinary bladder. In addition, we aimed to evaluate the relation of $\mathrm{BKPyV}$ to intravesicular BCG or mitomycin treatment of UCC patients. 


\section{MATERIALS AND METHODS}

\subsection{Study Population}

The starting point of this study was a single-institution database that included 22.867 UCS. This database was searched for UCS containing decoy cells as detected by cytology over a 15 year period (January 2004-December 2019) using the laboratory information system of the department of pathology, Maastricht University Medical Center+ (MUMC+), the Netherlands. Thirty patients were identified and included in this study for further workup and analyses. All cytology slides, including immunocytochemistry for SV40, were retrieved and independently reviewed by two pathologists (IVS and AzH). Cases that also had bladder biopsies or resections obtained following and preceding the urinary cytology specimen were collected from the archive and independently reviewed. Clinical information regarding medical history, transplantation, chronic medical condition (autoimmune disease, cancer, diabetes, immunosuppressive treatment, chemotherapy, radiotherapy, and connective tissue disease), as well as the history of renal and bladder tumors were obtained from the medical records of the MUMC+. This study was approved by the Medical Ethics Review Committee of the Maastricht University Medical Center in the Netherlands (2019-0977). All specimens were collected and studied in accordance with the protocol of the Dutch Code of Conduct for Observational Research with Personal Data (2004) and Tissue ${ }^{14}$.

\subsection{Cytology}

Unfixed urine samples were prepared by the cytospin method, according to the previously described protocol ${ }^{15}$. One cytospin was stained with Papanicolaou stain, screened by a cytotechnologist, and subsequently reviewed and reported by a pathologist. The other cytospin was stained with the immunoperoxidase staining for SV40 LTAg immunocytochemistry (ICC). The ICC was performed using SV40 LTAg antibody (clone: PAb416, dilution 1:500, Calbiochem Inc., San Diego, CA, USA) with a Dako's autostainer Link 48 using the EnVision FLEX visualization kit (K8008, DAKO, Carpinteria, CA, USA) according to the diagnostic standard routine and manufacturer's protocol. Only nuclear staining was regarded as a positive reaction for the SV40 antibody.

\subsection{Histology}

Urinary bladder biopsy and resection specimens were fixed in $4 \%$ buffered formalin and embedded in paraffin, according to the routine pathology diagnostic procedures. For light microscopy, hematoxylin and eosin ( $\mathrm{H} \& \mathrm{E})$-stained sections were performed on 3-5 $\mu$ m-thick tissue sections. 


\subsection{Immunohistochemistry}

Representative formalin-fixed paraffin-embedded tissues (FFPE) were chosen from bladder biopsy and resection cases, and IHC was performed using the SV40 LTAg antibody (clone: PAb416, dilution 1:500, Calbiochem Inc. San Diego, CA, USA). The IHC staining was performed on 3-5 $\mu \mathrm{m}$-thick FFPE sections using Dako's autostainer as described above in the cytology section. Only nuclear protein expression was interpreted as a positive reaction for the SV40 antibody. All slides were scanned by the VENTANA iScan-HT slide scanner (Roche Diagnostics Inc., Tucson, AZ, USA).

\subsection{BKPyV PCR}

Nine FFPE specimens and five urine sediments of UCC-patients were retrieved from the archive at the Department of Pathology, Maastricht University Medical Center+. DNA was extracted from all specimens using the protocol of genomic DNA isolation by (NucleoSpin ${ }^{\oplus}$ Tissue, Macherey-Nagel GmbH \& Co., Düren, Germany). The DNA concentration was assessed using a spectrophotometer (NanoDrop 2000, Thermo Scientific., Wilmington, DE, USA). Per sample, $125 \mathrm{ng}$ DNA were added to the PCR reaction. All isolated DNAs were assessed for quality and integrity using multiplex primers (SCS: specimen control size) as described previously ${ }^{16,17}$.

Two PCR primer sets were used to screen for the presence of BKPyV DNA. One primer set targeted the viral LTAg, while the other targeted the viral protein 1 (VP1), all primer sequences listed in Supplementary Table S3. Sequencing of all obtained PCR products was compared and analyzed with the reference sequences of the National Center for Biotechnology Information (NCBI), Entrez nucleotide database using the NCBI Blast program.

\section{RESULTS}

\subsection{Study Population and Urine Cytology}

Of the 22.867 retrospectively evaluated UCS, 46 urine specimens $(0.2 \%)$, obtained from 30 patients, were positive for decoy cells, indicating BKPyV-infection (Figure 1A). Six of these patients had two or more urine specimens with these viral changes. UCS were obtained from 25 males with a median age of 68.6 years (range 25-90) and 5 females with a median age of 55 years (range 38-80). All clinicopathological data of the patients are summarized in Table 1. The follow-up period ranged from 7 months to 170 months, with a median of 70.5 months following or preceding the urinary cytology. All 30 patients showed typical decoy cells in their urine specimens by cytology. These cells were characterized by an enlarged nucleus with an intranuclear inclusion, which has an amorphous basophilic ground-glass appearance (Figure 1). 
A
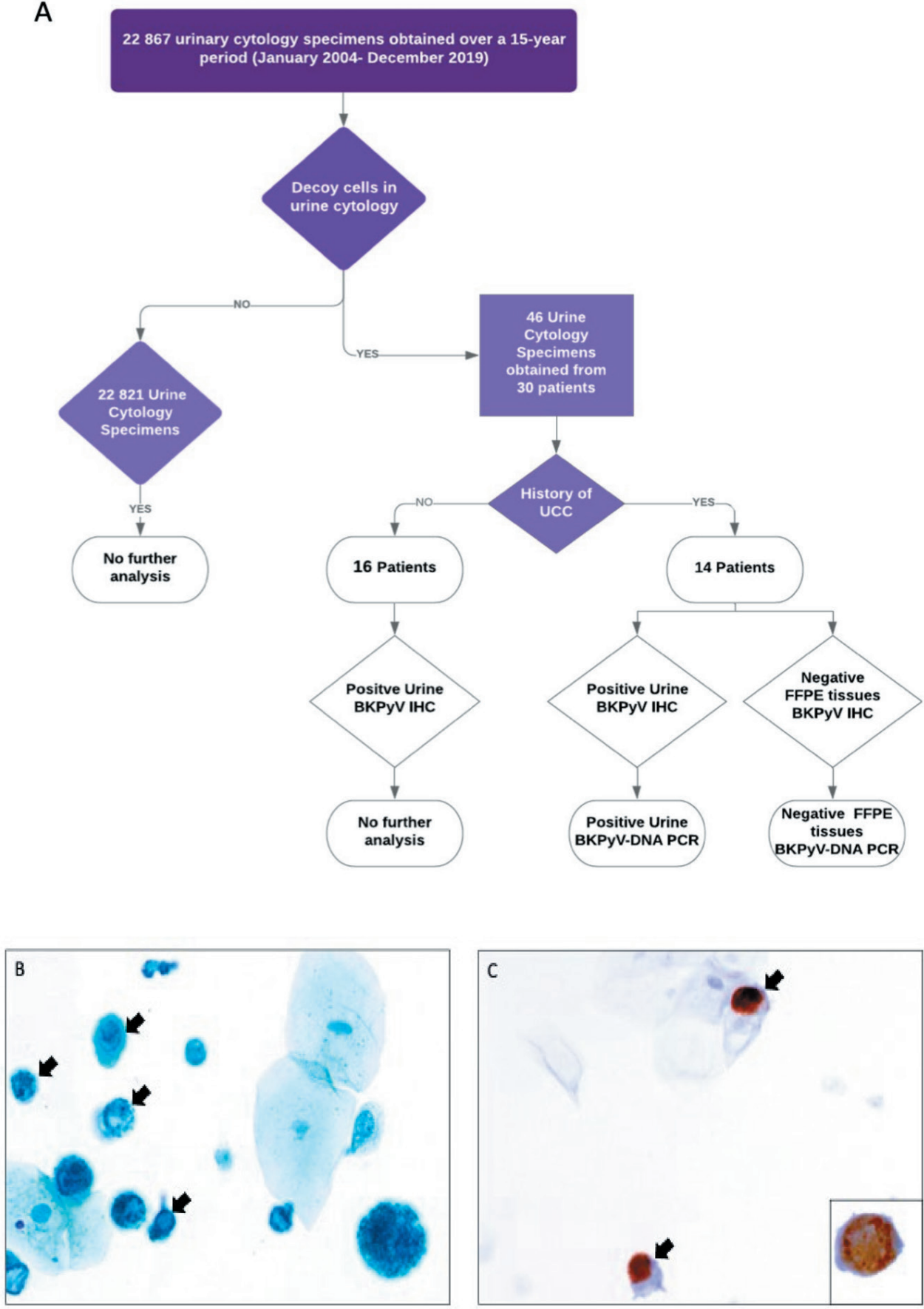

Figure 1. (A) Flow chart depicting the study design and immunohistochemistry results. (B) Decoy cells (arrows) in urine cytology (Papanicolaou staining) and (C) immunohistochemistry for BKPyV (arrows), and an enlarged image in the lower right corner showing the expression of BKPyV. The images were taken at $200 \times$ magnification. 
Table 1. Patients clinicopathological data, demographics, and results.

\begin{tabular}{|c|c|}
\hline Clinicopathological Characteristic & Patients $(n=30)$ \\
\hline Age range (years) & $25-90$ \\
\hline Male & $25-90$ \\
\hline Female & $38-80$ \\
\hline BKPyV-IHC+ve in urine cytology & $27 / 30$ \\
\hline Non-UCC patients & $13 / 16$ \\
\hline UCC-patients & $14 / 14$ \\
\hline Non-UCC patients & 16 \\
\hline Immunosuppressed & 10 \\
\hline Transplantation & 6 \\
\hline Renal & 5 \\
\hline Stem cell & 1 \\
\hline Chronic disease (IBD, AIH, CTD, other malignancy) & 4 \\
\hline No chronic disease & 6 \\
\hline UCC patients & 14 \\
\hline LGUCC & 6 \\
\hline HGUCC & 1 \\
\hline INUCC & 1 \\
\hline CIS & 2 \\
\hline LGUCC and HGUCC & 2 \\
\hline CIS and HGUCC & 1 \\
\hline CIS and INUCC & 1 \\
\hline UCC with treatment & 9 \\
\hline Intravesical treatment & 7 \\
\hline BCG & 4 \\
\hline Mitomycin & 2 \\
\hline Both BCG and mitomycin & 1 \\
\hline Radiotherapy & 1 \\
\hline Intravesical treatment and radiotherapy & 1 \\
\hline UCC with unknown treatment & 5 \\
\hline
\end{tabular}

UCC, urothelial cell carcinoma; IHC, immunohistochemistry; +ve: positive; IBD, inflammatory bowel disease; AIH, autoimmune hepatitis; CTD, connective tissue disease; LGUCC, low-grade non-invasive urothelial cell carcinoma; HGUCC, high-grade non-invasive urothelial cell carcinoma; CIS, carcinoma in situ; INUCC, invasive urothelial cell carcinoma; BCG, Bacillus Calmette-Guerin.

\subsection{BKPyV in Patients without a History of Urothelial Cell Carcinoma} Of the thirty patients, sixteen had no history of UCC (53.3\%). Out of the sixteen patients without a history of UCC, five patients $(5 / 16 ; 31.25 \%)$ had a history of kidney transplantation, and one patient $(1 / 16 ; 6.25 \%)$ had a history of stem cell transplantation. Other four patients $(4 / 16 ; 25 \%)$ from the non-UCC history group had a history of chronic diseases that required immunosuppression and systemic therapy/radiotherapy, 
such as ulcerative colitis, diabetic nephropathy, autoimmune thyroiditis, autoimmune hepatitis, and not-urological cancer (prostate adenocarcinoma, colon adenocarcinoma, high-grade sarcoma of the soft tissue). The other six patients $(6 / 16 ; 37.5 \%)$, who had follow-up history in the database for at least one year after the first presentation with $\mathrm{BKPyV}$ positive urine cytology, did not have any history of chronic diseases, nor were diagnosed with UCC during the follow-up period (Table 1 and Supplementary Table S1). Those six patients could be considered as immunocompetent for the period of their follow-up in our institution. Of the sixteen non-UCC patients, thirteen (81.25\%) were positive for BKPyV IHC in urinary samples (Figure 1C). IHC could not be performed in three patients because no material was left due to multiple usages for additional analyses (Table S1).

\subsection{Presence of BKPyV in Urothelial Cell Carcinoma}

All fourteen patients with a history of UCC developed cancer prior to the detection of BKPyV-IHC positive decoy cells in their urine. Decoy cells in their urine were detected in the follow-up after the first presentation with UCC with a median period of 3.5 years (range 1-13 years) and were positive for the SV40 LTAg by immunohistochemistry, indicating the BKPyV-reactivation in urothelial cells (Figure 1C; Table 2; Supplementary Table S2). Additionally, BKPyV-DNA PCR of the five available urine sediments revealed BKPyV-positivity as confirmed by sequencing (Table 2). Interestingly, none of these patients had decoy cells in urine by preoperative cytology prior to the diagnosis of UCC.

Nine of these patients $(9 / 14 ; 64.3 \%)$ were known to have a history of intravesical treatment, either BCG, mitomycin, or/and radiotherapy (Table 1). The treatment history of the other five UCC patients is lacking. Two patients had recurrent UCC after intravesical treatment, and the diagnosis of $\mathrm{BKPyV}$ infection was confirmed by cytology. Twelve patients $(12 / 14 ; 85.7 \%)$ presented with a conventional type UCC (either lowgrade, high-grade UCC, or CIS) and two patients had invasive UCC with sarcomatoid differentiation (Supplementary Table S2). No micropapillary urothelial carcinoma, pleomorphic giant cell carcinoma, or bladder adenocarcinomas were found in the patients' cohort. The H\&E-tained slides of all 14 UCC patients were examined histologically and revealed no virus-related cytopathic changes (Figure 2A). BKPyV-IHC was performed on the available UCC FFPE specimens (with either in situ or invasive UCC of the urinary bladder) of 12 patients (Table 2, Supplementary Table S2). BKPyV-IHC was negative in all tested UCC samples (Figure 2B, Table 2), including metastatic and recurrent UCC. In addition, BKPyV DNA PCR was performed on the available UCC FFPE tissues of the nine patients, and all nine samples were negative for BKPyV-DNA (Table 2). Due to multiple previous histopathological and immunochemical diagnostic procedures, no FFPE tissues of the remaining five UCCs were available to perform BKPyV-IHC or PCR (Table S2). 
Table 2. Clinical data, BKPyV IHC, and DNA PCR results.

\begin{tabular}{|c|c|c|c|c|c|c|c|c|}
\hline \multirow{3}{*}{$\begin{array}{l}\text { Patient } \\
\text { ID. }\end{array}$} & \multirow[t]{3}{*}{ Diagnosis } & \multirow{3}{*}{$\begin{array}{l}\text { (Intravesical) } \\
\text { Treatment }\end{array}$} & \multicolumn{2}{|c|}{ BKPyV IHC } & \multicolumn{4}{|c|}{ BKPyV PCR } \\
\hline & & & \multirow{2}{*}{$\begin{array}{l}\text { Urine } \\
\text { Cytology }\end{array}$} & \multirow{2}{*}{$\begin{array}{l}\text { FFPE } \\
\text { Tissue }\end{array}$} & \multicolumn{2}{|c|}{ Urine Sediments } & \multicolumn{2}{|c|}{ FFPE Tissue } \\
\hline & & & & & LTAg & VP1 & LTAg & VP1 \\
\hline I.7 & LGUCC & Mitomycin & + & n.a. & n.a. & n.a. & n.a. & n.a. \\
\hline I.8 & LGUCC & Unknown & + & - & n.a. & n.a. & n.a. & n.a. \\
\hline I.9 & LGUCC & Unknown & + & - & n.a. & n.a. & n.a. & n.a. \\
\hline I.10 & LGUCC, HGUCC & BCG/Mitomycin & + & - & n.a. & n.a. & - & - \\
\hline I.12 & INUCC & Unknown & + & n.a. & n.a. & n.a. & n.a. & n.a. \\
\hline I.13 & CIS, INUCC & BCG/radiotherapy & + & - & n.a. & n.a. & - & - \\
\hline I.15 & LGUCC & Unknown & + & - & n.a. & n.a. & - & - \\
\hline I.17 & CIS & BCG & + & - & n.a. & n.a. & - & - \\
\hline I.21 & HGUCC & Radiotherapy & + & - & n.a. & n.a. & - & - \\
\hline I.22 & CIS & BCG & + & - & + & - & - & - \\
\hline I.23 & LGUCC, HGUCC & BCG & + & - & + & - & - & - \\
\hline I.26 & CIS and HGUCC & BCG & + & - & + & + & - & - \\
\hline I.29 & LGUCC & Mitomycin & + & - & + & - & n.a. & n.a. \\
\hline I.30 & LGUCC & Unknown & + & - & + & - & - & - \\
\hline
\end{tabular}

Patient ID, lab identification number; FFPE, formalin-fixed paraffin-embedded tissues; UCC, urothelial cell carcinomas; PCR, polymerase chain reaction; +, positive; -, negative; LTAg, large tumor antigen; VP1, viral protein; UCC, urothelial cell carcinoma; n.a., not applicable; IHC, immunohistochemistry; LGUCC, low-grade non-invasive urothelial cell carcinoma; HGUCC, high-grade non-invasive urothelial cell carcinoma; INUCC, invasive urothelial cell carcinoma; CIS, carcinoma in situ; BCG, Bacillus Calmette-Guerin.

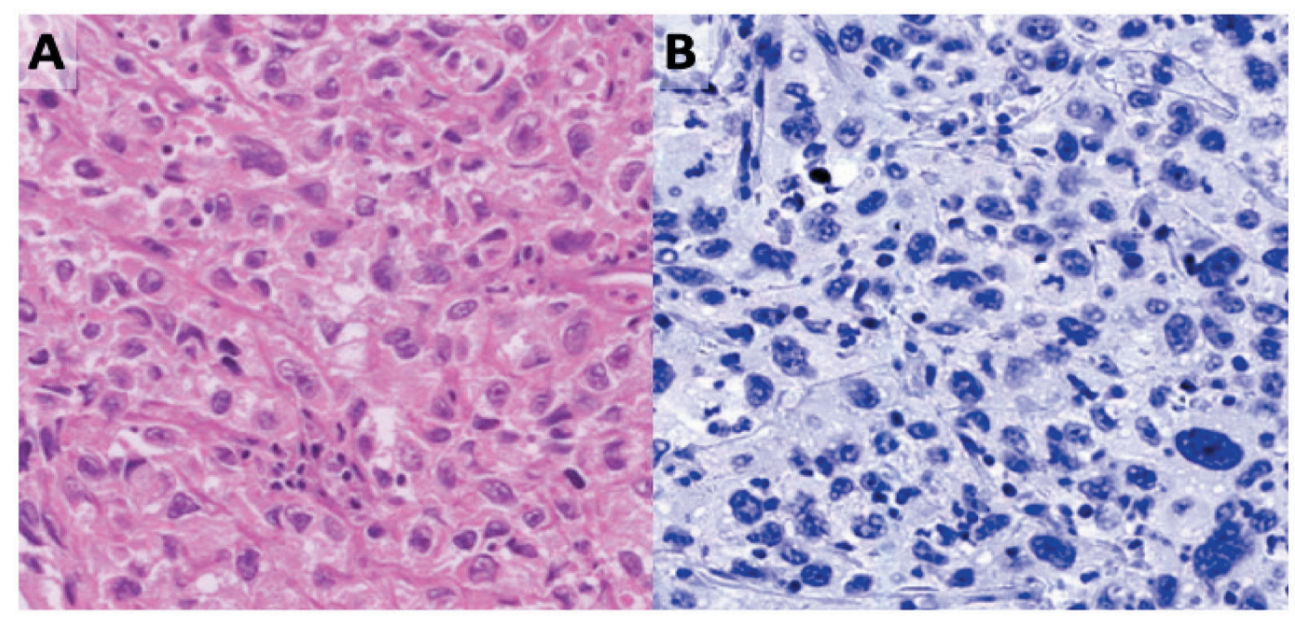

Figure 2. High-grade urothelial cell carcinoma and immunohistochemistry for BKPyV. (A) H\&E-stained section shows neoplastic UCC cells with no visible viral changes in the nuclei. (B) a representative BKPyVnegative UCC as tested by IHC (200× magnification). 


\section{DISCUSSION}

In this study, we evaluated the association of BKPyV-positive urine cytology with UCC and the presence of BKPyV in UCS and FFPE samples of the UCC cohort. The overall cytology results revealed a very low percentage $(0.2 \%)$ of BKPyV-positive urine cytology in the database of our institution over a 15 year period (January 2004-December 2019). Several studies reported that decoy cells were detected in 18 to $28 \%$ of the urine sediments of patients with kidney transplantation $18,19,20,21,22,23$. In contrast to those studies, our study was not restricted to immunocompromised patients but also included patients with no previous history of organ transplantation, chemotherapy, radiotherapy, malignancy, or chronic disease, requiring immunosuppressive therapy (such as colitis ulcerosa, autoimmune disease, diabetes, connective tissue disease). Previous studies reported similar low detection rates of BKPyV in UCS when all patients (immunocompromised and immunocompetent) were included in the search ${ }^{12,24}$.

The main finding of our work is that BKPyV was found in the UCS of the patients diagnosed with UCC and was detected in the follow-up period after the initial diagnosis of the UCC was made. Moreover, this BKPyV-infection may be related to the intravesical treatment of their UCC. In our study, UCC developed before the diagnosis of BKPyVinfection in urine cytology and were negative for BKPyV-IHC or PCR in FFPE tissue samples.

BKPyV has been associated with some types of UCC in the context of immunosuppression, polyomavirus-associated nephropathy (PVAN), and hemorrhagic cystitis 1,2,25,26. The asymptomatic urinary shedding of $\mathrm{BKPyV}$ has been reported in healthy immunocompetent individuals as well ${ }^{27}$. It is known that $\mathrm{BKPyV}$ remains latent following the infection of the urinary tract in the proximal tubular epithelium of the kidney, the urothelial cell layer of the bladder, ureters, or renal pelvis ${ }^{1}$. Immunosuppression due to any clinical reason leads to the reactivation of $\mathrm{BKPyV}$ from viral latency ${ }^{28,29}$. The detection of decoy cells in voided urine is a widely used diagnostic tool as a cytopathological surrogate marker of a BKPyV-infection ${ }^{30}$ and has several clinical implications. These cells mimic atypical neoplastic cells and can be misdiagnosed as carcinoma in situ ${ }^{12}$. The reactivation of $\mathrm{BKPyV}$ can lead to BKPyV-associated nephropathy. BKPyV-associated nephropathy develops in $1-10 \%$ of kidney transplant recipients and can ultimately result in renal graft loss in $30 \%$ up to $80 \%$ of the cases ${ }^{25,28}$.

The prevalence of $\mathrm{BKPyV}$ varies from one study to another, which probably depends on the number of transplantations performed in the corresponding institution. Decoy cells are more often found in patients with a history of kidney or stem cell transplantation ${ }^{25,28}$. Our results show that BKPyV-positive decoy cells in voided urine samples were 
also detected in patients with chronic diseases, such as autoimmune diseases, diabetes, ulcerative colitis, diabetic nephropathy, and non-urological cancer, which could be explained by immunosuppressive or systemic therapy. Our findings are in line with previously reported results by Reploeg et al. ${ }^{31}$.

Interestingly, $20 \%$ of the patients in our cohort did not have any history of immunosuppression, transplantation, or cancer, which could explain the reactivation of BKPyV. Thus, our data show that BKPyV-infection can occur in immunocompetent patients who did not have any history of UCC, organ transplantation, autoimmune or immunomodulated disease, or immunosuppressive medication. The reactivation of $\mathrm{BKPyV}$ was reported previously in immunocompetent patients with hemorrhagic and non-hemorrhagic cystitis, ulcerative painful bladder syndrome, and interstitial cystitis ${ }^{31,32}$, possibly suggesting a pathophysiological role for $\mathrm{BKPyV}$ in the development of these diseases.

$\mathrm{BKPyV}$ was associated with several types of cancer, including high-grade urothelial carcinoma (both in situ and invasive), micropapillary urothelial carcinoma, pleomorphic giant cell carcinoma, bladder adenocarcinoma, and nephrogenic adenoma ${ }^{3,5,6,7,8,9}$. Our study did not find any of these rarely seen types of urothelial cell carcinoma. Our cohort included conventional type urothelial cell carcinoma, and none of the samples were positive for BKPyV. Similar data were published previously. Csoma et al. reported no evidence of $\mathrm{BKPyV}$ in 76 bladder cancer samples in their cohort ${ }^{29}$. Kumari et al. reported no association of $\mathrm{BKPyV}$ with urothelial carcinoma in immunocompetent patients ${ }^{33}$. Lu et al. showed no association of $\mathrm{BKPyV}$ cytopathic effects in urine cytology with the high risk of developing a high-grade urothelial carcinoma ${ }^{24}$. In our study, the BKPyV in urine was not related to the urothelial cell carcinomas resected prior to the virus detection in urine, making its role in the oncogenesis of UCC very unlikely in our cohort.

Nevertheless, based on our results, BKPyV may be associated with intravesical therapy and/or radiotherapy since nine patients with UCC (9/14:64\%) in our cohort were treated with either BCG, mitomycin, or radiotherapy before the detection of the virus in urine. To the best of our knowledge, this is the first study reporting such findings. None of the patients had a decoy cell in the urine cytology prior to the diagnosis of UCC. Further research is necessary to clarify the exact mechanism of its occurrence or the nature of the association. It is known that intravesical treatment can lead to BCG cystitis and even disseminated systemic BCG infection ${ }^{34,35}$. Eosinophilic cystitis is a relatively common complication of intravesical mitomycin therapy ${ }^{36,37}$. Potentially, therapy-induced cystitis could explain the reactivation of the latent BKPyV. Still, this question was not the aim of our present study, and further research is needed to elucidate this issue. Moreover, the standard treatment of all patients diagnosed with UCC usually includes Bacillus 
Calmette-Guerin (BCG) or mitomycin C. Therefore, we cannot exclude that the other five patients with unknown UCC-treatment have also received the treatment, but we could not confirm this information in the database. However, it is known that reactivation of $\mathrm{BKPyV}$ can occur following various reasons, and further research is needed to elucidate its cause and mechanism in the case of intravesical treatment.

\section{CONCLUSIONS}

In our study, the BKPyV detected in urine was not associated with the prior resected urothelial cell carcinomas, potentially excluding the role of this virus in the oncogenesis of the conventional type UCC in our cohort. BKPyV-reactivation is not restricted to immunosuppression but also found in the urine of patients diagnosed with urothelial cell carcinoma and patients without any history of transplantation, malignancy, or chronic diseases. The intravesical treatment could be associated with the reactivation of the latent BKPyV. BKPyV-positivity in the immunocompetent patients may be explained by cystitis, possibly causing viral reactivation. BKPyV testing in post-UCC patients is of potential clinical relevance for the risk assessment of BKPyV-nephropathy, and further research is needed to unravel this complex relationship.

\section{Supplementary Materials:}

The following are available online at https://www.mdpi.com/1999-4915/12/12/1412/s1. Table S1: Clinicopathological data, demographic and relevant follow-up information of the patients without a history of urothelial cell carcinoma. Table S2: Clinicopathological, histopathological and immunohistochemistry data in the patients diagnosed with urothelial cell carcinoma (either in situ or invasive). Table S3: PCR-primers used in this study.

\section{Author Contributions}

Conceptualization, F.K., A.z.H., and I.V.S.; methodology, F.K., G.M., and I.V.S.; investigation, F.K., G.M., and I.V.S.; data curation, F.K., G.M., and I.V.S.; writingoriginal draft preparation, F.K., G.M., and I.V.S.; writing-review and editing, A.z.H., and I.V.S.; visualization, F.K., and I.V.S.; resources, I.V.S.; principal investigator, supervision, and project administration, I.V.S. and A.z.H. All authors have read and agreed to the published version of the manuscript.

\section{Funding}

This research received no external funding. 


\section{Conflicts of Interest}

The authors declare no conflict of interest. 


\section{References}

1. Roberts, I.S.; Besarani, D.; Mason, P.; Turner, G.; Friend, P.J.; Newton, R. Polyoma virus infection and urothelial carcinoma of the bladder following renal transplantation. Br. J. Cancer 2008, 99, $1383-1386$.

2. Bouvard, V.; Baan, R.A.; Grosse, Y.; Lauby-Secretan, B.; El Ghissassi, F.; Benbrahim-Tallaa, L.; Guha, N.; Straif, K. Carcinogenicity of malaria and of some polyomaviruses. Lancet Oncol. 2012, 13, 339-340.

3. Galed-Placed, I.; Valbuena-Ruvira, L. Decoy cells and malignant cells coexisting in the urine from a transplant recipient with BK virus nephropathy and bladder adenocarcinoma. Diagn. Cytopathol. 2011, 39, 933-937.

4. Zhang, Y.; Ahmed, H.; Haririan, A.; Ugarte, R.; Papadimitriou, J.C.; Drachenberg, C.B. Granulomatous inflammation in BK polyomavirus-associated nephropathy. Transpl. Infect. Dis. 2018, 20, e12939.

5. Liu, S.; Chaudhry, M.R.; Berrebi, A.A.; Papadimitriou, J.C.; Drachenberg, C.B.; Haririan, A.; Alexiev, B.A. Polyomavirus Replication and Smoking Are Independent Risk Factors for Bladder Cancer After Renal Transplantation. Transplantation 2017, 101, 1488-1494.

6. Papadimitriou, J.C.; Randhawa, P.; Rinaldo, C.H.; Drachenberg, C.B.; Alexiev, B.; Hirsch, H.H. BK Polyomavirus Infection and Renourinary Tumorigenesis. Am. J. Transplant. Off. J. Am. Soc. Transplant. Am. Soc. Transpl. Surg. 2016, 16, 398-406.

7. Alexiev, B.A.; Papadimitriou, J.C.; Drachenberg, C.B. BK polyomavirus-infected nephrogenic adenoma of the urinary bladder in a renal transplant recipient: A case report. Pathol. Res. Pract. 2015, 211, 697-701.

8. Alexiev, B.A.; Drachenberg, C.B.; Papadimitriou, J.C. Polyomavirus-cystitis associated with in situ and invasive urothelial carcinoma in a heart transplant recipient: Evidence suggesting sequential progression/evolution from infection to carcinoma. Transplantation 2015, 99, e3-e4.

9. Alexiev, B.A.; Papadimitriou, J.C.; Chai, T.C.; Ramos, E.; Staats, P.N.; Drachenberg, C.B. Polyomavirus (BK)-associated pleomorphic giant cell carcinoma of the urinary bladder: A case report. Pathol. Res. Pract. 2013, 209, 255-259.

10. Alexiev, B.A.; Randhawa, P.; Vazquez Martul, E.; Zeng, G.; Luo, C.; Ramos, E.; Drachenberg, C.B.; Papadimitriou, J.C. BK virus-associated urinary bladder carcinoma in transplant recipients: Report of 2 cases, review of the literature, and proposed pathogenetic model. Hum. Pathol. 2013, 44, 908-917.

11. Prado, J.C.M.; Monezi, T.A.; Amorim, A.T.; Lino, V.; Paladino, A.; Boccardo, E. Human polyomaviruses and cancer: An overview. Clinics 2018, 73 Suppl. S1, e558s.

12. Herawi, M.; Parwani, A.V.; Chan, T.; Ali, S.Z.; Epstein, J.I. Polyoma virus-associated cellular changes in the urine and bladder biopsy samples: A cytohistologic correlation. Am. J. Surg. Pathol. 2006, 30, 345-350.

13. Morace, R.; Kumar, T.; Tantisattamo, E.; Gibson, J.; Britton, S.; Li, W.; Kanaan, H.D.; Cohn, S.R.; Samarapungavan, D.; Zhang, P.L.; et al. Feasibility of BK Virus Real-Time PCR Testing in Renal Graft Biopsies With Negative SV40 Staining. Transplant. Proc. 2017, 49, 1294-1300. 
14. FEDERA. Human Tissue and Medical Research: Code of Conduct for Responsible Use; FEDERA: Nijmegen, The Netherlands, 2011.

15. Qamar, I.; Rehman, S.; Mehdi, G.; Maheshwari, V.; Ansari, H.A.; Chauhan, S. Utility of Cytospin and Cell block Technology in Evaluation of Body Fluids and Urine Samples: A Comparative Study. J. Cytol. 2018, 35, 79-82.

16. Klufah, F.; Mobaraki, G.; Chteinberg, E.; Alharbi, R.A.; Winnepenninckx, V.; Speel, E.J.M.; Rennspiess, D.; Olde Damink, S.W.; Neumann, U.P.; Kurz, A.K.; et al. High Prevalence of Human Polyomavirus 7 in Cholangiocarcinomas and Adjacent Peritumoral Hepatocytes: Preliminary Findings. Microorganisms 2020, 8, 1125.

17. Van Dongen, J.J.; Langerak, A.W.; Bruggemann, M.; Evans, P.A.; Hummel, M.; Lavender, F.L.; Delabesse, E.; Davi, F.; Schuuring, E.; Garcia-Sanz, R.; et al. Design and standardization of PCR primers and protocols for detection of clonal immunoglobulin and T-cell receptor gene recombinations in suspect lymphoproliferations: Report of the BIOMED-2 Concerted Action BMH4-CT98-3936. Leukemia 2003, 17, 2257-2317.

18. Yamada, Y.; Tsuchiya, T.; Inagaki, I.; Seishima, M.; Deguchi, T. Prediction of Early BK Virus Infection in Kidney Transplant Recipients by the Number of Cells With Intranuclear Inclusion Bodies (Decoy Cells). Transplant. Direct 2018, 4, e340.

19. Wu, Z.B.; Lin, G.B.; Zeng, A.P.; Chen, Z.Q.; Chen, J.; Zheng, M.Q.; Tu, G.W.; Rong, R.M. Detection of BK virus infection in renal transplant recipients and clinical application. Zhonghua Shi Yan He Lin Chuang Bing Du Xue Za Zhi = Chin. J. Exp. Clin. Virol. 2010, 24, 367-369.

20. Ranzi, A.D.; Prolla, J.C.; Keitel, E.; Brackmann, R.; Kist, R.; dos Santos, G.; Bica, C.G. The role of urine cytology for 'decoy cells' as a screening tool in renal transplant recipients. Acta Cytol. 2012, 56, 543-547.

21. Chakera, A.; Dyar, O.J.; Hughes, E.; Bennett, S.; Hughes, D.; Roberts, I.S. Detection of polyomavirus BK reactivation after renal transplantation using an intensive decoy cell surveillance program is costeffective. Transplantation 2011, 92, 1018-1023.

22. Geramizadeh, B.; Roozbeh, J.; Malek-Hosseini, S.A.; Azarpira, N.; Ayatollahi, M.; Salahi, H.; Aghdaee, M.; Yaghoobi, R. Urine cytology as a useful screening method for polyoma virus nephropathy in renal transplant patients: A single-center experience. Transplant. Proc. 2006, 38, 2923-2925.

23. Lee, K.W.; Lee, H.H.; Lee, D.S.; Kim, S.J.; Joh, J.W.; Oh, H.Y.; Kim, J.W.; Kim, Y.G.; Huh, W.S.; Kim, D.J.; et al. Polymerase chain reaction for the diagnosis of human polyomavirus-associated nephropathy in renal transplant recipients. Transplant. Proc. 2004, 36, 2116-2117.

24. Lu, H.; Elsheikh, T.M.; Zhang, Y. Polyomavirus (BK) cytopathic effect in urine cytology is not associated with high risk of developing high-grade urothelial carcinoma. J. Am. Soc. Cytopathol. 2020, 9, 84-88.

25. Costa, C.; Cavallo, R. Polyomavirus-associated nephropathy. World J. Transpl. 2012, 2, 84-94.

26. Mert, D.; Batgi, H.; Merdin, A.; Çeken, S.; Dal, M.S.; Tekgündüz, E.; Altuntaş, F.; Ertek, M. BK Virus-associated Hemorrhagic Cystitis in Patients with Allogeneic Hematopoietic Cell Transplantation: Report of Three Cases. Hematol. Rep. 2017, 9, 7205. 
27. Egli, A.; Infanti, L.; Dumoulin, A.; Buser, A.; Samaridis, J.; Stebler, C.; Gosert, R.; Hirsch, H.H. Prevalence of polyomavirus BK and JC infection and replication in 400 healthy blood donors. J. Infect. Dis. 2009, 199, 837-846.

28. Jamboti, J.S. BK virus nephropathy in renal transplant recipients. Nephrology 2016, 21, 647-654.

29. Csoma, E.; Bidiga, L.; Mehes, G.; Gergely, L. No Evidence of Human Polyomavirus 9, WU and KI DNA in Kidney and Urinary Bladder Tumour Tissue Samples. Pathobiol. J. Immunopathol. Mol. Cell. Biol. 2016, 83, 252-257.

30. Geetha, V.; Rao, L.; Monappa, V.; Susmitha, M.; Prabhu, R. Decoy cells in urine cytology: A useful clue to post-transplant polyoma virus infection. J. Cytol. 2012, 29, 133-134.

31. Reploeg, M.D.; Storch, G.A.; Clifford, D.B. BK Virus: A Clinical Review. Clin. Infect. Dis. 2001, 33, 191-202.

32. Winter, B.J.; O’Connell, H.E.; Bowden, S.; Carey, M.; Eisen, D.P. A Case Control Study Reveals That Polyomaviruria Is Significantly Associated with Interstitial Cystitis and Vesical Ulceration. PLoS ONE 2015, 10, e0137310.

33. Kumari, K.; Pradeep, I.; Kakkar, A.; Dinda, A.K.; Seth, A.; Nayak, B.; Singh, G. BK polyomavirus and urothelial carcinoma: Experience at a tertiary care centre in India with review of literature. Ann. Diagn. Pathol. 2019, 40, 77-80.

34. Yong, C.; Steinberg, R.L.; O’Donnell, M.A. Severe Infectious Complications of Intravesical Bacillus Calmette-Guérin: A Case Series of 10 Patients. Urology 2020, 137, 79-83.

35. Prasanna, T.; Craft, P.; Balasingam, G.; Haxhimolla, H.; Pranavan, G. Intravesical Gemcitabine versus Intravesical Bacillus Calmette-Guérin for the Treatment of Non-Muscle Invasive Bladder Cancer: An Evaluation of Efficacy and Toxicity. Front. Oncol. 2017, 7, 260.

36. Luckenbaugh, A.N.; Marks, R.M.; Miller, D.C.; Weizer, A.Z.; Stoffel, J.T.; Montgomery, J.S. A Management Algorithm for Mitomycin C Induced Cystitis. Bladder Cancer 2017, 3, 133-138.

37. Zhuo, C.; Li, X.; Zhuang, H.; Tian, S.; Cui, H.; Jiang, R.; Liu, C.; Tao, R.; Lin, X. Evaluating the efficacy and safety of intravesical chemotherapies for non-muscle invasive bladder cancer: A network meta-analysis. Oncotarget 2016, 7, 82567-82579. 


\section{Supplementary:}

Table S1. Clinicopathological data, demographic and relevant follow up information of the patients without a history of urothelial cell carcinoma.

\begin{tabular}{|c|c|c|c|c|c|c|}
\hline $\begin{array}{l}\text { Patient } \\
\text { Lab-ID }\end{array}$ & Age & Gender & $\begin{array}{l}\text { Follow } \\
\text { up period } \\
\text { (months) }\end{array}$ & $\begin{array}{l}\text { First presentation } \\
\text { with } B K P y V \text { urine } \\
\text { cytology }\end{array}$ & $\begin{array}{l}\text { BKPyV IHC } \\
\text { on cytology }\end{array}$ & $\begin{array}{l}\text { Patient history including transplantation } \\
\text { and immunosuppression }\end{array}$ \\
\hline I.1 & 25 & $\mathrm{M}$ & 12 & 2006 & + & Kidney transplantation \\
\hline I.2 & 57 & $\mathrm{~F}$ & 135 & 2007 & + & Kidney transplantation \\
\hline I.3 & 50 & $\mathrm{M}$ & 134 & 2007 & n.a. & Colitis ulcerosa \\
\hline I.4 & 60 & $\mathrm{M}$ & 7 & 2008 & + & Kidney transplantation \\
\hline I.5 & 74 & $\mathrm{M}$ & 12 & 2008 & + & Kidney transplantation \\
\hline I.6 & 63 & $\mathrm{M}$ & 85 & 2009 & + & $\begin{array}{l}\text { Autoimmune thyroiditis, diabetes, prostate } \\
\text { adenocarcinoma Grade group } 1\end{array}$ \\
\hline I.11 & 67 & $\mathrm{M}$ & 12 & 2011 & n.a. & No chronic disease \\
\hline I.14 & 72 & $\mathrm{M}$ & 75 & 2012 & + & Kidney transplantation 2011 \\
\hline I.16 & 55 & $\mathrm{~F}$ & 82 & 2012 & + & Autoimmune hepatitis \\
\hline I.18 & 66 & $\mathrm{M}$ & 27 & 2014 & + & $\begin{array}{l}2014 \text { colon adenocarcinoma. } 2015 \\
\text { High grade sarcoma soft tissue leg. No } \\
\text { information about treatment. }\end{array}$ \\
\hline I.19 & 78 & $\mathrm{M}$ & 12 & 2014 & + & No chronic disease \\
\hline I.20 & 38 & $\mathrm{~F}$ & 22 & 2014 & + & No chronic disease \\
\hline I.24 & 77 & $\mathrm{M}$ & 51 & 2015 & + & No chronic disease \\
\hline I.25 & 69 & $\mathrm{M}$ & 12 & 2016 & + & Stem cell transplantation, GVHD liver. \\
\hline I.27 & 76 & $\mathrm{M}$ & 16 & 2016 & n.a. & No chronic disease \\
\hline I.28 & 45 & $\mathrm{~F}$ & 48 & 2017 & + & No chronic disease \\
\hline
\end{tabular}

Abbreviations: M, Male; F, Female; BKPyV, BK polyomavirus; n.a. no material available for immunohistochemistry. 


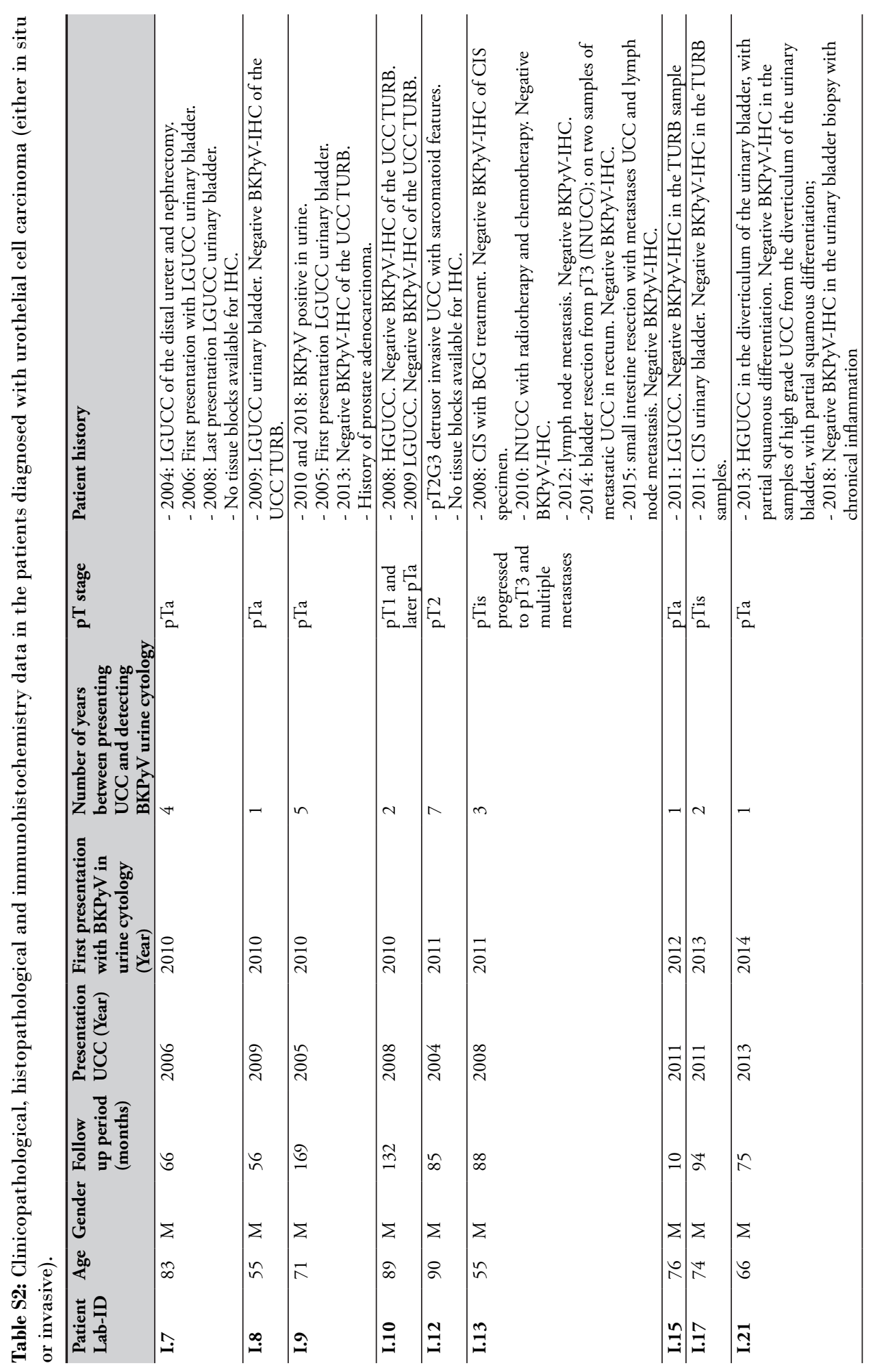



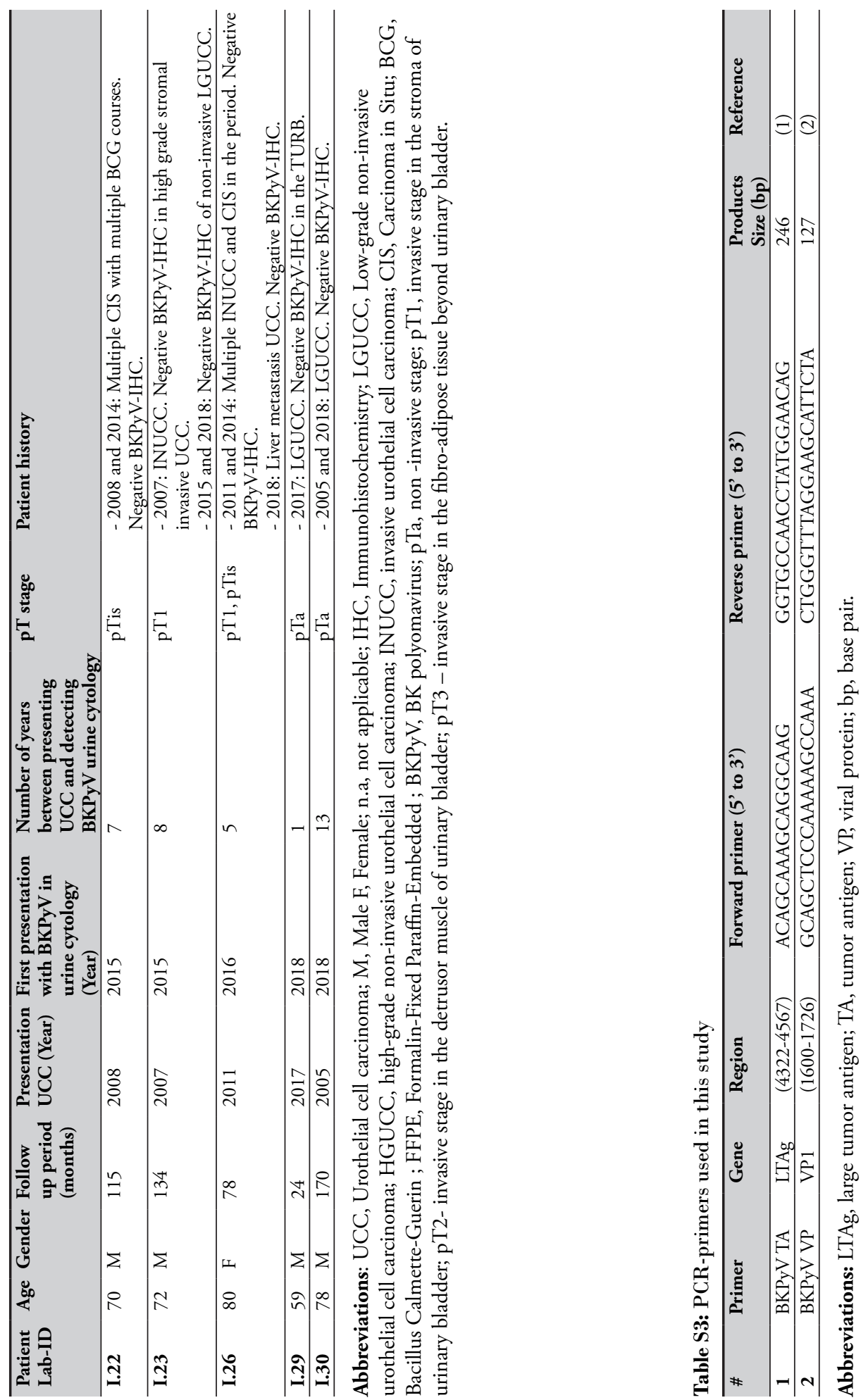


\section{References:}

1. Narayanan M, Szymanski J, Slavcheva E, Rao A, Kelly A, Jones K, et al. BK virus associated renal cell carcinoma: case presentation with optimized PCR and other diagnostic tests. Am J Transplant. 2007;7(6):1666-71.

2. Randhawa P, Kant J, Shapiro R, Tan H, Basu A, Luo C. Impact of genomic sequence variability on quantitative PCR assays for diagnosis of polyomavirus BK infection. J Clin Microbiol. 2011;49(12):4072-6. 
CHAPTER 5

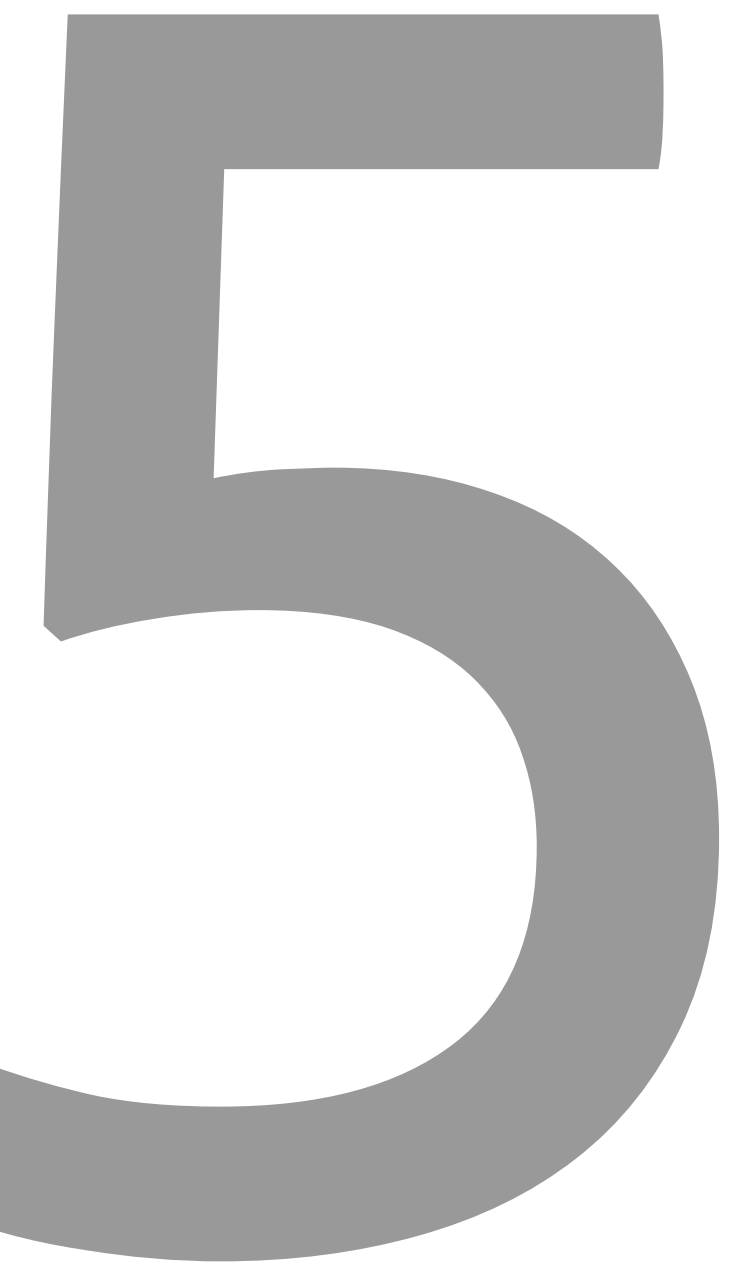




\section{Low Prevalence of Merkel Cell Polyomavirus in Human Epithelial Thymic Tumors}

Emil Chteinberg, Faisal Klufah, Dorit Rennspiess, Mick F. Mannheims, Myrurgia A. Abdul-Hamid, Mario Losen, Marlies Keijzers, Marc H. De Baets, Anna Kordelia Kurz, Axel zur Hausen 


\section{ABSTRACT}

\section{Background}

The etiology of thymic epithelial tumors is unknown. Murine polyomavirus strain PTA has been shown to induce thymomas in mice. Recently, using diverse molecular techniques, we reported the presence of human polyomavirus 7 (HPyV7) in thymic epithelial tumors. In the present study, we investigated the prevalence of Merkel cell polyomavirus $(\mathrm{MCPyV})$ in thymic epithelial tumors.

\section{Methods}

Thirty-six thymomas were screened for MCPyV by PCR and subsequently tested by DNA and RNA in situ hybridization and immunohistochemistry. Twenty-six thymomas were diagnosed with myasthenia gravis (MG).

\section{Results}

MCPyV DNA was detected by PCR in 7 (19.4\%) of the 36 thymic epithelial tumors and in six of these, the presence of $\mathrm{MCPyV}$ was confirmed by fluorescence situ hybridization. Of these, $3(28.6 \%)$ revealed weak MCPyV LT-antigen protein expression. In addition, one of the MCPyV positive thymomas tested positive for MCPyV LT RNA with RNAscope. Of interest, two out of the three thymomas that previously tested positive for MCPyV by immunohistochemistry also tested positive for HPyV7. One of the 11 MG-negative and 2 of the $25 \mathrm{MG}$-positive were positive for MCPyV.

\section{Conclusions}

$\mathrm{MCPyV}$ DNA and MCPyV protein expression can be detected in human epithelial thymoma; however, to a far lesser extent than HPyV7. Our data strongly indicate that because of its infrequent detection and weak expression, $\mathrm{MCPyV}$ is unlikely to play an important role in the etiopathogenesis of human thymomas.

Keywords: Fluorescence in situ hybridization; immunohistochemistry; Merkel cell polyomavirus; thymoma; tumorigenesis. 


\section{INTRODUCTION}

Thymomas are rare thymic epithelial tumors that are frequently associated with autoimmune diseases, such as myasthenia gravis (MG, 24-40\%).1 The underlying etiology of thymomas is unknown. Several studies have investigated the role of viruses in thymomagenesis. ${ }^{1-3}$ We previously assessed the presence of the novel human polyomaviruses 6 and 7 (HPyV6 and 7) in human thymic epithelial tumors, based on the reported induction of thymomas in mouse strains $\mathrm{C} 3 \mathrm{H} / \mathrm{BiDa}$ and $\mathrm{AKR}$ by the polyomavirus strain PTA, and frequently detected $\mathrm{HPyV7}$ in these tumors. ${ }^{4-6}$ The number of human polyomaviruses detected has continually increased in recent years. ${ }^{7,8}$ To date, only Merkel cell polyomavirus ( $\mathrm{MCPyV}$ ) has been established as a novel human tumor virus in the majority of Merkel cell carcinomas (MCC). ${ }^{8,9}$ In MCC, MCPyV is clonally integrated in the tumor DNA and harbors tumor specific truncating oncogenic mutations within the large T (LT) antigen. ${ }^{9-11}$ Truncated LT antigen and small T antigen (sTAg) of MCPyV have been shown to inhibit the tumor suppressor protein retinoblastoma (RB) and $\mathrm{p} 53 .{ }^{12-14} \mathrm{Nicol}$ et al. recently reported the seroprevalence of five novel human polyomaviruses (MCPyV, HPyV6, HPyV7, HPyV9, TSPyV [trichodysplasia spinulosaassociated polyomavirus]) is increased in an age-dependent manner. ${ }^{15}$ In other studies, $86.4 \%$ and $56.8-63.6 \%$ of adult blood sera ( $\geq 20$ years) were positive for MCPyV-and HPyV7-DNA, respectively. ${ }^{16,17}$ In two independent studies using clinicopathologically not further classified thymomas $(\mathrm{n}=10)$, no MCPyV DNA could be detected by PCR. ${ }^{18,19}$ Because of these reports and our recent finding that $62.2 \%$ of thymomas are HPyV7 positive, ${ }^{6}$ we aimed to comprehensively assess the prevalence of $\mathrm{MCPyV}$ in this clinicopathologically well-classified cohort of human thymic epithelial tumors by diverse molecular techniques.

Testing the prevalence of $\mathrm{MCPyV}$ in formalin-fixed and paraffin-embedded (FFPE) tissues of MCC has generated conflicting results in previous studies. ${ }^{20}$ According to Moshiri et al., the use of a PCR-based approach in combination with the CM2B4 anti LT-antigen antibody has the highest specificity and sensitivity. ${ }^{20}$ Herein, we used a PCR based approach in combination with MCPyV fluorescence situ hybridization (FISH) and immunohistochemistry (IHC) to elucidate the presence of $\mathrm{MCPyV}$ in thymic epithelial tumors.

\section{METHODS}

\section{Patients and tissues}

Thirty-six FFPE thymoma resection specimens were included in this study (from 19 women and 17 men; mean age 58.3 years, range 34-82 years). Thirty-five of these 
had previously been tested for the presence of HPyV7.6 Twenty-six thymoma patients were known to have a history of MG, of which 24 were anti-acetylcholine receptor (AChR) antibody positive and two were negative. Twelve of the $26 \mathrm{MG}$-positive thymoma patients were immunosuppressed. All clinical pathological data are summarized in Table 1. In addition, non-neoplastic thymic hyperplasia $(n=20)$ and fetal thymic $(n=20)$ tissues were included in this study (summarized in Table S1). All use of this tissue and patient data was in agreement with the Dutch Code of Conduct for Observational Research with Personal Data (2004) and Tissue (2011,www.federa.org/sites/default/files/ digital_version_first_part_code_of_conduct_in_uk_2011_12092012.pdf). Diagnoses were previously defined by histology in routine diagnostics and were reviewed by two experienced pathologists.

\section{Merkel cell polyomavirus (MCPyV) detection}

PCR was performed with 250 ng of genomic DNA using the AmpliTaq Gold DNA Polymerase (Thermo Fisher Scientific, Naarden, The Netherlands) in a final volume of 50 $\mu \mathrm{L}$, as recently described. ${ }^{10}$ DNA quality and integrity were assessed by specimen control size ladder (SCS) as previously described and published for the DNA used. ${ }^{6,21}$ All gained PCR fragments were confirmed by sequencing. In addition, Merkel cell carcinoma cell lines MS-1 and MCC26 were used as positive and negative controls, respectively, to detect MCPyV.

\section{MCPyV detection by fluorescence in situ hybridization (FISH)}

$\mathrm{MCPyV}$ FISH was performed as previously described. ${ }^{22,23}$ In brief, deparaffinized 3 $\mu \mathrm{m}$ thick sections were pretreated with $0.2 \mathrm{M}$ hydrochloric acid, incubated with $1 \mathrm{M}$ $\mathrm{NaSCN}$ and digested with $1 \mathrm{mg} / \mathrm{mL}$ pepsin (2500-3500 U/mg, Sigma Chemical, St. Louis, MO, USA). The biotin labeled "specific" MCPyV DNA probe was added to the samples at a concentration of $5 \mathrm{ng} / \mu \mathrm{L}$, followed by denaturation of DNA (five minutes, $\left.80^{\circ} \mathrm{C}\right)$ and hybridization overnight $\left(37^{\circ} \mathrm{C}\right.$, humid chamber; ThermoBrite System, Abbott Molecular, Abbot Park, IL, USA). Unbound MCPyV DNA probe was stringently washed away. Bound probe was detected by sequential incubation in a combination of secondary antibodies: fluorescein isothiocyanate (FITC) avidin secondary antibody (1:500) and biotin conjugated goat anti-avidin (1:100; Vector, Brunschwig Chemie, Amsterdam, The Netherlands). Prior to incubation, aspecific binding sites were blocked with Boehringer Blocking reagent (Roche, Molecular Diagnostics Inc., South Branchburg, NJ, USA). Cell nuclei were counterstained, and cover slipped with 4,6-diamidino-2-phenylindole dihydrochloride (DAPI; $0.2 \mu \mathrm{g} / \mathrm{mL}$; Vectashield, Vector Laboratories, Burlingame, CA, USA). Samples were visualized using a DM 5000B fluorescence microscope (Leica, Wetzlar, Germany) coupled to an online digital camera (Leica DC 300 Fx) for independent evaluation of FISH signals by two investigators. 


\section{RNA-in situ hybridization}

RNAscope 2.5 HD RED assay (cat. no. 322350; Advanced Cell Diagnostics, Newark, CA, USA) was performed using target probes to $\mathrm{MCPyV}$ on tissue sections according to the manufacturer's instructions and methods followed by Wang et al. ${ }^{24}$ Human peptidylprolyl isomerase B (Hs-PPIB) and bacterial dihydrodipicolinate reductase (DapB) were used as positive and negative controls, respectively. FFPE HeLa cells (Advanced Cell Diagnostics) were also used as negative and positive controls, as suggested by Advanced Cell Diagnostics. In addition, we used a FFPE MCC tissue block that previously tested $\mathrm{MCPyV}$-positive by DNA-PCR and immunohistochemistry as a positive control. Four investigators independently evaluated the slides.

\section{Immunohistochemistry (IHC) and double staining procedure}

We used an anti LT-antigen MCPyV antibody (clone: CM2B4, dilution 1:50; Santa Cruz Biotechnology Inc., Santa Cruz, CA, USA). Immunohistochemical staining was conducted on a Dako Autostainer Link 48 using the EnVision FLEX Visualization Kit K8008 (Dako, Carpinteria, CA, USA) according to standard diagnostic routine protocols and manufacturer's instructions.

\section{RESULTS}

\section{MCPyV detection by DNA PCR and FISH}

A total of 13 of 36 (36.1\%) thymomas tested positive using M1/2, 8 (22.2\%) tested positive using VP1, and 4 tested positives using LT3 primers (Table 1). Sequence analyses of the PCR amplicons identified all PCR products as MCPyV DNA sequences. In total, 7 (19.4\%) of 36 thymomas tested positive for two out of three different MCPyV DNA PCRs (Table 1, Fig 1) and thus were considered MCPyV-positive. Of these seven $\mathrm{MCPyV}$-positive thymomas, five had a history of MG. In these five, the presence of MCPyV was confirmed by MCPyV FISH (Fig 2a). FISH analysis revealed relative weak punctuate hybridization signals, which were mostly heterogeneous patterns in all cases tested. None of the follicular hyperplasia or fetal thymus tissues were positive for MCPyVDNA.

\section{MCPyV LT IHC and MCPyV LT RNA-ISH}

All seven positive MCPyV thymomas were assessed for MCPyV LT-antigen expression by IHC. Three out of seven (42.9\%) revealed the presence of LT-antigen in thymoma tissues (Fig 2e, Table 1). The expression of MCPyV LT in these tissues was weak. In addition, three thymoma tissues, two MCPyV-negative (ID 1-26 and ID 1-32) and one MCPyV-positive (ID 1-8); and one MCPyV-positive MCC tissue were analyzed for MCPyV LT-antigen RNA (Fig 2b,d, Table 1). The MCC cells revealed strong MCPyV 


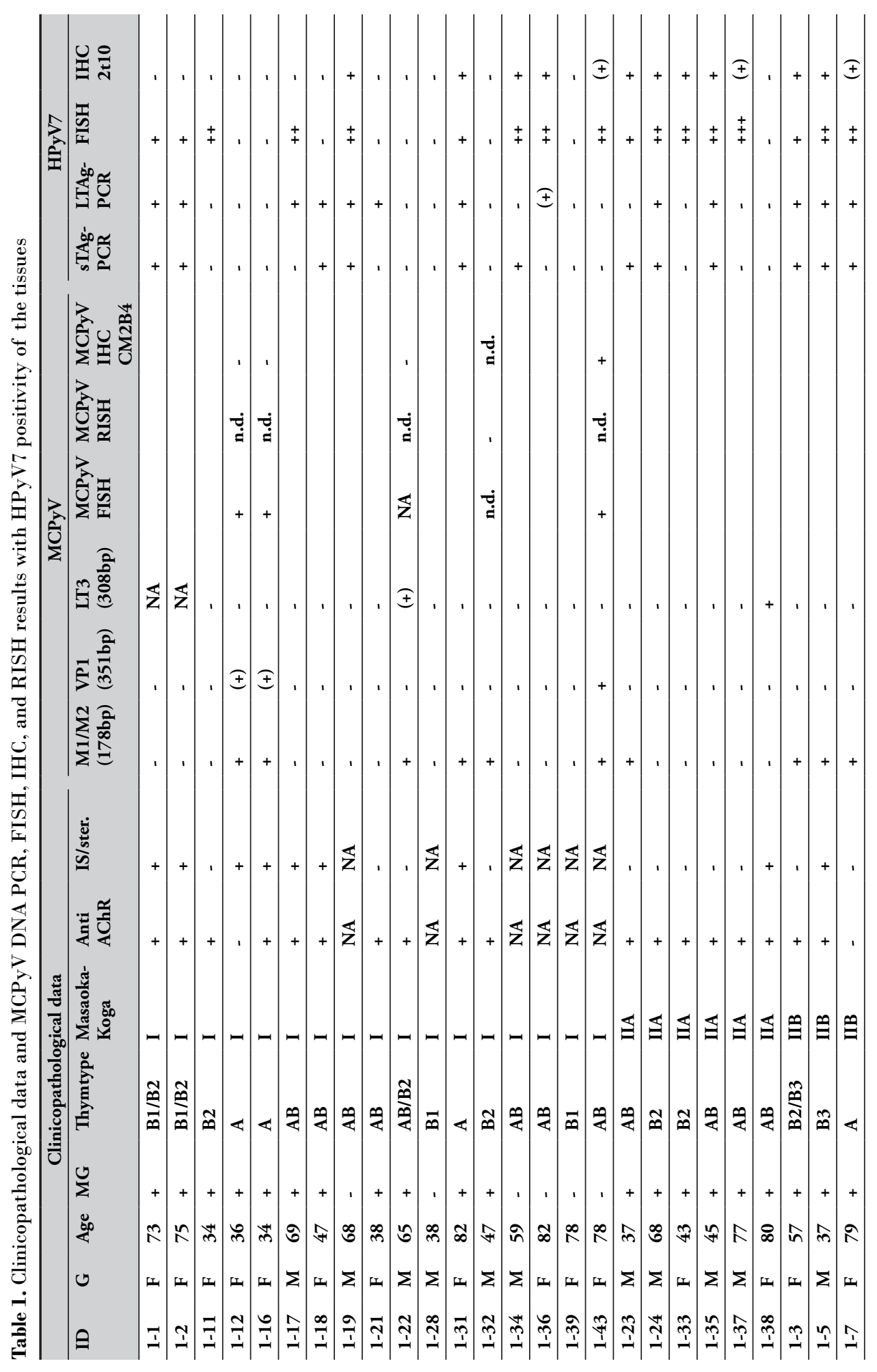




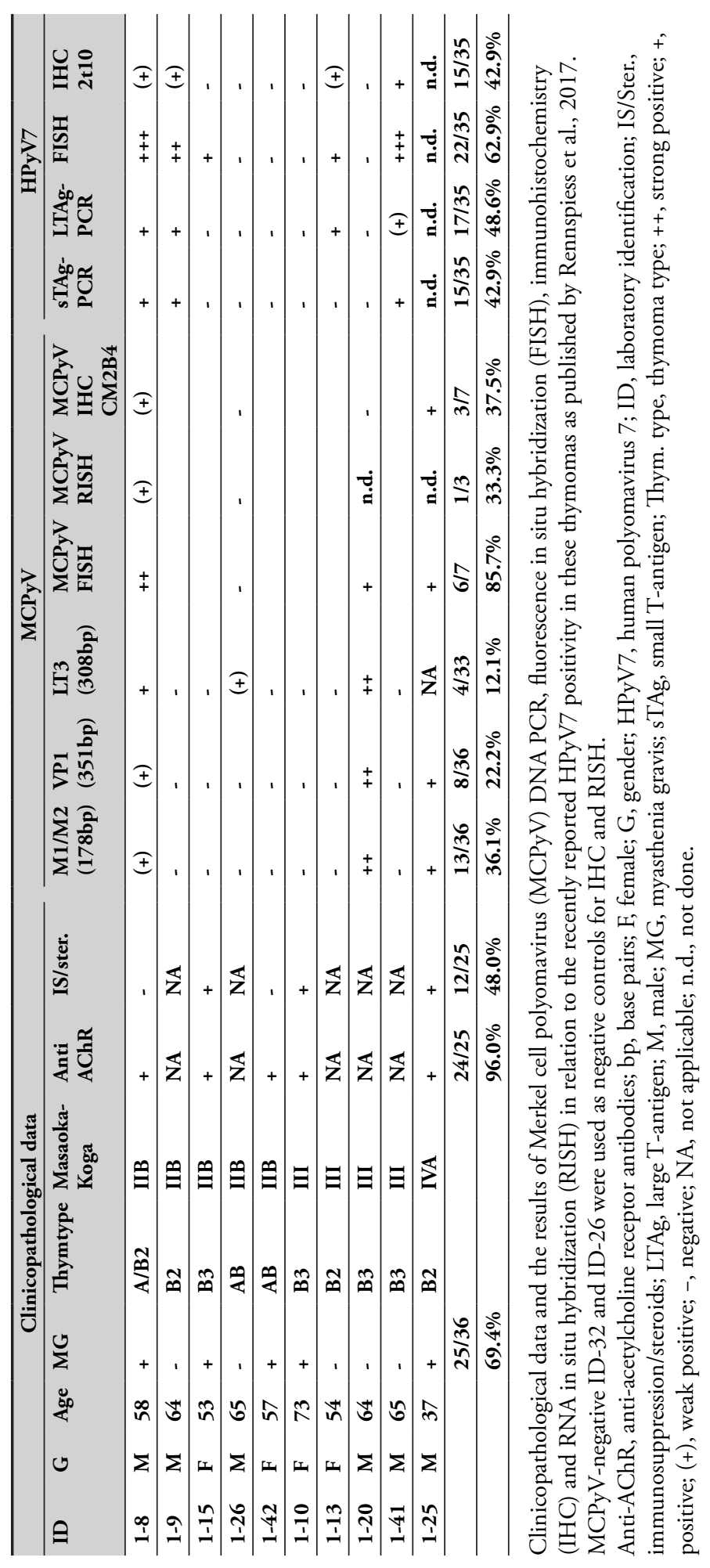




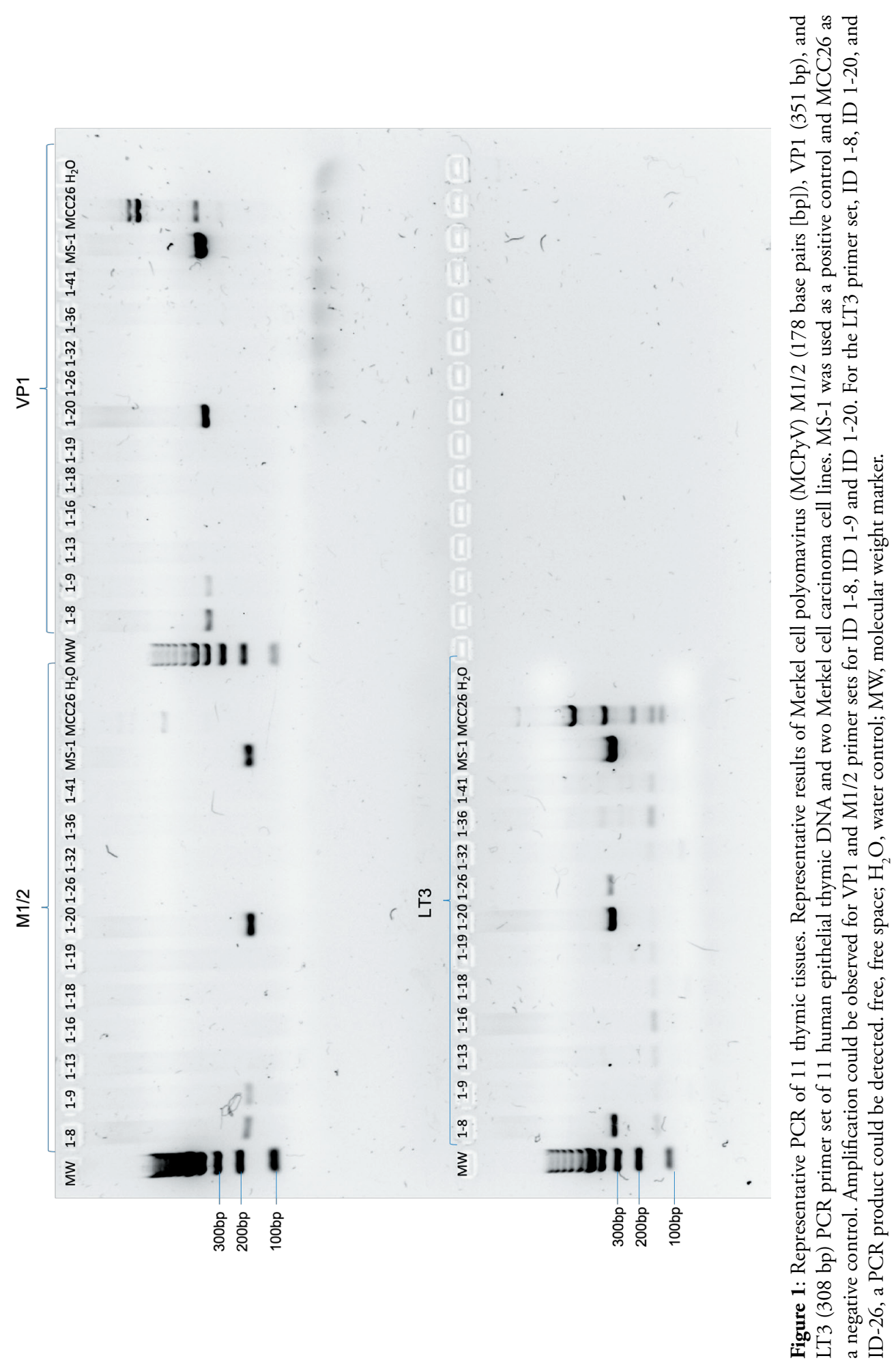


RNA detection in the cytoplasm (Fig 2c). ID 1-8 showed weak detection of MCPyV RNA. ID 1-26 and ID 1-32 showed no RNA amplification (Fig 2c, Table 1).

Of interest, thymoma tissue ID 1-8 was positive for both polyomaviruses: HPyV7 and $\mathrm{MCPyV}$ (Fig 2f, Table 1), whereas the MCPyV expression was weaker compared to HPyV7.

\section{DISCUSSION}

We recently reported the presence of $\mathrm{HPyV7}$ in $46.0 \%$ (protein level) to $62.2 \%$ (DNA level) of thymomas. 6 Herein, we assessed the presence of MCPyV on DNA level by PCR and FISH, on RNA level by RNA-ISH (RISH), and on protein level by IHC. In order to reliably detect MCPyV in FFPE tissues, Moshiri et al. recently reported that the use of a PCR-based approach in combination with the CM2B4 anti LT-antigen antibody yielded the highest specificity and sensitivity. ${ }^{20}$ In the present study, we used a PCR based approach in combination with MCPyV FISH, RISH, and IHC in order to comprehensively elucidate the presence of $\mathrm{MCPyV}$ in the same clinicopathologically well-classified thymoma cohort in which we recently assessed the presence of HPyV7. None of thymic hyperplasia $(\mathrm{n}=20)$ or fetal thymic tissues were positive for MCPyV assessed by PCR. Overall 19.4\% $(n=7)$ thymomas tested positive for the presence of MCPyV DNA (VP1, M1/2, and LT3). The presence of MCPyV DNA was confirmed in six of seven PCR positive thymomas by FISH analysis. The bands and signals obtained by PCR and FISH were weak. Correspondingly, weak MCPyV LT-antigen expression on protein level was found in three of seven thymomas. The prevalence of $\mathrm{MCPyV}$ was far lower than the prevalence of HPyV7 in these thymomas.

Of all MG-positive thymomas tested for MCPyV, five were positive on DNA level and three on protein level. Based on this small number, no conclusion can be drawn concerning an association between $\mathrm{MCPyV}$ and MG in thymoma. In addition, the weak DNA and protein detection correlates with the weak prevalence of MCPyV RNA compared to the RNA detection of the MCPyV-positive MCC tissue assessed by RISH in ID 1-8. In contrast to Torrachio et al. and Hashida et al. we used a larger number of $\mathrm{MCPyV}$ detection techniques in a larger thymoma cohort, which very likely explains why we were able to identify some $\mathrm{MCPyV}$ positive thymoma cases. ${ }^{18,19}$ However, given the weak DNA amplification and the low protein expression in these few, it can be concluded that $\mathrm{MCPyV}$ is weakly present in three thymomas, thus $\mathrm{MCPyV}$ is very unlikely to play a role in human thymomagenesis. In the context of our previously reported frequent finding of $\mathrm{HPyV} 7$ within the same thymoma cohort, the low prevalence of $\mathrm{MCPyV}$ indirectly supports a role of $\mathrm{HPyV} 7$ in human thymomagenesis. 

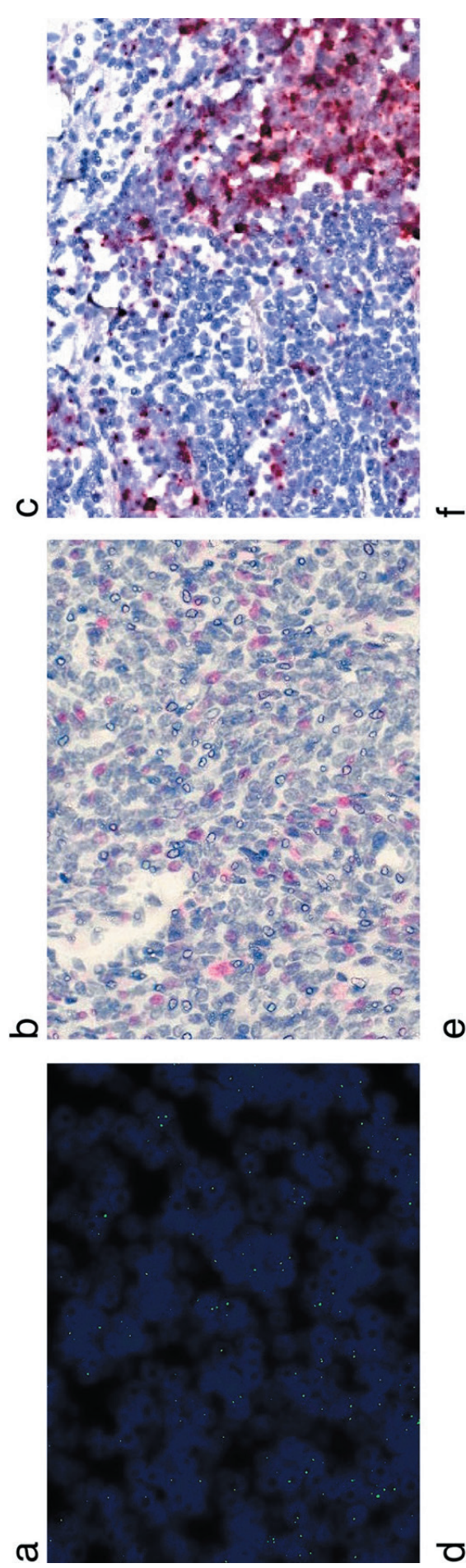

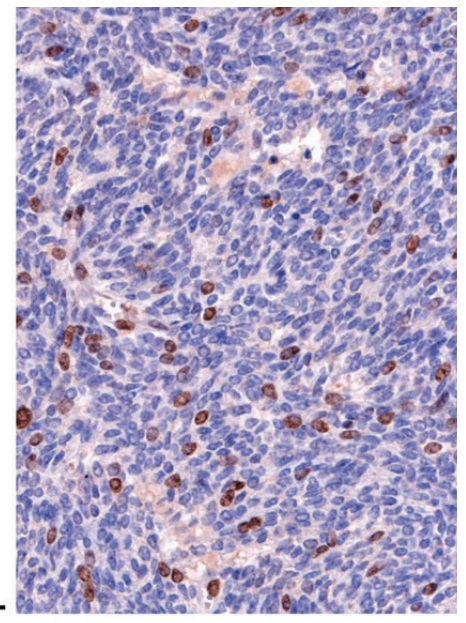

F⿻ำ 12. 9014 -

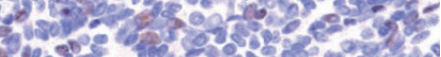

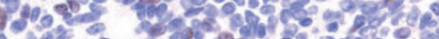

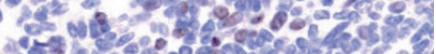

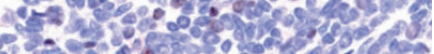

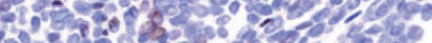

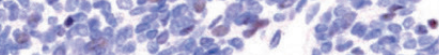
$0 \%$.

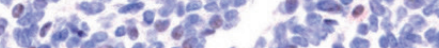

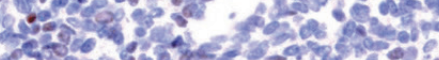

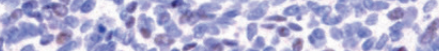

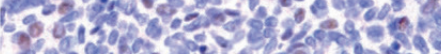

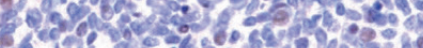

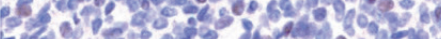

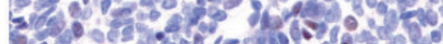
79.1.6.

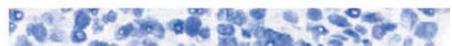
15270 - 13.5

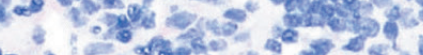
b s. itstogs

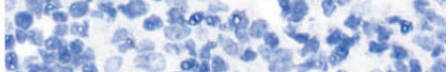
w - -20 s 3 a

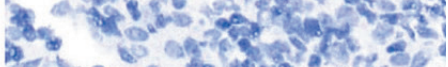

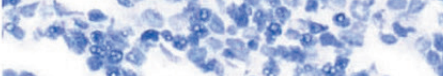

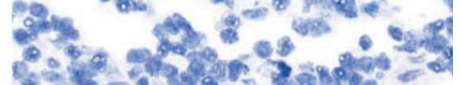

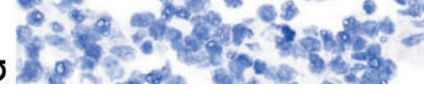

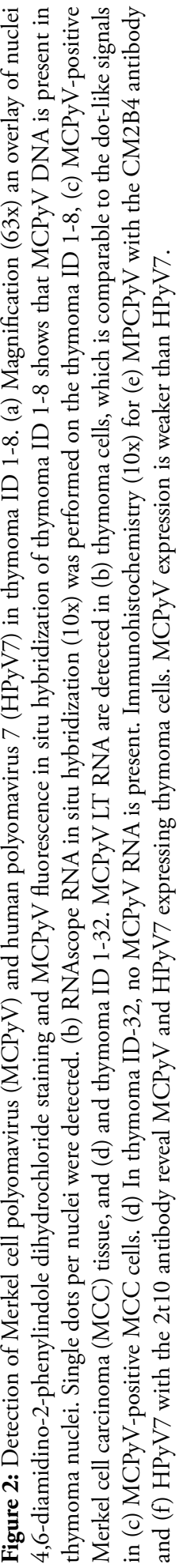


Remarkably, most thymoma cases that tested positive for $\mathrm{HPyV} 7$ or $\mathrm{MCPyV}$ in this cohort were of stage II or III according to the Masaoka classification (Table 1). Eleven of ${ }^{14}$ stage II thymomas were positive for one of these human polyomaviruses. In addition, one of the three MCPyV positive tissues was classified as stage IIIB (Table 1). The higher thymoma stages are considered to be more invasive. ${ }^{25,26} \mathrm{The}$ sTAg of MCPyV was recently observed to provide cells the ability to migrate; ${ }^{27}$ however, in as much this applies to HPyV7 and thymic epithelial cells currently remains speculation.

Of interest, to the best of our knowledge, we show for the first time the concomitant detection of $\mathrm{HPyV} 7$ and $\mathrm{MCPyV}$ in the same tumor tissue using diverse molecular techniques. Interestingly, $\mathrm{MCPyV}$ and $\mathrm{HPyV} 7$ are also present with a high seroprevalence in the same blood. ${ }^{16,17}$ Detection of the MCPyV genome by PCR and FISH reveals that $\mathrm{MCPyV}$ can infect thymic epithelial cells. In the present study we show that thymic epithelial tumors can harbor both $\mathrm{HPyV} 7$ and $\mathrm{MCPyV}$, although the prevalence of MCPyV in human epithelial tumors is far lower than reported for HPyV7. Our data strongly indicate that $\mathrm{MCPyV}$ is very unlikely to play an important role in the etiopathogenesis of human thymomas.

\section{Acknowledgments}

This research was supported by RWTH Aachen University through Graduiertenförderung nach Richtlinien zur Förderung des wissenschaftlichen Nachwuchses (RFwN).

\section{Disclosure}

No authors report any conflict of interest. 


\section{References:}

1. Cavalcante P, Barberis M, Cannone $\mathrm{M}$ et al. Detection of poliovirus-infected macrophages in thymus of patients with myasthenia gravis. Neurology 2010; 74: 1118- 26.

2. McGuire LJ, Huang DP, Teoh R, Arnold M, Wong K, Lee JC. Epstein-Barr virus genome in thymoma and thymic lymphoid hyperplasia. Am J Pathol 1988; 131: 385- 90.

3. Inghirami G, Chilosi M, Knowles DM. Western thymomas lack Epstein-Barr virus by Southern blotting analysis and by polymerase chain reaction. Am J Pathol 1990; 136: 1429- 36.

4. Sanjuan N, Porrás A, Otero J, Perazzo S. Expression of major capsid protein VP-1 in the absence of viral particles in Thymomas induced by murine polyomavirus. J Virol 2001; 75: 2891-9.

5. Wirth JJ, Fluck MM. Immunological elimination of infected cells as the candidate mechanism for tumor protection in polyomavirus-infected mice. J Virol 1991; 65: 6985- 8.

6. Rennspiess D, Pujari S, Keijzers M et al. Detection of Human Polyomavirus 7 in human thymic epithelial tumors. J Thorac Oncol 2015; 10: 360-6.

7. Gheit T, Dutta $S$, Oliver J et al. Isolation and characterization of a novel putative human polyomavirus. Virology 2017; 506: 45- 54.

8. Schadendorf D, Lebbé C, zur Hausen A et al. Merkel cell carcinoma: Epidemiology, prognosis, therapy and unmet medical needs. Eur J Cancer 2017; 71: 53-69.

9. Feng H, Shuda M, Chang Y, Moore PS. Clonal integration of a polyomavirus in human merkel cell carcinoma. Science (New York, NY) 2008; 319: 1096- 100.

10. Kassem A, Schöpflin A, Diaz C et al. Frequent detection of Merkel cell polyomavirus in human Merkel cell carcinomas and identification of a unique deletion in the VP1 gene. Cancer Res 2008; 68: 5009- 13 .

11. Shuda M, Feng H, Kwun HJ et al. T antigen mutations are a human tumor-specific signature for Merkel cell polyomavirus. Proc Natl Acad Sci 2008; 105: 16272- 7.

12. Houben R, Adam C, Baeurle A et al. An intact retinoblastoma protein-binding site in Merkel cell polyomavirus large $\mathrm{T}$ antigen is required for promoting growth of Merkel cell carcinoma cells. Int J Cancer 2012; 130: 847- 56.

13. Pipas JM, Levine AJ. Role of T antigen interactions with $\mathrm{p} 53$ in tumorigenesis. Semin Cancer Biol 2001; 11: 23- 30.

14. Harms PW, Patel RM, Verhaegen ME et al. Distinct gene expression profiles of viral- and non-viral associated Merkel cell carcinoma revealed by transcriptome analysis. J Invest Dermatol 2013; 133: 936- 45.

15. Nicol JTJ, Robinot R, Carpentier A et al. Age-specific seroprevalences of Merkel cell polyomavirus, human polyomaviruses 6,7 , and 9, and Trichodysplasia Spinulosa-associated polyomavirus. Clin Vaccine Immunol: CVI 2013; 20: 363- 8.

16. Šroller V, Hamšíková E, Ludvíková V, Musil J, Němečková Š, Saláková M. Seroprevalence rates of HPyV6, HPyV7, TSPyV, HPyV9, MWPyV and KIPyV polyomaviruses among the healthy blood donors. J Med Virol 2016; 88: 1254- 61. 
17. Kamminga S, van der Meijden E, Feltkamp MCW, Zaaijer HL. Seroprevalence of fourteen human polyomaviruses determined in blood donors. PLoS One 2018; 13: e0206273.

18. Toracchio S, Foyle A, Sroller V et al. Lymphotropism of Merkel cell polyomavirus infection, Nova Scotia, Canada. Emerg Infect Dis 2010; 16: 1702- 9.

19. Hashida $Y$, Imajoh M, Nemoto $Y$ et al. Detection of Merkel cell polyomavirus with a tumour-specific signature in non-small cell lung cancer. Br J Cancer 2013; 108: 629- 37.

20. Moshiri AS, Doumani R, Yelistratova L et al. Polyomavirus-negative Merkel cell carcinoma: A more aggressive subtype based on analysis of 282 cases using multimodal tumor virus detection. J Invest Dermatol 2017; 137: 819- 27.

21. van Dongen JJM, Langerak AW, Brüggemann $M$ et al. Design and standardization of PCR primers and protocols for detection of clonal immunoglobulin and T-cell receptor gene recombinations in suspect lymphoproliferations: Report of the BIOMED-2 Concerted Action BMH4-CT98-3936. Leukemia 2003; 17: 2257-317.

22. Haugg AM, Speel E-JM, Pantulu ND et al. Fluorescence in situ hybridization confirms the presence of Merkel cell polyomavirus in chronic lymphocytic leukemia cells. Blood 2011; 117: 5776- 7.

23. Haugg AM, Rennspiess D, Hausen AZ et al. Fluorescence in situ hybridization and qPCR to detect Merkel cell polyomavirus physical status and load in Merkel cell carcinomas. Int J Cancer 2014; 135: 2804- 15 .

24. Wang L, Harms PW, Palanisamy $\mathrm{N}$ et al. Age and gender associations of virus positivity in Merkel cell carcinoma characterized using a novel RNA in situ hybridization assay. Clin Cancer Res 2017; 23: $5622-30$.

25. Kolen KV, Pierrache L, Heyman S, Pauwels P, Schil PV. Prognostic factors and genetic markers in thymoma. Thoracic Cancer 2010; 1: 133- 40.

26. Huang J, Detterbeck FC, Wang Z, Loehrer PJ. Standard outcome measures for thymic malignancies. J Thorac Oncol 2010; 5: 2017- 23.

27. Knight LM, Stakaityte G, Wood JJ et al. Merkel cell polyomavirus small $\mathrm{T}$ antigen mediates microtubule destabilization to promote cell motility and migration. J Virol 2015; 89: 35- 47. 


\section{Supplementary data:}

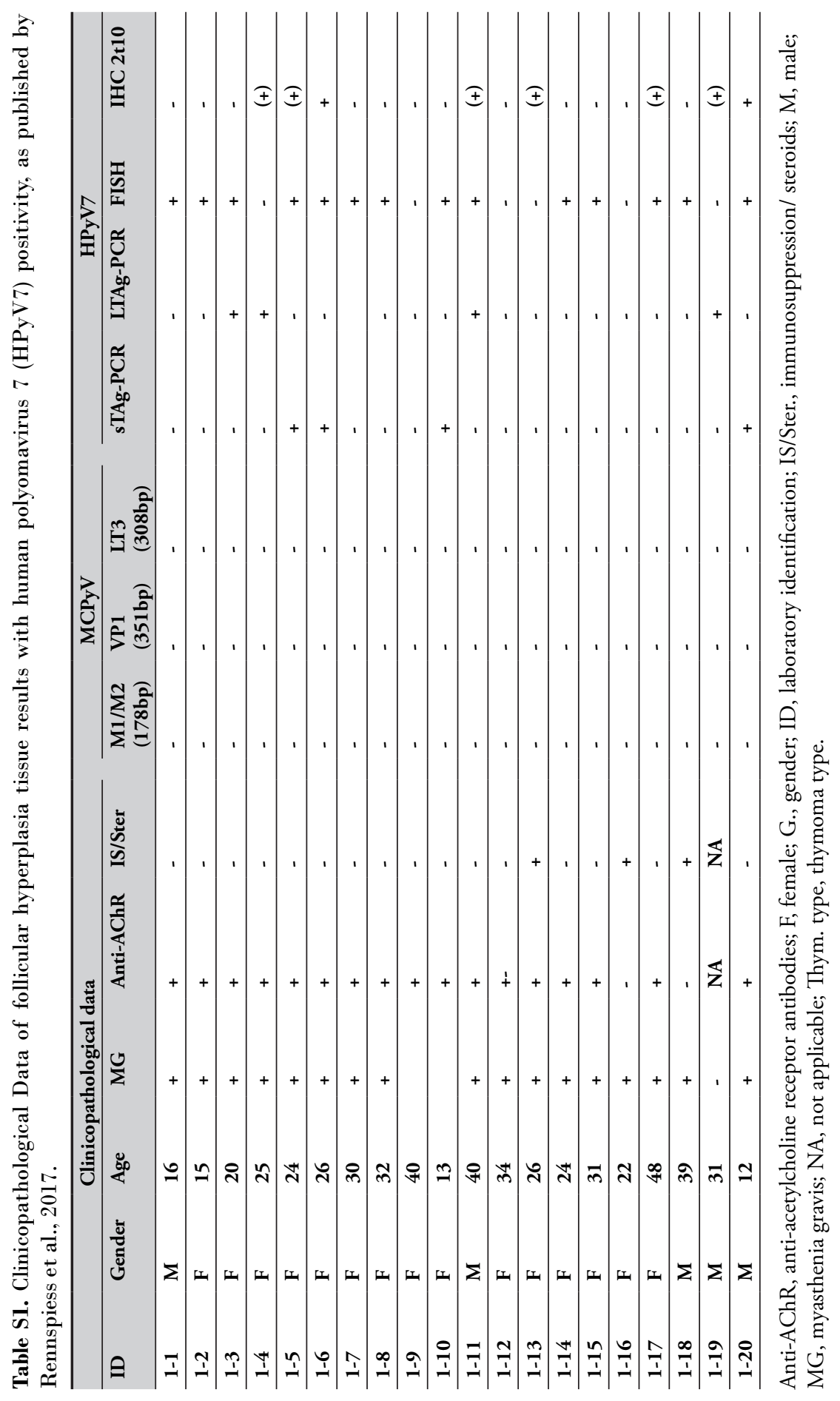




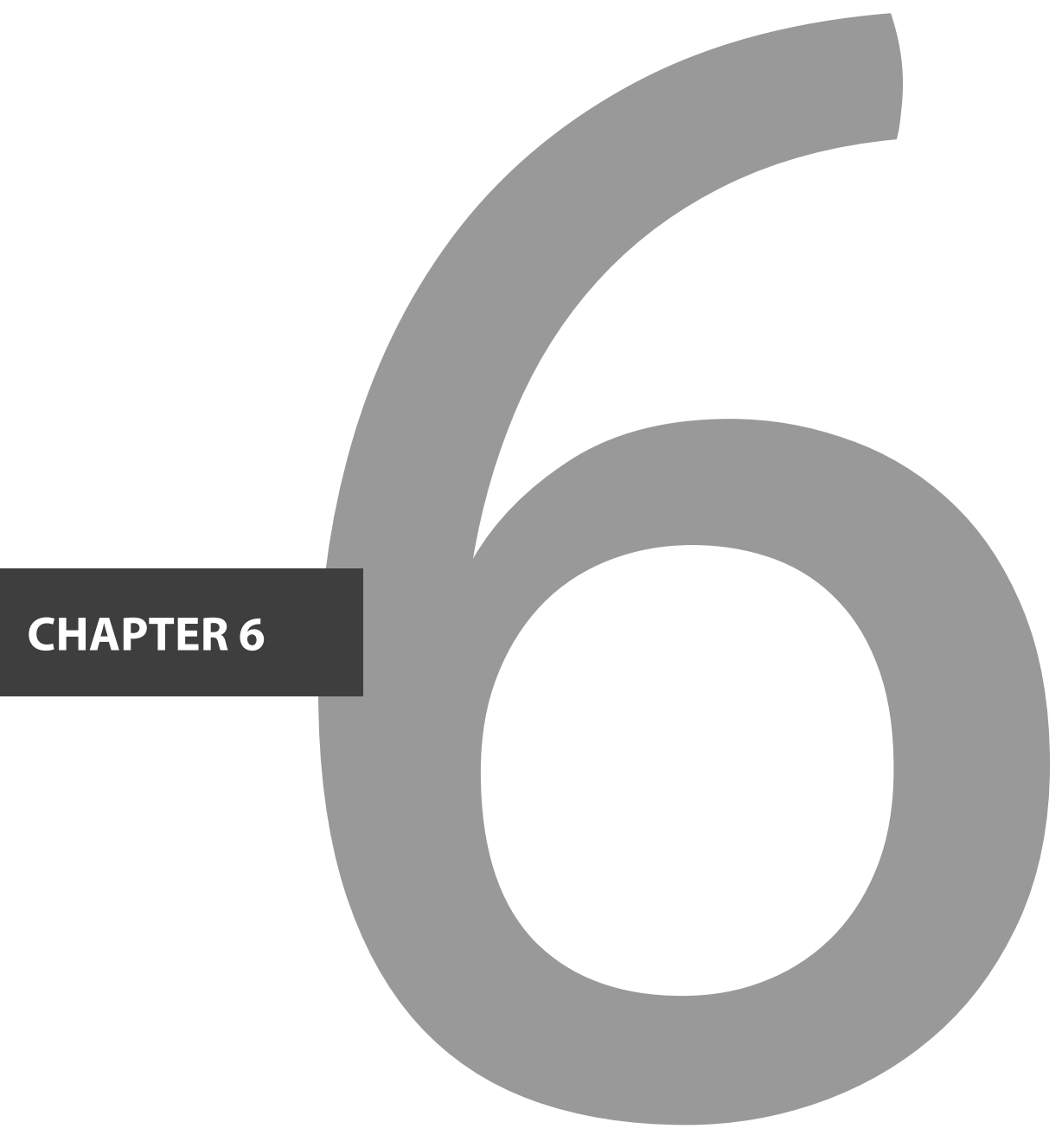




\section{Presence of Human Papillomavirus}

and Epstein-Barr Virus, but Absence of Merkel Cell Polyomavirus, in Head and Neck Cancer of Non-Smokers and NonDrinkers

Frans J. Mulder, Faisal Klufah, Famke M. E. Janssen, Farzaneh Farshadpour, Stefan M. Willems, Remco de Bree, Axel zur Hausen, Mari F. C. M. van den Hout, Bernd Kremer and Ernst-Jan M. Speel 


\section{ABSTRACT}

\section{Objective:}

Determine the presence and prognostic value of human papillomavirus (HPV), EpsteinBarr virus (EBV), Merkel cell polyomavirus $(\mathrm{MCPyV})$, and cell cycle proteins in head and neck squamous cell carcinoma (HNSCC) of non-smokers and non-drinkers (NSND).

\section{Methods:}

Clinical characteristics and tumors of 119 NSND with HNSCC were retrospectively collected and analyzed on tissue microarrays. RNAscope in situ hybridization (ISH) was used to screen for the presence of HPV and MCPyV mRNA. Immunohistochemistry was performed for expression of p16 as surrogate marker for HPV, Large T-antigen for $\mathrm{MCPyV}$, and cell cycle proteins $\mathrm{p} 53$ and $\mathrm{pRb}$. Positive virus results were confirmed with polymerase chain reaction. For EBV, EBV encoded RNA ISH was performed. Differences in 5-year survival between virus positive and negative tumors were determined by log rank analysis.

\section{Results:}

All oropharyngeal tumors (OPSCC) $(\mathrm{n}=10)$ were HPV-positive, in addition to one oral (OSCC) and one nasopharyngeal tumor (NPSCC). The other three NPSCC were EBV-positive. MCPyV was not detected. Patients with HPV or EBV positive tumors did not have a significantly better 5 -year disease free or overall survival. Over $70 \%$ of virus negative OSCC showed mutant-type p53 expression.

\section{Conclusion:}

In this cohort, all OPSCC and NPSCC showed HPV or EBV presence. Besides one OSCC, all other oral $(n=94)$, hypopharyngeal $(n=1)$, and laryngeal $(n=9)$ tumors were $\mathrm{HPV}, \mathrm{EBV}$, and $\mathrm{MCPyV}$ negative. This argues against a central role of these viruses in the ethiopathogenesis of tumors outside the oro- and nasopharynx in NSND. So, for the majority of NSND with virus negative OSCC, more research is needed to understand the carcinogenic mechanisms in order to consider targeted therapeutic options.

Keywords: head and neck cancer, human papillomavirus, Epstein-Barr virus, polyomavirus, non-smokers, nondrinkers, cell cycle protein, in situ hybridization 


\section{INTRODUCTION}

Viruses play an increasing role in head and neck squamous cell carcinoma (HNSCC). High-risk human papillomavirus (HPV)-positive oropharyngeal squamous cell carcinoma (OPSCC) has been identified as an entity with a different carcinogenesis than traditional HNSCC resulting from excessive tobacco and alcohol consumption. HPV is also an independent prognostic factor for a better disease free survival (DFS) and overall survival (OS), which has led to a down staging of these tumors in the eighth edition of the American Joint Committee on Cancer (AJCC) and union for International Cancer Control tumor-node-metastasis (TNM) classification ${ }^{1-3}$. Because of the better prognosis, de-escalation strategies are proposed for HPV-positive OPSCC patients ${ }^{4}$. The prevalence of HPV-positive OPSCC is rising in the Western World. A HPV prevalence above 50\% has already been reported in America, Europe, and Australia, based on HPV DNA in combination with either E6*I mRNA or p16 immunohistochemistry (IHC) detection ${ }^{5,6}$. Combining these HPV detection methods has been recommended because only OPSCC with transcriptionally active HPV is related to a better survival compared to biologically inactive infections ${ }^{7,8}$.

Another virus known for its carcinogenic potential in the head and neck region is the Epstein-Barr virus (EBV). EBV has a strong association with nasopharyngeal squamous cell carcinoma (NPSCC), approaching a prevalence of $100 \%$ in these tumors, and is endemic in Southern China, Southeast Asia, Northern Africa, and the Mediterranean basin ${ }^{9,10}$. It is suggested to cause an immunosuppressive microenvironment in these tumors, among others via PD-L1 overexpression, making these patients interesting candidates for checkpoint blockade therapy ${ }^{10}$. Detection of EBV presence can be performed reliably with EBV encoded RNA (EBER) in situ hybridization (ISH) ${ }^{9,11}$.

Lately, besides these acknowledged oncogenic viruses, there is attention for polyomaviruses in HNSCC. Merkel cell polyomavirus (MCPyV) has not only been detected by digital transcriptome subtraction and polymerase chain reaction (PCR) in up to $80 \%$ of Merkel cell carcinoma of the skin, but also in non-malignant tonsillar tissue, oral squamous cell carcinoma (OSCC), and pharyngeal cancer, with a reported prevalence of 23, 6.6-29, and $50 \%$ respectively ${ }^{12-17}$. Although it was thought not to play a role in oral carcinogenesis because of low viral loads detected with quantitative real-time PCR, the presence of $\mathrm{MCPyV}$ appears to be predictive for a better DFS ${ }^{16}$.

Cell cycle deregulation plays a central role in head and neck carcinogenesis, with frequent inactivation of TP53 and CDKN2A, leading to cell proliferation and prevention of apoptosis, among others. In HPV-related OPSCC, HPV integration in the host cell DNA genome leads to deregulation of oncoproteins E6 and E7, resulting in inactivity of 
$\mathrm{p} 53$ and retinoblastoma tumor suppressor gene product $\mathrm{pRb}$, respectively. The negative feedback of $\mathrm{pRb}$ inactivation leads to $\mathrm{p} 16$ overexpression ${ }^{18}$. In EBV infected NPSCC, it has been suggested that the cell cycle pathway is the most deregulated pathway, promoting the progression of the G1/S phase via inhibition of $\mathrm{p} 16$ expression and $\mathrm{pRb}$ overexpression ${ }^{19}$. For $\mathrm{MCPyV}$, oncogenetic transformation requires both integration of the viral genome into the host genome and truncation of the Large T-antigen (LTAg) to render the viral genome replication deficient ${ }^{20}$. LTAg mutations disrupt the DNA binding domain and the helicase domain distal to the pRb-binding motif, thereby promoting cell cycle progression by retaining its ability to bind to $\mathrm{pRb}^{20,21}$.

There is a small group of HNSCC patients without any exposure to the traditional risk factors. The mechanisms underlying carcinogenesis in these non-smokers and nondrinkers (NSND) remain largely unclear, but a significant role of oncogenic viruses would be expected. Indeed, a higher prevalence of HPV in these tumors has been reported in several studies, though in small numbers of patients ${ }^{22-25}$. Therefore, the goal of this study was to determine the presence of HPV, EBV, and MCPyV in a series of 119 wellcharacterized NSND with HNSCC. Secondary analyses evaluate differences in tumor suppressor proteins $\mathrm{p} 16, \mathrm{p} 53$, and $\mathrm{pRb}$ expression regarding viral presence and whether the presence of these viruses is predictive for a better DFS and OS.

\section{MATERIALS AND METHODS}

\section{Patients}

Consecutive patients with HNSCC were selected at the University Medical Center Utrecht (UMCU) and Maastricht University Medical Center (MUMC). In the UMCU, patients were prospectively selected between 1980 and 2004, as described previously ${ }^{26}$. In the MUMC, HNSCC patients have been selected retrospectively between 2011 and 2016, in addition to all patients with OPSCC between 2003 and 2010. Inclusion criteria were: $\geq 18$-years-old NSND patients with HNSCC, available formalin fixated and paraffin embedded (FFPE) tumor tissue, and $>2$ years follow up. Patient characteristics, risk factors, World Health Organization tumor classification, AJCC seventh edition staging, and information concerning recurrent disease or death were collected from the medical records. Non-smoking was defined as having no history of smoking, non-drinking as having no history of alcohol consumption (not even 'sporadic' alcohol consumption), as reported in the patients' medical records during both their first presentation at the Head and Neck outpatient clinic, as well as during the anesthesiological screening before panendoscopy or surgical resection. Patients with a second primary tumor in the head and neck region, tumors outside the upper aerodigestive tract, a cervical metastasis of 
unknown origin, or a histopathologic diagnosis other than squamous cell carcinoma were excluded.

The Medical Ethics Review Committee of the MUMC (2018-0567) has approved this study and the principles outlined in the Declaration of Helsinki were followed. All data and tissues were handled according to General Data Protection Regulation.

\section{Tissue Microarrays}

FFPE blocks of either the diagnostic biopsy or tumor resection were retrieved and hematoxylin and eosin sections were digitally evaluated with a senior head and neck pathologist (SW or MH), using Pannoramic viewer (3DHISTEC, Budapest, Hungary). Per patient, three $0.6 \mathrm{~mm}$ tumor tissue cores and one normal epithelium core were selected, placed in a tissue microarray (TMAs), and cut into $5 \mu \mathrm{m}$ sections.

\section{RNA In Situ Hybridization}

To screen for the presence of HPV and MCPyV mRNA, the RNAscope 2.5 RED assay kit and HPV-16/18 or V-MCPyV-LT-ST-Ag probe cocktails (Advanced Cell Diagnostics, Newark, California) were used according to the manufacturer's instructions. In short, TMA sections were deparaffinized and pretreated with RNAscope Hydrogen Peroxide for $10 \mathrm{~min}$. Antigen retrieval comprised of boiling the slide sections in the provided Target Retrieval Reagents solution at $100^{\circ} \mathrm{C}$ for $15 \mathrm{~min}$. After washing, the TMAs were dried over night at room temperature and treated for 30 min with RNAscope Protease Plus. In situ hybridization was performed applying four droplets of the provided probes prior to each of the six amplification steps $\left(30 \mathrm{~min}\right.$ at $40^{\circ} \mathrm{C}, 15 \mathrm{~min}$ at $40^{\circ} \mathrm{C}, 30 \mathrm{~min}$ at $40^{\circ} \mathrm{C}, 15 \mathrm{~min}$ at $40^{\circ} \mathrm{C}, 30 \mathrm{~min}$ at room temperature, and $15 \mathrm{~min}$ at room temperature, respectively). After each hybridization step, the slides were washed in the RNAscope wash buffer for $2 \mathrm{~min}$ at room temperature. Subsequent to alkaline phosphatase Fast Red chromogenic visualization of hybridized probes, the slides were counterstained with hematoxylin and assessed under a bright field microscope at 200x magnification. Tissue with at least 1 red punctate signal dot in the cytoplasm and/or nucleus of malignant cells was considered to be positive, as suggested by the manufacturer for genes with an expression level varying between 1 to $>10$ copies per cell. Probes for housekeeping gene transcript human peptidylprolyl isomerase B (Hs-PPIB) and bacterial dihydrodipicolinate reductase gene (dapB) transcript were used as positive and negative controls, respectively. As virus specific positive controls, virus positive tumor tissue was used, in addition to the HPV-18 positive cell line HeLa, HPV-16 positive cell lines SiHa and Caski, and MCPyV positive cell lines MKL-1, MKL-2, and WaGa (Figure 1). Negative controls were tumor tissue of a virus negative patient, cell lines MKL-1 and MKL-2 for HPV, and cell lines $\mathrm{MCC} 13$ and MCC26 for $\mathrm{MCPyV}^{27}$. 
EBER-ISH was performed using the Dako fluorescein-labeled EBV peptide nucleic acid (PNA) probe mixture and PNA ISH Detection kit (Agilent Technologies, Santa Clara, California) according to the manufacturer's instructions. Briefly, following TMA section deparaffinization, target retrieval was performed with the Dako Omnis ISH Pre-Treatment solution for $5 \mathrm{~min}$. Subsequently, enzyme pre-treatment was carried out using two steps of 3 min ethanol 96\% application and one step of Dako ISH Pepsin for $15 \mathrm{~min}$. Once the provided EBER RNA CISH probe was applied, denaturation at $66^{\circ} \mathrm{C}$ for $10 \mathrm{~min}$, and hybridization at $45^{\circ} \mathrm{C}$ for $90 \mathrm{~min}$ followed. The slides were washed with the ISH Stringent Wash Buffer for 3 min, and the provided reagents were applied for staining: CISH Endogenous Enzyme Block for $3 \mathrm{~min}$, Anti-FITc-AP for $30 \mathrm{~min}$, and BCIP-NBT Substrate for 15 min. Sections were counterstained with Nuclear Fast Red and analyzed under a bright field microscope at 200x magnification. Strong blue staining of more than $50 \%$ of tumor nuclei was considered to be positive. In parallel, a probe for housekeeping gene glyceraldehyde-3-phosphate dehydrogenase (GAPDH) was used to ensure the presence of mRNA in the TMA and a case of EBV-positive infectious mononucleosis served as a positive control (Figure 1).

\section{Immunohistochemistry}

Three- $\mu \mathrm{m}$ FFPE TMA sections were subjected to IHC, using primary monoclonal antibodies directed against p16, p53, pRb, and the LTAg of MCPyV (Table 1). Immunostainings were performed on a Dako Omnis autostainer (Agilent Technologies) using the EnVision FLEX+ Mouse (LINKER) kit. In short, antigen retrieval was performed on the TMA with sodium citrate-solution ( $\mathrm{pH}$ 6.0) for $\mathrm{p} 16$, or a high $\mathrm{pH}$ buffer ( $\mathrm{pH}$ 9.0) for $\mathrm{p} 53, \mathrm{pRb}$, and MCPyV. Endogenous peroxidase was blocked with Dako REAL Peroxidase-Blocking Solution prior to 20 minutes of incubation with the primary antibody. Binding of the antibodies was visualized by an enzymatic reaction with horseradish peroxidase and 3,3'-Diaminobenzidine as substrate, producing a brown precipitate (Figure 1). Slides were counterstained with hematoxylin and evaluated under a bright field microscope by two independent assessors, blinded for patients' clinical characteristics. In case of dissonance between the assessors, a third assessor evaluated the staining and agreement was reached by discussion. For p16, strong homogenous staining in the cytoplasm and nuclei of $>70 \%$ of the tumor cells was considered to be overexpression ${ }^{28}$. p53 staining was assessed as 0 -mutant type ( $0 \%$ nuclear staining), mutant-type overexpression ( $>70 \%$ strong nuclear staining in the non-keratinizing tumor cells), or wild-type (heterogeneous nuclear staining) ${ }^{29}$. For $\mathrm{pRb}$, nuclear staining in $<25 \%$ of tumors cells was evaluated as loss of $\mathrm{pRb}^{30}$. MCPyV LTAg was considered positive if $>10 \%$ of nuclei were stained ${ }^{31}$. For p16, a case of HPV-positive OPSCC was used as a control, and for $\mathrm{p} 53$ and $\mathrm{pRb}$ normal tonsil tissue was used. MCPyV positive cell lines MKL-1, MKL-2, and WaGa served as positive controls for the LTAg of MCPyV. 

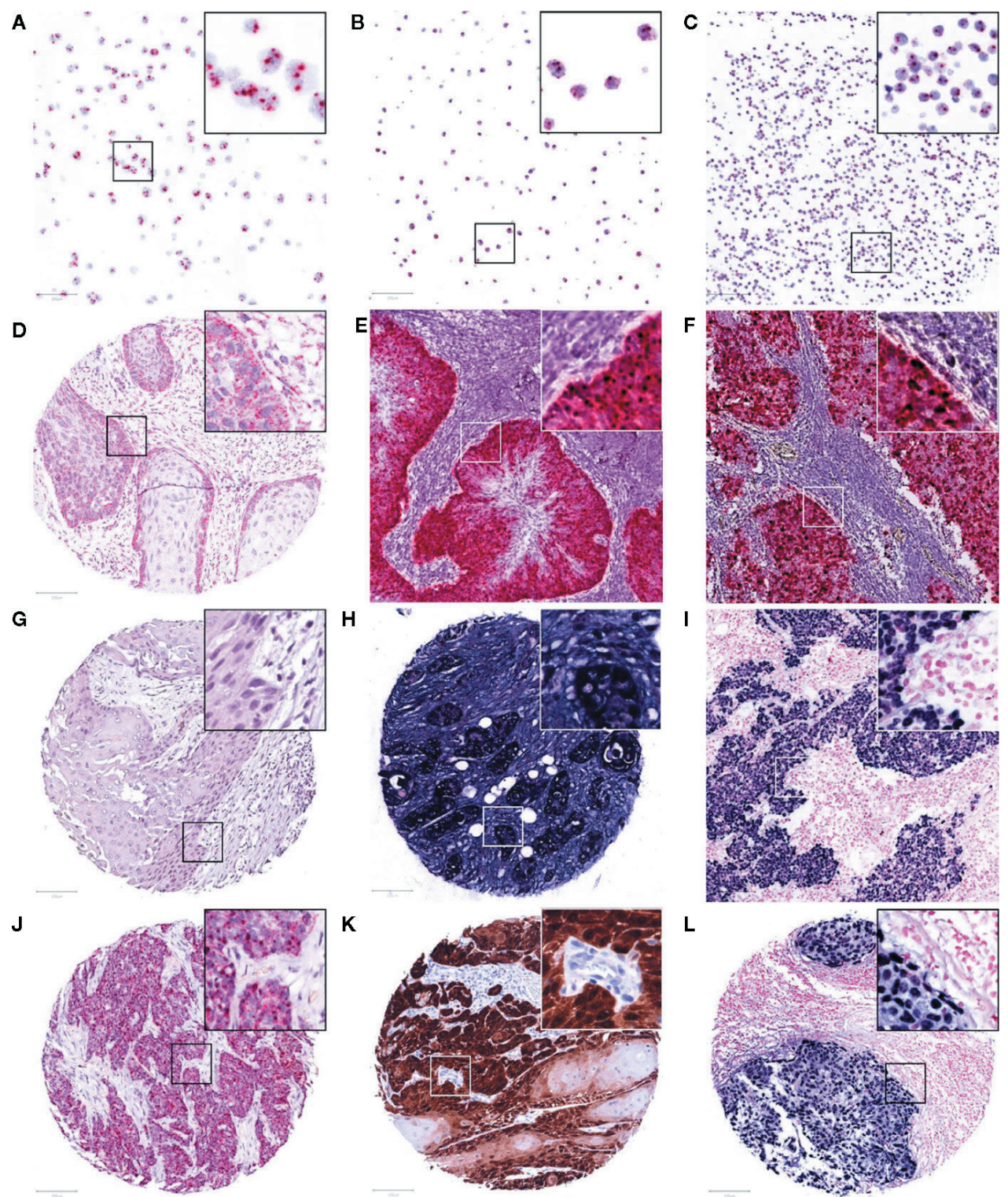

Figure 1 Representative images of RNAscope in situ hybridization on cell lines Caski (A), HeLa (B), and WaGa (C), positive for HPV-16, HPV-18, and MCPyV, respectively. A positive and negative control with housekeeping gene transcript PPIB (D) and bacterial transcript dapB (G) on the TMA, and a control patient positive for HPV-16 and MCPyV (E, F). Positive internal control for housekeeping gene GAPDH (H) and a control case of EBV-positive infectious mononucleosis (I) following Epstein-Barr virus encoded RNA in situ hybridization. Study TMA cores of patients positive for HPV-16 mRNA (at least 1 red punctate dot per tumor cell), p16 immunohistochemistry ( $>70 \%$ strong brown staining of tumor nuclei and cytoplasm), and EBV mRNA ( $>50 \%$ strong blue staining of tumor nuclei) are presented in $(\mathbf{J}-\mathbf{L})$, respectively. The images were taken at 200x magnification, an area of $100 \mu \mathrm{m} 2$ is marked in each image and 3x magnified in its top right corner. 
Table 1 Immunohistochemistry primary antibodies and evaluation criteria.

\begin{tabular}{|c|c|c|c|c|c|c|c|c|}
\hline \multicolumn{2}{|c|}{ Antibody characteristics } & \multirow[t]{2}{*}{ Source } & \multirow[t]{2}{*}{ Clone } & \multirow[t]{2}{*}{ Dilution } & \multirow[t]{2}{*}{ Retrieval } & \multirow[t]{2}{*}{ Localization } & \multicolumn{2}{|c|}{ Evaluation criteria } \\
\hline Antibody & Company & & & & & & Cut off & References \\
\hline p16 & Immunologic & $\begin{array}{l}\text { Monoclonal, } \\
\text { Mouse }\end{array}$ & MX007 & $1: 200$ & $\begin{array}{l}\text { Citrate } \\
(\mathrm{pH} 6.0)\end{array}$ & $\begin{array}{l}\text { Nuclear and } \\
\text { cytoplasmic }\end{array}$ & $>70 \%$ & 27 \\
\hline p53 & Dako Omnis & $\begin{array}{l}\text { Monoclonal, } \\
\text { Mouse }\end{array}$ & DO-7 & $\begin{array}{l}\text { Ready- } \\
\text { to-use }\end{array}$ & $\begin{array}{l}\text { High } \mathrm{pH} \text { buffer } \\
(\mathrm{pH} 9.0)\end{array}$ & Nuclear & $\geq 25 \%$ & 28 \\
\hline $\mathrm{pRb}$ & $\begin{array}{l}\text { Leica } \\
\text { Biosystems } \\
\end{array}$ & $\begin{array}{l}\text { Monoclonal, } \\
\text { Mouse }\end{array}$ & $13 \mathrm{a} 10$ & $1: 100$ & $\begin{array}{l}\text { High pH buffer } \\
(\mathrm{pH} 9.0)\end{array}$ & Nuclear & $\geq 25 \%$ & 29 \\
\hline $\begin{array}{l}\text { Large } \\
\text { T-antigen }\end{array}$ & $\begin{array}{l}\text { Santa Cruz } \\
\text { Biotechnology }\end{array}$ & $\begin{array}{l}\text { Monoclonal, } \\
\text { Mouse }\end{array}$ & CM2B4 & $1: 50$ & $\begin{array}{l}\text { High } \mathrm{pH} \text { buffer } \\
(\mathrm{pH} 9.0)\end{array}$ & Nuclear & $>10 \%$ & 30 \\
\hline
\end{tabular}

\section{Human Papillomavirus-Specific Polymerase Chain Reaction}

Of patients with a positive result for HPV RNA-ISH and/or p16 IHC, DNA was isolated from eight 5- $\mu \mathrm{m}$ FFPE whole tissue sections with the Maxwell RSC DNA FFPE kit (Promega, Madison, Wisconsin). DNA concentrations were determined using the Quantus Fluorometer and the QuantiFluor ONE dsDNA system (Promega). Next, 250 ng DNA was added to $1 \mathrm{ml}$ SurePath preservative fluid (VWR International, Amsterdam, Netherlands) and used for HPV-DNA analysis utilizing the COBAS 4800 platform (Roche, Basel, Switzerland), according to the manufacturer's instructions. The COBAS 4800 tests specifically for HPV-16, HPV-18, and a combination of 12 other HR-HPV

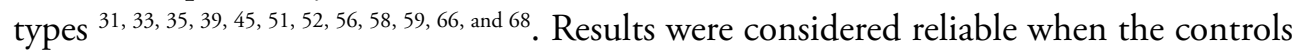
were labeled "valid". Housekeeping gene $\beta$-globin was used as a control for the human DNA, in addition to DNA samples of an HPV-positive and HPV-negative tumor.

\section{Statistical Analysis}

Patients were considered to be HPV or MCPyV positive when the virus was detected with at least two techniques (ISH, IHC and/or PCR). EBV presence was based on the EBER-ISH result. Differences in clinical parameters between virus positive and virus negative patients were evaluated by Mann-Whitney $U$ test for age because of a nonnormal distribution, Fisher's exact test for binominal variables (sex, M-stage, recurrence, p16, pRb), and the Fisher-Freeman-Halton exact test for tumor location, T-stage, N-stage, and $\mathrm{p} 53$ because of expected counts of less than five. The 5-year DFS and OS were estimated with Kaplan-Meier curves and differences between virus positive and negative tumors were determined by log rank test. DFS was defined as the last date of treatment until the biopsy date of a histologically proven recurrence or second primary tumor in the head and neck region. OS was defined as the time between the primary tumor biopsy date and death. Censoring took place when patients were lost to follow-up, deceased without recurrent disease for DFS, or at the cut-off point of 60 months. All clinical and pathological parameters were assessed in bivariate analysis regarding survival. Variables with significant $(\mathrm{p}<0.05)$ or near significant $(\mathrm{p}<0.1)$ relationships were evaluated in multinomial logistic regression to assess predictors for DFS and OS. Analyses were 
performed using IBM SPSS Statistics 25.0 (IMB corp., Armonk, NY) and a p-value of $<0.05$ was considered to be statistically significant.

\section{RESULTS}

A total of 119 patients were included in this study. These patients had a median age of 74.9 years (inter quartile range $=14.6$ years) and were mainly women $(78 \%)$ with a tumor of the oral cavity (80\%) and no regional or distant metastases (66 and 95\%, respectively). Thirty-one patients (26\%) had recurrent disease within 5 years (Table 2).

\section{Human Papillomavirus}

ISH on the TMAs showed HPV-16/18 mRNA expression in tumors of ten patients. All of these tumors showed p16 overexpression by IHC as well, in addition to five tumors with no HPV-16/18 mRNA expression. COBAS analysis on HR-HPV DNA in these 15 patients detected the presence of HPV-16 in ten tumors and another HR-HPV type in one other case. For patient 61, the quality of the DNA was insufficient for COBAS analysis. This resulted in a total of 12 tumors being HPV-positive based on at least two detection techniques (Table 3).

Compared to HPV-negative tumors, HPV-positive tumors were associated with lower age (67.3 versus 76.2 years old, $\mathrm{p}=0.003)$, oropharyngeal origin $(83 \%$ versus $0 \%, \mathrm{p}<$ 0.001 ), and $\mathrm{N} 2$-stage $(58 \%$ versus $13 \%, \mathrm{p}=0.004$ ) (Table 2 ). All oropharyngeal tumors $(\mathrm{n}=10)$ were HPV-positive, in addition to one OSCC of the alveolar process and a NPSCC. Although the OSCC case showed HPV-16 DNA and p16 overexpression, no mRNA was detected with ISH, neither on the TMA nor on a whole section.

Four of the twelve patients with HPV-positive tumors (33\%) died within five years, two of which had recurrent disease. For patient 1 there were no details recorded on the cause of death (OS = 59.6 months), patient 5 died of the complications of an aortic valve prosthesis endocarditis (OS $=23.7$ months), patient 21 received palliative treatment because of distant metastasis (DFS $=1.6$ months), and patient 61 developed liver metastases 20.1 months after initial therapy (Table 3 ). The presence of HPV was no predictor for a better DFS or OS ( $\mathrm{p}=0.33$ and $\mathrm{p}=0.27$, respectively), compared to HPV-negative HNSCC in NSND (Figures $2 \mathrm{~A}, \mathrm{~B}$ ). A younger age at cancer diagnosis ( $\mathrm{p}$ $=0.008)$, T1 stage ( $\mathrm{p}=0.0047)$, and N0 or N1 stage (both $\mathrm{p}<0.001)$ were retained in the best multivariable model as predictors for OS in HNSCC of NSND (Supplementary table 1, Table 4). The model explained $35 \%$ of the variation (model fit: omnibus test of model coefficients: X2 $=36.0$ and $\mathrm{p}<0.001$; $\mathrm{M}$-stage was omitted because of low case numbers). 


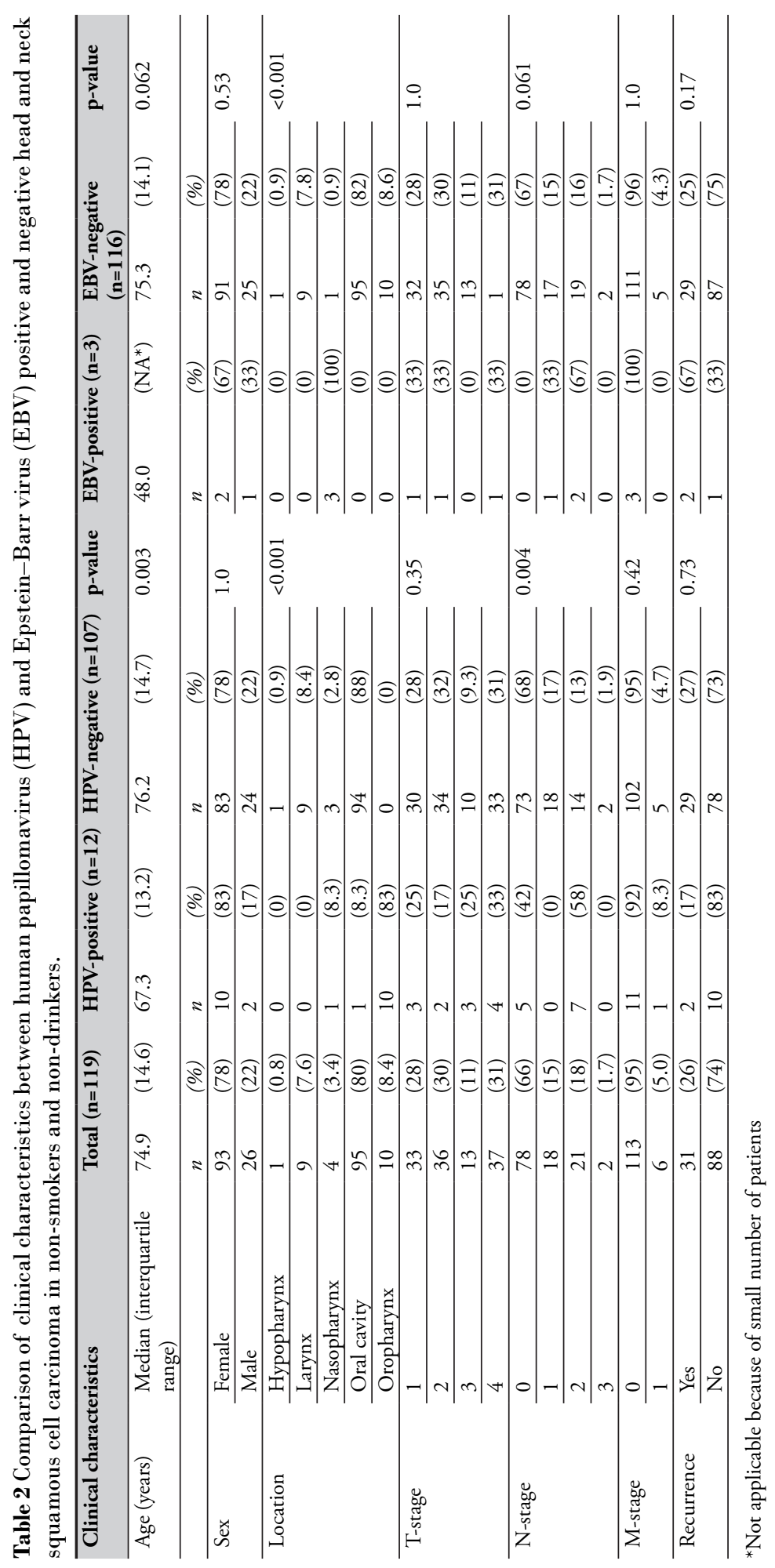


Table 3 Demographics and viral analysis results of 15 virus positive tumors in non-smokers and non-drinkers.

\begin{tabular}{|c|c|c|c|c|c|c|c|c|c|c|c|}
\hline Virus & $\begin{array}{l}\text { Study } \\
\text { ID }\end{array}$ & $\begin{array}{l}\text { Age } \\
\text { (years) }\end{array}$ & Sex & $\begin{array}{l}\text { Tumor } \\
\text { location }\end{array}$ & $\mathbf{T}$ & $\mathbf{N}$ & $\mathbf{M}$ & Recurrence & ISH & $\begin{array}{l}\text { p16 } \\
\text { IHC }\end{array}$ & $\begin{array}{l}\text { COBAS } \\
\text { PCR }\end{array}$ \\
\hline \multirow[t]{12}{*}{$\mathrm{HPV}$} & 1 & 56.4 & Male & Oropharynx & 3 & 2 & 0 & No & $++^{*}$ & + & HPV-16 \\
\hline & 3 & 53.9 & Female & Oropharynx & 1 & 2 & 0 & $\mathrm{No}$ & $+^{*}$ & + & HPV-16 \\
\hline & 4 & 71.5 & Male & Oropharynx & 4 & 2 & 0 & No & $+^{*}$ & + & HPV-16 \\
\hline & 5 & 76.6 & Female & Oropharynx & 2 & 2 & 0 & No & $+^{*}$ & + & HPV-16 \\
\hline & 9 & 69.5 & Female & Oropharynx & 3 & 0 & 0 & $\mathrm{No}$ & $-^{*}$ & + & HR-HPV \\
\hline & 16 & 68.3 & Female & Oropharynx & 1 & 0 & 0 & No & $+{ }^{*}$ & + & HPV-16 \\
\hline & 21 & 71.8 & Female & Oropharynx & 4 & 2 & 1 & Yes & $++^{*}$ & + & HPV-16 \\
\hline & 29 & 57.8 & Female & Nasopharynx & 2 & 0 & 0 & $\mathrm{No}$ & $++^{*}$ & + & HPV-16 \\
\hline & 32 & 63.5 & Female & Oral cavity & 4 & 0 & 0 & No & $-{ }_{-}^{*}$ & + & HPV-16 \\
\hline & 40 & 67.9 & Female & Oropharynx & 4 & 2 & 0 & No & $++^{*}$ & + & HPV-16 \\
\hline & 61 & 66.6 & Female & Oropharynx & 3 & 0 & 0 & Yes & $++^{*}$ & + & Invalid \\
\hline & 122 & 57.9 & Female & Oropharynx & 1 & 2 & 0 & No & $++^{*}$ & + & HPV-16 \\
\hline \multirow[t]{3}{*}{ EBV } & 6 & 48.0 & Male & Nasopharynx & 4 & 2 & 0 & Yes & $++^{\dagger}$ & NA & NA \\
\hline & 13 & 47.3 & Female & Nasopharynx & 2 & 1 & 0 & No & $++^{\dagger}$ & NA & NA \\
\hline & 18 & 74.6 & Female & Nasopharynx & 1 & 2 & 0 & Yes & $+^{\dagger}$ & NA & NA \\
\hline
\end{tabular}

ISH: in situ hybridization; IHC: immunohistochemistry; PCR: polymerase chain reaction; +: positive; -: negative; *: using RNAscope, ${ }^{\dagger}$ : using EBER-ISH, HR-HPV: high-risk human papilloma virus; EBV: Epstein-Barr virus; NA: not applicable

Table 4 Multivariable analysis of predictors for 5-year overall survival in non-smokers and non-drinkers with head and neck squamous cell carcinoma.

\begin{tabular}{|c|c|c|c|c|c|c|c|}
\hline \multirow[t]{2}{*}{ Parameter } & \multirow[t]{2}{*}{ Coefficient ( $\beta$ ) } & \multirow{2}{*}{$\begin{array}{l}\text { Standard } \\
\text { error }\end{array}$} & \multirow[t]{2}{*}{ Wald X ${ }^{2}$} & \multirow[t]{2}{*}{ OR } & \multicolumn{2}{|c|}{$95 \% \mathrm{CI}$} & \multirow[t]{2}{*}{ p-value } \\
\hline & & & & & Lower & Upper & \\
\hline Age & 0.053 & 0.020 & 7.0 & 1.05 & 1.0 & 1.1 & 0.008 \\
\hline \multicolumn{8}{|c|}{$N$-stage (reference T4) } \\
\hline T1 & -1.2 & 0.86 & 3.9 & 0.31 & 0.099 & 0.99 & 0.047 \\
\hline $\mathrm{T} 2$ & -0.67 & 0.54 & 1.5 & 0.51 & 0.18 & 1.5 & 0.22 \\
\hline T3 & 0.79 & 0.74 & 1.1 & 2.2 & 0.52 & 9.4 & 0.29 \\
\hline \multicolumn{8}{|c|}{$N$-stage (reference N3) } \\
\hline No & -19 & 0.59 & 1081 & $<0.001$ & $<0.001$ & $<0.001$ & $<0.001$ \\
\hline N1 & -17 & 0.78 & 506 & $<0.001$ & $<0.001$ & $<0.001$ & $<0.001$ \\
\hline N2 & -18 & 0.00 & NA & $<0.001$ & $<0.001$ & $<0.001$ & NA \\
\hline
\end{tabular}

NA: not available because of small number of cases

Nagelkerke $R^{2}=0.35$

HPV-positive tumors showed significantly more often p16 overexpression, p53 wild-type expression, and loss of $\mathrm{pRb}$ than HPV-negative tumors (p16: 100 versus 2.8\%, $\mathrm{p}<0.001$; p53: 83 versus 27\%, p < 0.001; pRb: 83 versus 19\%, p < 0.001) (Table 5).

\section{Epstein-Barr Virus}

Three tumors were EBV positive as detected by EBER-ISH. These patients all had a tumor of the nasopharynx (100 versus $0.9 \%$ in EBV-negative tumors, $\mathrm{p}<0.001$ ), resulting in virus positivity of all four nasopharyngeal tumors in this cohort (three containing EBV and one HPV). Two of the three patients (67\%) with EBV-positive tumors were below 50 years of age and non-Caucasian (Northern African and East Asian) and they all had regional metastases (Tables 2,3 ). 
A

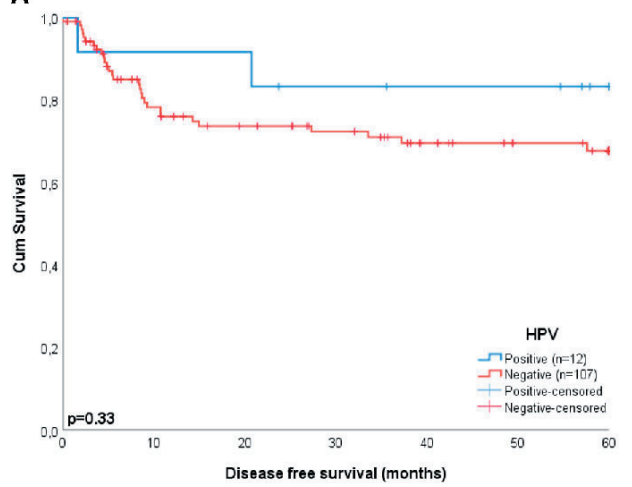

C

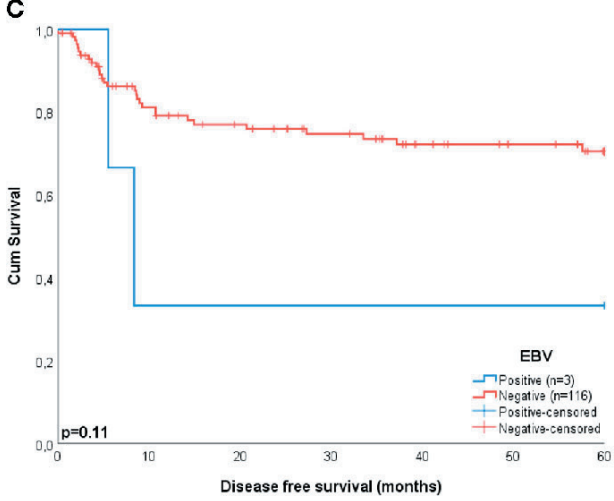

B

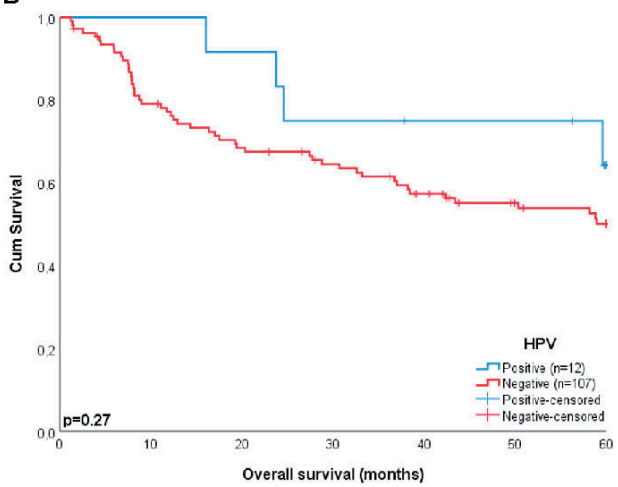

D

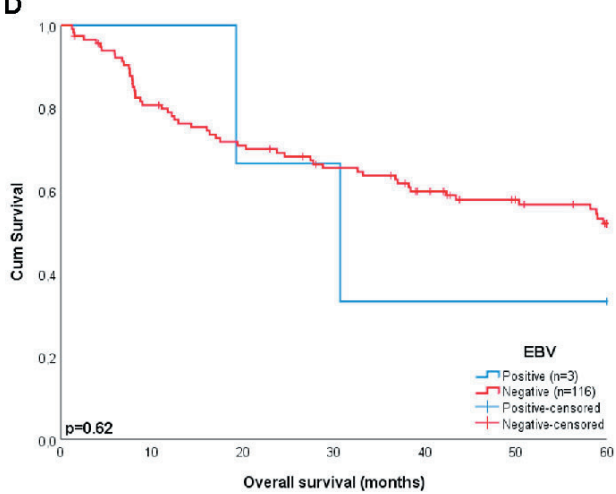

Figure 2 Kaplan-Meier curves estimating the survival of patients with HPV (A, B) and EBV (C, D) positive and negative tumors. HPV and EBV were no significant predictors for a better disease free $(p=0.33$ and $\mathrm{p}=0.11$, respectively) or overall survival $(\mathrm{p}=0.27$ and $\mathrm{p}=0.62$, respectively).

Patient 6 and 18 were both diagnosed with recurrent disease, the former with distant metastases 8.3 months after chemoradiotherapy and the latter with regional metastases 5.5 months after locoregional radiotherapy, which eventually led to their death. Although this resulted in a 33\% 5-year survival for patients with EBV-positive tumors, EBV was no significant predictor for DFS or OS in this cohort, compared to EBV-negative HNSCC $(\mathrm{p}=0.11$ and $\mathrm{p}=0.62$, respectively) (Figures $2 \mathrm{C}, \mathrm{D})$.

None of the EBV-positive tumors showed p16 overexpression, all were positive for $\mathrm{pRb}$ and two of the three tumors (66\%) showed p53 mutant-type overexpression. This did not differ significantly from cell cycle protein expression in EBV-negative tumors, most probably because of the small number of EBV-positive tumors (Table 5).

\section{Merkel Cell Polyomavirus}

$\mathrm{MCPyV}$ was not detected in any of the samples with RNA-ISH or IHC against the LTAg of MCPyV. 
Table 5 Expression of cell cycle proteins p16, p53, and $\mathrm{pRb}$ in virus positive and negative tumors.

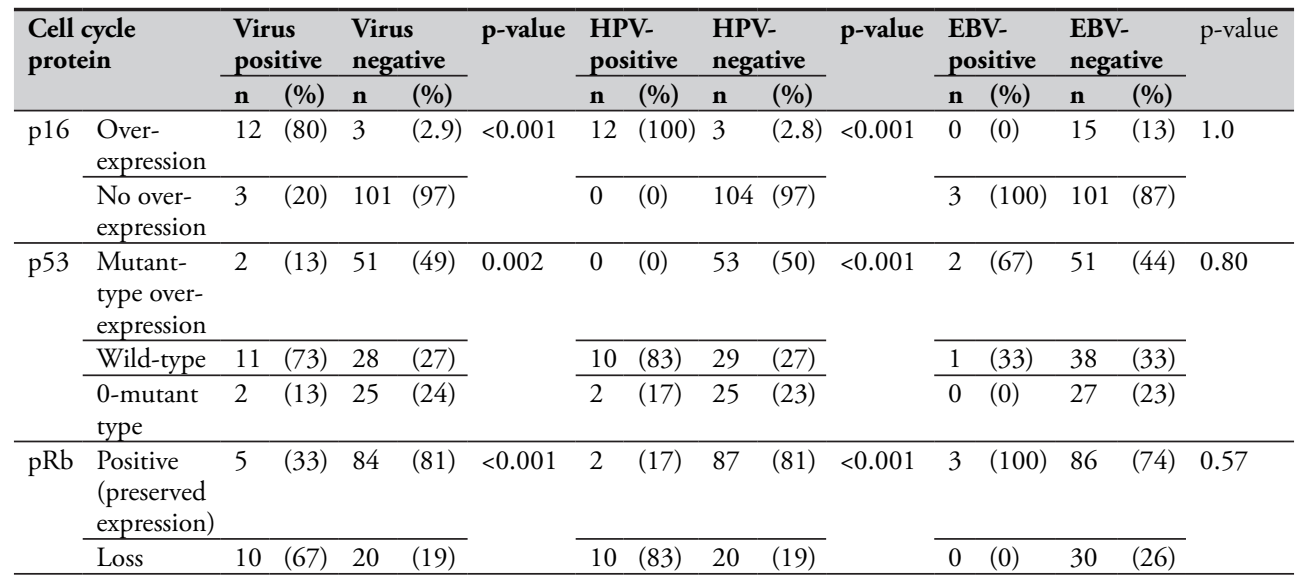

HPV, human papillomavirus; EBV, Epstein-Barr virus

\section{Virus Negative Tumors}

None of the squamous cell carcinomas of the oral tongue (OTSCC) ( $n=39$ ), larynx (LSCC) $(\mathrm{n}=9)$, or hypopharynx (HPSCC) $(\mathrm{n}=1)$ showed involvement of HPV, EBV, or MCPyV. Except for one tumor of the alveolar process, all other OSCC $(n=54)$ were virus negative as well (Table 2 ). Although the patients with virus negative tumors were relatively old (mean age of 75 years) when being diagnosed with HNSCC, the 5-year OS of these patients was still 50\% (Figure 2).

The three patients with virus negative OSCC containing p16 overexpression all had recurrent disease within 6 months after surgical resection [a T4N1M0 floor of mouth tumor, after an irradical resection the patient wished no further treatment (DFS $=0$ months), OS $=27.4$ months; a T4N0M0 oral cavity tumor (not otherwise specified), $\mathrm{DFS}=5.4$ months after resection, $\mathrm{OS}=8.2$ months; a T2N1M0 retromolar triangle tumor, DFS 4.8 months after resection, OS $=11.8$ months]. Two of these tumors showed loss of $\mathrm{pRb}$ expression. A poor DFS (2.3, 8.8, and 20.3 months) was also found in three other tumors in this cohort with some p16 expression (>50\%): all OTSCC with a pRb expression above $50 \%$. Apart from one LSCC, all tumors with loss of pRb were OSCC $(19 / 20)$ without any expression of $\mathrm{p} 16$. A younger age at cancer diagnosis $(\mathrm{p}=0.021)$ and N0 or N1 stage (both $\mathrm{p}<0.001$ ) were retained in the best multivariable model as predictors for OS in NSND with virus negative OSCC (Supplementary Table 2, Table 6). The model explained $31 \%$ of the variation (model fit: omnibus test of model coefficients: $\mathrm{X} 2=24.4$ and $\mathrm{p}<0.001 ; \mathrm{M}$-stage and $\mathrm{p} 16$ were omitted because of low case numbers).

Over $70 \%$ (76/104) of virus negative tumors showed mutant-type p53 expression, with 0 -type mutant expression in 20\% (19/94) of OSCC, 56\% (5/9) of LSCC, and 100\% 
Table 6 Multivariable analysis of predictors for 5-year overall survival in non-smokers and non-drinkers with virus negative oral squamous cell carcinoma.

\begin{tabular}{llllllll}
\hline Parameter & Coefficient $(\boldsymbol{\beta})$ & $\begin{array}{l}\text { Standard } \\
\text { error }\end{array}$ & Wald X & OR & & 95\% CI & p-value \\
\cline { 6 - 7 } & & & & Lower & Upper & \\
\hline Age & 0.051 & 0.022 & 5.3 & 1.05 & 1.0 & 1.1 & 0.021 \\
\hline N-stage (reference N3) & & & & & & \\
\hline N0 & -20 & 0.72 & 803 & $<0.001$ & $<0.001$ & $<0.001$ & $<0.001$ \\
\hline N1 & -18 & 0.89 & 414 & $<0.001$ & $<0.001$ & $<0.001$ & $<0.001$ \\
\hline N2 & -19 & 0.00 & NA & $<0.001$ & $<0.001$ & $<0.001$ & NA \\
\hline
\end{tabular}

NA: not available because of small number of cases

Nagelkerke $\mathrm{R}^{2}=0.31$

(1/1) of HPSCC, and mutant-type overexpression in 52\% (49/94) of OSCC and 22\% (2/9) of LSCC (Table 5). Cell cycle protein expression was no predictor for a better DFS or OS in patients with virus negative tumors (data not displayed).

\section{DISCUSSION}

The objective of this study was to determine if HPV, EBV, and MCPyV play a role in head and neck carcinogenesis of NSND, the role of cell cycle proteins p16, p53, and $\mathrm{pRb}$ regarding viral presence, and the influence of these viruses and proteins on patient survival. In this cohort of 119 NSND, the ten oropharyngeal (100\%) and four nasopharyngeal $(100 \%)$ tumors contained either HPV or EBV. Besides one oral cavity tumor, all other specimens of the oral cavity, hypopharynx, and larynx were HPV, EBV, and $\mathrm{MCPyV}$-negative. Virus positivity did not predict better disease free or overall survival. Regarding cell cycle protein expression, HPV-positive tumors showed more p16 overexpression, wild-type p53 expression, and loss of pRb compared to HPV-negative tumors. OSCC with $>70 \%$ p 16 expression had a poor DFS and OS, with loss of $\mathrm{pRb}$ in two of the three cases. The other $\mathrm{pRb}$ negative tumors were mainly OSCC as well and did not show p16 expression. Mutant type p 53 expression was observed in over $70 \%$ of virus negative HNSCC.

As the worldwide HPV prevalence is rising, a wide range has been reported in the literature, ranging from $20 \%$ in OPSCC patients from Eastern Asia or Central America, to over $50 \%$ in Europe and Australia, based on HPV DNA combined with E6*I mRNA or p16 IHC ${ }^{5,6}$. A recent systematic review specifically analyzing patients without tobacco or alcohol consumption reported an OPSCC HPV prevalence of over $60 \%$ in nonsmokers and over $40 \%$ in non-drinkers, compared to $20 \%$ in smokers and drinkers, based on at least two detection techniques (combining PCR, ISH, IHC, or sequencing) ${ }^{25}$. This is a lower prevalence than the 100\% (10/10) HPV infections of OPSCC in the current study. Possibly, the low number of OPSCC in the current study explains the $100 \%$ 
prevalence, as it could be a coincidence that they were all HPV-positive. Nonetheless, it is acknowledged that HPV plays an increasingly substantial role in OPSCC carcinogenesis of patients without the traditional risk factors. The HPV prevalence of $1.8 \%(2 / 109)$ in non-OPSCC as found in the current study is comparable to the low prevalence in other studies ${ }^{32-35}$.

In this study, 4.2\% (4/95) of OSCC showed p16 overexpression with IHC, and one of those contained HPV-16 DNA following COBAS analysis resulting in HPV-positivity according to detection by two methods. p16 overexpression could result from loss of pRb function via structural alterations, or maybe as a result of other oncogenic viruses affecting $\mathrm{pRb}$ expression that we are not aware of ${ }^{36}$. Lechner and colleagues speculated that high levels of protein p16 could also occur in cells irrespective of pRb expression or HPVpositivity, as a result of enrichment for NSD1 mutations in CDKN2A wild-type tumors 36, 37 . NSD1 is coding for Histone H3K36 methyltransferase, which is associated with DNA hypomethylation, resulting in 16 overexpression when mutated by not being able to regulate its expression via methylation anymore ${ }^{36}$. Indeed, $\mathrm{p} 16$ overexpression has been reported in non-OPSCC, without a correlation to HPV infection nor as a predictor for survival ${ }^{34,38}$. Therefore, The College of American Pathologists does not endorse routine p16 screening for non-OPSCC ${ }^{39}$. As the one HPV-positive OSCC in this cohort had no loss of $\mathrm{pRb}$ and lacked HPV mRNA in a whole section following RNAscope ISH analysis, the p16 overexpression could be a result of CDKN2A mutation, and HPV a commensal with the HPV DNA not located in the tumor cells but in the adjacent mucosal epithelial cells ${ }^{40}$. The three HPV-negative OSCC with p16 overexpression in the current study had a poor DFS. This could be a result of pRb loss, although for the whole study group p16 overexpression was no significant predictor for survival. Additionally, HNSCC could be the result of a genetic predisposition. OSCC has been associated with specific CDKN2A germline mutations, accompanied with loss of heterogeneity of the wild-type allele, in a small fraction of young NSND ${ }^{41}$.

The 5-year OS of HNSCC patients in general is $40-50 \%$, whereas it is $70-80 \%$ for patients with HPV-positive OPSCC ${ }^{2,42}$. In this study, there was no significant difference in OS or DFS between virus positive and virus negative patients. However, it is not certain if the survival comparison of HPV-positive versus HPV-negative HNSCC was one of HPV-positivity or of tumor location (OPSCC versus non-OPSCC), as in this cohort HPV-negative tumors were exclusively non-OPSCC. The same applies to EBV-positive tumors, which were all NPSCC. Therefore, these survival analyses should be interpreted with caution. The 5 -year OS of $67 \%$ for patients with HPV-positive tumors was lower than expected, but these patients were relatively old with a median age of 67.3 years. Patients with HPV-negative HNSCC (53\%) did not differ significantly in 5-year OS from patients with HPV-positive OPSCC, even though they had a median age of almost 
9 years older than the patients with HPV-positive tumors (76.2 versus 67.3 years). So, considering their age at the time of HNSCC diagnosis, the HPV-negative NSND, mainly with OSCC, had a relatively good 5-year OS. Nevertheless, a young age at the time of cancer diagnosis was predictive of a better OS in both the virus negative OSCC group and the whole NSND cohort, besides a T1 stage and a N0 or N1 stage.

Viral association in all four NPSCC patients of this cohort was as expected. Although the worldwide incidence of EBV related NPSCC has been decreasing over the past decade, the prevalence is still high with almost $100 \%$ in endemic regions (southern China, Southeast Asia, Northern Africa, and the Mediterranean basin), and 60-85\% in nonendemic regions ${ }^{9,43-46}$. NPSCC infections with HPV are less common, and have been reported in studies from non-endemic regions like West Africa and Europe (outside the Mediterranean basin), with a prevalence between 1.6-16\% 43, 45, 47, 48. Based on 517 U.S. NPSCC patients with known HPV testing (34.8\% HPV-positive) in the Surveillance, Epidemiology and End Results database, predictors for HPV-positivity in NPSCC have been established: being younger than 25-years-old, Caucasian (rather than East-Asian or other ethnicities), an AJCC-7 stage other than stage 1, and no distant metastases (M0) ${ }^{49}$. Indeed, the one patient in our cohort with HPV-positive NPSCC was a Caucasian patient with stage 2 disease.

Expression of cell cycle proteins p16 and pRb was as expected in the HPV-positive OPSCC of this cohort, with overexpression of the former and loss of the latter. Although p53 is usually degraded by HPV's viral oncoprotein E6, only two HPV-positive tumors showed 0 -mutant type p53 expression, whereas the other 10 showed wild-type p53 expression. This is in agreement with earlier observations, where there was $\mathrm{p} 53$ expression in 7/10 HPV-positive tumors of non-smokers, despite absence of TP53 mutations ${ }^{50}$. Possible explanations for this finding are virally induced processes, such as hypoxia, oxidative stress, or impaired repair of double strand DNA breaks ${ }^{50,51}$. On the other hand, up to $55-75 \%$ of HPV-negative OSCC contain TP53 mutations, which is comparable to the $72 \%$ of HPV-negative OSCC showing mutant-type $\mathrm{p} 53$ expression (either 0 -type or overexpression) in the current study ${ }^{36,52}$. The EBV-positive tumors were all without p16 overexpression and with preserved pRb expression. Zhang and colleagues reported that the cell cycle pathway is the most deregulated pathway in NPSCC in comparison to non-tumor nasopharyngeal epithelium, with down-regulation of p16 and up-regulation of $\mathrm{pRb}{ }^{19}$. Although the precise mechanism of $\mathrm{p} 16$ inactivation by EBV in NPSCC remains unclear, it has been suggested that the Late Membrane Protein 1 of EBV could inhibit $\mathrm{p} 16$ expression and induce $\mathrm{pRb}$ phosphorylation, promoting the progression of the $\mathrm{G} 1 / \mathrm{S}$ phase ${ }^{19}$. This is in accordance with the cell cycle protein expressions found in the current study. 
No MCPyV was detected in the current study, which contrasts earlier findings. Hamiter and colleagues performed PCR using specific primers for the regulatory and LTAg of $\mathrm{MCPyV}$, followed by DNA sequence analysis to confirm viral presence in 6/21 (29\%, three were NSND) patients with OTSCC ${ }^{16}$. Although the OTSCC group in the current study was almost twice the size $(\mathrm{n}=39)$, no $\mathrm{MCPyV}$ presence was detected. Other studies report MCPyV DNA in 4-50\% of HNSCC based on quantitative real-time PCR, though with low viral loads ${ }^{14,15,17}$. This discrepancy could be the result of differences in sensitivity between the used detection methods (RNA-ISH and LTAg IHC versus DNA PCR and sequencing). However, the MCPyV presence in the literature was solely based on PCR and was not confirmed with another detection method. Nevertheless, the significance of MCPyV presence in HNSCC has yet to be determined, but our data strengthen the premise that $\mathrm{MCPyV}$ is not likely to play an important role in head and neck carcinogenesis.

HPV has its clinical relevance in routine practice as a prognostic marker in OPSCC, with a better DFS and OS, in addition to an improved radiosensitivity compared to HPV-negative OPSCC because of altered DNA repair, reduced hypoxic regions, and an increased cellular immune response ${ }^{3,53}$. With the conduction of multiple phase III deescalation trials for HPV-positive OPSCC, and the high HPV prevalence in OPSCC of NSND, the treatment of NSND may be affected in the near future ${ }^{4}$. For EBV-positive NPSCC, treatment usually consists of radiotherapy, with or without chemotherapy. Besides the anti-tumor effects of radiotherapy based on direct and indirect DNA damage, it also induces an immune response comprising of a network of immune-stimulatory and -inhibitory signals like up-regulation of immune checkpoint proteins such as PD-1/ PD-L1 ${ }^{54}$. Consequently, there are a number of clinical trials evaluating the incorporation of immune checkpoint inhibitors in the treatment of EBV-positive NPSCC ${ }^{10,54}$. Mutant p53 has been associated with resistance to chemoradiation in OSCC and an increased risk of locoregional recurrence and metastases ${ }^{55}$. Since NSND mainly have OSCC and frequent mutant-type $\mathrm{p} 53$ expression (as presented in this study), p 53 could be used to predict therapy failure in case of recurrent disease.

One of the limitations of this study was that the definition of when a patient was a non-smoker and non-drinker was collected retrospectively from their medical records. Nevertheless, a strict definition was used (for example with exclusion of patients with "sporadic" alcohol consumption), based on a standard history taking template including specific questions on any current or previous tobacco and alcohol consumption, during two separate hospital visits (Head and Neck outpatient clinic and Anesthesiology screening). Secondly, there was a small group of patients with tumors at anatomical sites other than the oral cavity (OSCC). In combination with the strict NSND definition, this resulted in no virus negative OPSCC and NPSCC. However, it has been reported that 
NSND are mainly patients with OSCC, so a small number of tumors outside the oral cavity was expected ${ }^{25,56}$. Thirdly, there might be a higher percentage of HNSCC positive for HPV DNA in this cohort, because COBAS PCR analysis was not performed on all samples. Conversely, HPV is only considered to be predictive for survival when being transcriptionally active, and since all tumors were tested on HPV-16/18 mRNA and p16 IHC, we expect to have detected the tumors with biologically active HPV infection ${ }^{7,8}$. Finally, some of the FFPE material was rather old ( $>25$ years of storage), which is known to often result in breakdown of the nucleic acids. Indeed, DNA quality of DNA extracted from one tumor was insufficient for COBAS analysis. Nevertheless, the TMA blocks were freshly sectioned before ISH, IHC, or PCR, and all the positive controls were adequate.

\section{CONCLUSION}

A high prevalence of HPV and EBV was observed in OPSCC and NPSCC of NSND respectively, but not in HNSCC outside these locations. Although a significant role of oncogenic viruses would be assumed in this specific patient group lacking the traditional risk factors for developing HNSCC, HPV, EBV, and MCPyV were not detected in this relatively large cohort of 95 OSCC apart from one case, using clinically relevant cutoff values. This argues against a central role of these viruses in the etiopathogenesis of oral, hypopharyngeal, and laryngeal squamous cell carcinoma in this specific patient group. With ongoing de-escalation trials for HPV-positive OPSCC and trials for immune checkpoint inhibitors in the treatment of EBV-positive NPSCC, the treatment of NSND with tumors at those locations may change in the near future. However, for the majority of NSND with virus negative OSCC, more research is needed to understand the carcinogenic mechanisms in order to consider targeted therapeutic options.

\section{Data Availability Statement}

The raw data supporting the conclusions of this article will be made available by the authors, without undue reservation.

\section{Ethics Statement}

The studies involving human participants were reviewed and approved by Maastricht University Medical Center (METc 2018-0567). Written informed consent for participation was not required for this study in accordance with the national legislation and the institutional requirements.

\section{Author Contributions}

All authors have contributed substantially to the conception and design (FM, SW, BK, and $\mathrm{ES}$ ), acquisition of data (FM, FK, FJ, and FF), analysis and interpretation of data 
(FM, SW, RB, AH, MH, BK, and ES); drafting the article (FM, FJ, and ES) or revising it critically for important intellectual content (FK, FF, SW, RB, AH, MH, and BK); final approval of the version to be published (FM, FK, FJ, FF, SW, RB, AH, MH, BK, and ES); and agree to be accountable for all aspects of the work in ensuring that questions related to the accuracy or integrity of any part of the work are appropriately investigated and resolved. All authors contributed to the article and approved the submitted version.

\section{Conflict of Interest}

SW receives a research grant from Roche, MSD, AstraZeneca, Pfizer, Nextcure, Amgen, and Bayer. BK and ES receive a research grant from Pfizer and Novartis. The funders had no role in the design of the study, in the collection, analyses, or interpretation of the data, in the writing of the manuscript, or in the decision to publish the results.

The remaining authors declare that the research was conducted in the absence of any commercial or financial relationships that could be construed as a potential conflict of interest.

\section{Acknowledgments}

We would like to thank Andrea Ruland, Robin Jacobs, and Wouter Gerritsen, Department of Pathology, Maastricht University Medical Center, Netherlands, for their assistance with the viral analyses. 


\section{References}

1. Brierley JDG, Gospodarowicz MK, Wittekind C. TNM Classification of Malignant Tumours 8th Edition. New Jersey: Wiley-Blackwell (2016).

2. O’Rorke MA, Ellison MV, Murray LJ, Moran M, James J, Anderson LA. Human papillomavirus related head and neck cancer survival: a systematic review and meta-analysis. Oral Oncol (2012) 48(12):1191-201. doi: 10.1016/j.oraloncology.2012.06.019

3. Ragin CC, Taioli E. Survival of squamous cell carcinoma of the head and neck in relation to human papillomavirus infection: review and meta-analysis. Int J Cancer (2007) 121(8):1813-20. doi: 10.1002/ijc. 22851

4. Mirghani H, Blanchard P. Treatment de-escalation for HPV-driven oropharyngeal cancer: Where do we stand? Clin Trans Radiat Oncol (2018) 8:4-11. doi: 10.1016/j.ctro.2017.10.005

5. Castellsague X, Alemany L, Quer M, Halec G, Quiros B, Tous S, et al. HPV Involvement in Head and Neck Cancers: Comprehensive Assessment of Biomarkers in 3680 Patients. J Natl Cancer Institute (2016) 108(6):djv403. doi: 10.1093/jnci/djv403

6. Hong A, Lee CS, Jones D, Veillard AS, Zhang M, Zhang X, et al. Rising prevalence of human papillomavirus-related oropharyngeal cancer in Australia over the last 2 decades. Head Neck (2016) 38(5):743-50. doi: 10.1002/hed.23942

7. Jung AC, Briolat J, Millon R, de Reynies A, Rickman D, Thomas E, et al. Biological and clinical relevance of transcriptionally active human papillomavirus (HPV) infection in oropharynx squamous cell carcinoma. Int J Cancer (2010) 126(8):1882-94. doi: 10.1002/ijc.24911

8. Mena M, Taberna M, Tous S, Marquez S, Clavero O, Quiros B, et al. Double positivity for HPVDNA/p16ink4a is the biomarker with strongest diagnostic accuracy and prognostic value for human papillomavirus related oropharyngeal cancer patients. Oral Oncol (2018) 78:137-44. doi: 10.1016/j. oraloncology.2018.01.010

9. Chan JK. Virus-associated neoplasms of the nasopharynx and sinonasal tract: diagnostic problems. Modern Pathol (2017) 30(s1):S68-s83. doi: 10.1038/modpathol.2016.189

10. Outh-Gauer S, Alt M, Le Tourneau C, Augustin J, Broudin C, Gasne C, et al. Immunotherapy in head and neck cancers: A new challenge for immunologists, pathologists and clinicians. Cancer Treat Rev (2018) 65:54-64. doi: 10.1016/j.ctrv.2018.02.008

11. Yu F, Lu Y, Petersson F, Wang DY, Loh KS. Presence of lytic Epstein-Barr virus infection in nasopharyngeal carcinoma. Head Neck (2018) 40(7):1515-23. doi: 10.1002/hed.25131

12. Feng H, Shuda M, Chang Y, Moore PS. Clonal integration of a polyomavirus in human Merkel cell carcinoma. Sci (N Y NY) (2008) 319(5866):1096-100. doi: 10.1126/science.1152586

13. Kassem A, Schopflin A, Diaz C, Weyers W, Stickeler E, Werner M, et al. Frequent detection of Merkel cell polyomavirus in human Merkel cell carcinomas and identification of a unique deletion in the VP1 gene. Cancer Res (2008) 68(13):5009-13. doi: 10.1158/0008-5472.CAN-08-0949

14. Tanio S, Matsushita M, Kuwamoto S, Horie Y, Kodani I, Murakami I, et al. Low prevalence of Merkel cell polyomavirus with low viral loads in oral and maxillofacial tumours or tumour-like lesions from 
immunocompetent patients: Absence of Merkel cell polyomavirus-associated neoplasms. Mol Clin Oncol (2015) 3(6):1301-6. doi: 10.3892/mco.2015.629

15. Salakova M, Koslabova E, Vojtechova Z, Tachezy R, Sroller V. Detection of human polyomaviruses MCPyV, HPyV6, and HPyV7 in malignant and non-malignant tonsillar tissues. J Med Virol (2016) 88(4):695-702. doi: 10.1002/jmv.24385

16. Hamiter M, Asarkar A, Rogers D, Moore-Medlin T, McClure G, Ma X, et al. A pilot study of Merkel cell polyomavirus in squamous cell carcinoma of the tongue. Oral Oncol (2017) 74:111-4. doi: 10.1016/j.oraloncology.2017.09.021

17. Mohebbi E, Noormohamadi Z, Sadeghi-Rad H, Sadeghi F, Yahyapour Y, Vaziri F, et al. Low viral load of Merkel cell polyomavirus in Iranian patients with head and neck squamous cell carcinoma: Is it clinically important? J Med Virol (2018) 90(2):344-50. doi: 10.1002/jmv.24953

18. Angiero F, Gatta LB, Seramondi R, Berenzi A, Benetti A, Magistro S, et al. Frequency and role of HPV in the progression of epithelial dysplasia to oral cancer. Anticancer Res (2010) 30(9):3435-40.

19. Zhang W, Zeng Z, Zhou Y, Xiong W, Fan S, Xiao L, et al. Identification of aberrant cell cycle regulation in Epstein-Barr virus-associated nasopharyngeal carcinoma by cDNA microarray and gene set enrichment analysis. Acta Biochim Biophys Sin (Shanghai) (2009) 41(5):414-28. doi: 10.1093/ abbs/gmp025

20. Harms PW, Harms KL, Moore PS, DeCaprio JA, Nghiem P, Wong MKK, et al. The biology and treatment of Merkel cell carcinoma: current understanding and research priorities. Nat Rev Clin Oncol (2018) 15(12):763-76. doi: 10.1038/s41571-018-0103-2

21. Tornesello ML, Annunziata C, Tornesello AL, Buonaguro L, Buonaguro FM. Human Oncoviruses and p53 Tumor Suppressor Pathway Deregulation at the Origin of Human Cancers. Cancers (2018) 10(7):213. doi: 10.3390/cancers 10070213

22. Andrews E, Seaman WT, Webster-Cyriaque J. Oropharyngeal carcinoma in non-smokers and nondrinkers: a role for HPV. Oral Oncol (2009) 45(6):486-91. doi: 10.1016/j.oraloncology.2008.07.008

23. Farshadpour F, Konings S, Speel EJ, Hordijk GJ, Koole R, van Blokland M, et al. Human Papillomavirus and Oropharyngeal Squamous Cell Carcinoma: A Case-Control Study regarding Tobacco and Alcohol Consumption. Pathol Res Int (2011) 2011:806345. doi: 10.4061/2011/806345

24. Laco J, Vosmikova H, Novakova V, Celakovsky P, Dolezalova H, Tucek L, et al. The role of high-risk human papillomavirus infection in oral and oropharyngeal squamous cell carcinoma in non-smoking and non-drinking patients: a clinicopathological and molecular study of 46 cases. Virchows Archiv An Int J Pathol (2011) 458(2):179-87. doi: 10.1007/s00428-010-1037-y

25. Mulder FJ, Pierssens D, Baijens LWJ, Kremer B, Speel EM. Evidence for different molecular parameters in head and neck squamous cell carcinoma of nonsmokers and nondrinkers: Systematic review and meta-analysis on HPV, p16, and TP53. Head Neck (2020) 41(1):303-22. doi: 10.1002/hed.26513

26. Farshadpour F, Hordijk GJ, Koole R, Slootweg PJ. Head and neck squamous cell carcinoma in nonsmoking and non-drinking patients with multiple tumors: etiologic significance of p53 and Ki-67 in non-tumorous epithelium. J Oral Pathol Med Off Publ Int Assoc Oral Pathol Am Acad Oral Pathol (2008) 37(9):549-54. doi: 10.1111/j.1600-0714.2008.00657.x 
27. Chteinberg E, Sauer CM, Rennspiess D, Beumers L, Schiffelers L, Eben J, et al. Neuroendocrine Key Regulator Gene Expression in Merkel Cell Carcinoma. Neoplasia (2018) 20(12):1227-35. doi: 10.1016/j.neo.2018.10.003

28. Mooren JJ, Gultekin SE, Straetmans JM, Haesevoets A, Peutz-Kootstra CJ, Huebbers CU, et al. P16(INK4A) immunostaining is a strong indicator for high-risk-HPV-associated oropharyngeal carcinomas and dysplasias, but is unreliable to predict low-risk-HPV-infection in head and neck papillomas and laryngeal dysplasias. Int J Cancer (2014) 134(9):2108-17. doi: 10.1002/ijc.28534

29. Li Y, Zhang J. Expression of mutant p53 in oral squamous cell carcinoma is correlated with the effectiveness of intra-arterial chemotherapy. Oncol Lett (2015) 10(5):2883-7. doi: 10.3892/ ol.2015.3651

30. Plath M, Broglie MA, Forbs D, Stoeckli SJ, Jochum W. Prognostic significance of cell cycle-associated proteins p16, pRB, cyclin D1 and p53 in resected oropharyngeal carcinoma. J Otolaryngol Head Neck Surg (2018) 47(1):53. doi: 10.1186/s40463-018-0298-3

31. Leroux-Kozal V, Leveque N, Brodard V, Lesage C, Dudez O, Makeieff M, et al. Merkel cell carcinoma: histopathologic and prognostic features according to the immunohistochemical expression of Merkel cell polyomavirus large T antigen correlated with viral load. Hum Pathol (2015) 46(3):443-53. doi: 10.1016/j.humpath.2014.12.001

32. Brägelmann J, Dagogo-Jack I, El Dinali M, Stricker T, Brown CD, Zuo Z, et al. Oral cavity tumors in younger patients show a poor prognosis and do not contain viral RNA. Oral Oncol (2013) 49(6):52533. doi: 10.1016/j.oraloncology.2013.02.003

33. Braakhuis BJ, Rietbergen MM, Buijze M, Snijders PJ, Bloemena E, Brakenhoff RH, et al. TP53 mutation and human papilloma virus status of oral squamous cell carcinomas in young adult patients. Oral Dis (2014) 20(6):602-8. doi: 10.1111/odi.12178

34. Ni Y, Zhang X, Wan Y, Dun Tang K, Xiao Y, Jing Y, et al. Relationship between p16 expression and prognosis in different anatomic subsites of OSCC. Cancer Biomark (2019) 26(3):375-83. doi: 10.3233/CBM-192402

35. Yang LQ, Xiao X, Li CX, Wu WY, Shen XM, Zhou ZT, et al. Human papillomavirus genotypes and p16 expression in oral leukoplakia and squamous cell carcinoma. Int J Clin Exp Pathol (2019) 12(3):1022-8.

36. The Cancer Genome Atlas Network. Comprehensive genomic characterization of head and neck squamous cell carcinomas. Nature (2015) 517(7536):576-82. doi: 10.1038/nature14129

37. Lechner M, Chakravarthy AR, Walter V, Masterson L, Feber A, Jay A, et al. Frequent HPV-independent p16/INK4A overexpression in head and neck cancer. Oral Oncol (2018) 83:32-7. doi: 10.1016/j. oraloncology.2018.06.006

38. Rooper LM, Windon MJ, Hernandez T, Miles B, Ha PK, Ryan WR, et al. HPV-positive Squamous Cell Carcinoma of the Larynx, Oral Cavity, and Hypopharynx: Clinicopathologic Characterization With Recognition of a Novel Warty Variant. Am J Surg Pathol (2020) 44(5):691-702. doi: 10.1097/ PAS.0000000000001433

39. Fakhry C, Lacchetti C, Rooper LM, Jordan RC, Rischin D, Sturgis EM, et al. Human Papillomavirus Testing in Head and Neck Carcinomas: ASCO Clinical Practice Guideline Endorsement of the 
College of American Pathologists Guideline. J Clin Oncol (2018) 36(31):3152-61. doi: 10.1200/ JCO.18.00684

40. Boy S, Van Rensburg EJ, Engelbrecht S, Dreyer L, van Heerden M, van Heerden W. HPV detection in primary intra-oral squamous cell carcinomas-commensal, aetiological agent or contamination? J Oral Pathol Med Off Publ Int Assoc Oral Pathol Am Acad Oral Pathol (2006) 35(2):86-90. doi: 10.1111/j.1600-0714.2006.00385.x

41. Fostira F, Koutsodontis G, Vagia E, Economopoulou P, Kotsantis I, Sasaki C, et al. Predisposing Germline Mutations in Young Patients With Squamous Cell Cancer of the Oral Cavity. JCO Precis Oncol (2018) 2):1-8. doi: 10.1200/PO.18.00022

42. Leemans CR, Braakhuis BJ, Brakenhoff RH. The molecular biology of head and neck cancer. Nat Rev Cancer (2011) 11(1):9-22. doi: 10.1038/nrc2982

43. Svajdler M Jr., Kaspirkova J, Mezencev R, Laco J, Torday T, Dubinsky P, et al. Human papillomavirus and Epstein-Barr virus in nasopharyngeal carcinoma in a non-endemic eastern european population. Neoplasma (2016) 63(1):107-14. doi: 10.4149/neo_2016_013

44. Tang LL, Chen WQ, Xue WQ, He YQ, Zheng RS, Zeng YX, et al. Global trends in incidence and mortality of nasopharyngeal carcinoma. Cancer Lett (2016) 374(1):22-30. doi: 10.1016/j. canlet.2016.01.040

45. Ruuskanen M, Irjala H, Minn H, Vahlberg T, Randen-Brady R, Hagstrom J, et al. Epstein-Barr virus and human papillomaviruses as favorable prognostic factors in nasopharyngeal carcinoma: A nationwide study in Finland. Head Neck (2019) 41(2):349-57. doi: 10.1002/hed.25450

46. Chen YP, Chan ATC, Le QT, Blanchard P, Sun Y, Ma J. Nasopharyngeal carcinoma. Lancet (2019) 394(10192):64-80. doi: 10.1016/S0140-6736(19)30956-0

47. Asante DB, Asmah RH, Adjei AA, Kyei F, Simpong DL, Brown CA, et al. Detection of Human Papillomavirus Genotypes and Epstein-Barr Virus in Nasopharyngeal Carcinomas at the Korle-Bu Teaching Hospital, Ghana. ScientificWorldJournal (2017) 2017:2721367. doi: 10.1155/2017/2721367

48. Robinson M, Suh YE, Paleri V, Devlin D, Ayaz B, Pertl L, et al. Oncogenic human papillomavirusassociated nasopharyngeal carcinoma: an observational study of correlation with ethnicity, histological subtype and outcome in a UK population. Infect Agent Cancer (2013) 8(1):30. doi: 10.1186/17509378-8-30

49. Wotman M, Oh EJ, Ahn S, Kraus D, Costantino P, Tham T. HPV status in patients with nasopharyngeal carcinoma in the United States: A SEER database study. Am J Otolaryngol (2019) 40(5):705-10. doi: 10.1016/j.amjoto.2019.06.007

50. Hafkamp HC, Speel EJ, Haesevoets A, Bot FJ, Dinjens WN, Ramaekers FC, et al. A subset of head and neck squamous cell carcinomas exhibits integration of HPV 16/18 DNA and overexpression of p16INK4A and p53 in the absence of mutations in p53 exons 5-8. Int J Cancer (2003) 107(3):394400. doi: 10.1002/ijc.11389

51. Verhees F, Legemaate D, Demers I, Jacobs R, Haakma WE, Rousch M, et al. The Antiviral Agent Cidofovir Induces DNA Damage and Mitotic Catastrophe in HPV-Positive and -Negative Head and Neck Squamous Cell Carcinomas In Vitro. Cancers (2019) 11(7):919. doi: 10.3390/cancers11070919 
52. Azulay EE, Cooks T, Elkabets M. Potential oncogenic roles of mutant-p53-derived exosomes in the tumor-host interaction of head and neck cancers. Cancer Immunol Immunother (2020) 69(2):285-92. doi: 10.1007/s00262-019-02450-5

53. Liu C, Mann D, Sinha UK, Kokot NC. The molecular mechanisms of increased radiosensitivity of HPV-positive oropharyngeal squamous cell carcinoma (OPSCC): an extensive review. J Otolaryngol Head Neck Surg (2018) 47(1):59. doi: 10.1186/s40463-018-0302-y

54. Yeo ELL, Li YQ, Soo KC, Wee JTS, Chua MLK. Combinatorial strategies of radiotherapy and immunotherapy in nasopharyngeal carcinoma. Chin Clin Oncol (2018) 7(2):15. doi: 10.21037/ cco.2018.04.05

55. Gupta S, Kushwaha VS, Verma S, Khan H, Bhatt ML, Husain N, et al. Understanding molecular markers in recurrent oral squamous cell carcinoma treated with chemoradiation. Heliyon (2016) 2(12):e00206. doi: 10.1016/j.heliyon.2016.e00206

56. Farshadpour F, Hordijk GJ, Koole R, Slootweg PJ. Non-smoking and non-drinking patients with head and neck squamous cell carcinoma: a distinct population. Oral Dis (2007) 13(2):239-43. doi: 10.1111/j.1601-0825.2006.01274.x 


\section{Supplementary data:}

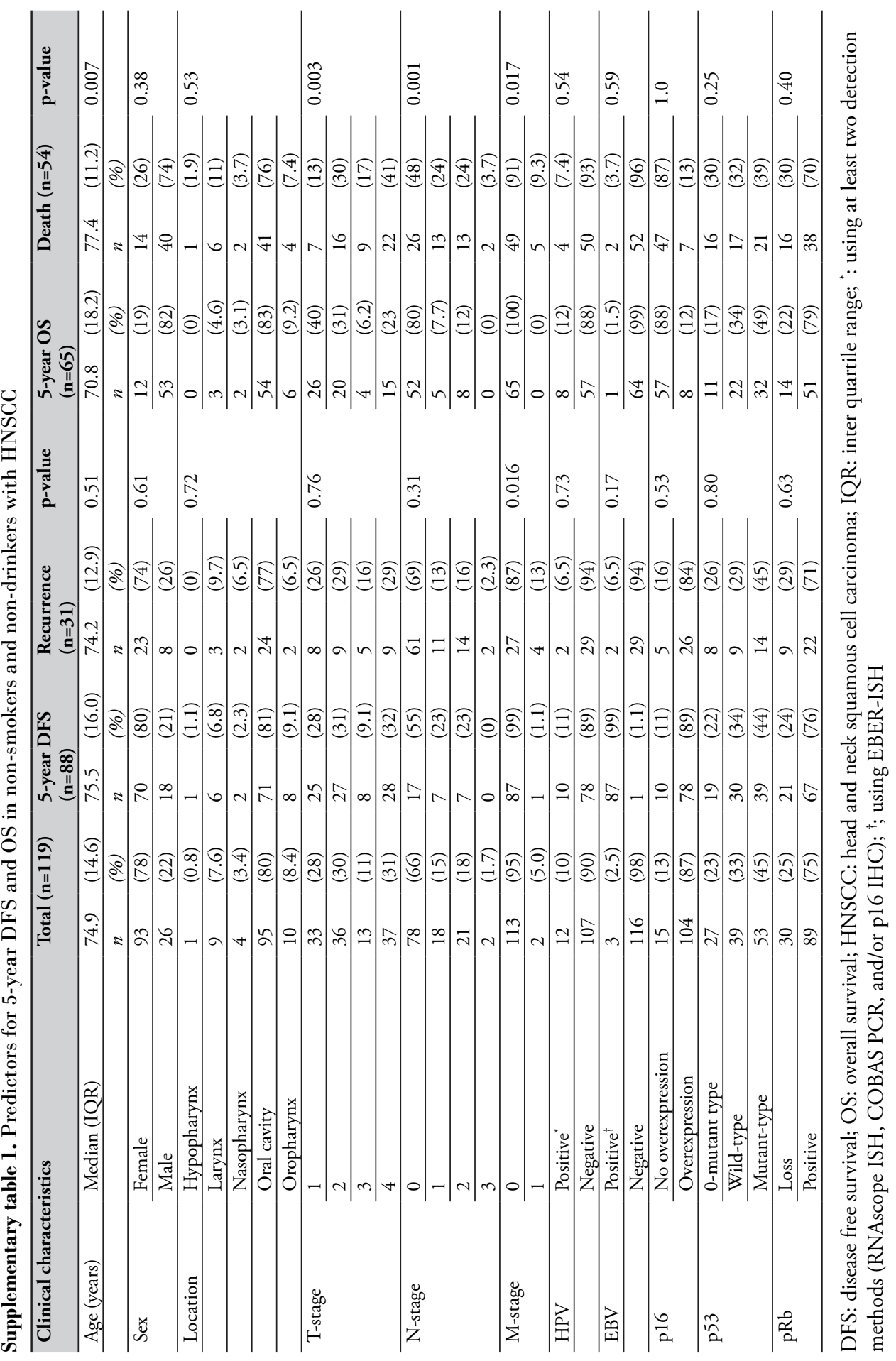




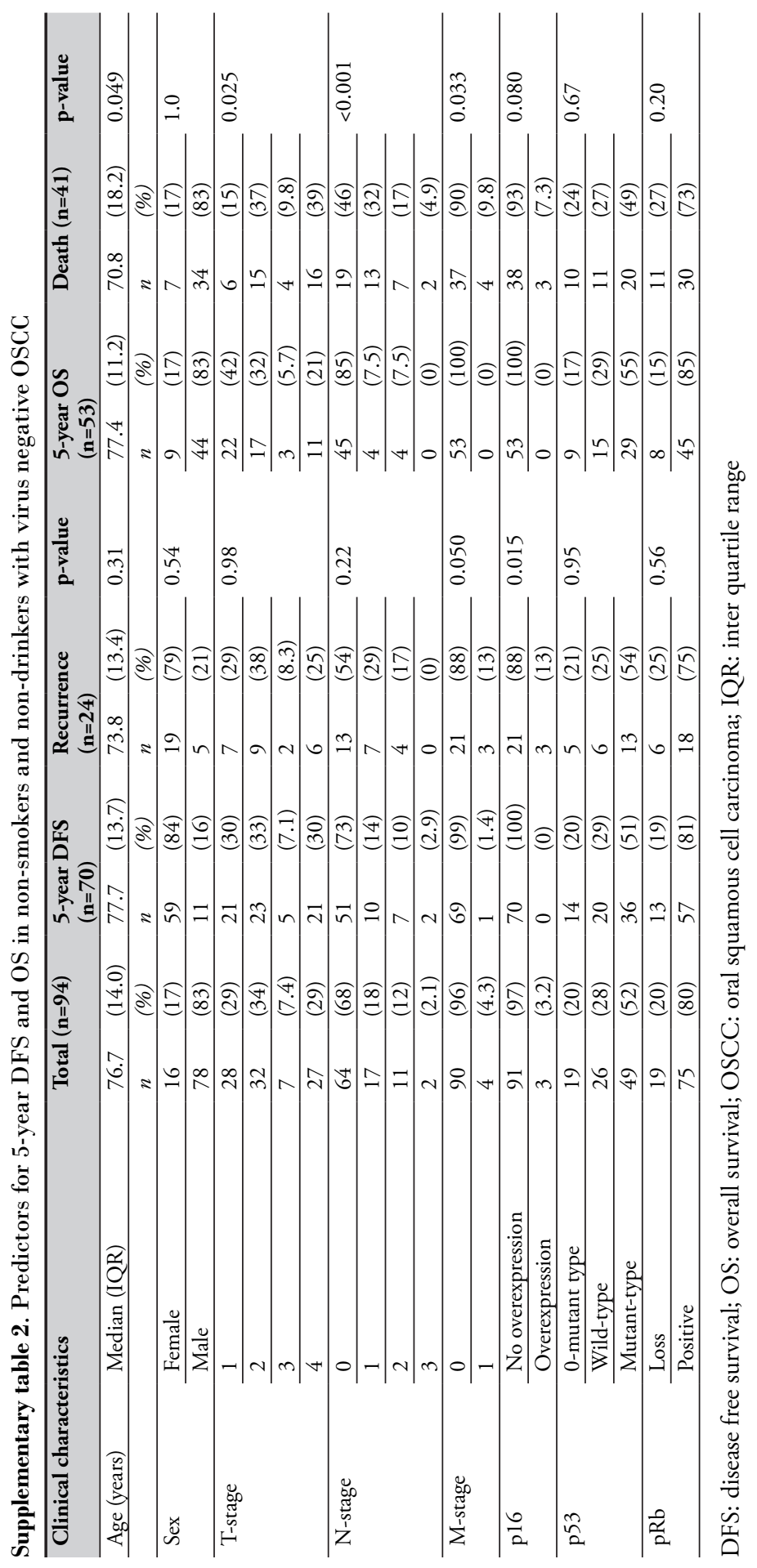


CHAPTER 7

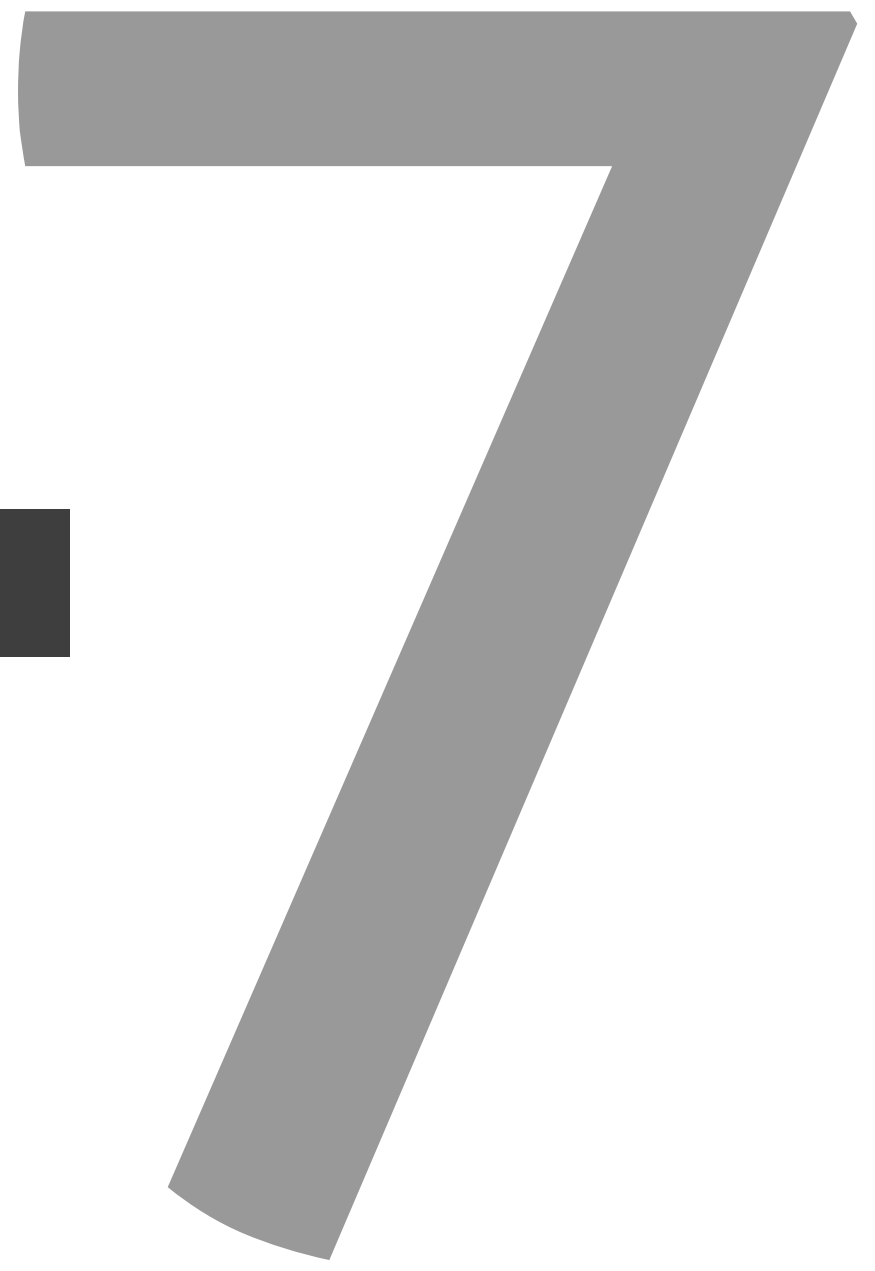


General discussion 
Cancer is still a crucial public health concern accounting for nearly 9.6 million deaths worldwide in $2018{ }^{1}$. The overall cancer death rate remains high and this reflects to several risk factors including the increase of the population and the life expectancy ${ }^{1}$. However, the etiology of many cancers is still unknown. To date, it is estimated that up to $20 \%$ of the world's cancer cases burden is attributed to infectious pathogens ${ }^{1-4}$. In the past years, studies have shown that viral infection plays a significant role in the carcinogenesis of $\sim 10$ $15 \%$ of all human cancers worldwide ${ }^{5-7}$. In the context of cancer patient management, the discovery of novel virus-associated human cancers provides an important aspect in understanding the mechanism of tumorigenesis, which will result in the improvement of knowledge about prevention and treatment. Given the potentially serious implications of human viruses in tumorigenesis, it is important to elucidate further evidence for the involvement of additional viruses in human carcinogenesis.

Infection with polyomaviruses (PyVs) comes with a long history of controversy about its role in human oncogenesis. PyVs and papillomaviruses (HPVs) used to belong to the same family of Papovaviridae since both viruses are small non-enveloped with doublestranded DNA genomes and sharing some genetic organization and viral replication mechanisms ${ }^{8-10}$. According to the ICTV 7th Report in 2000, Papovaviridae has been split into two new families, PyVs assigned to Polyomavirdae family while HPVs assigned to Papillomaviridae ${ }^{11}$. Thus, it is speculated that PyVs are possibly involved in human tumorigenesis as an -analogous to HPVs. Until 2007, BK polyomavirus (BKPyV) and JC polyomavirus $(\mathrm{JCPyV})$ were the only known $\mathrm{HPyVs}$, both revealing a high seroprevalence in healthy individuals and possibly causing serious diseases in immunocompromised subjects ${ }^{12,13}$. Between 2007 and 2019, 13 new PyVs have been isolated from human specimens as outlined in chapter 1 (Table 2) and 9 of them have been confirmed to be HPyVs ${ }^{10,14-16}$. In general, it seems that the infection of HPyVs is asymptomatic, but might reactivate and lead to diseases in immunocompromised individuals. Moreover, there are strong evidence suggesting that some of $\mathrm{HPyV}$ s have the ability to transform cells in animal models. For instance, epithelium tumor developed in transgenic mice after injecting them with the early region gene (tumor antigen region) of $\mathrm{BKPyV}$, while the injection of JCPyV early region gene initiated neural tumor formation in these mice ${ }^{17}, 18$. In addition, JCPyV showed to be able to induce brain tumors in rats and hamsters ${ }^{19-22}$. Nevertheless, except for MCPyV, none of the new HPyVs has yet been implicated in human tumorigenesis.

$\mathrm{MCPyV}$ is the only $\mathrm{HPyV}$ that has been categorized as a "Group 2A carcinogen: probably carcinogenic to humans" by the International Agency for Research on Cancer (IARC) in $2012^{23}$. These days, almost over 10 years later of additional evidence of finding MCPyV-DNA clonally integrated into highly malignant non-melanoma skin cancer Merkel cell carcinoma (MCC) tumor host genome with no UV mutational signature, it 
seems very likely that MCPyV would be classified as "Group 1 carcinogen: Carcinogenic to humans" ${ }^{24-28}$. MCPyV-positive MCC have been reported to have a relatively favorable clinical outcome, drug response, and survival rate compared to MCPyV-negative MCC. The etiopathogenesis of MCPyV-negative MCC is closely linked to UV damage and is characterized by a very high tumor mutational burden ${ }^{29-33}$. The rapidly expanding number of $\mathrm{HPyV}$ members raises the question whether these viruses may also be involved in the etiopathogenesis of human diseases, including malignant tumors. The discovery of MCPyV and its role as a major etiopathogenetic agent of MCC broadly renewed the research interest in $\mathrm{HPyV}$-associated tumorigenesis. To this end, researchers throughout the world have studied the prevalence of HPyVs in a variety of diseases ${ }^{10,34-39}$.

This thesis aims to gain more insight into the possible involvement of known HPyVs in the tumorigenesis of a variety of human cancers. The presence of HPyVs and their association with human malignancies have commonly been tested by PCR, and only a very limited number of studies showed the presence of these viruses at the single-cell level within the histomorphological context chapter 2. The detection of viral-DNA in tissue extracts does not prove a possible role of $\mathrm{HPyVs}$ in cancer, because it cannot be interpreted in which cellular compartment the viral DNA is present. Viral protein expression as assessed by IHC in FFPE tissues is mostly hampered by the limited number of virus-specific, commercially available antibodies. Based on these limitations we tried to utilize other molecular techniques such as FISH, RISH, and IHC to show the viral DNA, RNA, and protein expression of the respective $\mathrm{HPyV}$ in a histomorphological context as demonstrated in chapter 3, 5, and 6. By doing so, we have added novel data about the potential role of the tested HPyVs and their involvement in human diseases, including their latency. Recently, next-generation sequencing (NGS) technology has helped in discovering novel members of PyVs such as QPyV ${ }^{40,41}$. NGS is not only an important approach to determine the presence and the sequences of the virus but also to identify their sites of integration in the human tumor genome. In the nearby future, employing new emerging approaches combining NGS technology with bioinformatics will be beneficial for analyzing FFPE archival tissue for virus integration ${ }^{42}$.

To date, five HPyVs have been isolated from the human skin of asymptomatic individuals (i.e., MCPyV, HPyV6, HPyV7, TSPyV, and HPyV9) chapter 1. Although it has been reported that $\mathrm{HPyV} 6$ and 7 appear to be associated with pruritic dermal dyskeratotic dermatoses ${ }^{43,44}$, further associations of these viruses with clinical diseases have not been studied intensely. In chapter $\mathbf{2}$ we conducted the first comprehensive review to provide an insight into the emerging role of these two closely related HPyVs in human cancers. The frequent prevalence of HPyV6 and 7 DNA in non-neoplastic and neoplastic tissues along with their high seropositivity in the normal population indicates that both viruses have long-term latency in humans. Therefore, HPyV6 and 7 are putative candidates that 
may contribute to the etiopathogenesis of human diseases, especially skin tumors. It could be concluded that the detection frequency of HPyV6 and 7 is higher in malignant tissues than in non-neoplastic tissues. Interestingly, HPyV6 prevalence was especially high in cutaneous malignancies, whereas only a low frequency of these tumors harbored HPyV7. In contrast, HPyV7 was more frequently detected in other malignancies such as cholangiocarcinoma, which is described in chapter 3. In addition, by summarizing the age-specific sero-epidemiological studies of HPyV6 and 7, it was found that both viruses infect individuals at an early age, and HPyV6 seroprevalence was found to be slightly higher than $\mathrm{HPyV7}$ and increased with age ${ }^{16,45-52}$. Very limited studies reported the seroprevalence of $\mathrm{HPyV} 6$ and 7 in specimens of cancer patients, and the seropositivity was found to be comparable to those in healthy population age groups ${ }^{51,52}$.

Cholangiocarcinoma (CCA) is rare biliary duct neoplasia with a very poor prognosis and the etiology of CCA remains largely unknown ${ }^{53}$. Few infectious agents have been identified to be associated with the etiopathogenesis of CCA such as hepatitis B and C viruses ${ }^{53-55}$. Interestingly, a recent study by Chan $e t$ al. research group investigated the presence of HPyVs in the bile fluid of patients with a variety of hepatobiliary malignancies and they reported that HPyV6 but not HPyV7 was detected in bile specimens from CCA patients ${ }^{56}$. Therefore, we investigated the prevalence and possible pathological associations of HPyVs with patients diagnosed with CCA in chapter 3. The FFPE tissues obtained from the CCA cohort provided a unique opportunity to examine the prevalence of HPyVs not only in CCA tumor tissues, but also the adjacent non-neoplastic bile duct epithelium, and non-lesional hepatocytes ${ }^{57}$. In addition, a sensitive degenerated PCR primer set was designed to simultaneously detect highly conserved sequences in the LTAg gene of all currently known HPyVs. As a result, HPyV7, HPyV6, and MCPyV were commonly detected in the FFPE of malignant, premalignant, and non-lesional CCA using degenerated PCR and subsequent sequencing. In summary, HPyV7 (69\%) was the most prevalent $\mathrm{HPyV}$ in the tested cohort, followed by MCPyV (24\%), and HPyV6 (14\%). Interestingly, co-detection of two HPyVs was common, i.e. eight cases showed coinfection with HPyV7 and MCPyV, and two cases with HPyV6 and HPyV7. These findings are slightly different than those from the previous study by Chan et. al. ${ }^{56}$. The most likely explanation for this difference between high $\mathrm{HPyV} 7$ prevalence in CCA found in this thesis and high HPyV6 prevalence found by Chan et al. is the primary source of the DNA, i.e. primary CCA tissues versus bile fluids of CCA patients, respectively. In addition, the findings might reflect geographical differences in the prevalence of HPyV6 and 7. In conclusion, the review presented in chapter 2 reveals that many screening studies for HPyVs in FFPE and fresh frozen tissue material show an elevated prevalence of $\mathrm{HPyV} 6$ and/or 7 compared to those using liquid biopsy specimens. 
Assessing the possible role of HPyVs in CCA carcinogenesis solely based on PCR results is insufficient. Additional molecular techniques are therefore indicated in chapter 2 to also show which cells harbor the respective HPyV. Upon the start of this study, the presence of known HPyVs at the single-cell level in either non-neoplastic or malignant cells of CCA and liver tissues had not been shown previously. This study thus is the first to localize HPyV6, HPyV7, and MCPyV in the bile duct epithelium and hepatocytes using FISH, IHC, and HPyV6 transcripts by RNA-ISH. Altogether, the results strongly indicate that three HPyVs (HPyV7, HPyV6, and MCPyV) are hepatotropic viruses and may infect non-neoplastic human hepatocytes, bile duct epithelium, and CCA lesions. Yet, it remains unclear if and how HPyVs might contribute to the tumorigenesis of CCA. The frequent finding of $\mathrm{HPyVs}$, especially $\mathrm{HPyV} 7$ in $\mathrm{CCA}$ and adjacent peritumoral hepatocytes tissues, suggests an indirect role of $\mathrm{HPyV} 7$ in CCA carcinogenesis, which is possibly initiated by inflammation. For instance, hepatitis $\mathrm{B}$ and $\mathrm{C}$ viruses are known to induce neoplasms indirectly and are found in the non-malignant hepatocytes adjacent to the hepatocellular carcinoma as discussed in chapter 2.

$\mathrm{BKPyV}$ is the first identified $\mathrm{HPyV}$ and is known to be latently present in more than $50 \%$ of the population at very low levels within the epithelium of the proximal tubules of the kidney, the urothelial cell layer of the bladder, the ureters, and the renal pelvis ${ }^{58,59}$. But so far, $\mathrm{BKPyV}$ has only been found latently in the urogenital tract. BKPyV is reported to be reactivated following immunosuppression, especially in the context of kidney and solid organ transplantations, with the risk of developing $\mathrm{BKPyV}$-associated nephropathy (PyVAN) and hemorrhagic cystitis diseases ${ }^{60,61}$. Therefore, BKPyV has been suspected as a putative oncogenic virus in the development of urothelial cell carcinomas (UCC) in immunocompromised patients ${ }^{23,62}$. In chapter 4 , we focused on elucidating further evidence for the involvement of BKPyV in the UCC of the urinary bladder. In this study, the detection of BKPyV-positive urine cytology specimens (UCS) was evaluated in the $\mathrm{UCC}$ of the urinary bladder. Additionally, we assessed the relation of $\mathrm{BKPyV}$-positive UCS to a previously given intravesicular BCG or mitomycin treatment UCC patients. ${ }^{63}$. Interestingly, the overall cytology results revealed a very low detection rate $(0.2 \%)$ of Decoy cells (urothelial cells often infected with viruses, including BKPyV) within the urine cytology samples from the database of Maastricht university medical center+ institution over a 15 -year period, which is consistent with previous findings ${ }^{64,65}$. The presence of Decoy cells in the urine specimens is an indication for the reactivation of $\mathrm{BKPyV}^{66}$. Although BKPyV-IHC revealed positivity in the urine of all $(0.2 \%)$ specimens with Decoy cells. In addition, PCR detected BKPyV-DNA in the urine samples of patients diagnosed with either in situ or invasive UCC of the urinary bladder, while BKPyVIHC and PCR were negative in FFPE tissues level of primary UCCs and metastases. In summary, these results strongly indicate that the reactivation of $\mathrm{BKPyV}$ in urine cytology is not associated with prior resected ( range $1-13$ years) UCC ${ }^{63}$. Furthermore, these 
results revealed that $\mathrm{BKPyV}$-reactivation is not restricted to immunosuppression since the virus could also be detected in the urine of immunocompetent patients without any history of transplantation, malignancy, or chronic diseases. A prominent role for $\mathrm{BKPyV}$ in the etiopathogenesis of the conventional type UCC is very unlikely because one would expect a high expression of tumor-viral proteins in every tumor cell, which is not the case in the cohort subset described in chapter 3. Recently, it has been suggested that BKPyV-DNA might be lost after inducing bladder cancer development through a 'hit-and-run' mechanism similar to the model of HPV inducing skin cancer in mice ${ }^{67}$, ${ }^{68}$. However, such a mechanism proposed by Skinner in 1976 still remains speculation and lacks substantial evidence chapter ${ }^{69}$.

Thymoma is a rare intrathoracic malignancy of unknown etiology ${ }^{70}$. The major capsid protein VP-1 of murine polyomavirus was reported to induce thymomas when injected into neonatal mice ${ }^{71}$. In addition, a few years ago our research group demonstrated the high prevalence of $\mathrm{HPyV} 7$ in thymic epithelial tumors ${ }^{72}$. In chapter 5 we aimed to uncover whether $\mathrm{MCPyV}$ also plays a role in the etiopathogenesis of human epithelial thymic tumors. This was pursued by applying diverse molecular techniques to screen for MCPyV prevalence in a clinicopathologically well-characterized thymoma cohort 73. The presence of MCPyV-DNA was identified in 19.4\% of the 36 thymomas FFPE tissues by PCR in this study. Additionally, we have reported the presence of MCPyV in epithelial thymoma tissues by FISH, RISH, and IHC. The most likely conclusion is that the contribution of MCPyV to the etiopathogenesis of thymoma is unlikely at least based on our findings data which revealed less detection and expression of viral DNA, RNA, and proteins in tumor cells. As we indicated in chapter 3, one would expect to observe a pattern of high expression of viral proteins in every tumor cell as has been reported e.g. Merkel cell carcinoma positive-MCPyV ${ }^{74}$. Since this is not the case despite the presence of MCPyV-DNA, the virus might be present in a latent form in thymus tissue. Furthermore, the presence of $\mathrm{MCPyV}$ in thymoma tumors is not enough, further investigation is needed to find if $\mathrm{MCPyV}$ is integrated into the tumor genome and possibly carries tumor-specific mutations within the viral helicase of LTAg in analogy to MCC positive-MCPyV.

Human papillomavirus (HPV) is the primary cause of uterine cervical cancer but has also been shown to be involved in the development of head and neck squamous cell carcinoma (HNSCC), especially tumors originating in the oropharynx ${ }^{1}$. In addition, Epstein-Barr virus (EBV) has been found to be associated with nasopharyngeal carcinoma ${ }^{1}$, and the presence of MCPyV-DNA has been reported in oral tongue SCC and tonsillar carcinomas ${ }^{75-77}$. Generally, HPV-related HNSCC has a more favorable overall and disease-free survival than HPV-negative HNSCC ${ }^{78}$. Similarly, MCPyV-positive MCC was demonstrated to have a better prognosis than MCPyV-negative MCC ${ }^{29-33}$. It is 
proposed that the frequency of human tumor viruses might be increased in HNSCC of non-smokers and non-drinkers ${ }^{79-81}$. In chapter $\mathbf{6}$, we examined the frequency of three different tumor viruses, i.e. $\mathrm{HPV}, \mathrm{EBV}$, and $\mathrm{MCPyV}$, in a unique large cohort of non-smokers and non-drinkers with HNSCC ${ }^{82}$. Data in chapter $\mathbf{6}$ demonstrate the presence of HPV and EBV in patients with HNSCC, but MCPyV-LTAg expression was not detected by both RNA-ISH and IHC. Early results by others indicated that MCPyV-DNA was detectable in $28.6 \%$ of oral tongue SCC ${ }^{75}$. Additionally, the study of Saláková et. al. reported that MCPyV-DNA prevalence is significantly higher in tonsillar carcinomas compared to non-malignant tonsils (35.7\% versus $10.2 \%$, respectively) ${ }^{76}$. In contrast, an earlier study from 2009 revealed a low prevalence of MCPyV (3.5\%) in the tissue of non-malignant tonsils, which is not in agreement with the other two studies described above ${ }^{77}$. The discrepancy of the results described in chapter $\mathbf{6}$ with that from others is most likely due to the detection method because in chapter $\mathbf{6}$ the presence of MCPyV-LTAg expression evaluated in a single-cell level using RNA-ISH and IHC, while the other studies only tested their cohort by PCR. Moreover, a previous study revealed that the mean of MCPyV viral load was low in both malignant and non-malignant tonsils, which is different from the high MCPyV load detected in MCC ${ }^{76}$. Thus, also tonsillar tissues might be the site of MCPyV latency as speculated for $\mathrm{BKPyV}$ and JCPyV 77, 83. As a result, the findings in this thesis implicate that is unlikely $\mathrm{MCPyV}$ plays an etiological role in HNSCC tumorigenesis.

Finally, the overall results presented in this thesis lead to the conclusion that much remains to be done to elucidate the role of HPyVs as human tumor viruses. MCPyV is classified as a tumor virus for the majority of MCC cases. Also, the frequent detection of $\mathrm{HPyVs}$ in diverse tumor tissues indicates that $\mathrm{HPyVs}$ remain important putative candidates possibly contributing to human tumorigenesis. The association of HPyVs with human cancers usually has been assessed by PCR. However, the sheer presence of viral DNA is insufficient to conclude an etiopathogenic role for HPyVs. In this thesis the presence of some HPyVs was tested in the histomorphological context of selected cancer types by using diverse molecular techniques. Nevertheless, future work needs to address if these viruses are integrated into the human tumor genome and carry tumorspecific mutations as discussed in Chapter 2. Therefore, additional molecular techniques are needed to unravel if these viruses have specific interactions with the cell cycles. In particular to characterize the interaction of $\mathrm{HPyV}$ s with proteins such as p53 and pRb to possibly understand the contribution of these viruses to human cancers. 


\section{References:}

1. Wild CP WE, Stewart BW, editors. World Cancer Report: Cancer Research for Cancer Prevention. Lyon, France: International Agency for Research on Cancer Available from: http:// publicationsiarcfr/586 Licence: CC BY-NC-ND 30 IGO. 2020.

2. De Martel C, Georges D, Bray F, Ferlay J, Clifford GM. Global burden of cancer attributable to infections in 2018: a worldwide incidence analysis. The Lancet Global Health. 2020;8(2):e180-e90.

3. Bouvard V, Baan R, Straif K, Grosse Y, Secretan B, El Ghissassi F, et al. A review of human carcinogens_-Part B: biological agents. The Lancet Oncology. 2009;10(4):321-2.

4. Plummer M, De Martel C, Vignat J, Ferlay J, Bray F, Franceschi S. Global burden of cancers attributable to infections in 2012: a synthetic analysis. The Lancet Global Health. 2016;4(9):e609-e16.

5. Moore PS, Chang Y. Why do viruses cause cancer? Highlights of the first century of human tumour virology. Nature reviews Cancer. 2010;10(12):878-89.

6. Ahuja R, Jamal A, Nosrati N, Pandey V, Rajput P, Saxena N, et al. Human oncogenic viruses and cancer. Current Science. 2014;107(5):768-85.

7. zur Hausen H. Viruses in human cancers. Science (New York, NY). 1991;254(5035):1167-73.

8. White EA, Kramer RE, Hwang JH, Pores Fernando AT, Naetar N, Hahn WC, et al. Papillomavirus E7 oncoproteins share functions with polyomavirus small $\mathrm{T}$ antigens. Journal of virology. 2015;89(5):2857-65.

9. Moens U. Human Polyomaviruses and Papillomaviruses. Int J Mol Sci. 2018;19(8).

10. DeCaprio JA, Garcea RL. A cornucopia of human polyomaviruses. Nat Rev Microbiol. 2013;11(4):26476.

11. van Regenmortel MHV, Fauquet, C.M., Bishop, D.H.L., Carstens, E.B., Estes, M.K.,Lemon, S.M., Maniloff, J., Mayo, M.A., McGeoch, D.J., Pringle, C.R. and Wickner, R.B. Virus taxonomy. Seventh report of the International Committee on Taxonomy of Viruses. Academic Press, San Diego2000 [7th:[Available from: https://talk.ictvonline.org/ictv/proposals/ICTV\%207th\%20Report.pdf.

12. Bennett SM, Broekema NM, Imperiale MJ. BK polyomavirus: emerging pathogen. Microbes Infect. 2012;14(9):672-83.

13. Johnson EM, Wortman MJ, Dagdanova AV, Lundberg PS, Daniel DC. Polyomavirus JC in the context of immunosuppression: a series of adaptive, DNA replication-driven recombination events in the development of progressive multifocal leukoencephalopathy. Clin Dev Immunol. 2013;2013:197807.

14. Johne R, Buck CB, Allander T, Atwood WJ, Garcea RL, Imperiale MJ, et al. Taxonomical developments in the family Polyomaviridae. Archives of virology. 2011;156(9):1627-34.

15. Johne R, Müller H. Polyomaviruses of birds: etiologic agents of inflammatory diseases in a tumor virus family. Journal of virology. 2007;81(21):11554-9.

16. Klufah F, Mobaraki G, Liu D, Alharbi RA, Kurz AK, Speel EJM, et al. Emerging role of human polyomaviruses 6 and 7 in human cancers. Infectious agents and cancer. 2021;16(1).

17. Small JA, Khoury G, Jay G, Howley PM, Scangos GA. Early regions of JC virus and BK virus induce distinct and tissue-specific tumors in transgenic mice. Proceedings of the National Academy of Sciences of the United States of America. 1986;83(21):8288-92. 
18. Dalrymple SA, Beemon KL. BK virus $\mathrm{T}$ antigens induce kidney carcinomas and thymoproliferative disorders in transgenic mice. Journal of virology. 1990;64(3):1182-91.

19. Padgett BL, Walker DL, ZuRhein GM, Varakis JN. Differential neurooncogenicity of strains of JC virus, a human polyoma virus, in newborn Syrian hamsters. Cancer research. 1977;37(3):718-20.

20. Zu Rhein GM, Varakis JN. Perinatal induction of medulloblastomas in Syrian golden hamsters by a human polyoma virus (JC). Natl Cancer Inst Monogr. 1979(51):205-8.

21. Walker DL, Padgett BL, ZuRhein GM, Albert AE, Marsh RF. Human papovavirus (JC): induction of brain tumors in hamsters. Science (New York, NY). 1973;181(4100):674-6.

22. Ohsumi S, Motoi M, Ogawa K. Induction of undifferentiated tumors by JC virus in the cerebrum of rats. Acta pathologica japonica. 1986;36(6):815-25.

23. Bouvard V, Baan RA, Grosse Y, Lauby-Secretan B, El Ghissassi F, Benbrahim-Tallaa L, et al. Carcinogenicity of malaria and of some polyomaviruses. The Lancet Oncology. 2012;13(4):339-40.

24. Becker JC, Stang A, DeCaprio JA, Cerroni L, Lebbe C, Veness M, et al. Merkel cell carcinoma. Nat Rev Dis Primers. 2017;3:17077.

25. Kuwamoto S. Recent advances in the biology of Merkel cell carcinoma. Human pathology. 2011;42(8):1063-77.

26. Fernandez-Figueras MT, Puig L, Musulen E, Gilaberte M, Lerma E, Serrano S, et al. Expression profiles associated with aggressive behavior in Merkel cell carcinoma. Modern pathology : an official journal of the United States and Canadian Academy of Pathology, Inc. 2007;20(1):90-101.

27. Shuda M, Feng H, Kwun HJ, Rosen ST, Gjoerup O, Moore PS, et al. T antigen mutations are a human tumor-specific signature for Merkel cell polyomavirus. Proceedings of the National Academy of Sciences of the United States of America. 2008;105(42):16272-7.

28. Feng H, Shuda M, Chang Y, Moore PS. Clonal integration of a polyomavirus in human Merkel cell carcinoma. Science (New York, NY). 2008;319(5866):1096-100.

29. Eid M, Nguyen J, Brownell I. Seeking Standards for the Detection of Merkel Cell Polyomavirus and its Clinical Significance. The Journal of investigative dermatology. 2017;137(4):797-9.

30. Moshiri AS, Doumani R, Yelistratova L, Blom A, Lachance K, Shinohara MM, et al. PolyomavirusNegative Merkel Cell Carcinoma: A More Aggressive Subtype Based on Analysis of 282 Cases Using Multimodal Tumor Virus Detection. The Journal of investigative dermatology. 2017;137(4):819-27.

31. Schrama D, Peitsch WK, Zapatka M, Kneitz H, Houben R, Eib S, et al. Merkel cell polyomavirus status is not associated with clinical course of Merkel cell carcinoma. The Journal of investigative dermatology. 2011;131(8):1631-8.

32. Harms KL, Zhao L, Johnson B, Wang X, Carskadon S, Palanisamy N, et al. Virus-positive Merkel Cell Carcinoma Is an Independent Prognostic Group with Distinct Predictive Biomarkers. Clinical cancer research : an official journal of the American Association for Cancer Research. 2021;27(9):2494-504.

33. Björn Andtback H, Björnhagen-Säfwenberg V, Shi H, Lui WO, Masucci GV, Villabona L. Sex Differences in Overall Survival and the Effect of Radiotherapy in Merkel Cell Carcinoma-A Retrospective Analysis of a Swedish Cohort. Cancers. 2021;13(2).

34. zur Hausen $\mathrm{H}$. Novel human polyomaviruses-re-emergence of a well known virus family as possible human carcinogens. International journal of cancer. 2008;123(2):247-50. 
35. Buck CB, Van Doorslaer K, Peretti A, Geoghegan EM, Tisza MJ, An P, et al. The Ancient Evolutionary History of Polyomaviruses. PLoS pathogens. 2016;12(4):e1005574.

36. Prado JCM, Monezi TA, Amorim AT, Lino V, Paladino A, Boccardo E. Human polyomaviruses and cancer: an overview. Clinics (Sao Paulo, Brazil). 2018;73(suppl 1):e558s.

37. Delbue S, Comar M, Ferrante P. Review on the relationship between human polyomaviruses-associated tumors and host immune system. Clin Dev Immunol. 2012;2012:542092.

38. White MK, Khalili K. Polyomaviruses and human cancer: molecular mechanisms underlying patterns of tumorigenesis. Virology. 2004;324(1):1-16.

39. Moens U, Rasheed K, Abdulsalam I, Sveinbjornsson B. The role of Merkel cell polyomavirus and other human polyomaviruses in emerging hallmarks of cancer. Viruses. 2015;7(4):1871-901.

40. Gheit T, Dutta S, Oliver J, Robitaille A, Hampras S, Combes JD, et al. Isolation and characterization of a novel putative human polyomavirus. Virology. 2017;506:45-54.

41. Ondov BD, Starrett GJ, Sappington A, Kostic A, Koren S, Buck CB, et al. Mash Screen: highthroughput sequence containment estimation for genome discovery. Genome biology. 2019;20(1):232.

42. Balaji H, Demers I, Wuerdemann N, Schrijnder J, Kremer B, Klussmann JP, et al. Causes and Consequences of HPV Integration in Head and Neck Squamous Cell Carcinomas: State of the Art. Cancers. 2021;13(16).

43. Nguyen KD, Lee EE, Yue Y, Stork J, Pock L, North JP, et al. Human polyomavirus 6 and 7 are associated with pruritic and dyskeratotic dermatoses. Journal of the American Academy of Dermatology. 2017;76(5):932-40 e3.

44. Ho J, Jedrych JJ, Feng H, Natalie AA, Grandinetti L, Mirvish E, et al. Human polyomavirus 7 -associated pruritic rash and viremia in transplant recipients. The Journal of infectious diseases. 2015;211(10):1560-5.

45. Schowalter RM, Pastrana DV, Pumphrey KA, Moyer AL, Buck CB. Merkel cell polyomavirus and two previously unknown polyomaviruses are chronically shed from human skin. Cell host $\&$ microbe. 2010;7(6):509-15.

46. Sroller V, Hamsikova E, Ludvikova V, Musil J, Nemeckova S, Salakova M. Seroprevalence rates of HPyV6, HPyV7, TSPyV, HPyV9, MWPyV and KIPyV polyomaviruses among the healthy blood donors. Journal of medical virology. 2016;88(7):1254-61.

47. Kamminga S, van der Meijden E, Feltkamp MCW, Zaaijer HL. Seroprevalence of fourteen human polyomaviruses determined in blood donors. PloS one. 2018;13(10):e0206273.

48. Nicol JT, Robinot R, Carpentier A, Carandina G, Mazzoni E, Tognon M, et al. Age-specific seroprevalences of merkel cell polyomavirus, human polyomaviruses 6,7 , and 9 , and trichodysplasia spinulosa-associated polyomavirus. Clin Vaccine Immunol. 2013;20(3):363-8.

49. van der Meijden E, Bialasiewicz S, Rockett RJ, Tozer SJ, Sloots TP, Feltkamp MC. Different serologic behavior of MCPyV, TSPyV, HPyV6, HPyV7 and HPyV9 polyomaviruses found on the skin. PloS one. 2013;8(11):e81078.

50. Kamminga S, van der Meijden E, Wunderink HF, Touze A, Zaaijer HL, Feltkamp MCW. Development and Evaluation of a Broad Bead-Based Multiplex Immunoassay To Measure IgG Seroreactivity against Human Polyomaviruses. Journal of clinical microbiology. 2018;56(4). 
51. Madeleine MM, Carter JJ, Johnson LG, Wipf GC, Davis C, Berg D, et al. Risk of squamous cell skin cancer after organ transplant associated with antibodies to cutaneous papillomaviruses, polyomaviruses, and TMC6/8 (EVER1/2) variants. Cancer medicine. 2014;3(5):1440-7.

52. Malhotra J, Waterboer T, Pawlita M, Michel A, Cai Q, Zheng W, et al. Serum biomarkers of polyomavirus infection and risk of lung cancer in never smokers. British Journal of Cancer. 2016;115(9):1131-9.

53. Tyson GL, El-Serag HB. Risk factors for cholangiocarcinoma. Hepatology. 2011;54(1):173-84.

54. Gores GJ. Cholangiocarcinoma: current concepts and insights. Hepatology. 2003;37(5):961-9.

55. Burak K, Angulo P, Pasha TM, Egan K, Petz J, Lindor KD. Incidence and risk factors for cholangiocarcinoma in primary sclerosing cholangitis. The American journal of gastroenterology. 2004;99(3):523-6.

56. Chan JF, Tee KM, Choi GK, Zhu Z, Poon RW, Ng KT, et al. First detection and complete genome sequence of a phylogenetically distinct human polyomavirus 6 highly prevalent in human bile samples. J Infect. 2017;74(1):50-9.

57. Klufah F, Mobaraki G, Chteinberg E, Alharbi RA, Winnepenninckx V, Speel EJM, et al. High Prevalence of Human Polyomavirus 7 in Cholangiocarcinomas and Adjacent Peritumoral Hepatocytes: Preliminary Findings. Microorganisms. 2020;8(8).

58. Gardner SD, Field AM, Coleman DV, Hulme B. New human papovavirus (B.K.) isolated from urine after renal transplantation. Lancet. 1971;1(7712):1253-7.

59. Heritage J, Chesters PM, McCance DJ. The persistence of papovavirus BK DNA sequences in normal human renal tissue. Journal of medical virology. 1981;8(2):143-50.

60. Galed-Placed I, Valbuena-Ruvira L. Decoy cells and malignant cells coexisting in the urine from a transplant recipient with BK virus nephropathy and bladder adenocarcinoma. Diagnostic cytopathology. 2011;39(12):933-7.

61. Zhang Y, Ahmed H, Haririan A, Ugarte R, Papadimitriou JC, Drachenberg CB. Granulomatous inflammation in BK polyomavirus-associated nephropathy. Transpl Infect Dis. 2018;20(5):e12939.

62. Roberts IS, Besarani D, Mason P, Turner G, Friend PJ, Newton R. Polyoma virus infection and urothelial carcinoma of the bladder following renal transplantation. British journal of cancer. 2008;99(9):1383-6.

63. Klufah F, Mobaraki G, Hausen AZ, Samarska IV. Reactivation of BK Polyomavirus in Urine Cytology Is Not Associated with Urothelial Cell Carcinoma. Viruses. 2020;12(12):1412.

64. Herawi M, Parwani AV, Chan T, Ali SZ, Epstein JI. Polyoma virus-associated cellular changes in the urine and bladder biopsy samples: a cytohistologic correlation. The American journal of surgical pathology. 2006;30(3):345-50.

65. Lu H, Elsheikh TM, Zhang Y. Polyomavirus (BK) cytopathic effect in urine cytology is not associated with high risk of developing high-grade urothelial carcinoma. Journal of the American Society of Cytopathology. 2020;9(2):84-8.

66. Hirsch HH, Brennan DC, Drachenberg CB, Ginevri F, Gordon J, Limaye AP, et al. Polyomavirusassociated nephropathy in renal transplantation: interdisciplinary analyses and recommendations. Transplantation. 2005;79(10):1277-86. 
67. Starrett GJ, Buck CB. The case for BK polyomavirus as a cause of bladder cancer. Current opinion in virology. 2019;39:8-15.

68. Viarisio D, Müller-Decker K, Accardi R, Robitaille A, Dürst M, Beer K, et al. Beta HPV38 oncoproteins act with a hit-and-run mechanism in ultraviolet radiation-induced skin carcinogenesis in mice. PLoS pathogens. 2018;14(1):e1006783.

69. Skinner GR. Transformation of primary hamster embryo fibroblasts by type 2 simplex virus: evidence for a "hit and run" mechanism. Br J Exp Pathol. 1976;57(4):361-76.

70. Engels EA, Pfeiffer RM. Malignant thymoma in the United States: demographic patterns in incidence and associations with subsequent malignancies. International journal of cancer. 2003;105(4):546-51.

71. Sanjuan N, Porrás A, Otero J, Perazzo S. Expression of major capsid protein VP-1 in the absence of viral particles in thymomas induced by murine polyomavirus. Journal of virology. 2001;75(6):2891-9.

72. Rennspiess D, Pujari S, Keijzers M, Abdul-Hamid MA, Hochstenbag M, Dingemans AM, et al. Detection of human polyomavirus 7 in human thymic epithelial tumors. J Thorac Oncol. 2015;10(2):360-6.

73. Chteinberg E, Klufah F, Rennspiess D, Mannheims MF, Abdul-Hamid MA, Losen M, et al. Low prevalence of Merkel cell polyomavirus in human epithelial thymic tumors. Thorac Cancer. 2019;10(3):445-51.

74. Harms PW, Harms KL, Moore PS, DeCaprio JA, Nghiem P, Wong MKK, et al. The biology and treatment of Merkel cell carcinoma: current understanding and research priorities. Nature reviews Clinical oncology. 2018;15(12):763-76.

75. Hamiter M, Asarkar A, Rogers D, Moore-Medlin T, McClure G, Ma X, et al. A pilot study of Merkel cell polyomavirus in squamous cell carcinoma of the tongue. Oral oncology. 2017;74:111-4.

76. Salakova M, Koslabova E, Vojtechova Z, Tachezy R, Sroller V. Detection of human polyomaviruses MCPyV, HPyV6, and HPyV7 in malignant and non-malignant tonsillar tissues. Journal of medical virology. 2016;88(4):695-702.

77. Kantola K, Sadeghi M, Lahtinen A, Koskenvuo M, Aaltonen LM, Mottonen M, et al. Merkel cell polyomavirus DNA in tumor-free tonsillar tissues and upper respiratory tract samples: implications for respiratory transmission and latency. Journal of clinical virology : the official publication of the Pan American Society for Clinical Virology. 2009;45(4):292-5.

78. Marur S, D'Souza G, Westra WH, Forastiere AA. HPV-associated head and neck cancer: a virus-related cancer epidemic. The Lancet Oncology. 2010;11(8):781-9.

79. Laco J, Vosmikova H, Novakova V, Celakovsky P, Dolezalova H, Tucek L, et al. The role of high-risk human papillomavirus infection in oral and oropharyngeal squamous cell carcinoma in non-smoking and non-drinking patients: a clinicopathological and molecular study of 46 cases. Virchows Archiv : an international journal of pathology. 2011;458(2):179-87.

80. Mulder FJ, Pierssens D, Baijens LWJ, Kremer B, Speel EM. Evidence for different molecular parameters in head and neck squamous cell carcinoma of nonsmokers and nondrinkers: Systematic review and meta-analysis on HPV, p16, and TP53. Head \& neck. 2021;43(1):303-22.

81. Farshadpour F, Hordijk GJ, Koole R, Slootweg PJ. Head and neck squamous cell carcinoma in non-smoking and non-drinking patients with multiple tumors: etiologic significance of p53 and 
Ki-67 in non-tumorous epithelium. Journal of oral pathology \& medicine : official publication of the International Association of Oral Pathologists and the American Academy of Oral Pathology. 2008;37(9):549-54.

82. Mulder FJ, Klufah F, Janssen FME, Farshadpour F, Willems SM, De Bree R, et al. Presence of Human Papillomavirus and Epstein-Barr Virus, but Absence of Merkel Cell Polyomavirus, in Head and Neck Cancer of Non-Smokers and Non-Drinkers. Frontiers in oncology. 2021;10.

83. Babakir-Mina M, Ciccozzi M, Bonifacio D, Bergallo M, Costa C, Cavallo R, et al. Identification of the novel KI and WU polyomaviruses in human tonsils. Journal of clinical virology : the official publication of the Pan American Society for Clinical Virology. 2009;46(1):75-9. 


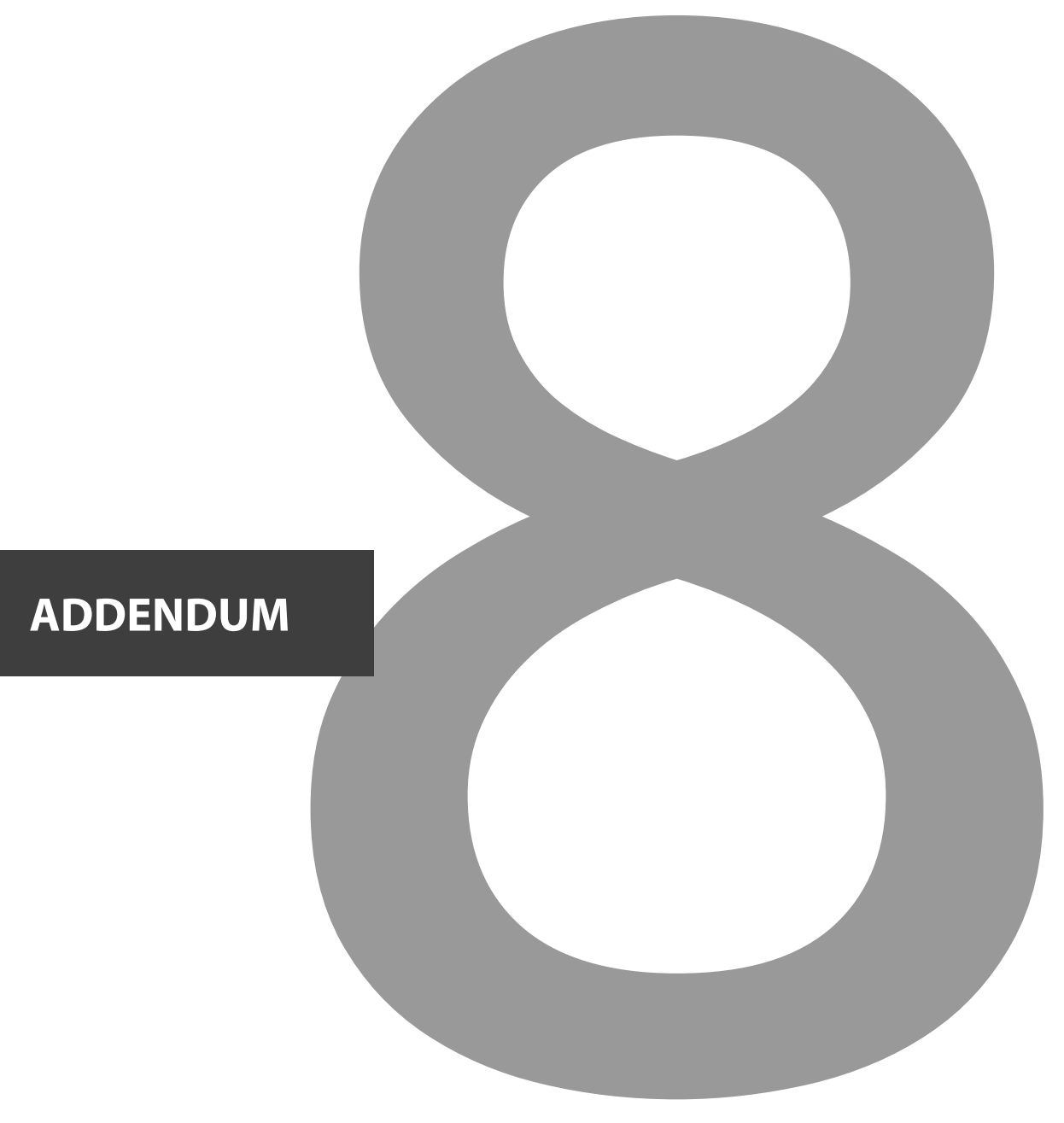


Summary 
Although the incidence of cancer is rapidly growing worldwide, the etiology of many malignant neoplasms remains largely unknown and thus also their cure. The link between the development of cancer and viral infections has been established since long. Globally, viruses have been demonstrated to be the causative agents of up to $15 \%$ of all human malignancies. During the recent two decades, the invention of new molecular techniques such as Next Generation Sequencing technology has marked a turning point by helping to discover new viruses, including novel members of human polyomaviruses (HPyVs). However, only very few HPyVs have been involved in neoplastic human diseases, which is mainly MCPyV, which was identified to be the major etiological agent of $80 \%$ of Merkel cell carcinomas (MCC). Much remains to be investigated concerning the putative role of HPyVs in human carcinogenesis. The main aim of the research described in this thesis was to screen and test for the presence of novel HPyVs in diverse human cancer tissues to get more insight into the role and possible involvement of these viruses in human tumorigenesis. For this purpose, we used FFPE tissues and applied a diverse sensitive, and specific set of molecular techniques to detect HPyV's at a single-cell level within the histomorphological context.

Chapter one is a general introduction summarizing the direct or indirect role of DNA and RNA tumor viruses in carcinogenesis. Furthermore, this chapter focused more on describing the polyomaviruses in general, then a brief background of each known HPyV. In addition, the outline and the aim of the research works in this thesis are explained. In chapter two, the current knowledge on the emerging role of the skin-associated HPyV6 and 7 in human cancers was comprehensively reviewed. Notably, previous studies revealed that the seropositivity of $\mathrm{HPyV} 6$ was found to be higher than $\mathrm{HPyV} 7$ and increased with age. Although an interesting finding, $\mathrm{HPyV} 6$ and 7 prevalence were higher in malignancy tissues than in non-neoplastic tissues. Furthermore, HPyV6 was detected more frequently in skin malignancies while $\mathrm{HPyV7}$ was found to be more frequent in other cancers such as thymoma and cholangiocarcinoma. Based on the reviewed studies, we concluded that $\mathrm{HPyV} 6$ and 7 remain important putative candidates possibly contributing to the etiopathogenesis of human disease especially skin tumors.

In chapter three, the presence of novel HPyVs in cholangiocarcinomas (CCA) was assessed to evaluate their possible contribution to the etiology of this human malignancy. Only recently, the presence of HPyV6 DNA (27\%) has been reported by a Chinese research group in the bile fluid of CCA patients by PCR. Here, we aimed to investigate the prevalence of HPyVs in CCA tissues to elucidate possible clinicopathological correlations between HPyVs and CCA. Interestingly, HPyV7 (69\%) was highly prevalent in the CCA cohort, the next most frequent was MCPyV (24\%) followed by HPyV6 (14\%). An important finding of this study was that HPyV7, HPyV6, and MCPyV are hepatotropic viruses and able to infect non-neoplastic human hepatocytes, bile duct 
epithelium, and CCA tumor cells. Yet, it remains unclear if and how HPyVs might contribute to the tumorigenesis of CCA. An interpretation of these results could be that the frequent finding of HPyV's in the adjacent peritumoral hepatocytes might suggest a robust indirect role of these viruses by leading to chronic inflammation which ends up inducing CCA transformation.

$\mathrm{BKPyV}$ is well known to reactivate under immunosuppression, especially in the context of solid organ transplantations, e.g. kidney transplantations. BKPyV has been suspected to be a putative oncogenic virus in the development of urothelial cell carcinomas (UCC) in immunocompromised patients. In chapter four we assessed whether reactivation of $\mathrm{BKPyV}$ in Decoy-positive urine cytology specimens (UCS) predicts or plays a role in the development of UCC of the urinary bladder. We showed that BKPyV detection was not restricted to the urine samples of patients who were diagnosed with UCC of the urinary bladder but also detected in the urine of non-UCC patients. In contrast to the UCC tissue specimens, all tested FFPE tissues were BKPyV-negative by IHC and PCR. Therefore, $\mathrm{BKPyV}$ reactivation is not restricted to immunosuppression but also can be found in the $\mathrm{UCC}$ of the immunocompetent patients. Therefore, the role of $\mathrm{BKPyV}$ in the oncogenesis of the majority of urothelial cell carcinoma of the bladder is rather unlikely.

In chapter five we investigated the prevalence of $\mathrm{MCPyV}$ in human thymoma, which is a rare malignancy of unknown etiology. In animal models, Murine polyomavirus (MuPyV) had been reported to induce thymomas after injecting MuPyV capsid protein (VP1) in neonatal mice. In addition, a few years ago our research group demonstrated the high prevalence of $\mathrm{HPyV} 7$ in thymic epithelial tumors. However, we aimed to uncover if the recent tumor virus identified $\mathrm{MCPyV}$ is playing a role in the etiopathogenesis in human epithelial thymic tumors. The presence of MCPyV-DNA was detected in $19.4 \%$ of the cohort and the DNA, RNA, and protein of MCPyV were showed in the single-cell level of epithelial thymoma. The most likely conclusion for these findings is that $\mathrm{MCPyV}$ expression does not act the same mechanism as seen in MCC. Thus, the contribution of $\mathrm{MCPyV}$ to the etiopathogenesis of thymoma is unlikely at least based on our dataset which revealed less expression of viral DNA, RNA, and proteins in tumor cells.

In chapter six, tissues obtained from 119 patients with head and neck squamous cell carcinoma (HNSCC) who had no history of smoking tobacco or drinking alcohol were investigated for the presence of human papillomavirus (HPV), Epstein-Barr virus (EBV), and MCPyV. HPV and EBV have previously been shown to be important etiological factors in the development of HNSCC. Our data confirmed the presence of HPV and EBV in patients with HNSCC. In contrast, MCPyV-LTAg expression was not detected by both RNA-ISH and IHC. This is in contrast with previous studies which reported that MCPyV-DNA was detected in tongue SCC. The most likely explanation for this 
overt contradiction is simply explained by the fact that we assessed the presence of MCPyV-LTAg expression on the single-cell level using RNA-ISH and IHC while the other studies tested their cohort by DNA PCR only. Our findings strongly support that there is no etiological role for MCPyV in HNSCC tumorigenesis. In chapter seven, a general discussion of the major findings of this thesis is presented. 


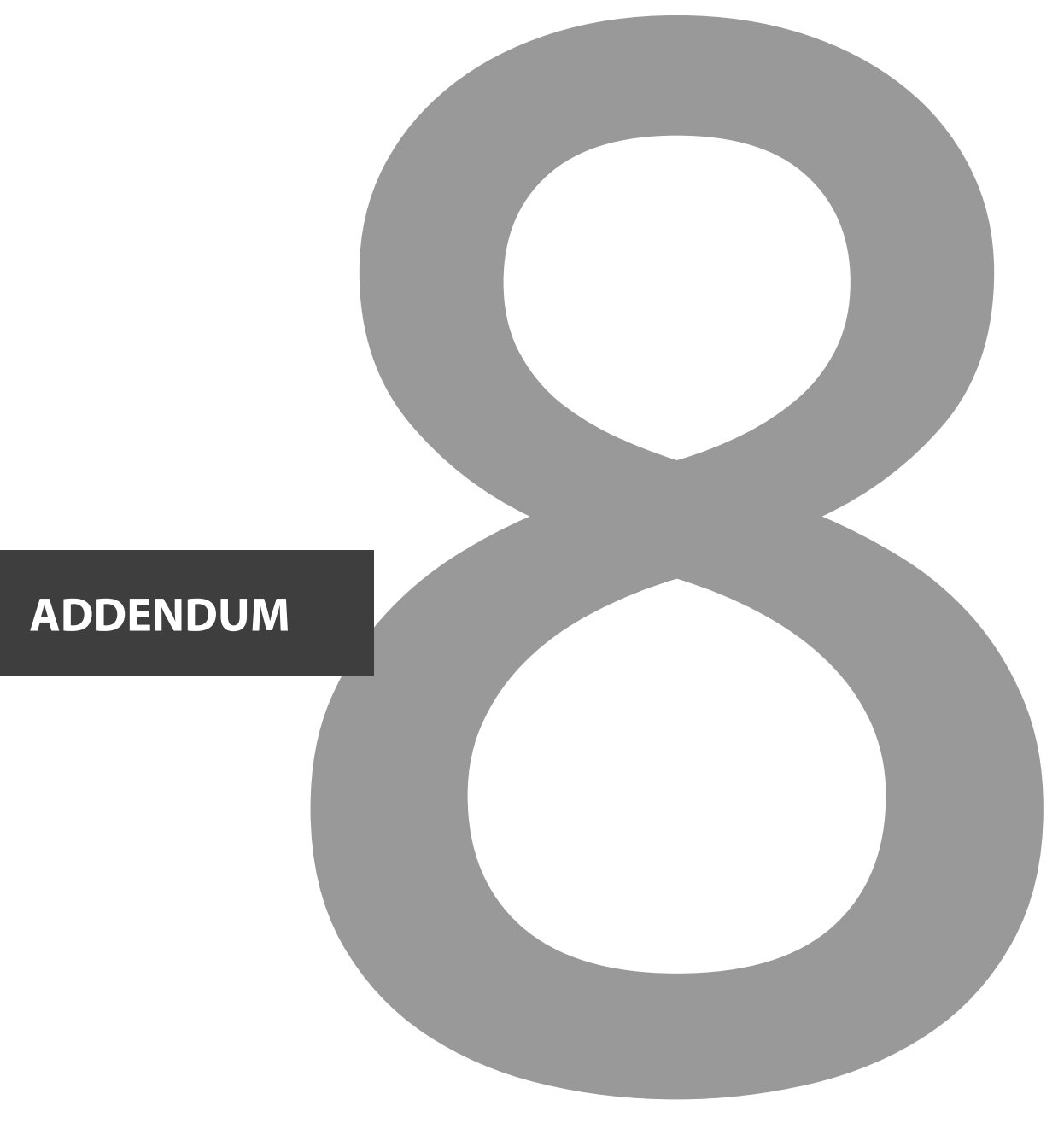


Impact 


\section{Social relevance}

Cancer is a leading global cause of death, accounting for nearly 9.6 million deaths worldwide in $2018{ }^{1}$. The global burden of cancer is rapidly rising because of population aging and low socioeconomic communities ${ }^{1,2}$. To date, the causative agent of many cancers remains unknown. Therefore, unraveling the underlying causes of diverse human cancers is crucial to mitigate the cancer burden by protecting the population from exposure to the respective carcinogens or at least limiting it by promoting novel prevention and therapeutic approaches.

Among all human cancers, approximately $10-15 \%$ of cancers are attributed to viral infection agents ${ }^{1,3-5}$. Shedding light on the detection of novel human tumor viruses is an important opportunity for cancer research by promoting novel prevention, and as a potential tool, early detection of these viruses might contribute to preventing early-stage pre-malignant lesions and thus progression to cancer. Importantly, some of the known human cancers induced by viruses can be prevented by vaccination 1 , 6,7. Human papillomavirus (HPV) which is directly linked to the etiopathogenesis of cervical carcinoma is a successful example from the past. The incidence of cervical cancer has already markedly declined in countries implementing the HPV vaccinations ${ }^{1,6,8}$. Additionally, the finding of HPV as an etiological factor of cervical carcinoma opened the gate to uncover the association of HPV with several other neoplasms including vagina, vulva, head, neck, anal, and penile carcinomas ${ }^{1,2}$. Therefore, there is a global call to all researchers to elucidate if currently known viruses or still to be discovered ones are implicated in human tumorigenesis. There is a continuous ongoing scientific debate regarding the role of human polyomaviruses (HPyVs) in human tumorigenesis. Here we focused to explore and providing more information on the role of HPyVs in human malignancies.

\section{Target group}

This dissertation aims to broaden and deepen our understanding of the presence and the possible role of HPyVs in human tumorigenesis. The HPyVs genome organization shares many similarities with the HPVs genome. Based on this it has been speculated that HPyVs might be involved in human tumorigenesis analogous to HPV 9-11. Our datasets could be of interest to many researchers, especially in the field of viral-related tumorigenesis. Additionally, we hope the outcome of this work will serve as a beneficial reference for researchers and professionals in viruses related to cancers in the future 12-16. Yet, the presence of HPyVs defined in this thesis is insufficient to be translated into preclinical or clinical practice until further confirmation of their role in the tumorigenesis. The recommendation of this thesis is that HPyV's remain candidates for human tumorigenesis, but more effort is needed to develop more advanced molecular techniques to elucidate the role of HPyV's in cancer. 


\section{Scientific impact}

The promising results obtained from the studies in this thesis have added to scientific knowledge since all the outcomes were published in a peer-reviewed journal as an openaccess manuscript to make them freely accessible online and presented at international and national congresses ${ }^{12-16}$. Additionally, the results of this thesis possibly provide the basis for relevant follow-up studies on HPyV's and human tumorigenesis.

The oncogenic potential role of the novel HPyVs in human tumorigenesis has not yet been completely uncovered except for MCPyV, which is the causative agent of $80 \%$ of MCC ${ }^{17-20}$. It is important to note that MCPyV-positive MCC appears to have better clinical outcomes and drug responses and a favorable survival rate compared to $\mathrm{MCPyV}$-negative MCC ${ }^{21-25}$. Given the potential implications of HPyV's in tumorigenesis, much more needs to be done to broaden the understanding of the involvement of these viruses in human carcinogenesis. To meet the immediate needs of elaborating the role of HPyV's, we aimed to provide insight into the presence of these viruses in various human malignancies and assess their association with tumors using a broad spectrum of molecular techniques.

HPyV6 and 7 contributions to human tumorigenesis are not yet elucidated. Studies about HPyV6 and 7 which shown a high seroprevalence in the human population and the ability to infect newborns ${ }^{13}$. Therefore, HPyV6 and 7 remain putative oncogenic viruses in humans for e.g. skin tumors as we discussed in detail in chapter 2. Moreover, a lot remains to be elucidated about HPyV6 and 7 such as their oncogenic potential, replication, latency, and tropism. This information will eventually help to understand the possible role of $\mathrm{HPyV} 6$ and 7 in carcinogenesis. As a consequence, these findings will be of major interest to stimulate further studies by researchers to bring advances in this field.

The etiology of Cholangiocarcinoma (CCA) is still largely unknown HPyV7, HPyV6, and MCPyV are reported for the first time as hepatotropic viruses and infecting bile duct epithelium and hepatocytes as shown in chapter $3^{14}$. The contribution of HPyV's to CCA carcinogenesis has not been definitely elucidated, but it is speculated that HPyV7 might act through an indirect mechanism to induce (chronic) inflammation as has been convincingly shown for Hepatitis B and C viruses in leading to hepatocellular carcinoma. In addition, the results and knowledge obtained from the study in chapter 3 will pave the way to study the possible role of HPyV's in the etiopathogenesis of other hepatobiliary diseases.

Early reports showed that that $\mathrm{BK}$ polyomavirus (BKPyV) is highly oncogenic in animal models ${ }^{30}$. This raised the question of whether BK polyomavirus (BKPyV) is a potential oncogenic virus in the development of urothelial cell carcinomas (UCC) in the urinary bladder ${ }^{31}$. In 2012, the International Agency for Research on Cancer 
(IARC) classified BKPyV as a group 2B possibly carcinogenic candidate to humans ${ }^{32}$. that the data in chapter $\mathbf{4}$ described that the reactivation of BKPyV detected in urine cytology is not associated with UCC ${ }^{15}$. In addition, BKPyV-reactivation is not restricted to immunosuppression but is also found in the urine of immunocompetent patients. Therefore, those findings open a new direction for the research which is that the intravesical treatment of UCC is possibly causing $\mathrm{BKPyV}$ reactivation, there is a need to perform further research to elucidate this relationship.

Thymoma is a rare tumor of unknown etiology. Previously, Murine polyomavirus was shown to induce thymomas in an animal model ${ }^{33}$. In addition, a high prevalence of $\mathrm{HPyV} 7$ in thymic epithelial tumors was reported ${ }^{34}$. MCPyV is closely linked to the etiopathogenesis of MCC and yet the only human carcinogenic polyomavirus ${ }^{20,35,36}$. Therefore, screening if $\mathrm{MCPyV}$ is involved in other human tumors is highly needed such as if $\mathrm{MCPyV}$ is playing a role in the etiopathogenesis in human thymoma ${ }^{16}$. The contribution of $\mathrm{MCPyV}$ to the etiopathogenesis of thymoma is unlikely as described in chapter 5. Altogether, HPyVs remain suspected as a putative oncogenic virus in the development of thymomas. Therefore, further research is needed to investigate other HPyV's in thymomas.

In 2018, about 453,000 deaths of head and neck squamous cell carcinoma (HNSCC) and 888,000 new cases were diagnosed ${ }^{1}$. Importantly, HPV is linked to the etiopathogenesis of HNSCC, particularly the oropharynx tumor. Given the potential role of involvement of HPV in HNSCC, other important factors correlated to the tumorigenesis such as aging, smoking, alcohol consumption, and gender ${ }^{1,2}$. A unique large cohort of nonsmokers and non-drinkers with HNSCC is investigated for the presence of three known human tumor viruses (HPV, EBV, and MCPyV) in chapter $6^{12}$. the results confirmed the presence of HPV and EBV in patients with HNSCC and no MCPyV was detected in this subset. This indicating that no etiological role of MCPyV in HNSCC tumorigenesis. Therefore, screening for other HPyVs in a cohort diagnosed with HNSCC without a history of smoking and alcohol drinking is needed,

Finally, the presence of HPyV's DNA and their association with human malignancies have commonly been tested by PCR alone which is insufficient to prove causality. Very few studies have shown the presence of these viruses on the single-cell level in the histomorphological context of the specific disease. In this thesis, advanced sensitive and specific molecular techniques were implemented such as FISH, RISH, and IHC to accurately determine the presence of HPyVs on a single cell level as we have shown in chapters 3 to 6. Even though, the molecular techniques we used (FISH, RISH, and IHC) for detecting the DNA, RNA, and protein of HPyVs are valuable assessment methods but remain insufficient to confirm the role of the respective virus in tumorigenesis. 
Implementing new powerful molecular technologies such as NGS to study the role of these viruses as potential oncogenic will seem to be a promising interesting avenue for further research. Therefore, there is an urgent need to apply additional molecular techniques to unravel the molecular basis of virus-host interactions and assess a possible role of viral integration or mutation of the LTAg in cancer development. 


\section{Reference:}

1. Wild CP WE, Stewart BW, editors. World Cancer Report: Cancer Research for Cancer Prevention. Lyon, France: International Agency for Research on Cancer Available from: http:// publicationsiarcfr/586 Licence: CC BY-NC-ND 30 IGO. 2020.

2. Bray F, Ferlay J, Soerjomataram I, Siegel RL, Torre LA, Jemal A. Global cancer statistics 2018: GLOBOCAN estimates of incidence and mortality worldwide for 36 cancers in 185 countries. CA: a cancer journal for clinicians. 2018;68(6):394-424.

3. De Martel C, Georges D, Bray F, Ferlay J, Clifford GM. Global burden of cancer attributable to infections in 2018: a worldwide incidence analysis. The Lancet Global Health. 2020;8(2):e180-e90.

4. Bouvard V, Baan R, Straif K, Grosse Y, Secretan B, El Ghissassi F, et al. A review of human carcinogens_-Part B: biological agents. The Lancet Oncology. 2009;10(4):321-2.

5. Plummer M, De Martel C, Vignat J, Ferlay J, Bray F, Franceschi S. Global burden of cancers attributable to infections in 2012: a synthetic analysis. The Lancet Global Health. 2016;4(9):e609-e16.

6. Bosch FX, Broker TR, Forman D, Moscicki AB, Gillison ML, Doorbar J, et al. Comprehensive control of human papillomavirus infections and related diseases. Vaccine. 2013;31 Suppl 7(Suppl 7):H1-31.

7. Chang MH. Cancer prevention by vaccination against hepatitis B. Recent results in cancer research Fortschritte der Krebsforschung Progres dans les recherches sur le cancer. 2009;181:85-94.

8. Dürst M, Gissmann L, Ikenberg H, zur Hausen H. A papillomavirus DNA from a cervical carcinoma and its prevalence in cancer biopsy samples from different geographic regions. Proceedings of the National Academy of Sciences of the United States of America. 1983;80(12):3812-5.

9. White EA, Kramer RE, Hwang JH, Pores Fernando AT, Naetar N, Hahn WC, et al. Papillomavirus E7 oncoproteins share functions with polyomavirus small $\mathrm{T}$ antigens. Journal of virology. 2015;89(5):2857-65.

10. Moens U. Human Polyomaviruses and Papillomaviruses. Int J Mol Sci. 2018;19(8).

11. DeCaprio JA, Garcea RL. A cornucopia of human polyomaviruses. Nat Rev Microbiol. 2013;11(4):26476.

12. Mulder FJ, Klufah F, Janssen FME, Farshadpour F, Willems SM, De Bree R, et al. Presence of Human Papillomavirus and Epstein-Barr Virus, but Absence of Merkel Cell Polyomavirus, in Head and Neck Cancer of Non-Smokers and Non-Drinkers. Frontiers in oncology. 2021;10.

13. Klufah F, Mobaraki G, Liu D, Alharbi RA, Kurz AK, Speel EJM, et al. Emerging role of human polyomaviruses 6 and 7 in human cancers. Infectious agents and cancer. 2021;16(1).

14. Klufah F, Mobaraki G, Chteinberg E, Alharbi RA, Winnepenninckx V, Speel EJM, et al. High Prevalence of Human Polyomavirus 7 in Cholangiocarcinomas and Adjacent Peritumoral Hepatocytes: Preliminary Findings. Microorganisms. 2020;8(8).

15. Klufah F, Mobaraki G, Hausen AZ, Samarska IV. Reactivation of BK Polyomavirus in Urine Cytology Is Not Associated with Urothelial Cell Carcinoma. Viruses. 2020;12(12):1412.

16. Chteinberg E, Klufah F, Rennspiess D, Mannheims MF, Abdul-Hamid MA, Losen M, et al. Low prevalence of Merkel cell polyomavirus in human epithelial thymic tumors. Thorac Cancer. 2019;10(3):445-51. 
17. Kuwamoto S. Recent advances in the biology of Merkel cell carcinoma. Human pathology. 2011;42(8):1063-77.

18. Fernandez-Figueras MT, Puig L, Musulen E, Gilaberte M, Lerma E, Serrano S, et al. Expression profiles associated with aggressive behavior in Merkel cell carcinoma. Modern pathology : an official journal of the United States and Canadian Academy of Pathology, Inc. 2007;20(1):90-101.

19. Shuda M, Feng H, Kwun HJ, Rosen ST, Gjoerup O, Moore PS, et al. T antigen mutations are a human tumor-specific signature for Merkel cell polyomavirus. Proceedings of the National Academy of Sciences of the United States of America. 2008;105(42):16272-7.

20. Feng H, Shuda M, Chang Y, Moore PS. Clonal integration of a polyomavirus in human Merkel cell carcinoma. Science (New York, NY). 2008;319(5866):1096-100.

21. Eid M, Nguyen J, Brownell I. Seeking Standards for the Detection of Merkel Cell Polyomavirus and its Clinical Significance. The Journal of investigative dermatology. 2017;137(4):797-9.

22. Moshiri AS, Doumani R, Yelistratova L, Blom A, Lachance K, Shinohara MM, et al. PolyomavirusNegative Merkel Cell Carcinoma: A More Aggressive Subtype Based on Analysis of 282 Cases Using Multimodal Tumor Virus Detection. The Journal of investigative dermatology. 2017;137(4):819-27.

23. Schrama D, Peitsch WK, Zapatka M, Kneitz H, Houben R, Eib S, et al. Merkel cell polyomavirus status is not associated with clinical course of Merkel cell carcinoma. The Journal of investigative dermatology. 2011;131(8):1631-8.

24. Harms KL, Zhao L, Johnson B, Wang X, Carskadon S, Palanisamy N, et al. Virus-positive Merkel Cell Carcinoma Is an Independent Prognostic Group with Distinct Predictive Biomarkers. Clinical cancer research : an official journal of the American Association for Cancer Research. 2021;27(9):2494-504.

25. Björn Andtback H, Björnhagen-Säfwenberg V, Shi H, Lui WO, Masucci GV, Villabona L. Sex Differences in Overall Survival and the Effect of Radiotherapy in Merkel Cell Carcinoma-A Retrospective Analysis of a Swedish Cohort. Cancers. 2021;13(2).

26. Blechacz B. Cholangiocarcinoma: Current Knowledge and New Developments. Gut Liver. 2017;11(1):13-26.

27. Tyson GL, El-Serag HB. Risk factors for cholangiocarcinoma. Hepatology. 2011;54(1):173-84.

28. Shaib YH, El-Serag HB, Davila JA, Morgan R, McGlynn KA. Risk factors of intrahepatic cholangiocarcinoma in the United States: a case-control study. Gastroenterology. 2005;128(3):620-6.

29. Burak K, Angulo P, Pasha TM, Egan K, Petz J, Lindor KD. Incidence and risk factors for cholangiocarcinoma in primary sclerosing cholangitis. The American journal of gastroenterology. 2004;99(3):523-6.

30. Levican J, Acevedo M, León O, Gaggero A, Aguayo F. Role of BK human polyomavirus in cancer. Infectious agents and cancer. 2018;13:12.

31. Roberts IS, Besarani D, Mason P, Turner G, Friend PJ, Newton R. Polyoma virus infection and urothelial carcinoma of the bladder following renal transplantation. British journal of cancer. 2008;99(9):1383-6.

32. Bouvard V, Baan RA, Grosse Y, Lauby-Secretan B, El Ghissassi F, Benbrahim-Tallaa L, et al. Carcinogenicity of malaria and of some polyomaviruses. The Lancet Oncology. 2012;13(4):339-40. 
33. Sanjuan N, Porrás A, Otero J, Perazzo S. Expression of major capsid protein VP-1 in the absence of viral particles in thymomas induced by murine polyomavirus. Journal of virology. 2001;75(6):2891-9.

34. Rennspiess D, Pujari S, Keijzers M, Abdul-Hamid MA, Hochstenbag M, Dingemans AM, et al. Detection of human polyomavirus 7 in human thymic epithelial tumors. J Thorac Oncol. 2015;10(2):360-6.

35. Wong SQ, Waldeck K, Vergara IA, Schroder J, Madore J, Wilmott JS, et al. UV-Associated Mutations Underlie the Etiology of MCV-Negative Merkel Cell Carcinomas. Cancer research. 2015;75(24):522834.

36. Toker C. Trabecular carcinoma of the skin. Archives of dermatology. 1972;105(1):107-10. 


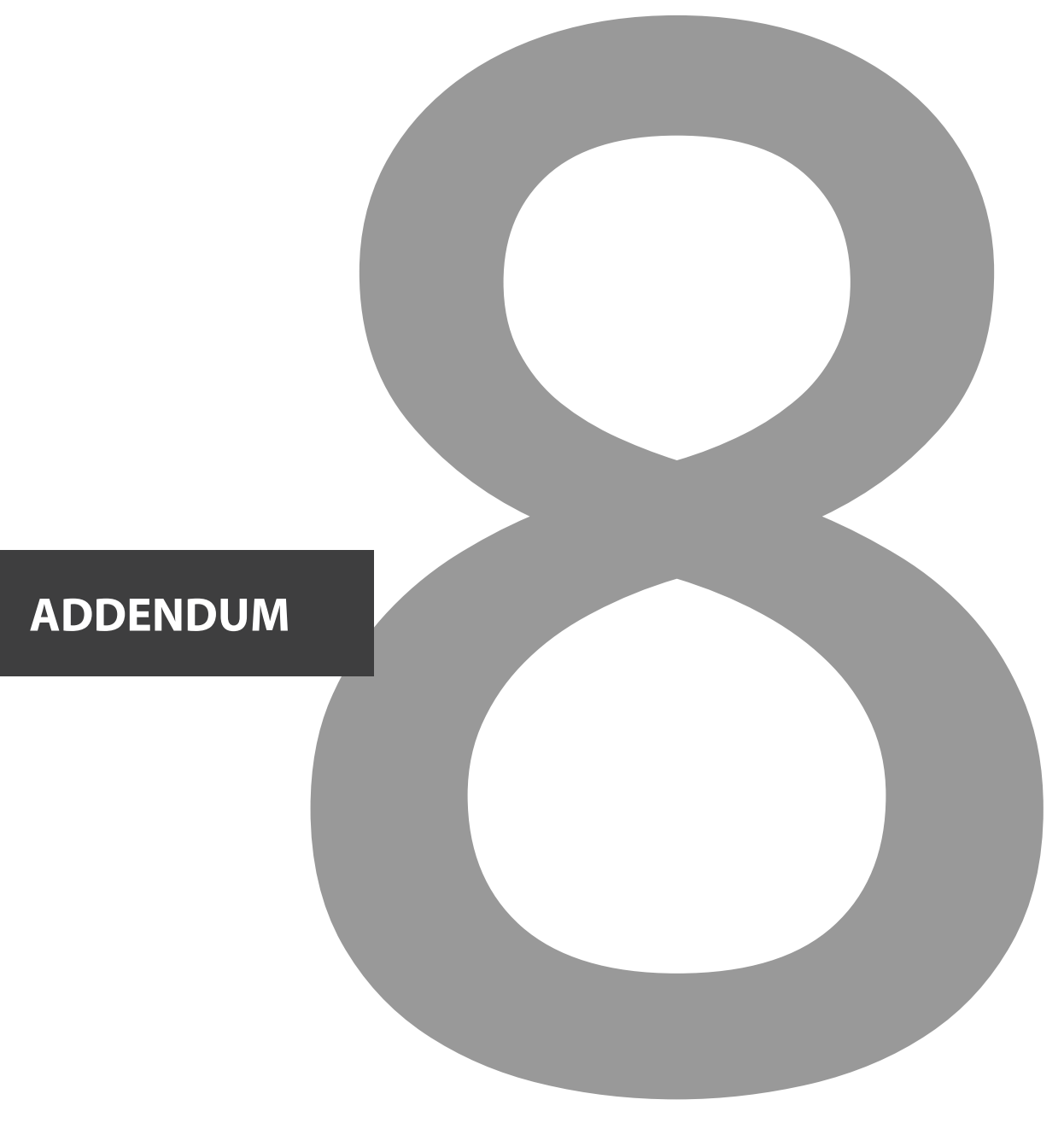


List of abbreviations 
AChR

AJCC

ALHE

ATL

BCC

BCG

BKPyV

bp

CCA

ChPyV

CLL

CMV

CTCL

DapB

dCCA

DFS

DNA

EBER

EBV

ELISA

FF

FFPE

FISH

FITC

GAPDH

GST

$\mathrm{HBV}$

$\mathrm{HCC}$

$\mathrm{HCV}$

HIV-1

HNSCC

$\mathrm{HPF}$

HPV

HPyV's

HPyV 6

HPyV 7

HPyV 9

HPyV 10

HPyV 12

Hs-PPIB
Anti-acetylcholine receptor

American Joint Committee on Cancer

Angiolymphoid hyperplasia with eosinophilia

Aggressive T-cell lymphoma

Basal cell carcinoma

bacillus Calmette-Guerin

BK polyomavirus

Base pair

Cholangiocarcinoma

Chimpanzee polyomavirus

Chronic lymphocytic leukemia

Cytomegalovirus

Cutaneous T-cell lymphoma

Dihydrodipicolinate reductase gene

Distal cholangiocarcinoma

Disease free survival

Deoxy ribonucleic acid

EBV encoded RNA

Epstein-Barr virus

Enzyme-linked immunosorbent assays

Fresh frozen tissue

Formalin fixed paraffin embedded

Fluorescence in situ hybridization

Fluorescein isothiocyanate

Glyceraldehyde-3-phosphate dehydrogenase

Glutathione-S-transferase

Hepatitis B virus

Hepatocellular carcinoma

Hepatitis C virus

Human immunodeficiency virus 1

Head and neck squamous cell carcinoma

High power field

Human papillomavirus

Human polyomaviruses

Human polyomavirus 6

Human polyomavirus 7

Human polyomavirus 9

Human polyomavirus 10

Human polyomavirus 12

Human peptidylprolyl isomerase B 
HTLV-1

IARC

ICC

iCCA

ICTV

IHC

ISH

JCPyV

KS

KIPyV

KS

KSHV

LIPyV

LTAg

MCC

$\mathrm{MCPyV}$

MG

MM

mRNA

$\mathrm{MuPyV}$

MWPyV

NGS

NJPyV

NPA

NPSCC

NSND

OPSCC

OS

OSCC

pCCA

PCR

PML

PNA

PP2A

pRB

PVAN

PyVNA

PyVs

QPyV
Human T-lymphotropic virus-1

International Agency on Research for Cancer

Immunocytochemistry

Intrahepatic cholangiocarcinoma

International Committee on Taxonomy of Viruses

Immunohistochemistry

In situ hybridization

JC polyomavirus

Kilobase

Karolinska institute polyomavirus

Kaposi sarcoma

Kaposi's sarcoma herpesvirus

Lyon IARC polyomavirus

large tumor antigen

Merkel cell carcinoma

Merkel cell polyomavirus

Myasthenia gravis

Malignant melanoma

Messenger ribonucleic acid

Murine polyomavirus

Malawi polyomavirus

Next generation sequencing

New jersey polyomavirus

Nasopharyngeal aspirates

Nasopharyngeal squamous cell carcinoma

Non-smokers and non-drinkers

Oropharyngeal squamous cell carcinoma

Overall survival

Oral squamous cell carcinoma

Perihilar cholangiocarcinoma

polymerase chain reaction

progressive multifocal leukoencephalopathy

Peptide nucleic acid

Protein phosphatase 2A

Retinoblastoma protein

Polyomavirus-associated nephropathy

BKPyV-associated nephropathy

Polyomaviruses

Quebec polyomavirus 
RCA

RISH

SCS

SSC

sTAg

StLPyV

SV40

$\mathrm{TAg}$

TMAs

TNM

TS

TSPyV

UCC

UCS

VLP

VP1

WHIM

WUPyV
Rolling circle amplification

RNA in situ hybridization

Specimen control size

Squamous cell carcinoma

Small tumor antigen

St. louis polyomavirus

Simian virus 40

Tumor antigen

Tissue microarray

Tumor-node-metastasis

Trichodysplasia spinulosa

Trichodysplasia spinulosa associated polyomavirus

Urothelial cell carcinoma

Urine cytology specimens

Virus-like particles

Viral protein 1

Warts, hypogammaglobulinemia, infections, and myelokathexis

Washington university polyomavirus 


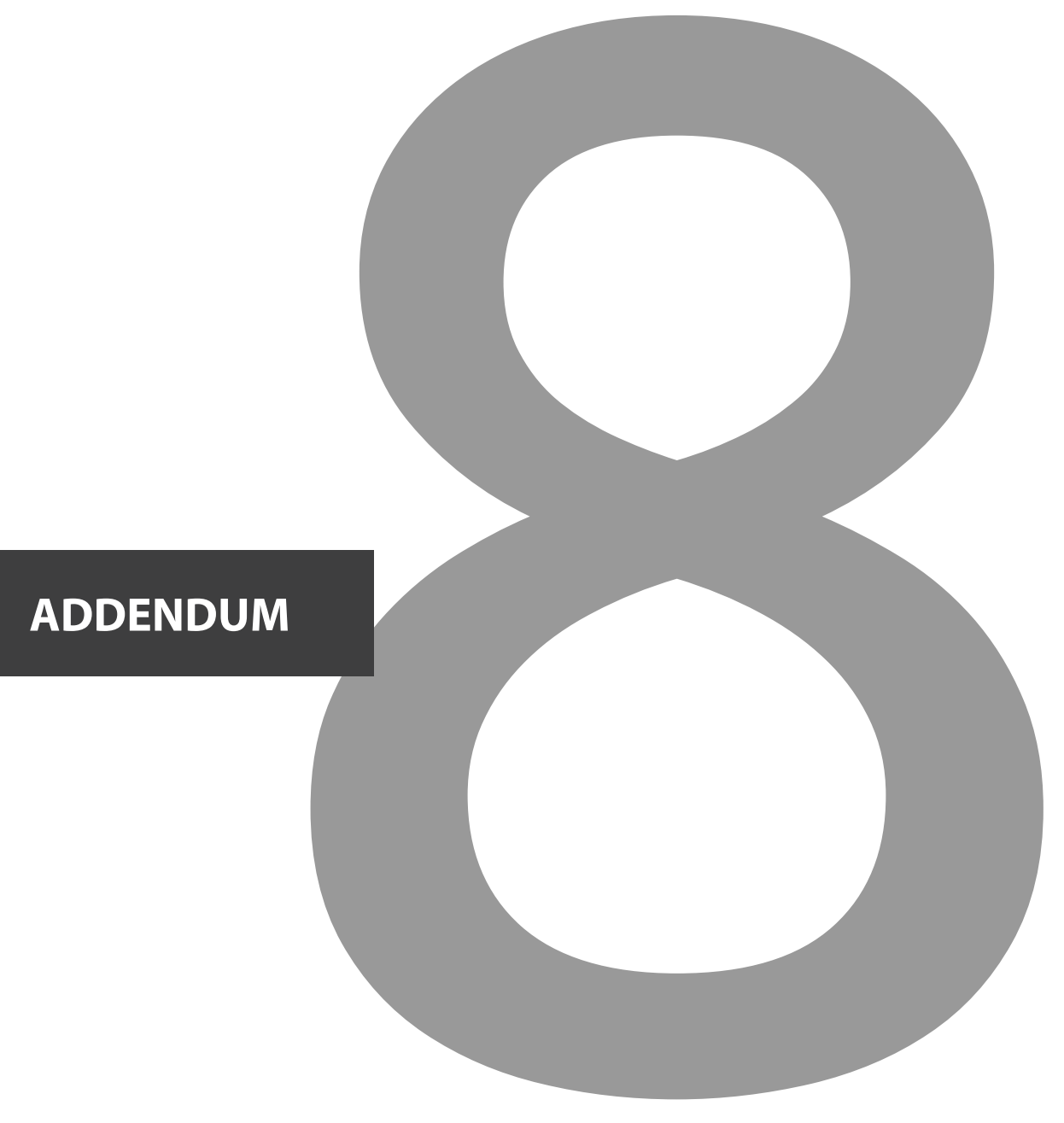


Acknowledgments 
Finally, my $\mathrm{PhD}$ journey has come to an end. It wouldn't be possible without all the wonderful people who surrounded and supported me, and I would like to take a moment to express my sincere gratitude to them.

First, I like to express my deep appreciation to my promotors. I have had the privilege of being guided by three experts, Prof. Dr. Axel zur Hausen, Prof. Dr. Ernst Jan M. Speel, and Dr. Véronique Winnepenninckx. Dear Axel, thank you for granting me the opportunity to work with you, for sharing your knowledge and experience. I am very grateful for your unwavering support, encouragement, and guidance during my PhD work. I count myself incredibly fortunate to have you as my supervisor. Every meeting with you was a crucial moment in my learning process in which I acquired a lot of knowledge and insights. Your confidence and consistent belief in me gave me the enthusiasm to move forward in my research and working with you lead me to strive for perfection down to the last detail. I appreciate all of your suggestions for improving my thesis work, and I thank you for proofreading it. Axel, you have been an incredible supervisor; I cannot thank you enough for everything you have done. I look forward to working with you on future projects.

Thank you, Ernst Jan, for the incredible support throughout these years, and for sharing your expertise and knowledge of molecular pathology. It was a great privilege and honor to work with you, and your suggestions and tips for the improvement of my work have been very helpful and instructive. Véronique, thank you for being helpful and supportive in many ways. Your facilitation of my work within the pathology department has been especially important. Thank you for your essential contributions to this thesis. I would like to thank the members of the assessment committee (Prof. Dr. M.G.J Tilanus, Prof. Dr. Ugo Lionel Moens, Prof. Dr. GA van Koeveringe, Dr. A.J.C van den Brule) for the time they spent on reading and assessing my thesis.

I also give my thanks to all the staff of the Department of Pathology, you have helped in different ways and have added a fascinating dimension throughout my thesis work. Thank you, Iryna Samarska, for all your help, which was critical for my thesis. You provided invaluable advice, support, and encouragement during my $\mathrm{PhD}$ journey. It has been a pleasure working with you and I hope we continue working together. Thank you, Dorit Rennspiess, for your support and knowledge, and despite being busy with your diagnostic work, you always found the time to help and support me. I greatly appreciate your expertise and your contribution to my projects. Many thanks to Prof. dr. Manon van Engeland, Benoit, Guido, Dr. Jan Beckervordersandforth, Dr. Lisa Hillen, Ronald Tonk, Andrea Ruland, Sabine for playing an important role in facilitating my work. Dear Maarten, thank you for being helpful whenever needed. 
Furthermore, I extend special thanks to the secretarial office staff. Thank you very much for the office manager Lysette, you helped me a lot from the first week of arriving in the Netherlands until to have my PhD studies finished, and you always solve any issue I faced. Thank you, Monique, for helping with my research materials and reagents orders and being helpful whenever needed. Many thanks to you, Audrey, Ingrid, Tonneke, and Danielle for your help and support. Thanks to my dearest past and present colleagues in all the pathology research groups (Laura, Jaleesa, Nikkie, Imke, Kim Lommen, Kim Smits, Selena, Nathalie, Simone, Amir, Frans, Emil, Lindsay, Glenn, Jenny, Kasper, Zheng, Renee, and Edith). I consider myself a very privileged person to have been surrounded by such supportive and friendly people.

I am incredibly blessed to have worked with the outstanding group of Axel's lab. Thank you, Ghalib, Dan, Shuai, Kim Severens, and Amanda, for your guidance and support, both personal and scientific, and for the beautiful memories we shared, and for keeping it casual and fun in the office. I wish you all the best with your research and hope to continue our collaboration. Thank you very much, Mat Rousch, for your technical assistance for me and everyone with many things in the research labs, you are genuinely passionate about your work and have a unique ability for sorting things out whenever there was an issue. Gregorio, thank you for all the discussions we had in the labs and for your invaluable help. Thank you Musa for your support, working with you on the pluripotent stem cell projects was a great pleasure and you made my work much easier with your expertise, I wish good luck with your research.

To all of the co-authors who contributed to the creation of the various articles presented in this thesis: I appreciate your extensive cooperation in getting our work published. To the bachelor's and master's student interns ( Diana, Mick, Yen Nhi, Martina, and Malte): Your thesis was inspired for me and my research. I was proud to be one of your project's supervisors. Thanks to my friend Dr. Abdullah Ibrahim for the support in proofreading my manuscripts and for the statistics help, your friendship means a lot to me which cannot be described in words.

Special thanks to the Saudi Embassy and Saudi Cultural Office in The Hague for support throughout my scholarship. I would like to thank my advisor Lieke Gieles for her guidance with kindness and for always being there to help. Also, I am extremely grateful to Al-Baha University for the scholarship and funding of my research projects. I would sincerely like to thank His Excellency Prof. Abdullah Yahya Alhussain the President of Albaha University and the Vice President for Graduate Studies and Scientific Research Prof. Ghanem Alghamdi for all the support during my PhD journey. I would also like to thank Dr. Shaia S. Almalki, Dr. Raed A. Alharbi, and Dr. Abdullah A. Alzahrani for their encouragement and guidance throughout my research. 
Finally, this thesis journey would not have been possible without my fantastic family's support, interest, and love. I give a heartfelt thank-you to my father, Mahdi, and my mother, Aisha there are not enough words to express how thankful I am to both of you. I am incredibly grateful for your continuous encouragement and support throughout my entire life. A special thank-you goes to my wife, Fatimah. I could not have accomplished any of this without your outstanding support, love, motivation, and encouragement during my education journey abroad. You have been a constant inspiration, and thank you for your endless amounts of love. Thank you, my darling, beautiful kids: Feras, Elias, Enas, and Almas, I am very lucky to have you in my life. A very warm thank-you also goes to my beloved brothers and sisters, Sultan, Ali, Taghreed, Yahya, Noura, Aayel, Mohammad, Sadeem, and Saja for your support and encouragement. Last but not least, many thanks to my uncle His Eminence Sheikh Ali Mongary and my aunt Saleha Jarallah, also for my cousins Ali Klufah and Walid Klufah for their supports and advice.

Eindelijk is het zover! Graag wil ik iedereen bedanken in The Netherlands die direct of indirect een bijdrage heeft geleverd aan mijn werk. 


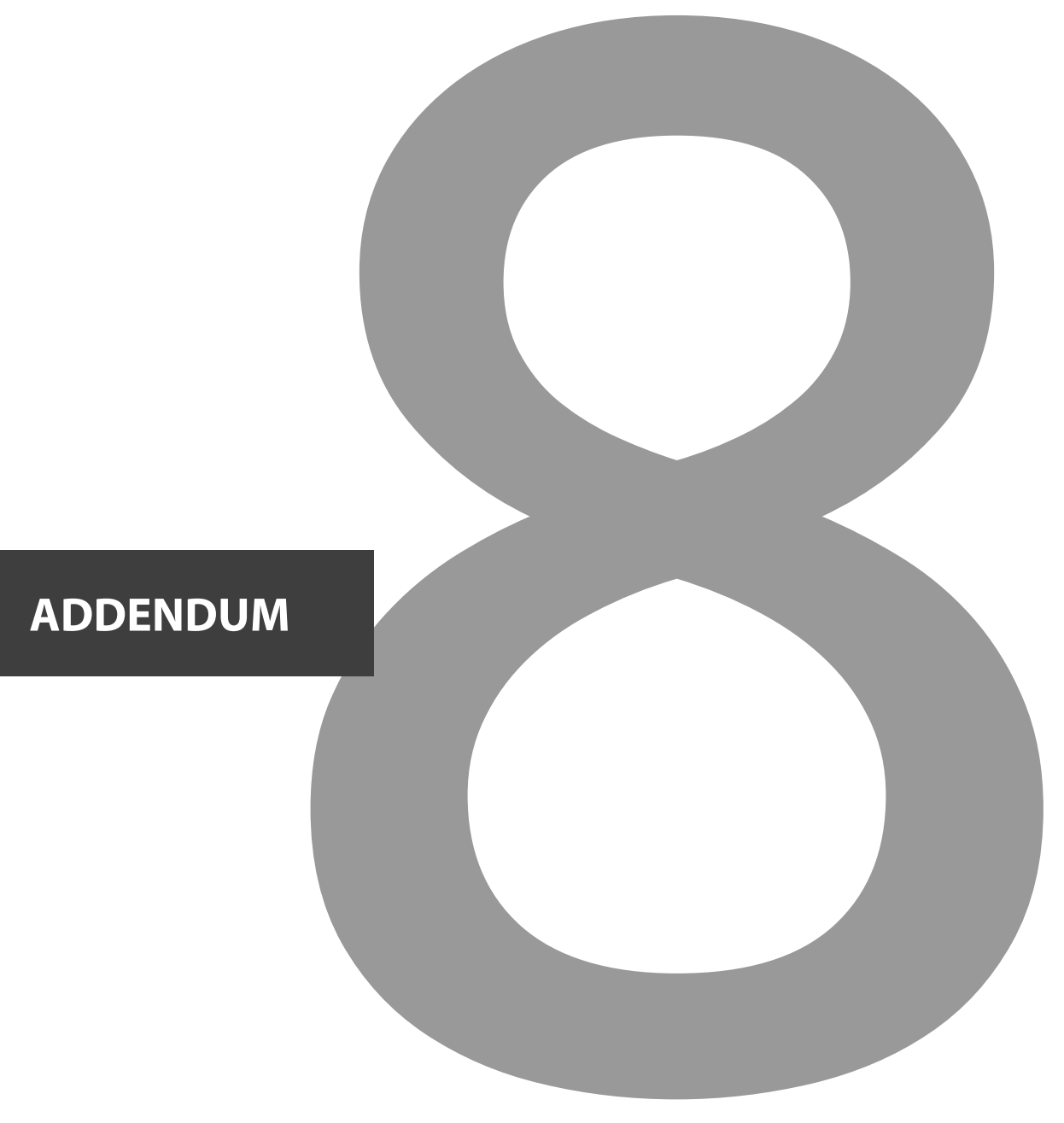


Curriculum Vitae 
Faisal Mahdi Klufah was born on October 26th, 1984, in Jazan, Saudi Arabia. His interest in medical laboratory sciences began after he completed his three-years associate degree in medical laboratory technology at King Khalid University in 2006. In the same year, he started working as a medical lab technician at Sharurah General Hospital, Sharurah, Saudi Arabia. In September 2007, he received a scholarship from the King Abdullah Scholarship Program to pursue his bachelor's and master's studies in the United States. In 2012, he obtained his bachelor's degree in clinical laboratory science at Northern Illinois University in DeKalb,

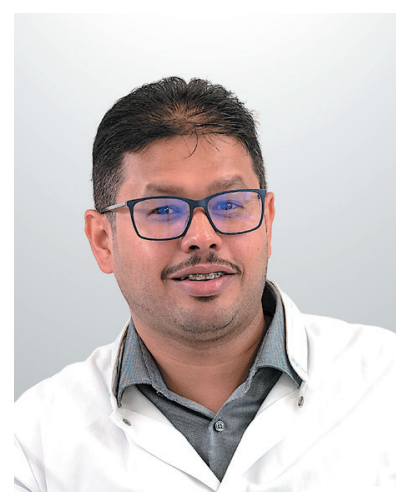
Illinois states. Also, he received the opportunity to perform a one-year clinical training as medical lab scientist at Rockford Memorial Hospital in Rockford, Illinois states. In June 2012, he passed the board exam of the American Society for Clinical Pathology (ASCP) and was certified as Medical Laboratory Scientist (MLS). In May 2014, he completed his master's degree in medical laboratory science with pathology subspecialty at Quinnipiac University in Hamden, Connecticut state. Afterward, he went back to Saudi Arabia and began working as a director of diagnostic laboratory services in Sharurah General Hospital. In December 2014, he moved to work as a lecturer at Albaha University in the department of laboratory medicine in the faculty of applied medical sciences besides working as a medical laboratory specialist at Albaha University Medical Center. In April 2017, he carried out his PhD trajectory in the Pathology Department, Maastricht University Medical Centre+ within the School for Oncology and Developmental Biology (GROW) at the faculty of Health, Medicine, and life sciences at Maastricht University. He worked under the supervision of prof. Dr. Axel zur Hausen, prof. Dr. Ernst Jan M. Speel, and Dr. Véronique Winnepenninckx for the project entitled "Towards Uncovering Polyomavirus-Carrying Human Cancers: Pathology and Implications for Tumorigenesis”. After finishing this $\mathrm{PhD}$ thesis, Faisal is going back to continue his work at Albaha University. 


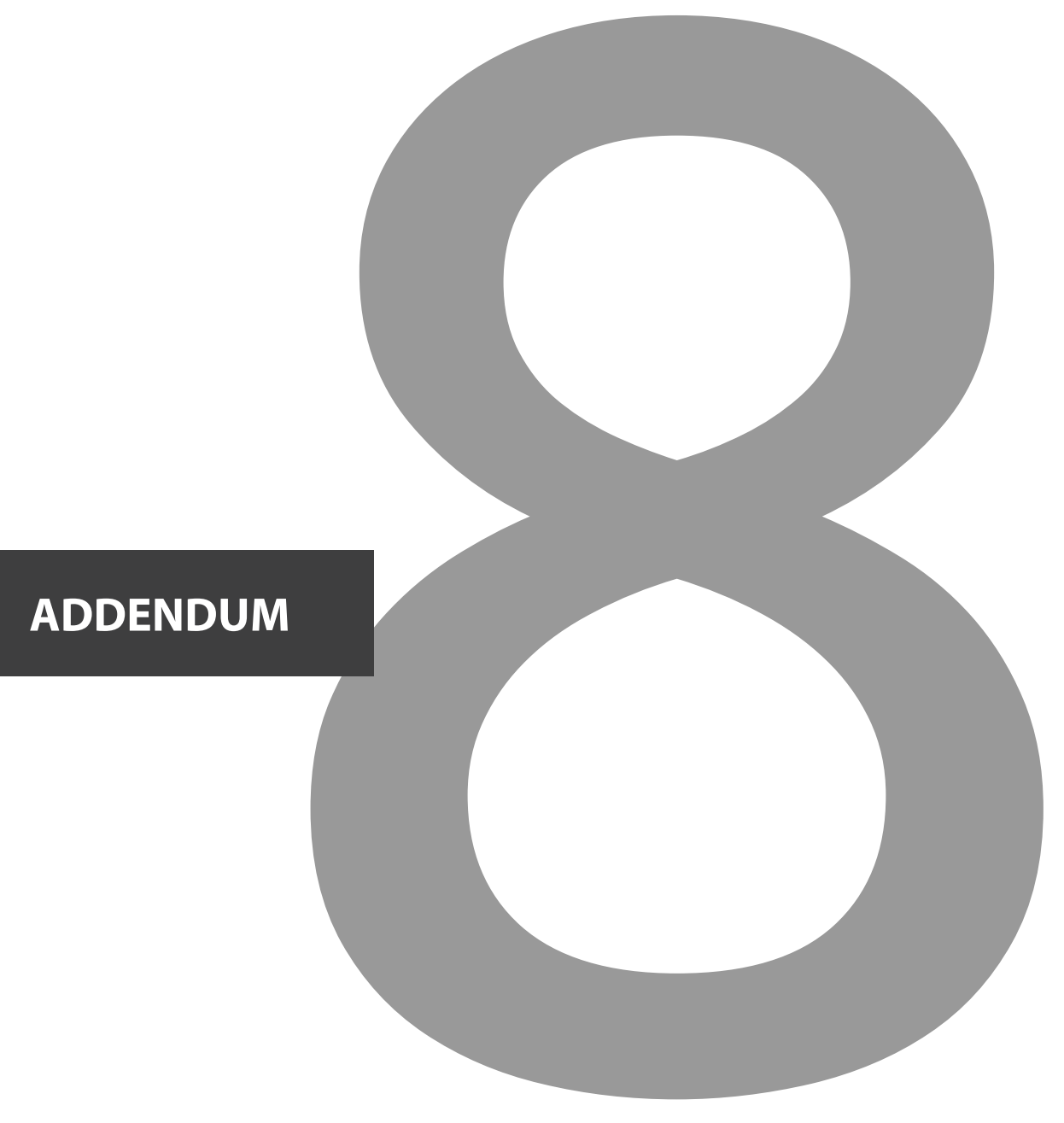




\section{List of publications and congresses participation}




\section{Publications:}

Klufah F, Mobaraki G, Chteinberg E, Alharbi RA, Winnepenninckx V, Speel EJM, et al. High Prevalence of Human Polyomavirus 7 in Cholangiocarcinomas and Adjacent Peritumoral Hepatocytes: Preliminary Findings. Microorganisms. 2020;8(8).

Klufah F, Mobaraki G, Hausen AZ, Samarska IV. Reactivation of BK Polyomavirus in Urine Cytology Is Not Associated with Urothelial Cell Carcinoma. Viruses. 2020;12(12):1412.

Klufah F, Mobaraki G, Liu D, Alharbi RA, Kurz AK, Speel EJM, et al. Emerging role of human polyomaviruses 6 and 7 in human cancers. Infectious agents and cancer. 2021;16(1).

Mulder FJ, Klufah F, Janssen FME, Farshadpour F, Willems SM, De Bree R, et al. Presence of Human Papillomavirus and Epstein-Barr Virus, but Absence of Merkel Cell Polyomavirus, in Head and Neck Cancer of Non-Smokers and Non-Drinkers. Frontiers in oncology. 2021;10.

Chteinberg E, Klufah F, Rennspiess D, Mannheims MF, Abdul-Hamid MA, Losen M, et al. Low prevalence of Merkel cell polyomavirus in human epithelial thymic tumors. Thorac Cancer. 2019;10(3):445-51.

\section{Oral presentations at scientific congresses:}

F. Klufah, E. Chteinberg, R. A. Alharbi, A. K. Kurz, V. Winnepenninckx, E.-J. Speel, A. zur Hausen

"Detection of human polyomavirus 6 and 7 in the human cholangiocarcinoma tissues" 103. Jahrestagung der Deutschen Gesellschaft für Pathologie (German Society for Pathology), Frankfurt Am Main, Germany, 2019 June 13-15

F. Klufah, G. Mobaraki, E. Chteinberg, R. A. Alharbi, A. K. Kurz, V. Winnepenninckx, E.-J. Speel, I. Samarska, A. zur Hausen

"Detection of Human Polyomavirus 6 and 7 in the Human Cholangiocarcinoma Tissues" ICGEB DNA Tumour Virus Meeting - 50th Anniversary, Trieste, Italy, 2019 July 9th14 th 


\section{Poster presentations at scientific congresses:}

F. Klufah, E. Chteinberg, G. Mobaraki, R.A. Alharbi, A.K. Kurz, V. Winnepenninckx, E. Speel, A. zur Hausen

"Detection of human polyomavirus 6 and 7 in the human cholangiocarcinoma tissues" 31 st European Congress of Pathology, Nice, France from 7-11 September 2019

F. Klufah, G. Mobaraki, E. Chteinberg, R.A. Alharbi, A.K. Kurz, V. Winnepenninckx, E. Speel, I. Samarska, A. zur Hausen

"Human Polyomaviruses 6 and 7 and Merkel Cell Polyomavirus are Hepatotropic viruses" The United States and Canadian Academy of Pathology (USCAP) 2020, Los Angeles Convention Center - Los Angeles, CA, February 28-March 5, 2020

F. Klufah, G. Mobaraki, A. zur Hausen, I. Samarska

"Detection of Human Polyomavirus JCPyV DNA in Urothelial Carcinomas of Urinary Bladder"

33rd European Congress of Pathology, 29-31 August 2021

I. Samarska, F. Klufah, G. Mobaraki, A. zur Hausen

"Relation of BK virus-positive urinary cytology to urothelial cell carcinoma of the urinary bladder"

The United States and Canadian Academy of Pathology (USCAP) 2020, Los Angeles Convention Center - Los Angeles, CA, February 28-March 5, 2020

F. J. Mulder, F. Klufah, F. M. E. Janssen, F. Farshadpour, S. M. Willems, R. de Bree, A. zur Hausen, M. F. C. M. van den Hout, B. Kremer, and E. M. Speel 2 "The usual viral suspects in head and neck cancer of non-smokers and non-drinkers: an analysis of HPV, EBV, and MCPyV"

7th WORLD CONGRESS of the International Academy of Oral Oncology, IAOO 2019, ROME Italy 31 August to 3 September 


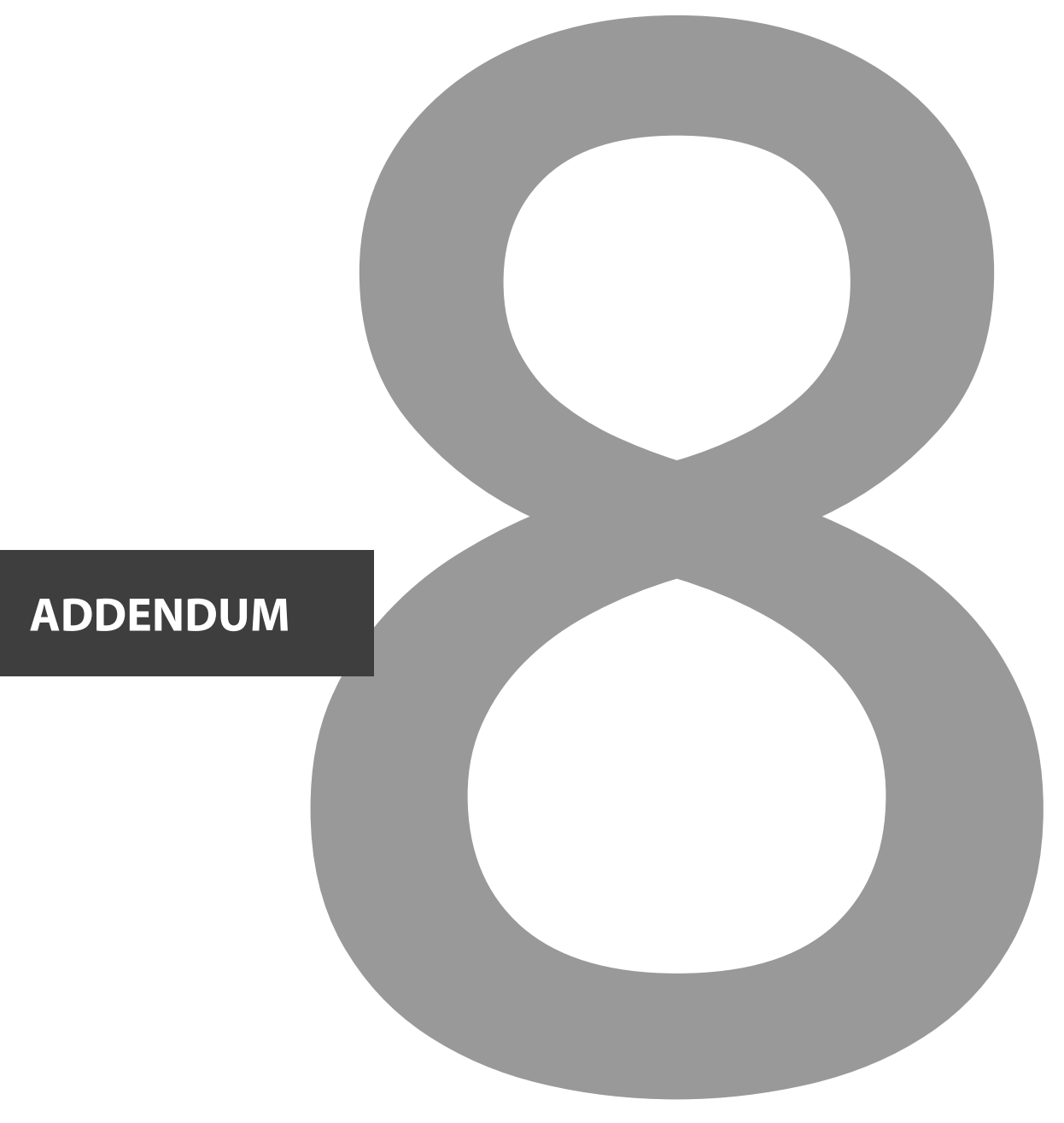


Summary in Arabic 
و اللوزتين، إلا أنه لم يثبت وجود علاقة سببية بين وجود هذا الفيروس في تكوّن هذه السرطانات إلى الآن.

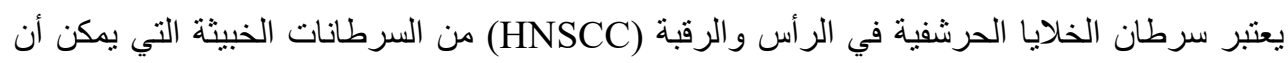

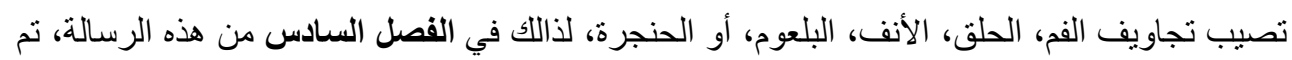

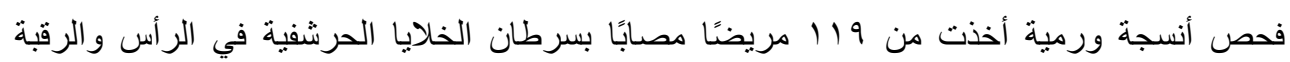

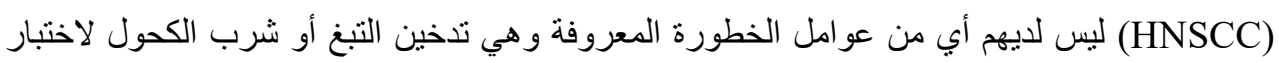

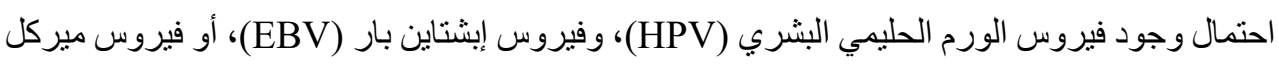

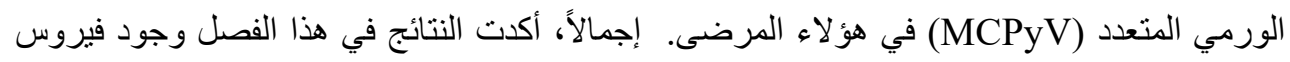

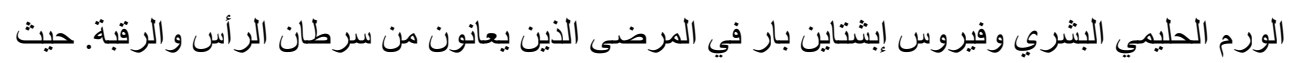

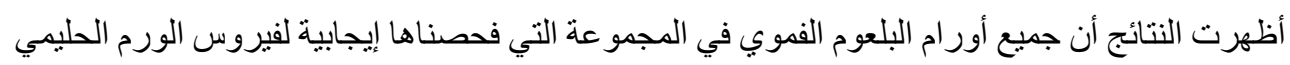

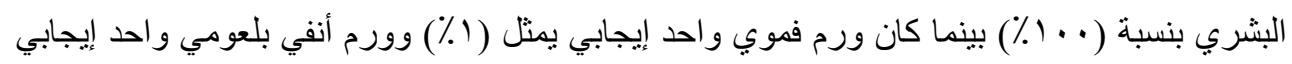
ويمثل (0 \%) من المجمو عة البحثية، بالإضافة إلى ذلك كانت ثلاث عينات لأورام الأنف البلعومي إيجابية

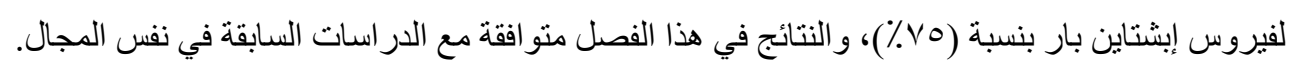

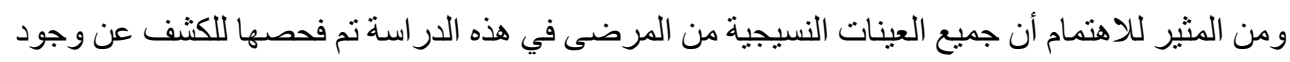

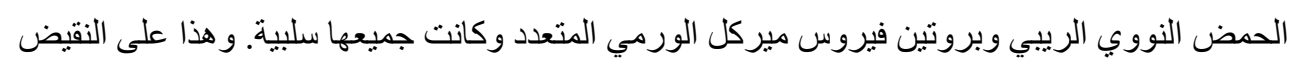

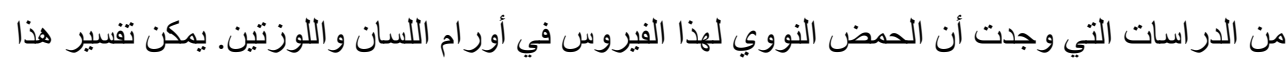
التناقض بأن الدر اسات السابقة استخدمت فحص تفاعل البلمرة المتسلسل (PCR) و هو اختبار لا يمكن منه فئه

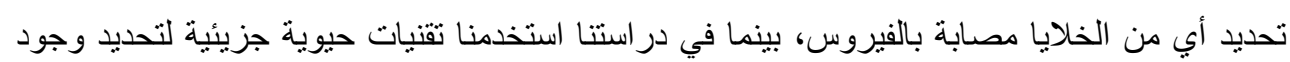

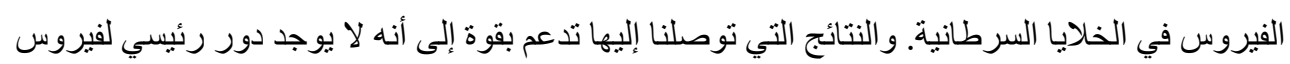
ميركل الورمي المتعدد (MCPyV) في تكوّن أورام الر أس والرقبة.

أخيرًا في الفصل السابع من هذه الرسالة، قمت بعرض مناقثة عامة لأهم النتائج التي توصلت إليها هذه 
عينات بول مرضى سرطان المثانة البولية، بل تم اكتثاف الفيروس أيضًا في عينات بول مرضى يعانون من أمر اض غير سرطانية، على سبيل المثال مرضى زر اعة الكلى أو الأمر اض المزمنة التي تتطلب تثبيط المناعة. على النقيض من عينات البول، بينت النتائج في هذا الفصل أن جميع عينات الأنسجة السرطانية الموجودة في ورم المثانة البولية سلبية للفيروس للمرضى المصابين بسرطان المثانة البولية باستخدام فحص تفاعل البلمرة المتسلسل (PCR) و الكيمياء النسيجية المناعية (IHC). علاوة على لذلك، نتائج هذا الفصل تشير إلى أن إعادة تنشيط الفيروس الورمي المتعدد الأول (BKPyV) لا يقتصر على تثبيط المناعة فحسب، بل يمكن أن يعيد الفيروس نشاطه في مرضى سرطان المثانة للمرضى غير المثبطين مناعياً. لللك خلص هذا الفصل إلى أنه من الأرجح لا يوجد دور للفيروس الورمي المتعدد الأول (BKPyV) في تكوّن الأورام لغالبية سرطان خلايا الظهارة البولية في المثانة.

من الأورام التي ماز ال سبب تكونها مجهول هو ورم الغدة الصعترية (Thymoma)، و الذي بطلق عليه ورم التوتة، يعتبر من الأورام النادرة التي تحدث في الغالب عند الأشخاص اللذين تجاوزوا الخمسين عاماً. من الدر اسات التي قام بها الباحثون لمعرفة إن كان للفيروسات دور في هذا الورم، هي دارسة أول فيروس

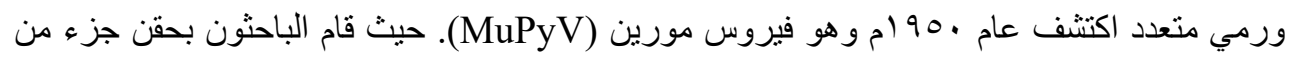
بروتين فيروس مورين في فئران حديثة الولادة فوجدوا أنها سببت ورم الغدة الصعترية في الفئران. بالإضافة إلى ذلك، أظهر فريقنا البحثي قبل بضع سنوات عن وجود الفيروس الورمي المتعدد السابع بنسبة عالية في أورام الغدة الصعترية. لذلك هدفنا في الفصل الخامس إلى عمل در اسة للكثف (HPyV7) عما إذا كان سرطان الغدة الصعترية (ورم التوتة) قد ينشأ بسبب فيروس ميركل الورمي المتعدد وهو فيروس معروف بأنه يسبب ورم جلدي خبيث. ومن خلال فحص الأنسة السرطانية للغدة الصعترية باستخدام تفاعل البلمرة المتسلسل (PCR) وجدت الدراسة أن فيروس ميركل الورمي المتعدد موجود بنسبة ؛،9 ( ٪. كما أظهرت البيانات أن وجود الحمض النووي، و الحمض النووي الريبي، وبروتين فيروس ميركل على مستوى الخلية أقل في الخلايا السرطانية لورم التوتة، وبالتالي؛ من غير المحتمل أن بيساهم فيروس ميركل في تكوّن ورم التوتة. في الآونة الأخيرة، كثفت الدراسات إلى أنه يوجد عو امل عديدة قد نزيد من خطورة سرطانات الرأس و الرقبة، ومن بينها على سبيل المثال التذخين و المشروبات الكحولية أو مسببات أخرى وراثية أو بيئية. بالإضـافة لهذه العوامل أثبتت الدر اسات أن التعرض لفيروس الورم الحليمي البشري (HPV) أو فيروس

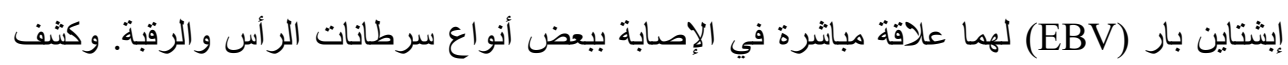
باحثون مؤخر اً عن وجود فيروس ميركل الورمي المتعدد (MCPyV) في عينات سرطانات اللسان 
الفصل الثالث من هذه الرسالة تم تقييم وجود الفيروسات الورمية المتعددة البشرية الجديدة في أنسجة سرطان القنوات الصفراوية من أجل التحقق من مساهنها المحتملة في تكوّن هذا الورم الخبيث. الهدف من هذات البحث هو استكثاف نسبة انتشار الفيروسات الورمية المتعددة على مستوى أنسجة وخلايا سرطان القتو ات

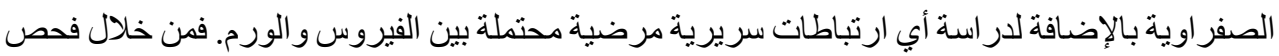

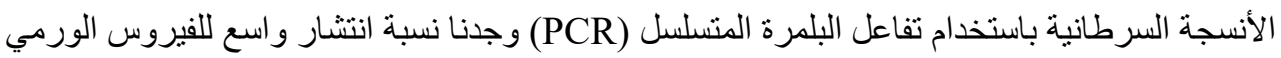

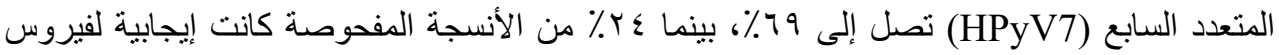
خلايا ميركل الورمي المتعدد (MCPyV)، وكان الفيروس الورمي المتعدد السادس (HPyV6) أقل

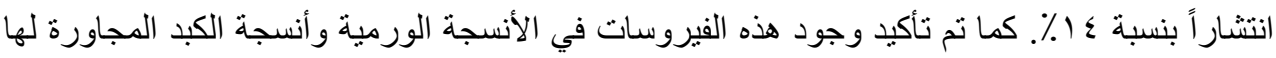

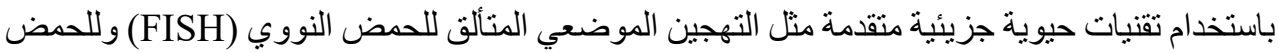
النووي الريبي (RISH) واستخدام كيمياء المناعة النسيجية لتحديد بروتين الفيروس (IHC). من النتائج

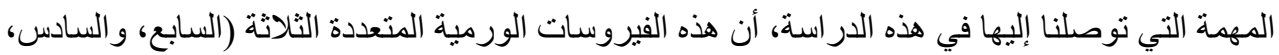
وميركل) تعتبر فيروسات كبدية أي قادرة على إصابة خلايا الكبد غير الورمية بالإضافة إلى إصابة خلايا

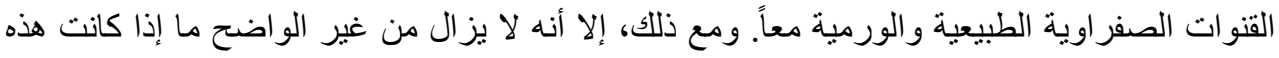

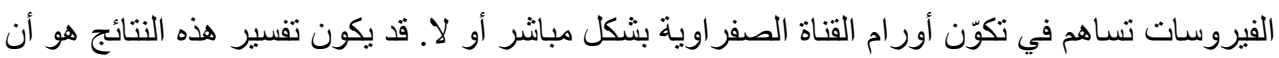
الاكتثاف المتكرر للفيروسات الورمية المتعددة خاصة الفيروس السابع (HPyV7) في الخلايا الكبدية الصفاقيّة المجاورة للقنوات الصفر اوية يشير إلى دور قوي غير مباشر لهذه الفيروسات من خلال التسبب

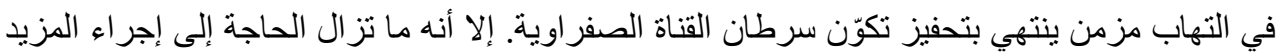
من الأبحاث للوصول إلى نتائج حاسمة.

يعتبر سرطان المثانة (Urothelial Cell Carcinoma) نوع شائع من الأور ام و غالبًا ما يبدأ سرطان

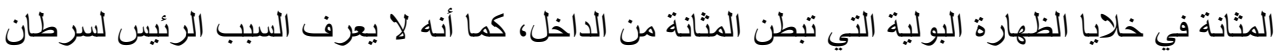

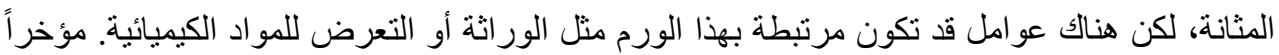

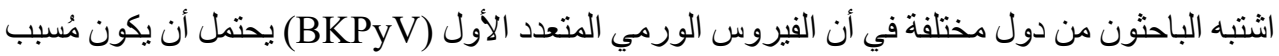

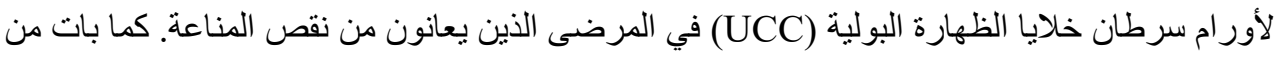
المعروف أن الفيروس الورمي المتعدد الأول (BKPyV) يكون كامناً في الخلايا الطلائية الأنبوبية الكلوية

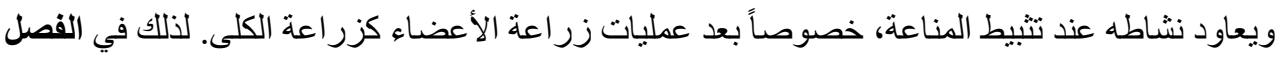
الرابع من الرسالة، قمنا بفصص الخلايا الطلائية المتواجدة في عينات بول من مرضى تم تتخيصهم

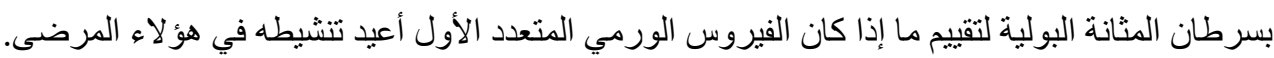

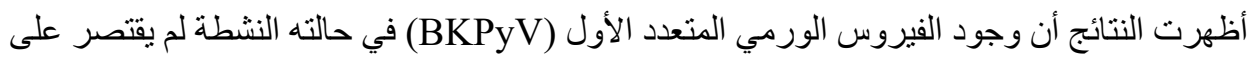


الرئيسي من الأبحاث في هذه الرسالة هو تسليط النظر على الآثار الخطيرة المحتملة عن تو اجد الفيروسات

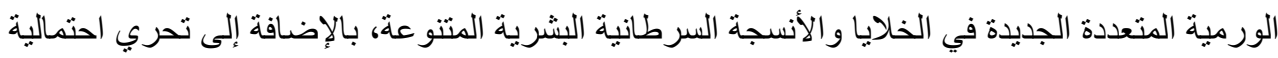

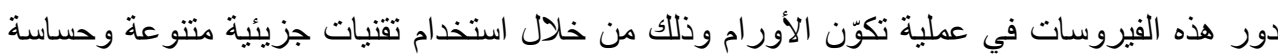
لفحص وجود هذا النوع من الفيروسات في الأنسجة و الخلايا السرطانية.

اشتصل الفصل الأول من هذه الرسالة على مقدمة عامة تلخص الدور المباثر أو غير المبانشر للفيروسات

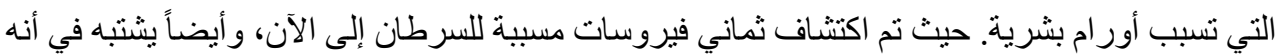

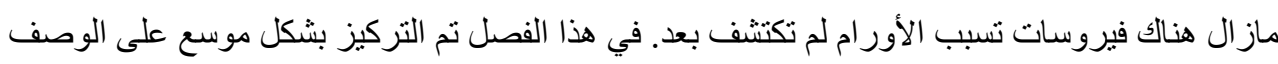

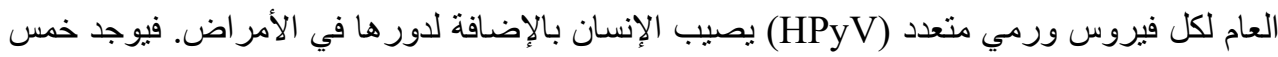

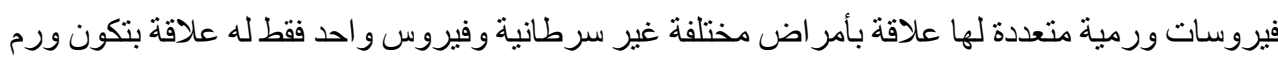
جلدي. في الفصل الثاني تضمن مر اجعة شاملة لما هو معروف حالياً حول ما إذا كان هناك دور لفيروسير فيرسين

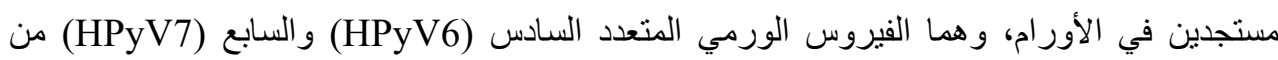

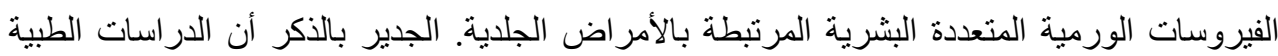

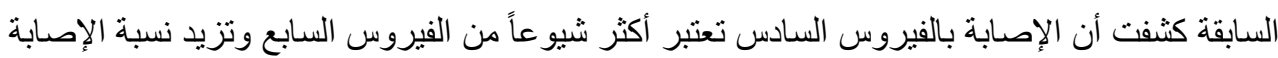

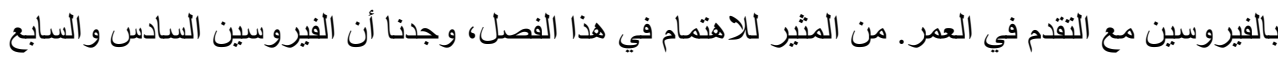

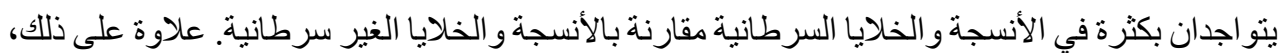

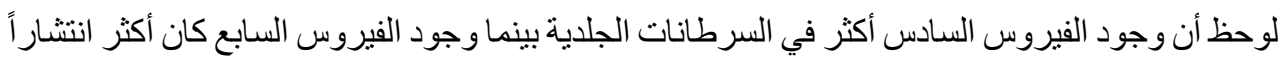
في سرطانات أخرى مثل سرطان القنوات الصفراوية وورم الغدة الصعترية. وبناءً على الدراسات التي لئي تمت مر اجعتها، خلص الفصل الثاني إلى احتمالية أن الفيروسين السادس والسابع من عائلة الفيروسات

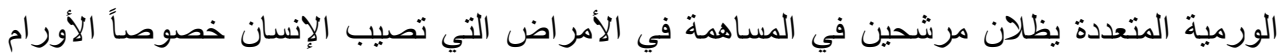
الجلاية.

يعتبر سرطان القنو ات الصفر اوية (CCA) ورم نادر وشديد الضراوة ويتشكل في الأنابيب التي تحمل

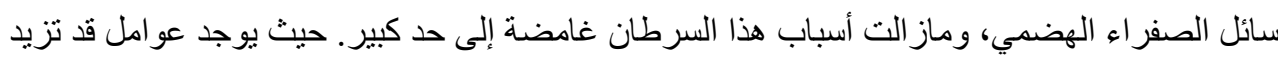

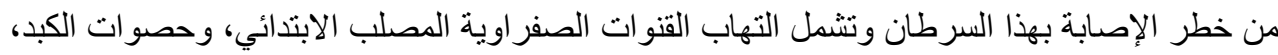

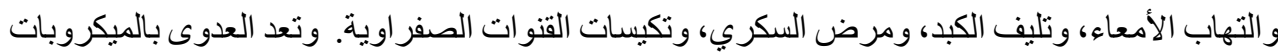

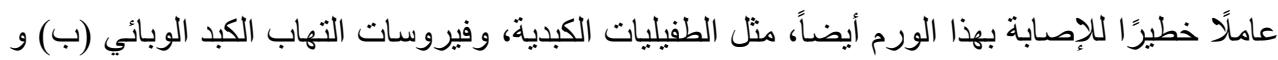

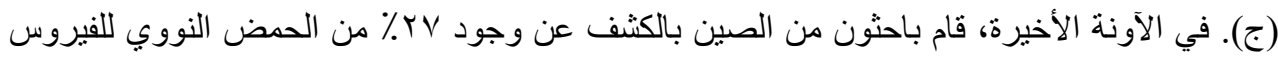

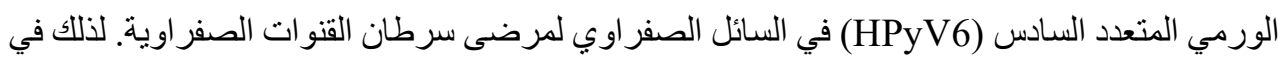




\section{الكثف عن السرطانات البشرية الحاملة للفيروسات الورمية المتعددة: آثثارها المرضيّة المترتبة على تكوّن الأورام}

\section{نبذة مختصرة عن الرسالة}

يعد السرطان من أحد الأسباب الرئيسة للوفيات في جميع أنحاء العالم ويعتبر مصدر قلق رئيسي للصحة

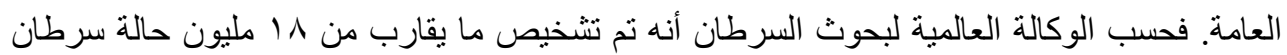

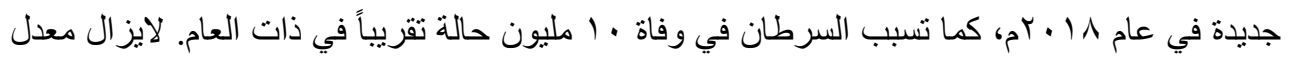

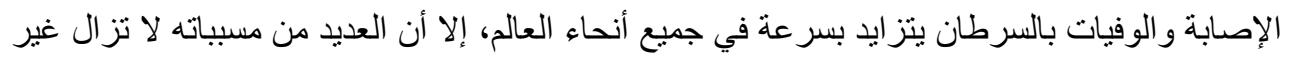

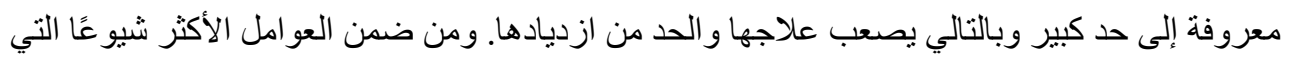

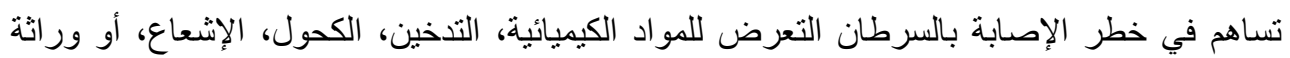

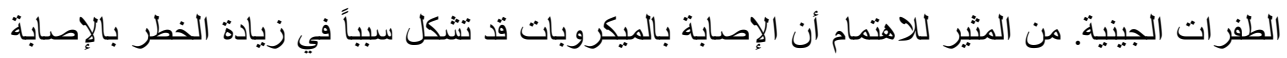

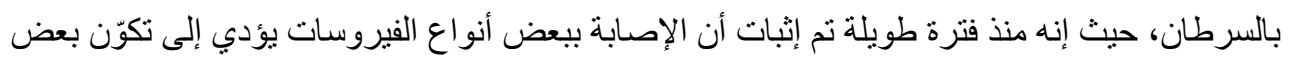

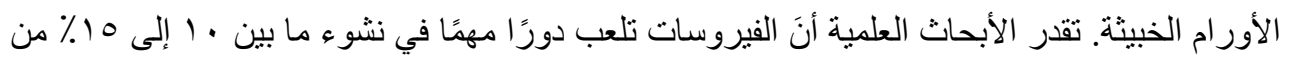

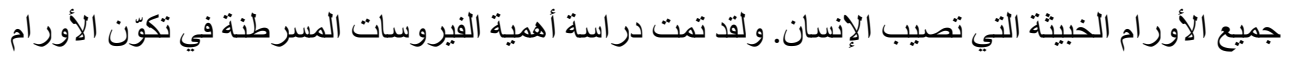
البشرية بشكل مكثف في العقد الماضي، مما ساهم في فهم مسببات بعض الأورام وتثخيصها و وعلاجها. لذلك اكتثاف ودراسة دور الفيروسات المعدية الناشئة التي يحتمل أن تسبب أو تساهم في تكوّن الأورام

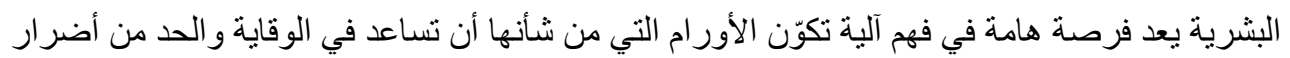
هذه الفيروسات بشكل فعال.

في هذه الرسالة، سيكون التركيز على الكثف عن الفيروسات الورمية المتعددة البشرية (HPyVs) في

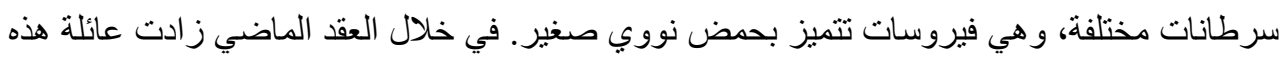
الفيروسات إلى أكثر من ץ • ا نوع قادرة على إصابة الثنييات و الطيور و الأسماك. خلال العقدين الأخيرين،

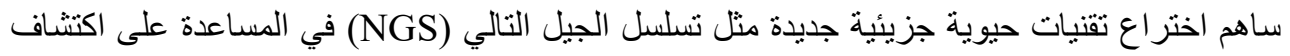
فيروسات جديدة، ومنها اكتثاف أعضاء جدد من عائلة الفيروسات الورمية المتعددة التي تصئب جلئ الإنسان (HPyVs)

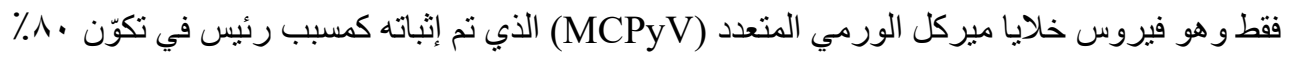

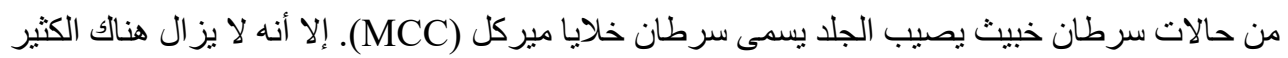

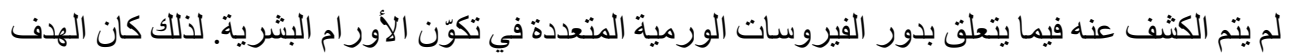




\section{شكر وتقدير}

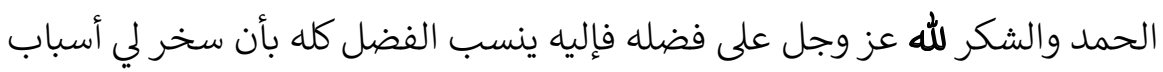

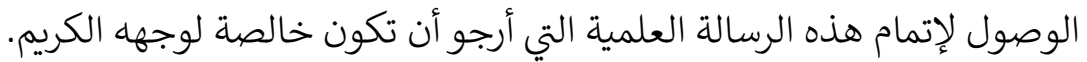

إلى من اشتاق إليها دوماً جدتي الحبيبة، لا تسعفني الكمات في شكرك على كرمك وحبك

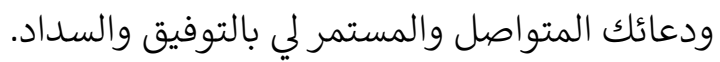

إلى قدوتي أبي وإلى أمي نبع المحبة والإيثار حفظكم الله، فمهما كتبت من عبارات الشكر

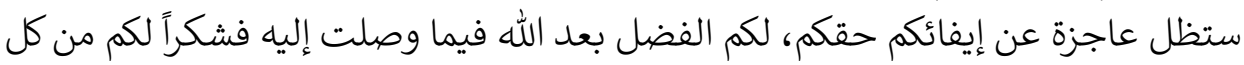
أعماق قلبي.

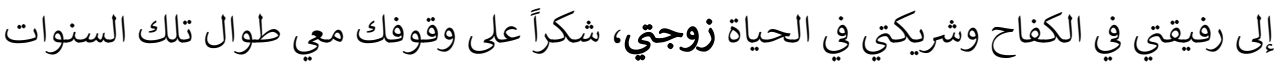

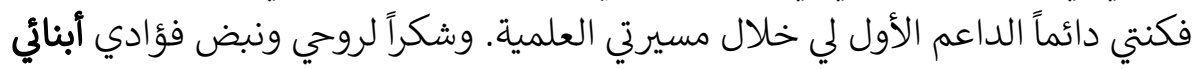

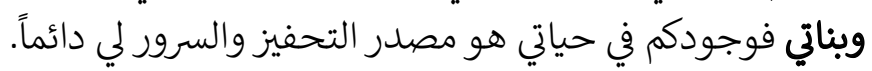

إلى إخواني وأخواتي، شكراً لكم فأنتم دائماً سندي وعضدي في الشدة والرخاء. وإلى الأقارب

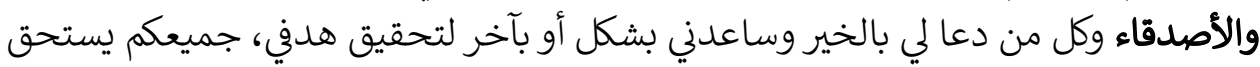
الشكر والثناء.

إلى وطني الغالي، لك عظيم الشكر والامتنان على الدعم السخي منذ بداية ابتعاثي

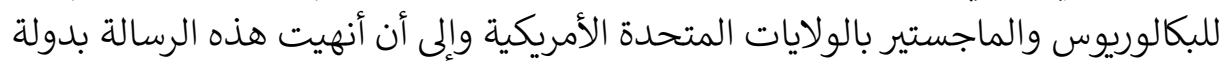
هولندا، مهما قدمت من كلمات الشكر لا أستطيع أن أوافيك حقدك الكيك.

كما أتوجه بالشكر لجامعة الباحة والملحقية الثقافية بدولة هولندا لكل ما قدموه لي من دعم واهتمام طوال فترة الابتعاث.

وأخيرًا، لم يكن بإمكاني إكمال هذه الرسالة بدون دعم المشرفين الدراسيين بالمركز الطبي

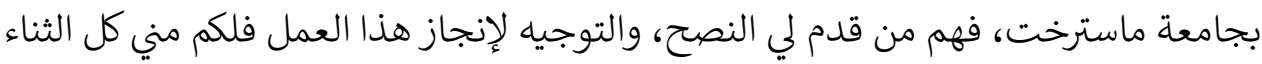
والتقدير.

\section{فيصل بن مهدي خلوفه}






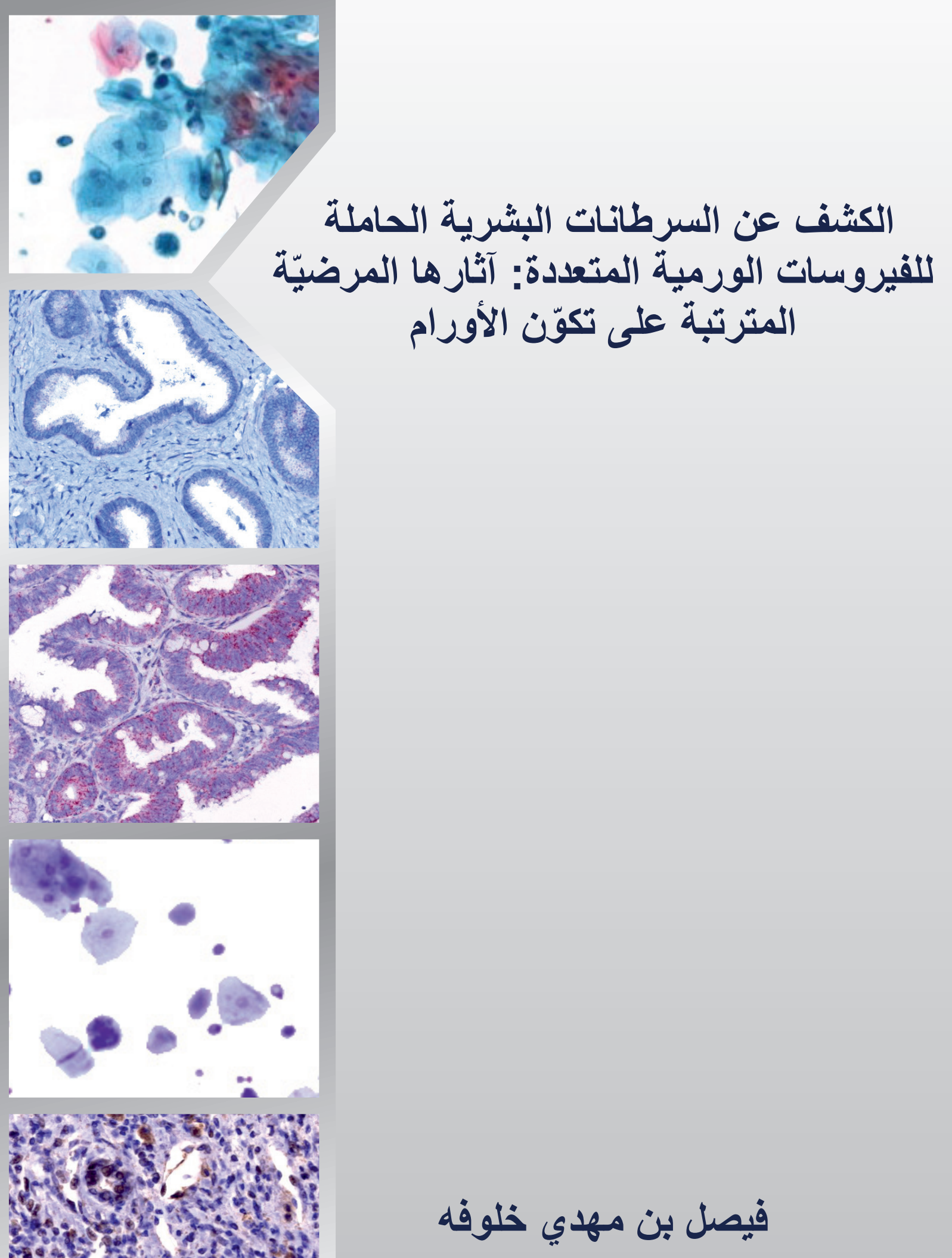

